\title{
EVOLUTIONARY AND ECOLOGICAL GENOMICS IN DEEP-SEA ORGANISMS
}

\author{
By \\ Santiago Herrera Monroy \\ B.Sc., Universidad de los Andes, Bogotá, Colombia, 2007 \\ M.Sc., Universidad de los Andes, Bogotá, Colombia, 2009 \\ Submitted in partial fulfillment of the requirements for the degree of \\ Doctor of Philosophy \\ at the \\ MASSACHUSETTS INSTITUTE OF TECHNOLOGY \\ and the \\ WOODS HOLE OCEANOGRAPHIC INSTITUTION
}

February 2015

(C) 2015 Santiago Herrera Monroy

All rights reserved.

The author hereby grants to MIT and WHOI permission to reproduce and to distribute publicly paper and electronic copies of this thesis document in whole or in part in any medium now known or hereafter created.

Signature of Author

Joint Program in Oceanography/Applied Ocean Science and Engineering

Massachusetts Institute of Technology and Woods Hole Oceanographic Institution

December 30, 2014

Certified by

Dr. Timothy M. Shank

Thesis Supervisor

Accepted by

Professor Martin F. Polz

Chair, Joint Committee for Biological Oceanography

Massachusetts Institute of Technology and Woods Hole Oceanographic Institution 


\title{
EVOLUTIONARY AND ECOLOGICAL GENOMICS IN DEEP-SEA ORGANISMS
}

\author{
By Santiago Herrera Monroy \\ Submitted to the MIT/WHOI Joint Program in Oceanography/Applied Ocean Science and Engineering \\ on December 30, 2014 in partial fulfillment of the requirements for the degree of \\ Doctor of Philosophy in Biological Oceanography
}

\begin{abstract}
Hydrothermal vents and coral ecosystems are conspicuous biological hot spots in the deep-sea. These ecosystems face increasing threats from human activities. Having thorough taxonomic inventories as well as understanding species' relatedness, genetic diversity, connectivity patterns, and adaptive potential is fundamental for the implementation of conservation strategies that help mitigate these threats. This thesis provides fundamental high-priority knowledge in taxonomic, evolutionary, and ecological aspects of deep-sea coral and vent species, by harnessing the power of genomic tools and overcoming long-standing methodological barriers. First, I develop bioinformatic tools that help guide the design of studies aiming to characterize eukaryotic genome diversity using restriction-site associated DNA sequencing. Using these tools I find that the predictability of restriction site frequencies in eukaryotic genomes is chiefly determined by the phylogenetic position of the target species and the recognition sequence of the selected restriction enzyme. These tools are then applied to test global-scale historical biogeographic hypotheses of vent fauna using barnacles as model. Phylogeographic inferences suggest that the western Pacific was the place of origin of the major vent barnacle lineage, followed by circumglobal colonization eastward along the southern hemisphere during the Neogene. I suggest that the geological processes and dispersal mechanisms discussed here can explain distribution patterns of many other marine taxa in addition to barnacles. Regional-scale analyses indicate that vent barnacle populations are well connected within basins and ridge systems, and that their diversity patterns do not conform to the predictions from the hypothesis that seamounts are centers of endemism. I then move on to resolve long-standing questions regarding species definitions in recalcitrant deep-sea coral taxa, by unambiguously resolving evolutionary relationships and objectively inferring species boundaries. Finally, I explore the adaptive potential of deep-sea coral species to environmental changes by examining a case of adaptation to shallow water from the deep sea. Candidate positive-selection markers shared between pairs of shallow and deep populations are identified as likely makers for genomic regions involved in adaptation. Overall, the results from this thesis constitute critical baseline data with which to assess potential effects of anthropogenic disturbances on deep-sea ecosystems.
\end{abstract}

Thesis supervisor:

Dr. Timothy M. Shank

Title: Associate Scientist with Tenure, Biology Department, Woods Hole Oceanographic Institution 
Para mis papás, Liliana y Mauricio 


\section{AKNOWLEDGEMENTS}

This degree is the fulfillment of a lifelong dream. I am infinitely grateful to Tim Shank, for taking a chance with me, for challenging me constantly, for honest advising, for providing limitless opportunities to pursue my interests and develop my skills, for unconditional support and trust, and for making me feel more like a colleague than a student since the first day. I could not imagine a better place to have done my $\mathrm{PhD}$. I look forward to many more ventures to come.

I am extremely grateful to my committee Ann Tarrant, Adam Reitzel, and Martin Polz for guiding me along the way and being my best advocates. Ann, thank you for always having an open door, for being ready to help at a minute's notice, and for being generous sharing your knowledge and giving thorough feedback. Adam, thank you for your contagious enthusiasm for science and inspiring creativity; I greatly enjoy working with you. I am also grateful for Mark Hahn for chairing my defense and offering excellent advise.

To Juan Sánchez, Steve Cairns, and Allen Collins, thank you for your mentorship and support, which fostered my passion for science and lead me to pursue this degree.

Special thanks to the Academic Programs Office at WHOI, the MIT-WHOI Joint Program Office, the MIT/WHOI Joint Program Committee, the Joint Committee for Biological Oceanography, the administrative staff at both WHOI and MIT, and the support, maintenance, security and facilities staff at WHOI for your dedication and hard work behind the scenes. I am also thankful to all the course instructors and teaching assistance for sharing their knowledge and fostering critical thinking.

To all my friends in the lab and around Woods Hole, particularly Ellie Bors, Arthur Olive, Cat Munro, Larry George, Jason Kapit, Kalina Gospodinova, Amalia Aruda, Albert Almada, Kristen Hunter-Cevera, Kerry McCulloch, Taylor Heyl, Annette Govindarajan, Walter Cho, Breea Govenar, Kate Buckman, Taylor Crockford, Liz Drenkard and Britta Voss, thank you for making this such an enjoyable time.

Gracias a toda mi familia por su amor y apoyo, pero en especial a mis padres Mauricio y Liliana. Papás, todos mis logros se los debo a ustedes. Gracias por su incondicionalidad, sacrificio, y ejemplo.

To Jill, thank you for being my loyal partner through this journey, and I look forward to being by your side in all the journeys to come.

I thank P. Aldersdale (CSIRO), N. Ardila (ECOMAR) and J. Sanchez (U. Andes) for assistance with morphological specimen identifications. I also thank my outstanding diving team E. O'Brien (WHOI), D. Forsman (WHOI), J. Fellows (WHOI), and Canadian collaborators V. Tunnicliffe (U. Victoria), J. \& S. Schooner, K. Heylar, and N. McDaniel for invaluable assistance during scuba diving fieldwork in British Columbia (DFO scientific license FIN130270). The times spent underwater are some of my favorite memories.

This research was supported by the Office of Ocean Exploration and Research of the National Oceanic and Atmospheric Administration (NA09OAR4320129 to TMS); the Division of Ocean Sciences of the National Science Foundation (OCE-1131620 to TMS); the Division of Polar Programs of the National Science Foundation (PLR-0739675 to TMS); the Astrobiology Science and Technology for Exploring Planets program of the National Aeronautics and Space Administration (NNX09AB76G to TMS); the National Geographic Society/Waitt Foundation (W285-13 to SH); and the Academic Programs Office (Ocean Ventures Fund and fellowship support to $\mathrm{SH}$ ), the Ocean Exploration Institute (fellowship support 
to TMS and $\mathrm{SH}$ ) and the Ocean Life Institute (internal grant to TMS and SH) of the Woods Hole Oceanographic Institution.

For enabling access to key specimens I thank S. Mills (NIWA), K. Schnabel (NIWA), D. Tracey (NIWA), M. Clark (NIWA), A. Rowden (NIWA), K. Iizasa (U. Tokyo), Y. Suzuki (U. Tokyo), S. Nakagawa (JAMSTEC), P. Tyler (NOCS), J. Copley (NOCS), A. Rogers (Oxford), N. Roterman (Oxford), J. Resing (NOAA-PMEL), R. Embley (NOAA-PMEL), A. Reysenbach (PSU), M.K. Tivey (WHOI), P. Fryer (UH), C. Langmuir (Harvard), K. Von Damm (UNH), M. Lilley (UW), S. Cairns (Smithsonian), E. Cordes (Temple U.), A. Quattrini (Temple U.), G. Workman (DFO), M. Wyeth (DFO), K. Anderson (DFO), M. Frey (RBCM), H. Gartner (RBCM), Ed Bowlby (NOAA), E. Edinger (Memorial U.), J. Sanchez (U. Andes), L. Watling (U. Hawaii), J. Adkins (CalTech) the masters, crew, scientific personnel, and funding agencies of expeditions AT03-28, AT07-06, AT07-35, JC042, JC067, KM0417, KM0912, KOK0505, KOK0506, Lophelia II 2009, NT97-10, NT97-14, NT99-09, RB-0503, RR1211, TAN1007, TAN1104, TAN1206, TAN1213, TN234, TN236, YK06-13, and YK09-13.

Specimens provided by the National Institute of Water and Atmospheric Research (NIWA) were collected under research programs: Kermadec Arc Minerals, funded by the New Zealand Ministry of Business, Innovation \& Employment (MBIE), Auckland University, Institute of Geological and Nuclear Science (GNS), and WHOI; Ocean Survey 20/20 funded by Land Information New Zealand; Impact of resource use on vulnerable deep-sea communities (CO1X0906), funded by MBIE; Nascent Inter-Ridge Volcanic And Neotectonic Activity, funded by the Ministry for Primary Industries (MPI), GNS, MBIE, and the U. of New Hampshire; Scientific Observer Program funded by MPI; and the Joint New ZealandUSA 2005 NOAA Ring of Fire Expedition, part of NIWA's Seamount Program (FRST CO1X0508). 


\section{CONTENTS}

$\begin{array}{lr}\text { ABSTRACT } & 3\end{array}$

$\begin{array}{ll}\text { ACKNOWLEDGEMENTS } & 7\end{array}$

$\begin{array}{lr}\text { CONTENTS } & 9\end{array}$

CHAPTER 1: Introduction 11

CHAPTER 2: Genome-wide predictability of restriction sites across the eukaryotic tree of life

CHAPTER 3: Evolutionary and biogeographical patterns of barnacles from deep-sea hydrothermal vents

CHAPTER 4: No evidence of seamount-driven isolation in deep-sea hydrothermal vent barnacle populations

CHAPTER 5: RAD sequencing enables unprecedented phylogenetic resolution and objective species delimitation in recalcitrant divergent taxa

CHAPTER 6: The genomics of adaptation potential of deep-sea corals to environmental changes 


\section{CHAPTER 1}

\section{Introduction}

\section{Threats to deep-sea coral ecosystems}

Deep-sea corals are some of the most conspicuous invertebrate inhabitants of hard-bottom deep-sea benthic environments worldwide. They are not only more diverse in terms of number of species than their shallow counterparts (Cairns 2007), but they also play a fundamental role as foundation species and ecosystem engineers, creating three-dimensional habitats that are occupied by a high diversity of associate species (Buhl-Mortensen \& Mortensen 2005; Costello et al. 2005; Buhl-Mortensen et al. 2010; Watling et al. 2011). Deep-sea ecosystems also support fisheries (D'Onghia et al. 2011) and have been identified as important sources of marine natural products (Leal et al. 2012). Deep-sea corals, generally speaking, have evolved in a relatively stable and energy-poor environment. They are characterized by slow growth rates, extreme longevity, low fecundity, and a late age of maturity (Roberts et al. 2009). These characteristics make deep-sea coral ecosystems fragile and with low resilience to the severe disturbances generated by many modern human activities, including physical damage caused by bottom-trawling fishing (Watling \& Norse 1998; Koslow et al. 2001; Waller et al. 2007; Althaus et al. 2009; Clark \& Rowden 2009; Williams et al. 2010), climate change and ocean acidification caused by emissions of greenhouse gasses (Doney et al. 2009), pollution and habitat destruction generated by waste disposal (Kvassnes et al. 2012), deep-sea mining (Van Dover 2010), and off-shore drilling for hydrocarbons (White et al. 2012). As such, the United Nations has designated deep-sea coral ecosystems as Vulnerable Marine Ecosystems (http://www.un.org/depts/los/general_assembly/general_assembly_resolutions.htm UN General Assembly resolutions 61/105 and 64/72), requiring new management and protection strategies, such as the U.S. Magnuson-Stevens Fishery Conservation and Management Reauthorization Act of 2006. The MagnusonStevens act authorized Regional Fishery Management Councils to designate zones to protect deep-sea corals from damage caused by fishing gear, and established a Deep-Sea Coral Research and Technology Program to advance a critical understanding of their taxonomy and systematics, biogeography, genetic connectivity, and physiological responses to stressors in deep water coral ecosystems.

\section{Threats to hydrothermal vent ecosystems}

Benthic chemosynthetic ecosystems present a sharp contrast to other ecosystems in the deep-sea. They are characterized by high rates of in situ primary productivity, marked patchiness, and highly dynamic geological settings (Van Dover 2000). Deep-sea hydrothermal vent environments can have extremely 
steep gradients of chemistry and temperature, and high disturbance frequencies given their occurrence on volcanic or actively spreading tectonic systems. As a consequence, hydrothermal vent environments present extreme selective pressures on evolutionary timescales, and can yield vent ecosystems with relatively low biodiversity and high endemism (Van Dover 2010). These very characteristics may make them susceptible to disturbances caused by mining of polymetallic sulfides. Although organisms at deepsea hydrothermal vents have adapted to cope with natural disturbances, the frequency and magnitude at which these occur can vary greatly depending on the particular geophysical nature of each system (Baker $\&$ German 2004). Thus, disturbance from mining could have additive effects to natural disturbances at scales not previously experienced by these organisms, which could potentially lead to significant losses of biodiversity. There is a surging need to provide the highest-priority information needed to design optimal conservation and management strategies for areas that are being prospected for mining (Van Dover et al. 2012). These priorities emphasize the identification of conservation units at the genetic, species and biogeographic levels, and a better understanding of connectivity among populations.

\section{Priorities for conservation of deep-sea ecosystems}

Knowledge of conservation units is fundamental for the creation and implementation of efficient strategies that help mitigate the effects of harmful human activities on deep-sea ecosystems. Such knowledge must include well-founded taxonomic inventories that allow us to identify species and ecosystems at risk, as well as an understanding of their relatedness, genetic variance, distribution, connectivity patterns, and adaptation potential (Christensen et al. 1996; Dubois 2003; Roberts \& Cairns 2014). Nevertheless, gaining this knowledge in deep-sea ecosystems is difficult due to the extreme challenges of working in these environments, combined with the paucity of genetic resources for deep-sea taxa.

\section{Issues of traditional phylogenetic approaches}

Traditionally, phylogenetic and population genetic studies in non-model organisms - which aims include understanding species boundaries, relationship patterns, evolutionary histories, factors that diminish or promote genetic diversity, demographic processes of populations, and interactions with environmental conditions - have based their power on a handful of homologous DNA sequence markers. Target DNA sequence markers can be easily sequenced using nearly-universal primers; however, several problems have been identified with the use of the few traditional sequence markers available for non-model organisms (e.g., mitochondrial and ribosomal genes), including low variability, biased loci sampling, poor genome representation and small statistical power, presence of pseudogenes, multiple gene copies, and non-independence caused by linkage (Brumfield 2003; Brito \& Edwards 2008). Microsatellites emerged 
during the last decade as a novel class of markers promising great potential to solve population-level questions. However, many problems have identified with the use of microsatellites, including poor understanding of their mutational processes, high rates of back mutations and homoplasy, presence of null alleles, low reproducibility and comparability of results, and high monetary and time expenses for individual marker development and genotyping (Brumfield 2003).

\section{Opportunities of novel genomic approaches}

The problems related to the use of traditional genetic markers have been recognized and accounted for in model organisms by comparing large amounts of genomic sequence information among individuals and identifying thousands of variable regions, such as single nucleotide polymorphisms (SNPs) across the genome, e.g. Clark et al. (2007) and Rokas et al. (2003). Single nucleotide polymorphisms (SNPs) have been recognized as the most prevalent source of variability in any given genome; they represent $c a$. 90\% of the genetic variation in the human genome (Collins et al. 1999). As such, SNPs overcome most of the problems related with the use of traditional sequence-markers - they are present in extremely high numbers across the genome, have a wide range of mutational rates, behave largely as independent loci, and can be screened with high-throughput techniques, making them economical (Brumfield 2003). Technological and methodological developments in next-generation sequencing platforms over the last five years (e.g., Illumina, PacBio, IonTorrent, etc) have made genomic resources for SNP development and genotyping increasingly accessible and available to researchers investigating a wide spectrum of evolutionary questions in diverse organisms. Their use is now being successfully implemented in several non-model organisms, thus offering a great opportunity to overcome the difficulties inherent to the use of traditional approaches in many taxa.

SNPs have been successfully used to resolve evolutionary and biogeographic histories of diverse taxa, from bees (Whitfield et al. 2006), to humans (Jakobsson et al. 2008), nematodes (Andersen et al. 2012), anemones (Reitzel et al. 2013), and pitcher plant mosquitoes (Emerson et al. 2010). Recently developed methodologies allow the implementation of next-generation sequencing technologies for the rapid detection and genotyping of SNPs in organisms without reference genomes (Garvin et al. 2010); the socalled genotyping-by-sequencing (GBS) approaches (Baird et al. 2008). Restriction-site associated DNA sequencing (RAD-seq), a kind of GBS approach, enables high-throughput sequencing of homologous sites in nuclear genome after a complexity-reduction step carried out with a high-fidelity restriction enzyme. This revolutionary approach has opened a new frontier in molecular studies, with the promise of providing profound insights into the genetics, organismal biology, ecology and evolution of wild populations (Seeb et al. 2011). 


\section{Objective and outline}

With this thesis, I aim to provide fundamental high-priority knowledge in taxonomic, genetic, evolutionary, and ecological aspects of deep-sea coral and hydrothermal vent species, by harnessing the power of novel genomic tools. This knowledge could be applied to the conservation and management of deep-sea ecosystems and their biodiversity.

To achieve this goal, I have performed bioinformatic and empirical studies implementing restriction-site associated DNA sequencing methodologies on ecologically important deep-sea coral and hydrothermal vent species. I present results from my investigations on the causes and consequences of evolutionary forces that determine biodiversity patterns in the deep-sea. In Chapter 2, I test the hypothesis that genome composition can be used to predict the number of restriction sites for a given combination of restriction enzyme and genome across the eukaryotic tree of life. I develop a methodology to predict the frequency of restriction sites that helps guide the design of GBS studies in eukaryotic organisms. This methodology is then used throughout the rest of the thesis. Chapters 3-6 can be categorized into chapters that examine macroevolutionary processes above the species level (chapters 3 and 5), and chapters that examine microevolutionary processes within species (chapters 4 and 6). Alternatively, they can also be categorized according to the target taxon system: deep-sea hydrothermal vent barnacles (chapters 3 and 4), or deepsea corals (chapters 5 and 6) (Fig. 1).

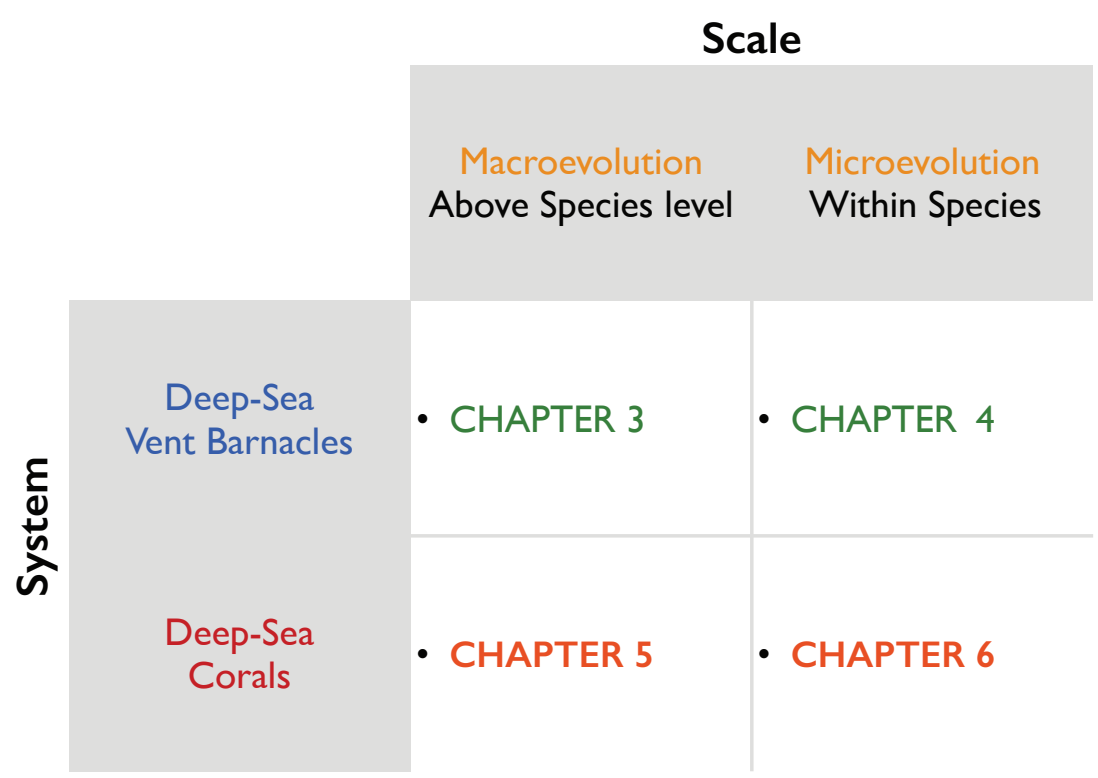

Figure 1. Conceptual categorization of chapters according to the time scale of the processes and the target taxon systems examined. 
In Chapter 3, I compare traditional DNA sequence makers with novel genomic data from restriction site associated DNA sequencing (RAD-seq) to characterize the global genetic diversity of barnacles from deep-sea hydrothermal vents, which due to their widespread but discontinuous distribution represent an excellent model for testing global biogeographic hypotheses. I infer their time and place of origin, mode of dispersal, and diversification throughout the world's vents. I then complement this research in Chapter 4 , by using vent barnacles as a model to test smaller-scale biodiversity hypothesis. Specifically, I test the hypothesis that seamounts act as islands promoting divergence and speciation in deep-sea vent fauna. For this I compare the genetic diversity contained in single nucleotide polymorphisms (SNPs) obtained through RAD-seq to examine population-structuring patterns of populations in different barnacle species from seamount and spreading ridges.

In Chapter 5, I demonstrate the empirical utility of RAD-seq to solve evolutionary questions in deeper time by unambiguously resolving phylogenetic relationships among recalcitrant octocoral taxa with divergences greater than 80 million years and performing unambiguous species delimitations. In Chapter 6, I make use of the knowledge gained in defining species boundaries in deep-sea coral species by addressing questions regarding interactions of coral populations from the same species with their environment, in shorter time scales. I focus on a deep-sea coral species that can also be found in shallow high-latitude fjords with distinct environmental conditions from those found in the deep-sea. Here I aim to identify and characterize genomic regions that have may have enabled the successful adaptation to shallow-water in this deep-sea species. Finally, I summarize findings and draw general conclusions in Chapter 7.

\section{REFERENCES}

Althaus F, Williams A, Schlacher T, et al. (2009) Impacts of bottom trawling on deep-coral ecosystems of seamounts are long-lasting. Marine Ecology Progress Series 397, 279-294.

Andersen EC, Gerke JP, Shapiro JA, et al. (2012) Chromosome-scale selective sweeps shape Caenorhabditis elegans genomic diversity. Nature Genetics 44, 285-U283.

Baird NA, Etter PD, Atwood TS, et al. (2008) Rapid SNP discovery and genetic mapping using sequenced RAD markers. PLoS One 3, e3376.

Baker ET, German CR (2004) On the global distribution of hydrothermal vent fields. In: Mid-Ocean Ridges, pp. 245-266. American Geophysical Union.

Brito PH, Edwards SV (2008) Multilocus phylogeography and phylogenetics using sequence-based markers. Genetica 135, 439-455.

Brumfield R (2003) The utility of single nucleotide polymorphisms in inferences of population history. Trends in Ecology \& Evolution 18, 249-256.

Buhl-Mortensen L, Mortensen PB (2005) Distribution and diversity of species associated with deep-sea gorgonian corals off Atlantic Canada. In: Cold-Water Corals and Ecosystems (eds. Freiwald AR, Roberts JM), pp. 849-879. Springer-Verlag, Berlin Heidelberg. 
Buhl-Mortensen L, Vanreusel A, Gooday AJ, et al. (2010) Biological structures as a source of habitat heterogeneity and biodiversity on the deep ocean margins. Marine Ecology 31, 21-50.

Cairns S (2007) Deep-water corals: an overview with special reference to diversity and distribution of deep-water scleractinian corals. Bulletin of Marine Science 81, 311-322.

Christensen NL, Bartuska AM, Brown JH, et al. (1996) The report of the Ecological Society of America committee on the scientific basis for ecosystem management. Ecological Applications 6, 665-691.

Clark AG, Eisen MB, Smith DR, et al. (2007) Evolution of genes and genomes on the Drosophila phylogeny. Nature 450, 203-218.

Clark MR, Rowden AA (2009) Effect of deepwater trawling on the macro-invertebrate assemblages of seamounts on the Chatham Rise, New Zealand. Deep-Sea Research Part I 56, 1540-1554.

Collins FS, Brooks LD, Chakravarti A (1999) A DNA polymorphism discovery resource for research on human genetic variation (vol 8, pg 1229, 1998). Genome Research 9, 210-210.

Costello M, McCrea M, Freiwald A, et al. (2005) Role of cold-water Lophelia pertusa coral reefs as fish habitat in the NE Atlantic. In: Cold-Water Corals and Ecosystems (eds. Freiwald A, Roberts JM), pp. 771-805. Springer Berlin Heidelberg.

D'Onghia G, Indennidate A, Giove A, et al. (2011) Distribution and behaviour of deep-sea benthopelagic fauna observed using towed cameras in the Santa Maria di Leuca cold-water coral province. Marine Ecology Progress Series 443, 95-110.

Doney SC, Balch WM, Fabry VJ, Feely RA (2009) Ocean acidification: A critical emerging problem for the ocean sciences. Oceanography 22, 16-25.

Dubois A (2003) The relationships between taxonomy and conservation biology in the century of extinctions. Comptes Rendus Biologies 326, S9-S21.

Emerson KJ, Merz CR, Catchen JM, et al. (2010) Resolving postglacial phylogeography using highthroughput sequencing. Proceedings of the National Academy of Sciences of the United States of America 107, 16196-16200.

Garvin MR, Saitoh K, Gharrett AJ (2010) Application of single nucleotide polymorphisms to non-model species: a technical review. Molecular Ecology Resources 10, 915-934.

Jakobsson M, Scholz SW, Scheet P, et al. (2008) Genotype, haplotype and copy-number variation in worldwide human populations. Nature 451, 998-1003.

Koslow JA, Gowlett-Holmes K, Lowry JK, et al. (2001) Seamount benthic macrofauna off southern Tasmania: community structure and impacts of trawling. Marine Ecology Progress Series 213, 111-125.

Kvassnes AJ, Sweetman AK, Hobæk A, Thorseth IH, Bolam S (2012) Imptail - Improved submarine tailing placements (STPS) in Norwegian Fjords. In: Ocean Sciences Meeting. Abstract ID:11074, Salt Lake City, Utah.

Leal MC, Puga J, Serodio J, Gomes NCM, Calado R (2012) Trends in the discovery of new marine natural products from invertebrates over the last two decades - where and what are ee bioprospecting? PLoS One 7, e30580.

Reitzel AM, Herrera S, Layden MJ, Martindale MQ, Shank TM (2013) Going where traditional markers have not gone before: utility of and promise for RAD sequencing in marine invertebrate phylogeography and population genomics. Molecular Ecology 22, 2953-2970.

Roberts JM, Cairns SD (2014) Cold-water corals in a changing ocean. Current Opinion in Environmental Sustainability 7, 118-126.

Roberts JM, Wheeler A, Freiwald AR, Cairns SD (2009) Cold-Water Corals : The Biology and Geology of Deep-Sea Coral Habitats Cambridge University Press, Cambridge, UK ; New York.

Rokas A, Williams BL, King N, Carroll SB (2003) Genome-scale approaches to resolving incongruence in molecular phylogenies. Nature 425, 798-804.

Seeb LW, Templin WD, Sato S, et al. (2011) Single nucleotide polymorphisms across a species' range: implications for conservation studies of Pacific salmon. Molecular Ecology Resources 11, 195217. 
Van Dover CL (2000) The Ecology of Deep-Sea Hydrothermal Vents Princeton University Press, Princeton, N.J.

Van Dover CL (2010) Mining seafloor massive sulphides and biodiversity: what is at risk? ICES Journal of Marine Science 68, 341-348.

Van Dover CL, Smith CR, Ardron J, et al. (2012) Designating networks of chemosynthetic ecosystem reserves in the deep sea. Marine Policy 36, 378-381.

Waller R, Watling L, Auster P, Shank T (2007) Anthropogenic impacts on the Corner Rise seamounts north-west Atlantic Ocean. Journal of the Marine Biological Association of the United Kingdom 87, 1075-1076.

Watling L, France SC, Pante E, Simpson A (2011) Biology of deep-water octocorals. Advances in Marine Biology 60, 41-122.

Watling L, Norse EA (1998) Disturbance of the seabed by Mobile Fishing Gear: A Comparison to Forest Clearcutting. Conservation Biology 12, 1180-1197.

White HK, Hsing P-Y, Cho W, et al. (2012) Impact of the Deepwater Horizon oil spill on a deep-water coral community in the Gulf of Mexico. Proceedings of the National Academy of Sciences of the United States of America 109, 20303-20308.

Whitfield CW, Behura SK, Berlocher SH, et al. (2006) Thrice out of africa: Ancient and recent expansions of the honey bee, Apis mellifera. Science 314, 642-645.

Williams A, Schlacher TA, Rowden AA, et al. (2010) Seamount megabenthic assemblages fail to recover from trawling impacts. Marine Ecology 31, 183-199. 


\title{
CHAPTER 2
}

\section{Genome-wide predictability of restriction sites across the eukaryotic tree of life}

\author{
Santiago Herrera ${ }^{1,2}$ Paula H. Reyes-Herrera ${ }^{3}$, Timothy M. Shank ${ }^{1}$ \\ ${ }^{1}$ Biology Department, Woods Hole Oceanographic Institution, 266 Woods Hole Road, Woods Hole, MA \\ 02543, USA \\ 2 MIT/WHOI Joint Program, Massachusetts Institute of Technology, 77 Massachusetts Avenue, \\ Cambridge, MA 02139, USA \\ ${ }^{3}$ Facultad Ingeniería de Electrónica y Biomédica, Universidad Antonio Nariño, Carrera 3 Este \# 47a -15, \\ Bloque 4, Bogotá, Colombia.
}

Preprint available online: Herrera S., P.H. Reyes-Herrera \& T.M. Shank (2014) Genome-wide predictability of restriction sites across the eukaryotic tree of life. bioRxiv, doi: http://dx.doi.org/10.1101/007781. Git code repository: https://github.com/phrh/PredRAD

\begin{abstract}
High-throughput sequencing of reduced representation libraries obtained through digestion with restriction enzymes - generally known as restriction site associated DNA sequencing (RAD-seq) - has become a common strategy to generate genome-wide genotypic and sequence data from eukaryotes. The choice of restriction enzyme is critical for the design of any RAD-seq study as it determines the number of genetic markers that can be obtained for a given taxon enabling a broad spectrum of applications, including marker discovery, population genomics, genomic mapping and phylogenetics. Here, we test the hypothesis that genome composition, in terms of GC content, and mono-, di- and trinucleotide composition, can be used to predict the number of restriction sites for a given combination of restriction enzyme and genome across the eukaryotic tree of life. Our analyses reveal that in most cases the trinucleotide genome composition model is the best predictor of the expected number of restriction sites in a eukaryotic genome, and the GC content and mononucleotide models the worst. We conclude that the predictability of restriction site frequencies in eukaryotic genomes needs to be treated on a case-specific
\end{abstract}


basis, whereby the phylogenetic position of the taxon of interest and the specific recognition sequence of the selected restriction enzyme are the chief foci among the most determinant factors. The software here developed, PredRAD (https://github.com/phrh/PredRAD), and the resulting databases constitute a valuable reference resource that will help guide the choice of restriction enzyme for any study using RAD-seq or related methods.

\section{INTRODUCTION}

The use of restriction enzymes to obtain reduced representation libraries from nuclear genomes, combined with the power of next-generation sequencing technologies, is rapidly becoming one of the most used strategies to generate genome-wide genotypic and sequence data in both model and non-model organisms (Baird et al. 2008; Andolfatto et al. 2011; Elshire et al. 2011; Peterson et al. 2012). The hundreds, thousands or tens of thousands of single nucleotide polymorphisms (SNPs) embedded in the resulting restriction site associated DNA (RAD) sequence tags (Miller et al. 2007; Baird et al. 2008) have a myriad of uses in biology ranging from genetic mapping (Wang et al. 2013; Weber et al. 2013) to population genomics (Hohenlohe et al. 2010; Andersen et al. 2012; White et al. 2013), phylogeography (Emerson et al. 2010; Reitzel et al. 2013), phylogenetics (Wagner et al. 2012; Eaton \& Ree 2013), and SNP marker discovery (Scaglione et al. 2012; Toonen et al. 2013).

The choice of appropriate restriction enzyme(s) is critical for the effective design and application of RAD sequencing and a rapidly growing number of related methods such as genotyping-by-sequencing (Elshire et al. 2011), multiplexed shotgun genotyping (Andolfatto et al. 2011), double digest RAD-seq (Peterson et al. 2012), and ezRAD (Toonen et al. 2013). This choice determines the number of RAD markers that can be obtained, the amount of sequencing needed for a desired coverage level, the number of samples that can be multiplexed, the monetary cost, and ultimately the success of a project. It has been widely suggested that the number of restriction sites in a genome, for a given enzyme, can be roughly predicted using simple probability, if one has an estimate of the genome size and guanine-cytosine (GC) composition (Baird et al. 2008; Davey et al. 2011). Both of these parameters can be approximated in nonmodel organisms through sequencing-independent techniques such as flow cytometry (Vinogradov 1994, 1998; Šmarda et al. 2011). However, preliminary evidence suggests that there can be significant departures from expectations for particular combinations of taxa and restriction enzymes (Davey \& Blaxter 2011; Davey et al. 2011). 
Type II restriction enzymes, which are endonucleases chiefly produced by prokaryotic microorganisms, cleave double stranded DNA (dsDNA) at specific unmethylated recognition sequences that are 4 to 8 base pairs long and typically palindromic. These enzymes are thought to play an important role as defense systems against foreign phage dsDNA during infection or as selfish parasitic elements, and therefore have been the center of an evolutionary 'arms race' (Rambach \& Tiollais 1974; Karlin et al. 1992; Rocha et al. 2001). Type II restriction enzymes are not known in eukaryotes and are not used as virulence factors by bacteria to infect eukaryotic hosts. Therefore there are no a priori reasons to believe that recognition sites in eukaryotic genomes are subject to selective pressures, but rather they should be evolutionarily neutral. Eukaryotic genomes have heterogeneous compositions with characteristic signatures at the level of diand trinucleotides that are largely independent of coding status or function (Karlin \& Mrázek 1997; Karlin et al. 1998; Gentles \& Karlin 2001). Thus, it is possible that genome composition at these levels has a large influence on the abundance of short sequence patterns such as recognition sequences of restriction enzymes.

Here, we test the hypothesis that genome composition can be used to predict the number of restriction sites for a given combination of restriction enzyme and taxon. For this we: i) performed systematic in silico genome-wide surveys of restriction sites for diverse type II restriction enzymes in 434 eukaryotic whole and draft genomes to determine their frequencies across taxa; ii) examined the composition of genomes at the level of di- and trinucleotides to determine patterns of compositional biases among taxa; iii) developed stochastic models based on GC content, and mono-, di- and trinucleotide compositions to predict the frequencies of restriction sites across taxa and diverse kinds of type II restriction enzymes; iv) evaluated the accuracy of the predictive models by comparing the in silico observed frequencies of restriction sites to the expected frequencies predicted by the models. The number of restriction sites in a genome is not the only factor that determines the number of RAD loci that can be recovered experimentally. The architecture of each genome, and in particular the number of repetitive elements and gene duplications, can significantly decrease the number of unambiguous loci obtained via alignment to a reference genome or de novo assembly. To quantify this contribution we assessed the proportion of restriction-site associated DNA tags that can potentially be recovered unambiguously after empirical sequencing. The software here developed, PredRAD (https://github.com/phrh/PredRAD), and the resulting databases constitute a reference resource that will help guide the choice of restriction enzyme for any study using RAD-related methods. 


\section{RESULTS}

\section{Observed frequencies of restriction sites}

To explore restriction site frequencies across the Eukaryotic tree of life we surveyed recognition sequences for 18 commonly used palindromic type II restriction enzymes in 434 whole and draft genomes. Observed frequencies of restriction sites were highly variable among broad taxonomic groups for the set of restriction enzymes here examined (Table 1) - except for FatI - with clear clustering patterns determined by phylogeny (Fig. 1). For example, with NgoMIV we observed 45.8 restriction sites per megabase $(\mathrm{RS} / \mathrm{Mb}) \pm 24.6($ mean $\pm \mathrm{SD})$ in core eudicot plants, compared to $277.4 \pm 131.3 \mathrm{RS} / \mathrm{Mb}$ in commelinid plants (monocots). Among closely-related species the frequency patterns were similar and variability generally small. Observed frequencies of $\mathrm{RS} / \mathrm{Mb}$ were inversely proportional to the length of the recognition sequence, with orders of magnitude differences among the 4-, 6-, and 8- cutters when compared within the same species. For example, in the starlet anemone Nematostella vectensis there were 3917.6, 167.6, and 6.9 RS/Mb for the 4-cutter FatI, 6-cutter PstI and 8-cutter SbfI, respectively. In contrast, nucleotide composition of the recognition sequence did not show a clear correlation with the observed frequency of restriction sites. For example, 83.6 RS/Mb \pm 25.1 were observed in Neopterigii vertebrates for KpnI (GGTACC) and 622.6 RS/Mb \pm 119.1 were observed for PstI (CTGCAG), both recognition sequences with a GC content of $66.7 \%$.

\section{Dinucleotide compositional biases}

Dinucleotide odds ratios $\left(\bar{\rho}_{X Y}^{*}\right)$ (Burge et al. 1992), a measurement of relative dinucleotide abundances given observed component frequencies used to explore genomic compositional biases, revealed significant compositional biases for all possible dinucleotides (Fig. 2). Both dinucleotides and trinucleotides are considered significantly underrepresented if the odds ratio is $\leq 0.78$, significantly overrepresented if $\geq 1.23$, and equal to expectation if $=1$ (Karlin et al. 1998). The dinucleotide compositional biases were highly variable among broad taxonomic groups (e.g., core eudicot plants) but generally similar within. Two dinucleotide complementary pairs, CG/GC and AT/TA, had highly dissimilar relative frequencies between the members of each pair. The largest biases were for CG, being significantly underrepresented in groups like core eudicot plants $\left(\bar{\rho}_{X Y}^{*}=0.68 \pm 0.11\right)$, gnathostomate vertebrates $\left(\bar{\rho}_{X Y}^{*}=0.32 \pm 0.12\right)$, the Pucciniales rust fungi $\left(\bar{\rho}_{X Y}^{*}=0.66 \pm 0.08\right)$, gastropod mollusks $\left(\bar{\rho}_{X Y}^{*}=0.68, \mathrm{SD}=0.01\right)$, the Trebouxiophyceae green algae $\left(\bar{\rho}_{X Y}^{*}=0.61 \pm 0.19\right)$ and the Saccharomycetales yeast $\left(\bar{\rho}_{X Y}^{*}=0.78 \pm 0.17\right)$. CG was significantly overrepresented in groups like the Apocrita insects 
$\left(\bar{\rho}_{X Y}^{*}=1.59 \pm 0.18\right)$. The complementary dinucleotide GC was not particularly underrepresented in any broad taxonomic group, but tended towards overrepresentation in ecdysozoan invertebrates $\left(\bar{\rho}_{X Y}^{*}=1.24 \pm\right.$ 0.12), being significant in several arthropod and nematode species. Other taxa that showed significant overrepresentation of GC dinucleotides included the Trebouxiophyceae $\left(\bar{\rho}_{X Y}^{*}=1.39 \pm 0.04\right)$ and microsporidia fungi $\left(\bar{\rho}_{X Y}^{*}=1.28 \pm 0.17\right)$. Relative abundances of the dinucleotide AT were within expectations for all eukaryotes, except for the fungus Sporobolomyces roseus $\left(\rho_{X Y}^{*}=0.78\right)$. Contrastingly, the TA dinucleotide tended towards underrepresentation throughout the eukaryotes $\left(\bar{\rho}_{X Y}^{*}=0.8 \pm 0.13\right)$, except in a few hypocreomycetid fungal species, for which it was significantly underrepresented. The TA dinucleotide was significantly underrepresented in trypanosomatids $\left(\bar{\rho}_{X Y}^{*}=0.59 \pm 0.03\right)$, choanoflagellids $\left(\bar{\rho}_{X Y}^{*}=0.43 \pm 0.09\right)$, chlorophytes $\left(\bar{\rho}_{X Y}^{*}=0.62 \pm 0.15\right)$, stramenopiles $\left(\bar{\rho}_{X Y}^{*}=0.70 \pm 0.07\right)$, and marginally underrepresented in most euteleostei fish $\left(\bar{\rho}_{X Y}^{*}=0.77 \pm 0.04\right)$, archosaurs $\left(\bar{\rho}_{X Y}^{*}=0.76 \pm 0.03\right)$ and the Basidiomycota $\left(\bar{\rho}_{X Y}^{*}=0.74 \pm 0.09\right)$, among others.

The remaining dinucleotides had identical relative frequencies between the members of each complementary pair. The dinucleotide pair $\mathrm{GG} / \mathrm{CC}$ was marginally underrepresented in most eukaryotes $\left(\bar{\rho}_{X Y}^{*}=0.88 \pm 0.15\right)$. In the Sarcopterygii vertebrates $\left(\bar{\rho}_{X Y}^{*}=1.02 \pm 0.06\right)$ and embryophyte plants $\left(\bar{\rho}_{X Y}^{*}=1.03\right.$ $\pm 0.07) \mathrm{GG} / \mathrm{CC}$ relative frequencies closely conformed to expectation, whereby $\mathrm{GG} / \mathrm{CC}$ was significantly overrepresented in handful of isolated ecdysozoan, microsporidia and alveolate species, and significantly underrepresented in chlorophytes $\left(\bar{\rho}_{X Y}^{*}=0.72, \mathrm{SD}=0.11\right)$, oomycetes $\left(\bar{\rho}_{X Y}^{*}=0.71 \pm 0.05\right)$, and in several species of the Basidiomycota and the Dothideomycetes. Only the choanoflagellate Salpingoeca and the green alga Asterochloris presented a marginally significant bias for the dinucleotide pair AA/TT ( $\rho_{X Y}^{*}=0.77$ and 0.75 respectively). Similarly, Salpingoeca was the only taxon to show a significant bias for $\mathrm{AC} / \mathrm{GT}\left(\rho_{X Y}^{*}=1.42\right)$. Dinucleotide pair $\mathrm{CA} / \mathrm{TG}$ was among the pairs with largest biases. Significant overrepresentation of $\mathrm{CA} / \mathrm{TG}$ was found in several groups with large CG underrepresentation such as gnathostomates $\left(\bar{\rho}_{X Y}^{*}=1.31 \pm 0.05\right)$, gastropods $\left(\bar{\rho}_{X Y}^{*}=1.29 \pm 0.05\right)$, the Pucciniales $\left(\bar{\rho}_{X Y}^{*}=1.27 \pm 0.02\right)$, the Trebouxiophyceae $\left(\bar{\rho}_{X Y}^{*}=1.62 \pm 0.14\right)$, as well as several species of core eudicots and the Saccharomycetales. Other groups with significant CA/TG overrepresentation include onchocercid nematodes $\left(\bar{\rho}_{X Y}^{*}=1.26 \pm 0.01\right)$, the Ustilaginomycotina fungi $\left(\bar{\rho}_{X Y}^{*}=1.28 \pm 0.05\right)$, trypanosomatids $\left(\bar{\rho}_{X Y}^{*}=1.25 \pm 0.04\right)$, and amoebozoans $\left(\bar{\rho}_{X Y}^{*}=1.33 \pm 0.06\right)$. Overrepresentation biases for the AG/CT 
dinucleotide pair were only present in amniotes $\left(\bar{\rho}_{X Y}^{*}=1.26 \pm 0.02\right)$, the Sporidiobolales fungi $\left(\bar{\rho}_{X Y}^{*}=1.24\right.$ $\pm 0.01)$, and oxytrichid alveolates $\left(\bar{\rho}_{X Y}^{*}=1.24 \pm 0.04\right)$, and other isolated species. Most of these taxa also had large CG underrepresentation. Lastly, most eukaryotes had GA/TC relative frequencies that conformed to expectations, except for few scattered species and small groups such as the Microbotryomycetes fungi $\left(\bar{\rho}_{X Y}^{*}=1.45 \pm 0.13\right)$, the Mamiellales green algae $\left(\bar{\rho}_{X Y}^{*}=1.40 \pm 0.08\right)$, and the Eimeriorina alveolates $\left(\bar{\rho}_{X Y}^{*}=1.26 \pm 0.02\right)$.

\section{Trinucleotide compositional biases}

Trinucleotide odds ratios $\left(\gamma_{X Y Z}^{*}\right)$ (Burge et al. 1992) are another important measurement used to explore genomic compositional biases. Among the examined taxa, these ratios revealed compositional biases for most possible trinucleotides (Fig. 3). However, most of these biases were only significant in scattered individual species (Fig. 4). Among the trinucleotide pairs with significant underrepresentation, CTA/TAG and CGA/TCG showed the most definite broad taxonomic patterns. CTA/TAG was significantly underrepresented in most taxa, except for groups like commelinid plants (monocots) $\left(\gamma_{X Y Z}^{*}=0.87 \pm 0.03\right)$, most core eudicots $\left(\gamma_{X Y Z}^{*}=0.81 \pm 0.02\right)$, eleutherozoans $\left(\gamma_{X Y Z}^{*}=0.82 \pm 0.01\right)$, molluscs $\left(\gamma_{X Y Z}^{*}=0.83 \pm 0.01\right)$, and gnathostomates $\left(\gamma_{X Y Z}^{*}=0.82 \pm 0.02\right)$ - exclusive of the chimaera Callorhinchus milii. Contrastingly, the trinucleotide CGA/TCG was only significantly underrepresented in most tetrapod vertebrates $\left(\gamma_{X Y Z}^{*}=0.82 \pm 0.02\right)$ - exclusive of muroid rodents, bovid ruminants and the Afrotheria - a group containing aarvdvarks, hyraxes, and elephants.

The largest and more widespread overrepresentation biases were for the trinucleotide pair AAA/TTT, being significant in most eukaryotes, except for the majority of the Dikarya fungi $\left(\gamma_{X Y Z}^{*}=1.18 \pm 0.07\right)$. The trinucleotide pairs TAA/TTA and AAT/ATT were significantly overrepresented in many metazoan taxa, particularly in the Neopterygii vertebrates $\left(\gamma_{X Y Z}^{*}=1.3 \pm 0.05\right.$, and $\gamma_{X Y Z}^{*}=1.26 \pm 0.05$ respectively). $\mathrm{AAG} / \mathrm{CTT}$ was significantly overrepresented in the Bacillariophyta diatoms $\left(\gamma_{X Y Z}^{*}=1.24 \pm 0.03\right)$, oomycetes $\left(\gamma_{X Y Z}^{*}=1.28 \pm 0.02\right)$, and the Saccharomycetales $\left(\gamma_{X Y Z}^{*}=1.26 \pm 0.04\right)$. Lastly, CCA/TTG was significantly overrepresented in several tetrapod groups, including the Laurasiatheria - exclusive of the Chiroptera $-\left(\gamma_{X Y Z}^{*}=1.25 \pm 0.02\right)$ and Hominoidea $\left(\gamma_{X Y Z}^{*}=1.23 \pm 0.004\right)$. 
Table 1. Restriction enzymes included in this study.

\begin{tabular}{|c|c|c|c|c|}
\hline $\begin{array}{l}\text { Core } \\
\text { Sequence }\end{array}$ & $\begin{array}{l}\text { Restriction } \\
\text { Enzyme }\end{array}$ & $\begin{array}{l}\text { Recognition } \\
\text { Sequence }\end{array}$ & $\begin{array}{l}\text { Recognition } \\
\text { Sequence } \\
\text { Length }\end{array}$ & $\begin{array}{l}\text { GC Content of } \\
\text { Recongition } \\
\text { Sequence }\end{array}$ \\
\hline \multicolumn{5}{|l|}{ GGCC } \\
\hline & NotI & GCGGCCGC & 8 & 100.0 \\
\hline CCGG & SgrAI & CRCCGGYG & 8 & 87.5 \\
\hline & BsrFI & RCCGGY & 6 & 83.3 \\
\hline & NgoMIV & GCCGGC & 6 & 100.0 \\
\hline & AgeI & ACCGGT & 6 & 66.7 \\
\hline & MspI & CCGG & 4 & 100.0 \\
\hline \multicolumn{5}{|l|}{ TGCA } \\
\hline & SbfI & CCTGCAGG & 8 & 75.0 \\
\hline & PstI & CTGCAG & 6 & 66.7 \\
\hline & NsiI & ATGCAT & 6 & 33.3 \\
\hline \multicolumn{5}{|l|}{ AATT } \\
\hline & ApoI & RAATTY & 6 & 16.7 \\
\hline & EcoRI & GAATTC & 6 & 33.3 \\
\hline & MluCI & AATT & 4 & 0.0 \\
\hline \multicolumn{5}{|l|}{ TTAA } \\
\hline & MseI & TTAA & 4 & 0.0 \\
\hline \multicolumn{5}{|l|}{ CATG } \\
\hline & $\begin{array}{l}\text { Nspl } \\
\text { NcoI }\end{array}$ & $\begin{array}{l}\text { RCATGY } \\
\text { CCATGG }\end{array}$ & $\begin{array}{l}6 \\
6\end{array}$ & $\begin{array}{l}50.0 \\
66.7\end{array}$ \\
\hline & PciI & ACATGT & 6 & 33.3 \\
\hline & FatI & CATG & 4 & 50.0 \\
\hline \multicolumn{5}{|l|}{ GTAC } \\
\hline & KpnI & GGTACC & 6 & 66.7 \\
\hline
\end{tabular}




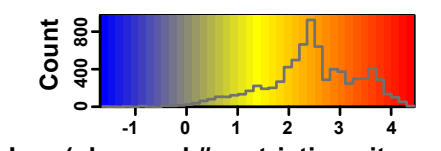

Log (observed \# restriction sites per Mb)

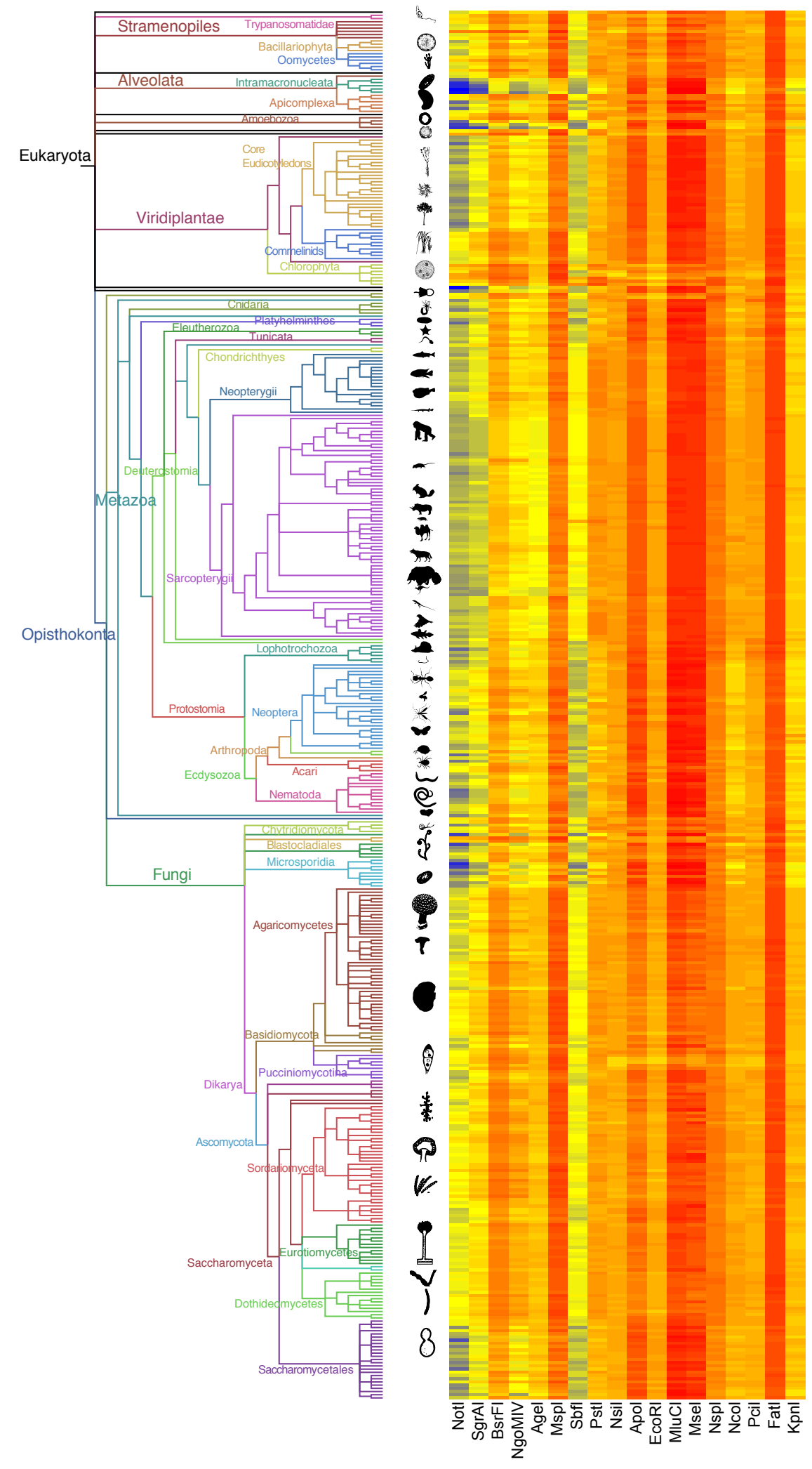


Figure 1. Observed restriction site frequencies. Left: phylogenetic tree of all eukaryotic taxa analyzed in this study. The tree is based on the NCBI taxonomy tree retrieved on May 16, 2013 using the iTOL tool http://itol.embl.de (Letunic \& Bork 2011). Branch colors and labels indicate broad taxonomic groups. Organism silhouettes and cartoons were created by the authors or obtained from http://phylopic.org/. Right: heatmap of the observed frequency of restriction sites. Each row corresponds to a species from the tree on the left, and each column corresponds to a different restriction enzyme. Gray line in the color-scale box shows the distribution histogram of all values. 

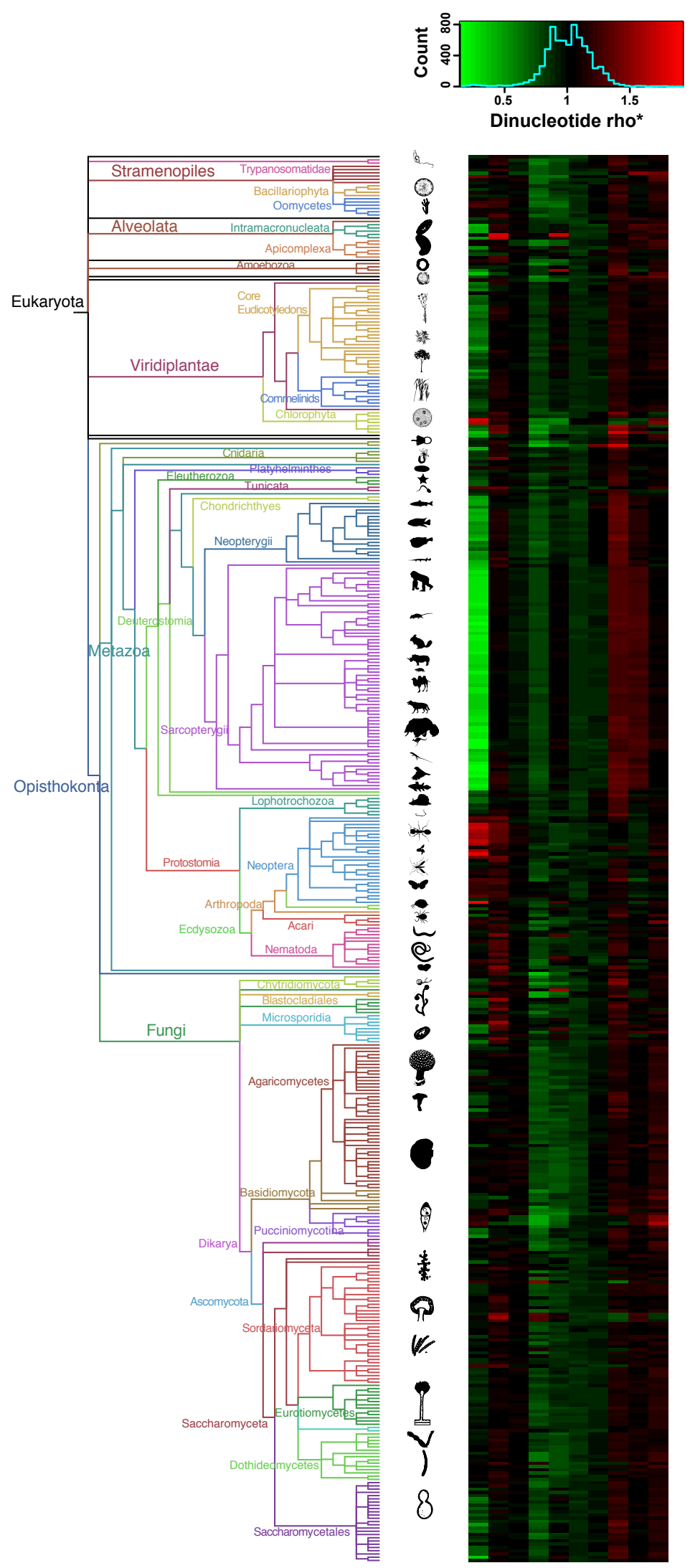

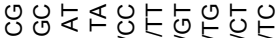

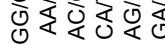
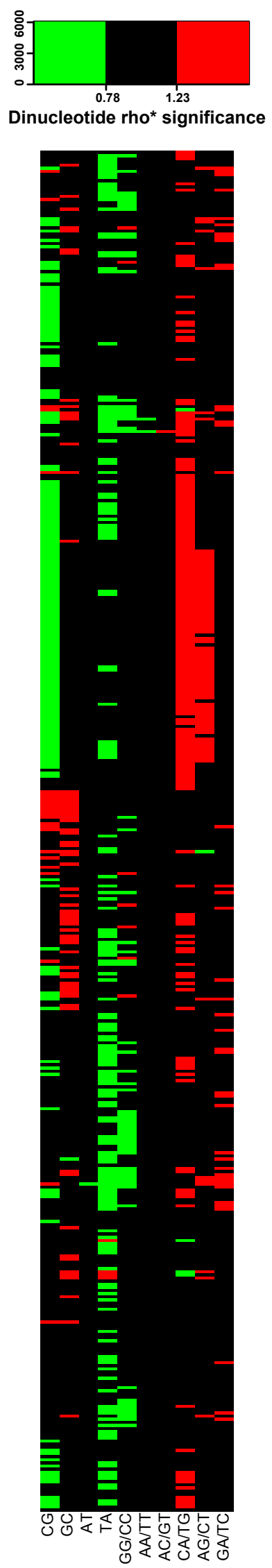
Figure 2. Dinucleotide compositional biases and significances. Left: phylogenetic tree as in Fig. 1. Center: heatmap of the $\rho_{X Y}^{*}$ odds ratio values. Right: heatmap of the $\rho_{X Y}^{*}$ odds ratio significant values $\rho_{X Y}^{*}<0.78$ and $\rho_{X Y}^{*}>1.23$. Each row corresponds to a species from the tree on the left, and each column corresponds to a different dinucleotide. Green indicates underrepresentation and red indicates overrepresentation. Cyan line in the color-scale box shows the distribution histogram of all values. 

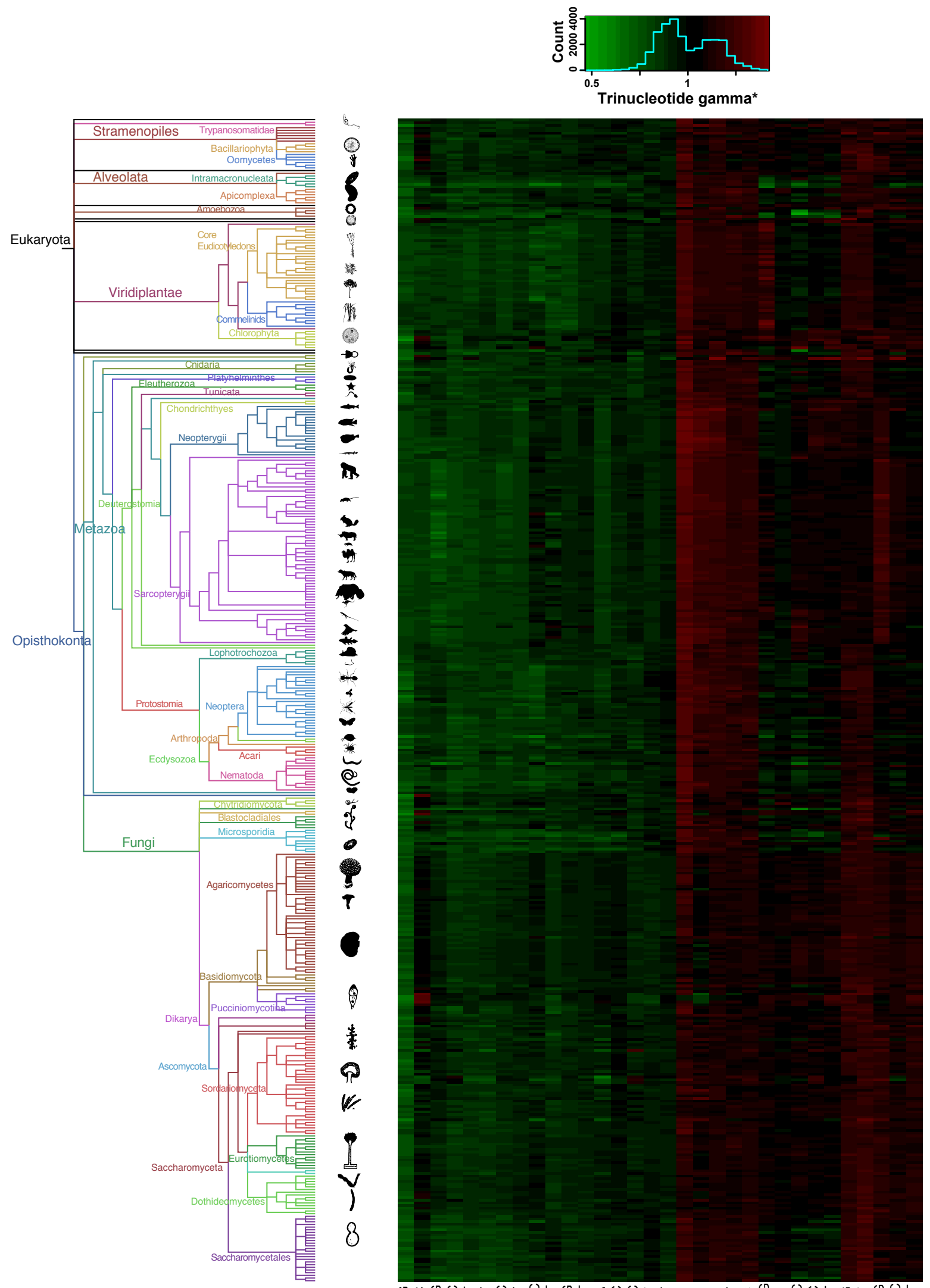

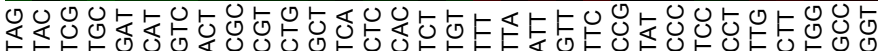

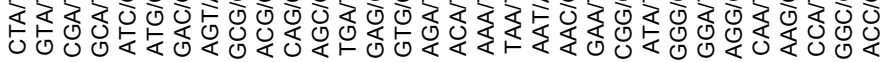


Figure 3. Trinucleotide compositional biases. Left: phylogenetic tree as in Fig. 1. Right: heatmap of the $\gamma_{X Y Z}^{*}$ odds ratio values. Each row corresponds to a species from the tree on the left, and each column corresponds to a different trinucleotide. Green indicates underrepresentation and red indicates overrepresentation. Cyan line in the color-scale box shows the distribution histogram of all values. 

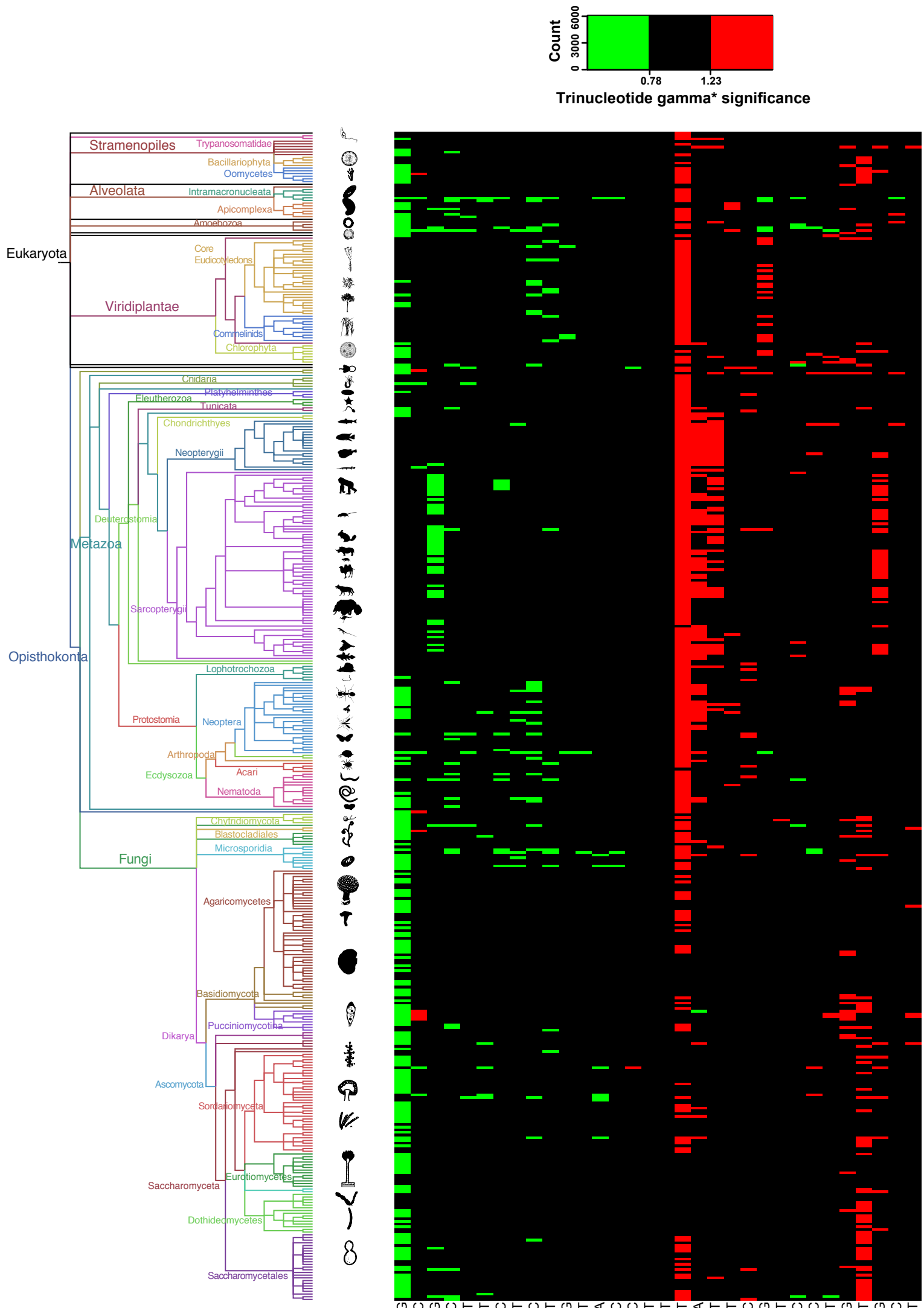

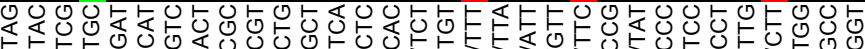

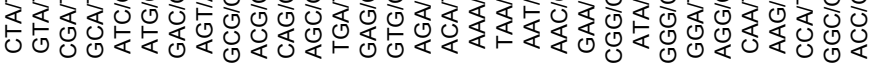


Figure 4. Trinucleotide compositional biases significances. Left: phylogenetic tree as in Fig. 1. Right: heatmap of the $\gamma_{X Y Z}^{*}$ odds ratio significant values $\rho_{X Y}^{*}<0.78$ and $\rho_{X Y}^{*}>1.23$. Each row corresponds to a species from the tree on the left, and each column corresponds to a different trinucleotide. Green indicates underrepresentation and red indicates overrepresentation. Cyan line in the color-scale box shows the distribution histogram of all values.

\section{Expected frequencies of restriction sites}

To test the hypothesis that compositional heterogeneity in eukaryotic genomes can determine the frequency of restriction sites of each genome we developed probability models based on the GC content of each genome, as well as the mononucleotide, dinucleotide and trinucleotide compositions to predict the expected frequency of recognition sequences for each restriction enzyme. We evaluated the fit of each model using a similarity index (SI), defined as the quotient of the number of observed and expected restriction sites, minus one. A positive $S I$ indicates that the number of observed restriction sites is greater than the expected, whereas a negative $S I$ indicates a smaller number of observed sites than expected. If $S I$ is equal to 0 , then the number of observed sites is equal to the expectation. For example, a $S I=1$ indicates that the number of observed restriction sites for a particular enzyme in a given genome is twice the number of expected sites predicted by a particular model. Trinucleotide composition models were in general a better predictor, in terms of their accuracy and precision, of the expected number of restriction sites than any of the other models (Fig. 5, Fig. 6). The mononucleotide and GC content models produced relatively poor predictions that were indistinguishable from one another (Fig. 5, Fig. 6). In a few cases the other models outperformed the trinucleotide model, e.g., EcoRI (Fig. 5, Fig. 6, Fig. 7). The fit of the predictions was highly variable among broad taxonomic groups but generally similar within, e.g., in Neopterigii vertebrates an average SI of $0.14 \pm 0.19$ for AgeI with the dinucleotide model, compared to $0.31 \pm 0.19$ in Sarcopterigii.

\section{Recovery of RAD-tags after in silico sequencing}

In most cases, the recovery of RAD-tags after in silico sequencing was notably high, with a median percentage of suppressed alignments to the reference genome assembly of only $3 \%$ (Fig. 8). There was no evident recovery bias by restriction enzyme, but rather bias was pronounced in a few individual species, likely indicating an enrichment of repetitive regions or duplications. 

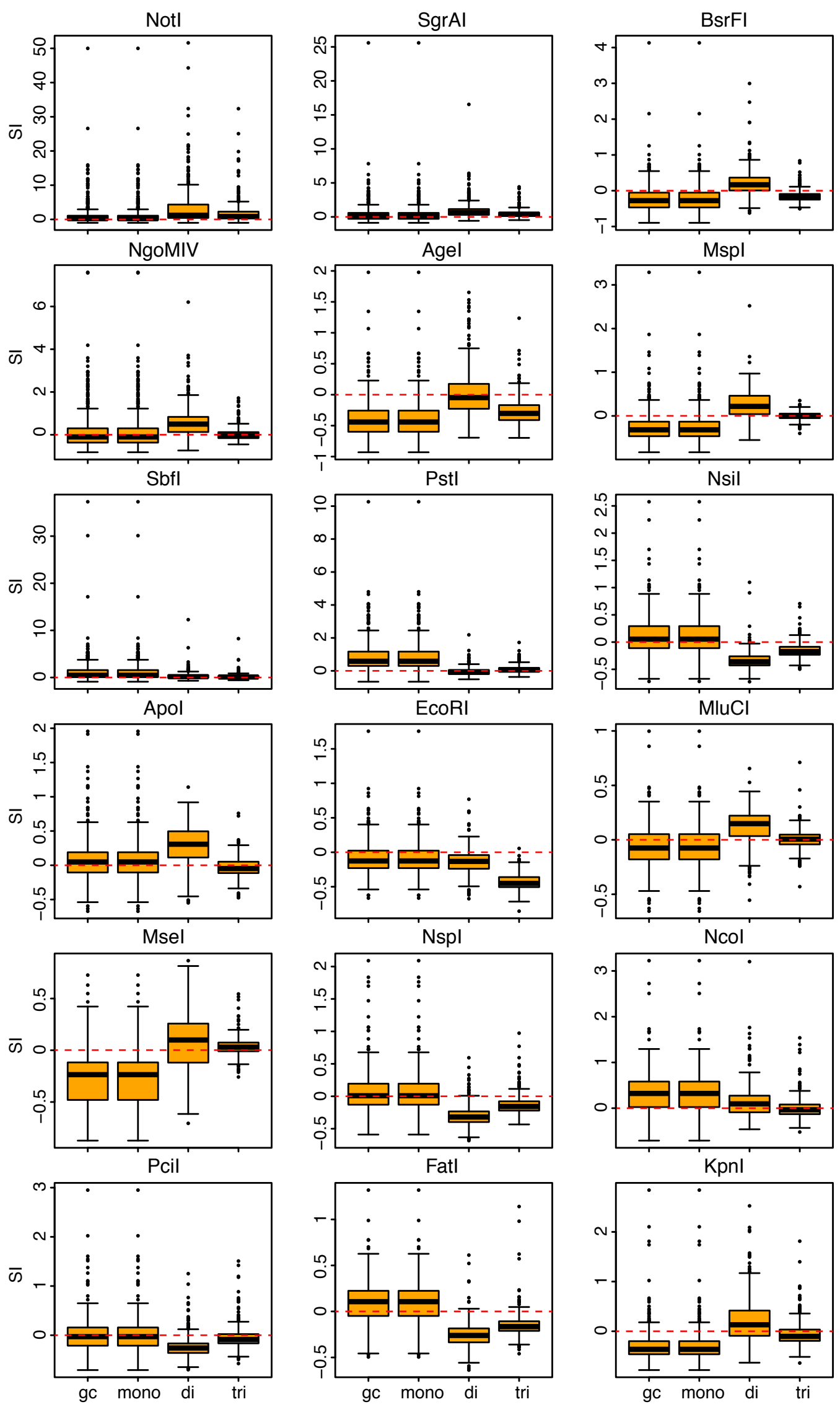
Figure 5. Overall fit of genome composition models per restriction enzyme. Vertical axes in the box and whisker plots indicate the values of the similarity index $(S I)$ for each species per enzyme (see Methods section). Horizontal axes in the box and whisker plots indicate the genome composition model: GC content (gc), mononucleotide (mono), dinucleotide (di), and trinucleotide (tri). Horizontal edges of range boxes indicate the first and third quartiles of the $S I$ values under each composition model. The thick horizontal black line represents the median. Whiskers indicate the value of 1.5 times the inter-quartile range from the first and third quartiles. Outliers are defined as SI values outside the whiskers range and are represented by dots. Outlier value of Entamoeba histoyitica for NotI was excluded. Red dotted lines indicate $S I=0$. 


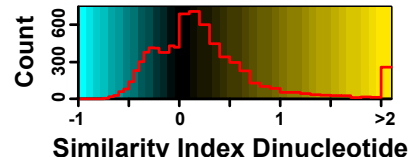

Similarity Index Dinucleotide

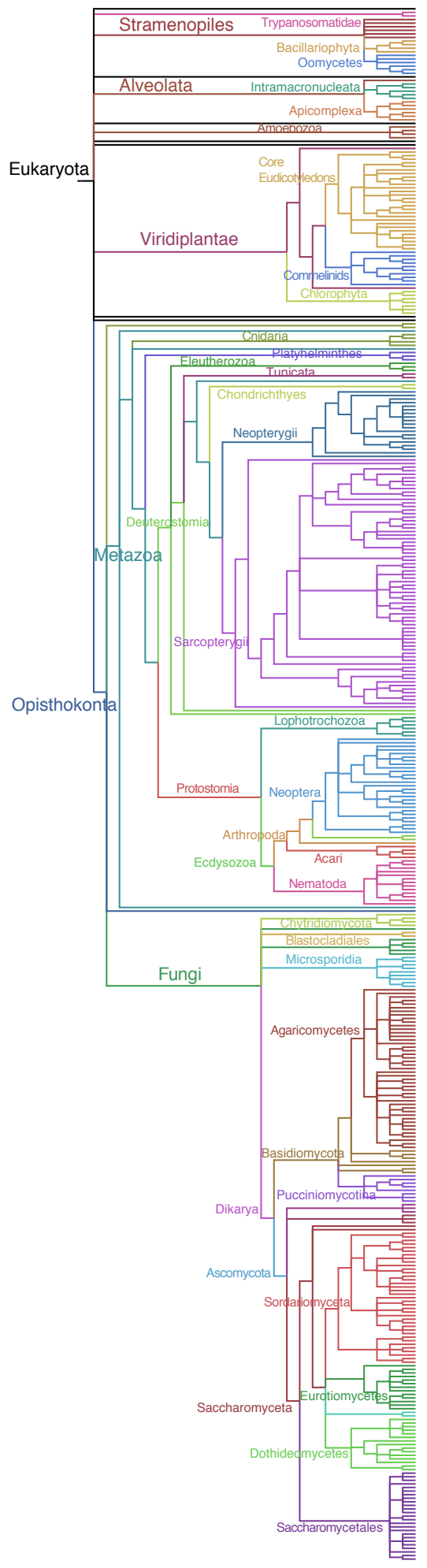

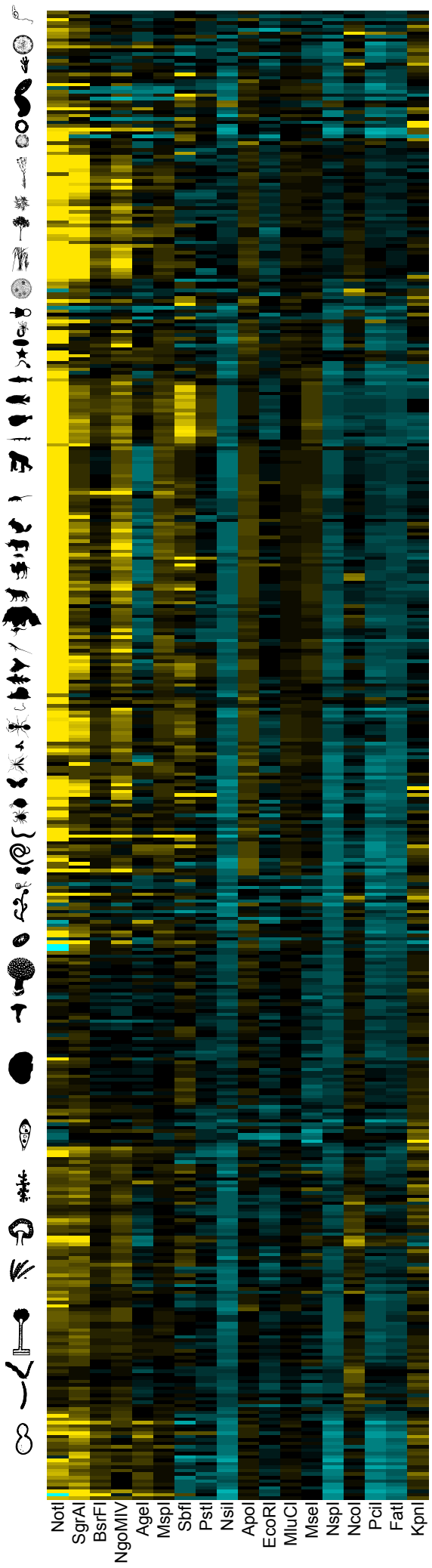
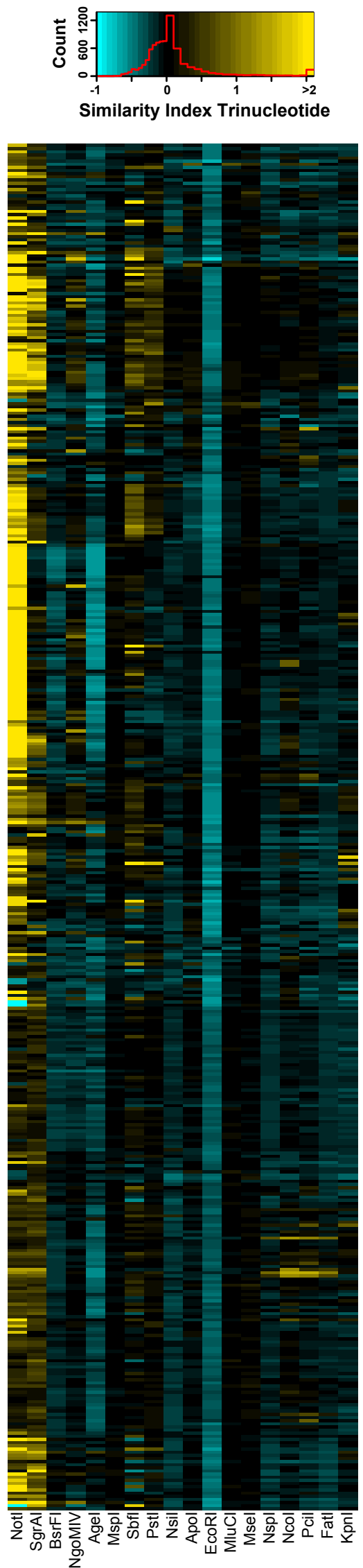
Figure 6. Similarity indexes for dinucleotide and trinucleotide genome composition models. Left: phylogenetic tree as in Fig. 1. Center: heatmap of the similarity indexes for the dinucleotide model Right: heatmap of the similarity indexes for the trinucleotide model. Each row corresponds to a species from the tree on the left, and each column corresponds to a different restriction enzyme. Cyan indicates $S I<0$ and yellow indicates $S I>0$. Red line in the color-scale box shows the distribution histogram of all values. 


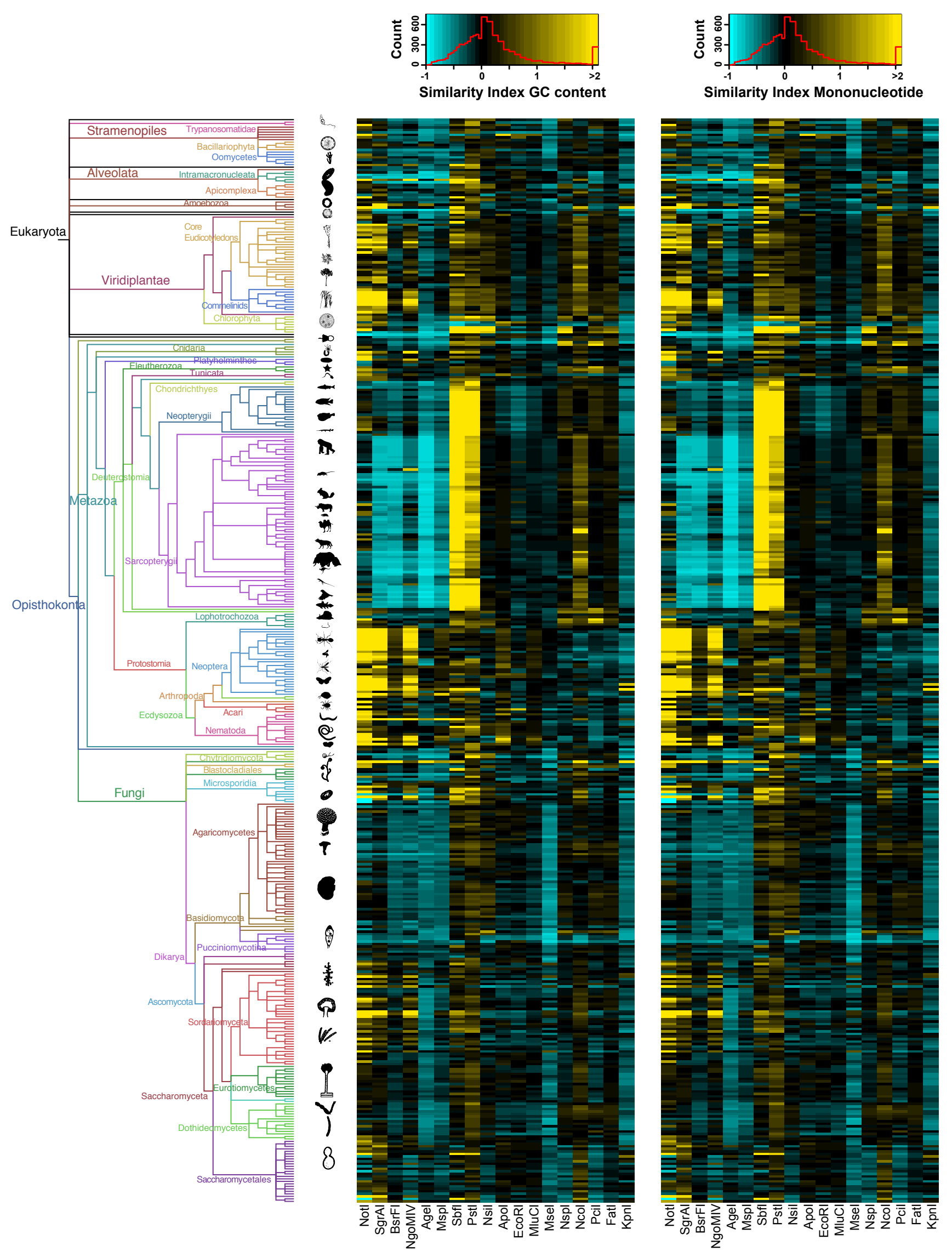


Figure 7. Similarity indexes for GC content and mononucleotide genome composition models. Left: phylogenetic tree as in Fig. 1. Center: heatmap of the similarity indexes for the GC content model Right: heatmap of the similarity indexes for the mononucleotide model. Each row corresponds to a species from the tree on the left, and each column corresponds to a different restriction enzyme. Cyan indicates $S I<0$ and yellow indicates $S I>0$. Red line in the color-scale box shows the distribution histogram of all values. 


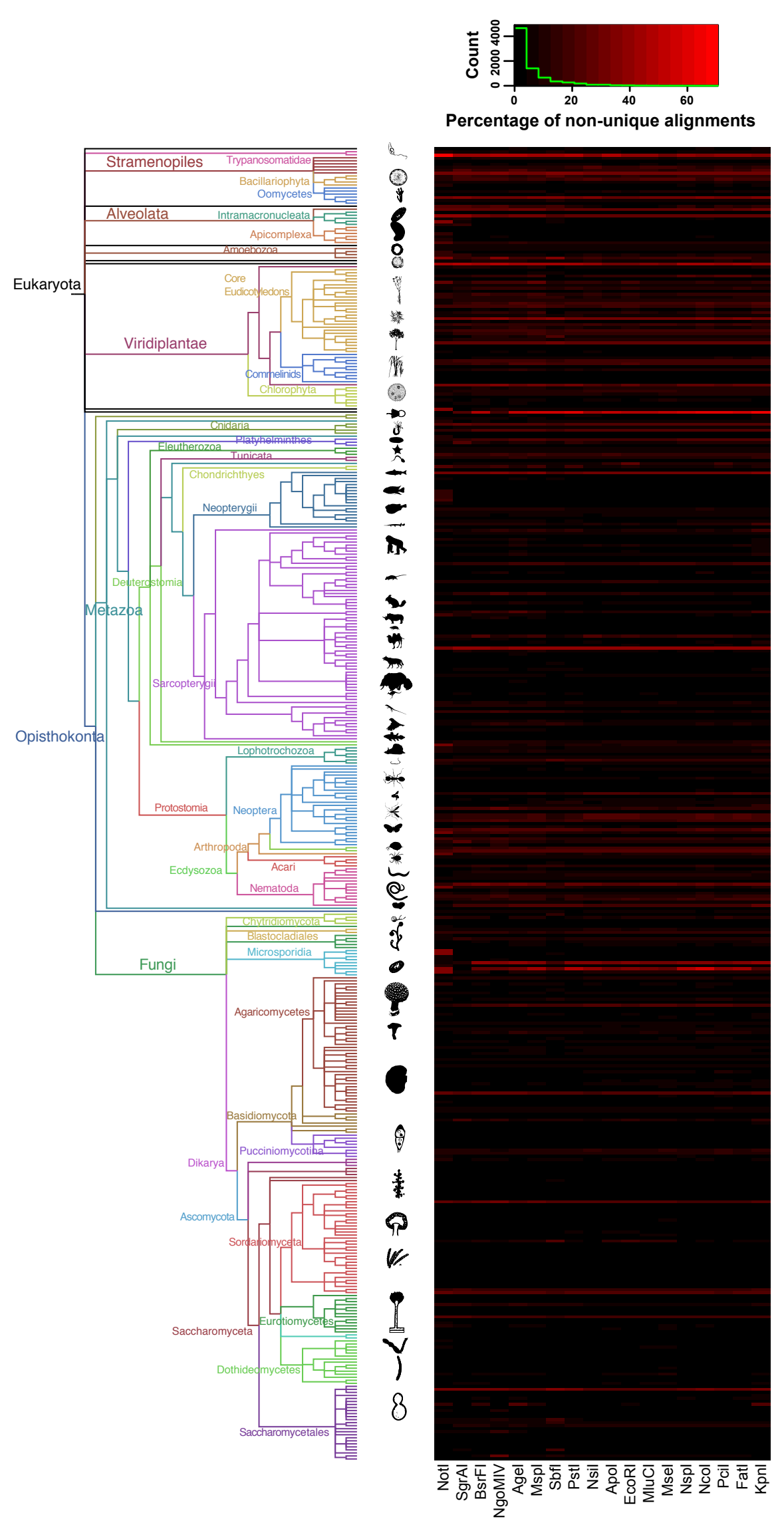


Figure 8. Recovery of RAD-tags after in silico genome digestion and sequencing. Left: phylogenetic tree as in Fig. 1. Right: heatmap of the percentage of RAD-tags that produced more than one unique alignment to their reference genome. Each row corresponds to a species from the tree on the left, and each column corresponds to a different restriction enzyme. Green line in the color-scale box shows the distribution histogram of all values.

\section{DISCUSSION}

\section{Genome-wide surveys of restriction sites}

Observed cut frequencies for a given restriction enzyme are strikingly variable among broad eukaryotic taxonomic groups, but similar among closely-related species. This is consistent with the hypothesis that the abundance of restriction sites is largely determined by phylogenetic relatedness. This pattern is most evident in groups that have a larger taxonomic representation, such as mammals. As more genome assemblies become available the pattern resolution will become clearer in many other underrepresented taxonomic groups, and through the use of comparative methods in a robust phylogenetic framework, it will be possible to establish taxon-specific divergence thresholds diagnostic of significant evolutionary changes in genome architecture.

As expected, observed frequencies of restriction sites with shorter recognition sequences are generally higher than the observed frequencies with longer recognition sequences. However this pattern in not universal. There are several instances in which the frequency of restriction sites for a high-denomination cutter is higher than for a low-denomination cutter. For example, in primates the frequency of the 8-cutter SbfI $(24.6 \pm 1.7 \mathrm{RS} / \mathrm{Mb})$ is significantly higher than the frequency of the 6-cutter AgeI $(18.4 \pm 1.4$ $\mathrm{RS} / \mathrm{Mb}$ ). These deviations from expectation are indicative of enzyme-specific frequency biases for particular taxa, and, as illustrated in the results section, are not correlated with the base composition of recognition sequences.

\section{Genomic compositional biases}

Our analyses indicate that there are significant compositional biases for most dinucleotides and trinucleotides across the eukaryotes. Many of these biases are significant only within individual species scattered throughout the eukaryotic tree of life. However, there are several particular dinuclotides and trinucleotides that show significant biases across the eukaryotic tree of life. Our observation that these biases are highly variable among broad taxonomic groups but generally similar within is congruent with findings from previous studies (e.g., Gentles \& Karlin 2001). The most obvious biases across taxa are observed in the gnatostomate vertebrates; however, this is most likely due to rampant undersampling in 
most other groups of eukaryotes (vertebrate genome assemblies represent $21 \%$ of all the taxa in this study).

The dinucleotides $\mathrm{CG}, \mathrm{GC}$, TA, and $\mathrm{CA} / \mathrm{TG}$ show the most conspicuous bias patterns across the eukaryotic tree of life. Biases in most of these dinucleotides are likely linked to important biological processes. Notably the underrepresented dinucleotide CG is a widely known target for methylation related to transcriptional regulation (Bird 1980) and retrotransposon inactivation (Yoder et al. 1997) in vertebrates and eudicots. The corresponding overrepresentation of AG/CT fits the classic model of "methylation-deamination-mutation" by which a methylated cytosine in the CG pair tends to deaminate when unpaired and mutate into a thymidine with a corresponding CA complement. Interestingly CG and GC dinucleotides are significantly overrepresented in several groups of apocritic insects, as well as in some fungi and single-cell eukaryotes. CG is not a primary target for methylation in Drosophila (Lyko et al. 2000), instead CT, and in lesser degree CA and CC, are methylated in higher proportion. None of these dinucleotide pairs is significantly underrepresented in apocritic insects. The widespread TA underrepresentation has been traditionally attributed to stop codon biases, thermodynamic instability and susceptibility of UA to cleavage by RNAses in RNA transcripts (Beutler et al. 1989).

The trinucleotides CTA/TAG, AAA/TTT, TAA/TTA, CCA/TGG show the most conspicuous bias patterns across the eukaryotic tree of life. The biases in CTA/TAG have been widely attributed to the stop codon nature of UAG. However, the trinucleotides corresponding to the other stop codons (Burge et al. 1992), UAA and UGA, are overrepresented or not biased across eukaryotes. The reasons behind other cases of trinucleotide biases are less understood.

\section{Predictability of restriction site frequencies}

Our analyses indicate that in most cases the trinucleotide genome composition model is the best predictor, and the GC content and mononucleotide models are the worst predictors of the expected number of restriction sites in a eukaryotic genome. It is possible that the greater number of parameters in the trinucleotide model (64, compared to 16, 4 and 2 of the dinucleotide, mononucleotide and GC content model, respectively) is the cause of the better fit. However, this trend is not universal. As illustrated in the results section, the other models in a few cases outperformed the trinucleotide composition model. Neither the GC content nor length of the recognition sequence can confidently explain the observed discrepancies. It is not surprising that fit of the predictions made by the models is highly variable across taxonomic groups, given the high variability observed in the frequencies of restriction sites and genetic compositions across the eukaryotic tree of life. We conclude that the predictability of restriction site 
frequencies in eukaryotic genomes needs to be treated on a case-specific basis, whereby the phylogenetic position of the taxon of interest and the specific recognition sequence of the selected restriction enzyme are the chief foci among the most determinant factors.

\section{Implications for RAD-seq and related methodologies}

For the design of a study using RAD-seq, or a related methodology, there are two fundamental questions that researchers face: i) what is the best restriction enzyme to use to obtain a desired number of RAD tags in the organism of interest? and ii) how many markers can be obtained with a particular enzyme in the organism of interest? The results from this study coupled with the developed software pipeline PredRAD, will allow any researcher to obtain an approximate answer to these questions.

In a best-case scenario for the practical design of a study using RAD-seq, or a related methodology, the species of interest is already included in the database presented here. In this case, the best proxy for the estimated number of RAD tags that could be obtained empirically would be twice the number of in silico observed restriction sites for each restriction enzyme (each restriction site is expected to produce two RAD tags, one in each direction from the restriction site) minus the number of suppressed read alignments to the reference genome assembly. For example, the predicted number of RAD tags for SbfI in starlet anemone Nematostella vectensis is 3,370 , being highly similar to the range of RAD tags $(2,300$ 2,800) obtained empirically by Reitzel et al. (2013). For library preparation protocols in which a fragment size selection step is done without a prior shearing step, e.g., ddRAD (Peterson et al. 2012) and ezRAD (Toonen et al. 2013), the size.select function of the software package SimRAD (Lepais \& Weir 2014) constitutes a valuable complementary study-design tool. If a new genome assembly becomes available for the target species and/or the researcher wishes to evaluate an additional restriction enzyme, PredRAD can be re-executed with these data to quantify the number of restriction sites and the recovery potential, as well as to estimate the probability of the new recognition sequence based on genome composition models.

In the scenario that the genome sequence of the species of interest is not available, the best alternative is to look at the closest relative with a genome assembly. A range of approximate values for the number of RAD tags can be obtained from i) the number of in silico observed restriction sites in the closely related species; ii) the frequency of restriction sites in the closely related species, and the genome size of the species of interest; and iii) the probability of the recognition sequence for the enzyme(s) based on the best-fit genome composition model (SI closest to 0 ) from the closely related species and the genome size of the target species. The genome size of the species of interest can be estimated through sequencingindependent techniques such as flow cytometry (Vinogradov 1994, 1998; Šmarda et al. 2011). For 
example, the predicted range in the number of RAD tags that could be obtained using SbfI in thoracican barnacles, a group for which no genome sequence assemblies are available, is 10,000-30,000, based on the observed frequency of the SbfI recognition sequence and its probability using a trinucleotide composition model in the genome of the crustacean Daphnia pulex (ranges of genome size for barnacles were obtained from the Animal Genome Size Database, http://ww.genomesize.com). Herrera et al. (Chapter 3) empirically obtained ca. 18,000 RAD tags for several species in this group. The possibility that the frequency of restriction sites and genome composition can be accurately estimated from alternative datasets such as a transcriptome is worth evaluating during restriction-enzyme selection for taxa without sequenced genomes.

Additional factors that can influence the number of RAD tag markers that can be obtained experimentally, and need to be considered during study design and data analysis steps, include: genome differences among individuals, level of heterozygosity, the amount of methylation in the genome, the number of repetitive regions and gene duplicates present in the target genome, the sensitivity of a particular restriction enzyme to methylation, the efficiency of the enzymatic digestion, the quality of library preparation and sequencing, the amount of sequencing, sequencing and library preparation biases, and the parameters used to clean, cluster and analyze the data, among others (see Davey et al. (2013), (Catchen et al. 2013), DaCosta and Sorenson (2014), and Mastretta-Yanes et al. (2014) for further discussion).

\section{CONCLUSIONS}

In this study we tested the hypothesis that genome composition can be used to predict the number of restriction sites for a given combination of restriction enzyme and genome. Our analyses reveal that in most cases the trinucleotide genome composition model is the best predictor, and the GC content and mononucleotide models are the worst predictors of the expected number of restriction sites in a eukaryotic genome. We conclude that the predictability of restriction site frequencies in eukaryotic genomes needs to be treated on a case-specific basis, whereby the phylogenetic position of the taxon of interest and the specific recognition sequence of the selected restriction enzyme are the chief foci among the most determinant factors. The results from this study and the software developed from it will help guide the design of any study using RAD sequencing and related methods. As more genome assemblies become available in underrepresented taxonomic groups the patterns of compositional biases and restriction site frequencies across the eukaryotic tree of life will become clearer and will improve our understanding of genome evolution. 


\section{METHODS}

\section{Observed frequencies of restriction sites}

Assemblies from eukaryotic whole genome shotgun (WGS) sequencing projects available as of December 2012 were retrieved primarily from the U.S. National Center for Biotechnology Information (NCBI) WGS database (Additional file 1). Only one species per genus was included. Of the 434 genome assemblies included in this study, $42 \%$ corresponded to fungi, $21 \%$ to vertebrates, $16 \%$ invertebrates, and 9\% plants. Only unambiguous nucleotide calls were taken into account. Genome sequence sizes were measured as the number of unambiguous nucleotides in the assembly. A set of 18 commonly used palindromic type II restriction enzymes with variable nucleotide compositions was screened in each of the genome assemblies (Table 1). The number of restriction sites present in each genome was obtained by counting the number of unambiguous matches for each recognition sequence pattern. Under optimal experimental conditions each restriction site should produce two RAD tags, one in each direction from the restriction site. Therefore, we define the number of observed RAD tags in each genome assembly as twice the number of recognition sequence pattern matches.

\section{Expected frequencies of restriction sites}

To test the hypothesis that compositional heterogeneity in eukaryotic genomes can determine the frequency of restriction sites of each genome, we characterized the GC content, as well as the mononucleotide, dinucleotide and trinucleotide compositions of each genome and developed probability models to predict the expected frequency of recognition sequences for each restriction enzyme. GC content was calculated as the proportion of unambiguous nucleotides in the assembly that are either guanine or cytosine, assuming that the frequency of guanine is equal to the frequency of cytosine. Mononucleotide composition was determined as the frequency of each one of the four nucleotides. Dinucleotide and trinucleotide compositions were determined as the frequency of each one of the 16 or 64 possible nucleotide combinations, respectively. The odds ratios proposed by Burge et al. (1992) were used to estimate compositional biases of dinucleotides (1) and trinucleotides (2) across genomes.

$$
\rho_{X Y}^{*}=\frac{f_{X Y}^{*}}{f_{X}^{*} f_{Y}^{*}}
$$

$$
\gamma_{X Y Z}^{*}=\frac{f_{X Y Z}^{*} f_{X}^{*} f_{Y}^{*} f_{Z}^{*}}{f_{X Y}^{*} f_{Y Z}^{*} f_{X N Z}^{*}}
$$


Where $f_{X}^{*}$ is the relative frequency of the mononucleotide $X, f_{X Y}^{*}$ is the relative frequency of the dinucleotide $X Y$, and $f_{X Y Z}^{*}$ is the relative frequency of the trinucleotide $X Y Z$. All frequencies take into account the antiparallel structure of double stranded DNA. $N$ represents any mononucleotide.

Mononucleotide and GC content sequence models were used to estimate the probability of a particular recognition sequence (3) assuming that each nucleotide is independent of the others and of its position on the recognition sequence. The GC content model assumes that the relative frequencies of guanine and cytosine in the genome sequence are equal. This model has only two parameters, the GC and AT frequencies. In the mononucleotide model there are four parameters, one for each of the four possible nucleotides.

$$
p(s)=\prod_{i=1, \ldots, n(s)} p\left(s_{i}\right)
$$

Here, $p\left(s_{i}\right)$ is the probability of nucleotide $s_{i}$ at the position $i$ of the recognition sequence. In the GC content model $p\left(s_{i}\right)$ can take the values of $f_{G, C}$ or $f_{A, T}$. In the mononucleotide model $p\left(s_{i}\right)$ can take the values of $f_{A}, f_{G}, f_{C}$, or $f_{T}$. Where $f_{X}$ is the frequency of a given mononucleotide.

Dinucleotide and trinucleotide sequence models were defined as first and second degree Markov chain transition probability models with 16 or 64 parameters, respectively (Karlin et al. 1992; Singh 2009). These models take into account the position of each nucleotide in the recognition sequence. Nucleotides along the recognition sequence are not independent from nucleotides in neighboring positions. The probability of a particular recognition sequence for these Markov chain models was calculated as:

$$
p(s)=p\left(s_{1}\right) \prod_{i=2, \ldots, n(s)} p_{c}\left(s_{i} \mid s_{i-1}, \ldots, s_{i-n}\right)
$$

Where $p\left(s_{1}\right)$ is the probability at the first position on the recognition sequence and $p_{c}$ is the conditional probability of a subsequent nucleotide on the recognition sequence depending on the previous $n$ nucleotides. In the dinucleotide sequence model $n=1$ and in the trinucleotide sequence models $n=2$. 


\section{Expectations versus observations}

To assess the effectiveness of the predictive recognition sequence models, we compared the number of observed restriction sites in the genome assemblies with the expected number. The expected number of restriction sites in a given genome was calculated as the product of the probability of a recognition sequence multiplied by the genome sequence size. To quantify the departures from expectation, we define a similarity index $(S I)$ as $S I=(O-E) / E$, where $O$ and $E$ are the observed and expected number of restriction sites, respectively. If $S I=0$, then $E=O$. If $S I<0$, then $E>O$, and vice versa.

\section{Recovery of restriction-site associated DNA tags}

To assess the proportion of restriction-site associated DNA tags that can potentially be recovered unambiguously after empirical sequencing, we performed in silico sequencing experiments for all genome assembly-restriction enzyme combinations. For each restriction site located in the genome assemblies, 100 base pairs up- and down-stream of the restriction site were extracted. This sequence read length is typical of sequencing experiments performed with current Hi-Seq platforms (Illumina Inc.). The resulting RAD tags were aligned back to their original genome assemblies using BOWTIE v0.12.7 (Langmead et al. 2009). Only reads that produced a unique best alignment were retained.

The analytical software pipeline here described and output database files are publicly available at https://github.com/phrh/PredRAD.

\section{AUTHOR'S CONTRIBUTIONS}

SH conceived and designed research. SH and PHR developed the software. SH analyzed the data. SH, TMS and HW contributed computing equipment. SH wrote the paper with comments from PHR and TMS.

\section{ACKNOWLEDGEMENTS}

This research was supported by the Office of Ocean Exploration and Research of the National Oceanic and Atmospheric Administration (NA09OAR4320129 to TS); the Division of Ocean Sciences of the National Science Foundation (OCE-1131620 to TS); the Astrobiology Science and Technology for Exploring Planets program of the National Aeronautics and Space Administration (NNX09AB76G to TS); and the Academic Programs Office (Ocean Ventures Fund to SH), the Ocean Exploration Institute 
(Fellowship support to TMS) and the Ocean Life Institute of the Woods Hole Oceanographic Institution (internal grant to TMS and SH). We thank A. Reitzel, A. Tarrant, and C. Dunn for providing helpful discussions. A. Tarrant, A. Reitzel, E. Bors, A. Govindarajan, provided constructive comments on this manuscript.

\section{REFERENCES}

Andersen EC, Gerke JP, Shapiro JA, et al. (2012) Chromosome-scale selective sweeps shape Caenorhabditis elegans genomic diversity. Nature Genetics 44, 285-290.

Andolfatto P, Davison D, Erezyilmaz D, et al. (2011) Multiplexed shotgun genotyping for rapid and efficient genetic mapping. Genome Research 21, 610-617.

Baird N, Etter P, Atwood T, et al. (2008) Rapid SNP discovery and genetic mapping using sequenced RAD markers. PLoS One 3, e3376.

Beutler E, Gelbart T, Han J, Koziol J, Beutler B (1989) Evolution of the genome and the genetic code: Selection at the dinucleo- tide level by methylation and polyribonucleotide cleavage. Proceedings of the National Academy of Sciences of the United States of America 86, 192-196.

Bird AP (1980) DNA methylation and the frequency of Cpg in animal DNA. Nucleic Acids Research 8 , 1499-1504.

Burge C, Campbell AM, Karlin S (1992) Over- and under-representation of short oligonucleotides in DNA sequences. Proceedings of the National Academy of Sciences of the United States of America 89, 1358-1362.

Catchen J, Hohenlohe PA, Bassham S, Amores A, Cresko WA (2013) Stacks: an analysis tool set for population genomics. Molecular Ecology 22, 3124-3140.

DaCosta JM, Sorenson MD (2014) Amplification biases and consistent recovery of loci in a double-digest RAD-seq protocol. PLoS One 9, e106713.

Davey JW, Blaxter ML (2011) RADSeq: Next-generation population genetics. Briefings in Functional Genomics and Proteomics 9, 416-423.

Davey JW, Cezard T, Fuentes-Utrilla P, et al. (2013) Special features of RAD sequencing data: Implications for genotyping. Molecular Ecology 22, 3151-3164.

Davey JW, Hohenlohe PA, Etter PD, et al. (2011) Genome-wide genetic marker discovery and genotyping using next-generation sequencing. Nature Reviews Genetics 12, 499-510.

Eaton DAR, Ree RH (2013) Inferring phylogeny and introgression using RADseq data: an example from flowering plants (Pedicularis: Orobanchaceae). Systematic Biology 62, 689-706.

Elshire RJ, Glaubitz JC, Sun Q, et al. (2011) A robust, simple genotyping-by-sequencing (GBS) spproach for high diversity species. PLoS One 6, e19379.

Emerson KJ, Merz CR, Catchen JM, et al. (2010) Resolving postglacial phylogeography using highthroughput sequencing. Proceedings of the National Academy of Sciences of the United States of America 107, 16196-16200.

Gentles AJ, Karlin S (2001) Genome-scale compositional comparisons in eukaryotes. Genome Research 11, 540-546.

Herrera S, Watanabe H, Shank TM (Chapter 3) Evolutionary and biogeographical patterns of barnacles from deep-sea hydrothermal vents.

Hohenlohe P, Bassham S, Etter P, et al. (2010) Population genomics of parallel adaptation in threespine stickleback using sequenced RAD tags. PLoS Genetics 6, e1000862.

Karlin S, Burge C, Campbell AM (1992) Statistical analyses of counts and distributions of restriction sites in DNA sequences. Nucleic Acids Research 20, 1363-1370. 
Karlin S, Campbell AM, Mrázek J (1998) Comparative DNA analysis across diverse genomes. Annual Review of Genetics 32, 185-225.

Karlin S, Mrázek J (1997) Compositional differences within and between eukaryotic genomes. Proceedings of the National Academy of Sciences of the United States of America 94, 1022710232.

Langmead B, Trapnell C, Pop M, Salzberg SL (2009) Ultrafast and memory-efficient alignment of short DNA sequences to the human genome. Genome Biology 10, R25.

Lepais O, Weir JT (2014) SimRAD: an R package for simulation-based prediction of the number of loci expected in RADseq and similar genotyping by sequencing approaches. Molecular Ecology Resources 14, 1314-1321.

Letunic I, Bork P (2011) Interactive Tree Of Life v2: online annotation and display of phylogenetic trees made easy. Nucleic Acids Research 39, W475-W478.

Lyko F, Ramashoye BH, Jaenisch R (2000) DNA methylation in Drosophila melanogaster. Nature 408, 538-540.

Mastretta-Yanes A, Arrigo N, Alvarez N, et al. (2014) Restriction site-associated DNA sequencing, genotyping error estimation and de novo assembly optimization for population genetic inference. Molecular Ecology Resources 15, 28-41.

Miller MR, Dunham JP, Amores A, Cresko WA, Johnson EA (2007) Rapid and cost-effective polymorphism identification and genotyping using restriction site associated DNA (RAD) markers. Genome Research 17, 240-248.

Peterson BK, Weber JN, Kay EH, Fisher HS, Hoekstra HE (2012) Double digest RADseq: An inexpensive method for de novo SNP discovery and genotyping in model and non-model species. PLoS One 7, e37135.

Rambach A, Tiollais P (1974) Bacteriophage having EcoRI endonucleases sites only in the nonessential sites of the genome. Proceedings of the National Academy of Sciences of the United States of America 71, 3927-3930.

Reitzel AM, Herrera S, Layden MJ, Martindale MQ, Shank TM (2013) Going where traditional markers have not gone before: utility of and promise for RAD sequencing in marine invertebrate phylogeography and population genomics. Molecular Ecology 22, 2953-2970.

Rocha EPC, Danchin A, Viari A (2001) Evolutionary role of restriction/modification systems as revealed by comparative genome analysis. Genome Research 11, 946-958.

Scaglione D, Acquadro A, Portis E, et al. (2012) RAD tag sequencing as a source of SNP markers in Cynara cardunculus L. BMC Genomics 13, 3.

Singh GB (2009) Stochastic Models for Biological Patterns. In: Bioinformatics for Systems Biology (ed. Krawetz S), pp. 151-162. Springer, New York.

Šmarda P, Bureš P, Šmerda J, Horová L (2011) Measurements of genomic GC content in plant genomes with flow cytometry: a test for reliability. New Phytologist 193, 513-521.

Toonen RJ, Puritz JB, Forsman ZH, et al. (2013) ezRAD: A simplified method for genomic genotyping in non-model organisms. PeerJ 1, e203.

Vinogradov A (1994) Measurement by flow cytometry of genomic AT/GC ratio and genome size. Cytometry 16, 34-40.

Vinogradov A (1998) Genome size and GC-percent in vertebrates as determined by flow cytometry: The triangular relationship. Cytometry 31, 100-109.

Wagner CE, Keller I, Wittwer S, et al. (2012) Genome-wide RAD sequence data provide unprecedented resolution of species boundaries and relationships in the Lake Victoria cichlid adaptive radiation. Molecular Ecology 22, 787-798.

Wang J, Wurm Y, Nipitwattanaphon M, et al. (2013) A Y-like social chromosome causes alternative colony organization in fire ants. Nature 493, 664-668.

Weber JN, Peterson BK, Hoekstra HE (2013) Discrete genetic modules are responsible for complex burrow evolution in Peromyscus mice. Nature 493, 402-405. 
White TA, Perkins SE, Heckel G, Searle JB (2013) Adaptive evolution during an ongoing range expansion: the invasive bank vole (Myodes glareolus) in Ireland. Molecular Ecology 22, 29712985.

Yoder JA, Walsh CP, Bestor TH (1997) Cytosine methylation and the ecology of intragenomic parasites. Trends in Genetics 13, 335-340.

\section{SUPPORTING INFORMATION}


Table S1. Genome assemblies included in this study. Note that web addresses to individual assembly files, and the assembly files themselves, were current as of December 2012 and may have changed.

\section{ID}

Species

Acanthamoeba castellan

Aciric Acidomyces richmondensis

Acralc Acremonium alcalophilum

Acrech Acromyrmex echinatior

Acrdig Acropora digitifera

Acypis Acyrthosiphon pisum

Aedaeg Aedes aegypti

Agabis Agaricus bisporus

Ailmel Ailuropoda melanoleuca

Ajecap Ajellomyces capsulatus

Alamos Alatina moseri

Albcan Albugo candida

Allmis Alligator mississippiensis

Allmac Allomyces macrogynus

Altarb Alternaria arborescens

Amamus Amanita muscaria

Ampque Amphimedon queenslandica

Annalg Anncaliia algerae

Anocar Anolis carolinensis

Anogam Anopheles gambiae

Apimon Apiospora montagnei

Apimel Apis mellifera

Aplcal Aplysia californica

Aratha Arabidopsis thaliana

Artoli Arthrobotrys oligospora

Artben Arthroderma benhamiae

Ascsuu Ascaris suum

Ascrub Ascoidea rubescens

Ascapi Ascosphaera apis

Aspfum Aspergillus fumigatus

Ast sp Asterochloris $s p$

Attcep Atta cephalotes

Aurlim Aurantiochytrium limacinum

Aurpul Aureobasidium pullulans

Aurano Aureococcus anophagefferens

Aurdel Auricularia delicata

Babbov Babesia bovis

Babino Babjeviella inositovora

\section{Genome Assembly}

http://www.ncbi.nlm.nih.gov/Traces/wgs/?download=AEYA01.fasta.gz

http://www.ncbi.nlm.nih.gov/Traces/wgs/?download=AFQZ01.fasta.gz

http://genome.jgi-psf.org/Aciri1_iso/download/Aciri1_iso_AssemblyScaffolds.fasta.gz

http://genome.jgi-psf.org/Acral2/download/Acral2_AssemblyScaffolds.fasta.gz

http://www.ncbi.nlm.nih.gov/Traces/wgs/?download=AEVX01.fasta.gz

http://www.ncbi.nlm.nih.gov/Traces/wgs/?download=BACK01.fasta.gz

http://www.ncbi.nlm.nih.gov/Traces/wgs/?download=ABLF02.fasta.gz

http://www.ncbi.nlm.nih.gov/Traces/wgs/?download=AAGE02.fasta.gz

http://www.ncbi.nlm.nih.gov/Traces/wgs/?download=AEOK01.fasta.gz

http://www.ncbi.nlm.nih.gov/Traces/wgs/?download=ACTA01.fasta.gz

http://www.ncbi.nlm.nih.gov/Traces/wgs/?download=AAJI01.fasta.gz

http://www.ncbi.nlm.nih.gov/Traces/wgs/?download=AHZO01.fasta.gz

http://www.ncbi.nlm.nih.gov/Traces/wgs/?download=CAJG01.fasta.gz

http://www.ncbi.nlm.nih.gov/Traces/wgs/?download=AKHW01.fasta.gz

http://www.ncbi.nlm.nih.gov/Traces/wgs/?download=ACDU01.fasta.gz

http://www.ncbi.nlm.nih.gov/Traces/wgs/?download=AllC01.fasta.gz

http://genome.jgi-psf.org/Amamu1/download/Amamu1_AssemblyScaffolds.fasta.gz

ftp://ftp.ncbi.nlm.nih.gov/genbank/genomes/Eukaryotes/invertebrates/Amphimedon_queenslandica/v1.0/l

http://www.ncbi.nlm.nih.gov/Traces/wgs/?download=CAIR01.fasta.gz

http://www.ncbi.nlm.nih.gov/Traces/wgs/?download=AAWZ02.fasta.gz

http://www.ncbi.nlm.nih.gov/Traces/wgs/?download=AAAB01.fasta.gz

http://genome.jgi-psf.org/Apimo1/download/Apimo1_AssemblyScaffolds.fasta.gz

http://www.ncbi.nlm.nih.gov/Traces/wgs/?download=AADG06.fasta.gz

http://www.ncbi.nlm.nih.gov/Traces/wgs/?download=AASC02.fasta.gz

http://www.ncbi.nlm.nih.gov/Traces/wgs/?download=AFNA01.fasta.gz

http://www.ncbi.nlm.nih.gov/Traces/wgs/?download=ADOT01.fasta.gz

http://www.ncbi.nlm.nih.gov/Traces/wgs/?download=ABSU01.fasta.gz

http://www.ncbi.nlm.nih.gov/Traces/wgs/?download=AMPH01.fasta.gz

http://genome.jgi-psf.org/Ascru1/download/Ascru1_AssemblyScaffolds.fasta.gz

http://www.ncbi.nlm.nih.gov/Traces/wgs/?download=AARE01.fasta.gz

http://www.ncbi.nlm.nih.gov/Traces/wgs/?download=AAHF01.fasta.gz

http://genome.jgi-psf.org/Astpho1/download/Astpho1_genomic_scaffolds.fasta.gz

http://www.ncbi.nlm.nih.gov/Traces/wgs/?download=ADTU01.fasta.gz

http://genome.jgi-psf.org/Aurli1/download/Aurli1_AssemblyScaffolds.fasta.gz

http://www.ncbi.nlm.nih.gov/Traces/wgs/?download=AMCU01.fasta.gz

http://www.ncbi.nlm.nih.gov/Traces/wgs/?download=ACJI01.fasta.gz

http://www.ncbi.nlm.nih.gov/Traces/wgs/?download=AFVO01.fasta.gz

http://www.ncbi.nlm.nih.gov/Traces/wgs/?download=AAXT01.fasta.gz

http://genome.jgi-psf.org/Babin1/download/Babin1_AssemblyScaffolds.fasta.gz 


\begin{tabular}{|c|c|c|}
\hline Batden & Batrachochytrium dendrobatidis & http://www.ncbi.nlm.nih.gov/Traces/wgs/?download=ADAR01.fasta.gz \\
\hline Baucom & Baudoinia compniacensis & http://genome.jgi-psf.org/Bauco1/download/Bauco1_AssemblyScaffolds.fasta.gz \\
\hline Beabas & Beauveria bassiana & http://www.ncbi.nlm.nih.gov/Traces/wgs/?download=ADAH01.fasta.gz \\
\hline Betnan & Betula nana & http://www.ncbi.nlm.nih.gov/Traces/wgs/?download=CAOK01.fasta.gz \\
\hline Bignat & Bigelowiella natans & http://www.ncbi.nlm.nih.gov/Traces/wgs/?download=ADNK01.fasta.gz \\
\hline Bjeadu & Bjerkandera adusta & http://genome.jgi-psf.org/Bjead1_1/download/Bjead1_1_AssemblyScaffolds.fasta.gz \\
\hline Blahom & Blastocystis hominis & http://www.ncbi.nlm.nih.gov/Traces/wgs/?download=CABX01.fasta.gz \\
\hline Blugra & Blumeria graminis & http://www.ncbi.nlm.nih.gov/Traces/wgs/?download=ABSB02.fasta.gz \\
\hline Bomter & Bombus terrestris & http://www.ncbi.nlm.nih.gov/Traces/wgs/?download=AELG01.fasta.gz \\
\hline Bommor & Bombyx mori & http://www.ncbi.nlm.nih.gov/Traces/wgs/?download=BABH01.fasta.gz \\
\hline Bostau & Bos taurus & http://www.ncbi.nlm.nih.gov/Traces/wgs/?download=AAFC03.fasta.gz \\
\hline Botbot & Botryobasidium botryosum & http://genome.jgi-psf.org/Botbo1/download/Botbo1_AssemblyScaffolds.fasta.gz \\
\hline Botfuc & Botryotinia fuckeliana & http://www.ncbi.nlm.nih.gov/Traces/wgs/?download=ALOC01.fasta.gz \\
\hline Bradis & Brachypodium distachyon & http://www.ncbi.nlm.nih.gov/Traces/wgs/?download=ADDN01.fasta.gz \\
\hline Braflo & Branchiostoma floridae & http://www.ncbi.nlm.nih.gov/Traces/wgs/?download=ABEP02.fasta.gz \\
\hline Brarap & Brassica rapa & http://www.ncbi.nlm.nih.gov/Traces/wgs/?download=AENI01.fasta.gz \\
\hline Brumal & Brugia malayi & http://www.ncbi.nlm.nih.gov/Traces/wgs/?download=AAQA01.fasta.gz \\
\hline Burxyl & Bursaphelenchus xylophilus & http://www.ncbi.nlm.nih.gov/Traces/wgs/?download=CADV01.fasta.gz \\
\hline Caeele & Caenorhabditis elegans & $\mathrm{ftp}: / /$ ttp.wormbase.org/pub/wormbase/genomes/c_elegans/sequences/dna/c_elegans.WS201.dna.fa.gz \\
\hline Cajcaj & Cajanus cajan & http://www.ncbi.nlm.nih.gov/Traces/wgs/?download=AFSP01.fasta.gz \\
\hline Caljac & Callithrix jacchus & http://www.ncbi.nlm.nih.gov/Traces/wgs/?download=ACFV01.fasta.gz \\
\hline Calmil & Callorhinchus milii & http://www.ncbi.nlm.nih.gov/Traces/wgs/?download=AAVX01.fasta.gz \\
\hline Camfer & Camelus ferus & http://www.ncbi.nlm.nih.gov/Traces/wgs/?download=AGVR01.fasta.gz \\
\hline Camflo & Camponotus floridanus & http://www.ncbi.nlm.nih.gov/Traces/wgs/?download=AEAB01.fasta.gz \\
\hline Canalb & Candida albicans & http://www.ncbi.nlm.nih.gov/Traces/wgs/?download=AAFO01.fasta.gz \\
\hline Canlup & Canis lupus & http://www.ncbi.nlm.nih.gov/Traces/wgs/?download=AAEX03.fasta.gz \\
\hline Cansat & Cannabis sativa & http://www.ncbi.nlm.nih.gov/Traces/wgs/?download=AGQN01.fasta.gz \\
\hline Captel & Capitella teleta & ftp://ftp.jgi-psf.org/pub/JGI_data/Capitella/v1.0/Capitella_spl.fasta.gz \\
\hline Capowc & Capsaspora owczarzaki & http://www.ncbi.nlm.nih.gov/Traces/wgs/?download=ACFS01.fasta.gz \\
\hline Carpap & Carica papaya & http://www.ncbi.nlm.nih.gov/Traces/wgs/?download=ABIM01.fasta.gz \\
\hline Catang & Catenaria anguillulae & http://genome.jgi-psf.org/Catan1/download/Catan1_AssemblyScaffolds.fasta.gz \\
\hline Cavpor & Cavia porcellus & http://www.ncbi.nlm.nih.gov/Traces/wgs/?download=AAKN02.fasta.gz \\
\hline Cersim & Ceratotherium simum & http://www.ncbi.nlm.nih.gov/Traces/wgs/?download=AKZM01.fasta.gz \\
\hline Cerzea & Cercospora zeaemaydis & http://genome.jgi-psf.org/Cerzm1/download/Cerzm1_AssemblyScaffolds.fasta.gz \\
\hline Cersub & Ceriporiopsis subvermispora & http://www.ncbi.nlm.nih.gov/Traces/wgs/?download=AEOV01.fasta.gz \\
\hline Chathe & Chaetomium thermophilum & http://www.ncbi.nlm.nih.gov/Traces/wgs/?download=ADUW01.fasta.gz \\
\hline Chilan & Chinchilla lanigera & http://www.ncbi.nlm.nih.gov/Traces/wgs/?download=AGCD01.fasta.gz \\
\hline Chlrei & Chlamydomonas reinhardtii & http://www.ncbi.nlm.nih.gov/Traces/wgs/?download=ABCN01.fasta.gz \\
\hline Chlvar & Chlorella variabilis & http://www.ncbi.nlm.nih.gov/Traces/wgs/?download=ADIC01.fasta.gz \\
\hline Chohof & Choloepus hoffmanni & http://www.ncbi.nlm.nih.gov/Traces/wgs/?download=ABVD01.fasta.gz \\
\hline Chrpic & Chrysemys picta bellii & http://www.ncbi.nlm.nih.gov/Traces/wgs/?download=AHGY01.fasta.gz \\
\hline Chrasi & Chrysochloris asiatica & http://www.ncbi.nlm.nih.gov/Traces/wgs/?download=AMDV01.fasta.gz \\
\hline Cioint & Ciona intestinalis & http://www.ncbi.nlm.nih.gov/Traces/wgs/?download=AABS01.fasta.gz \\
\hline
\end{tabular}




\begin{tabular}{|c|c|c|}
\hline Citlan & Citrullus lanatus & http://www.ncbi.nlm.nih.gov/Traces/wgs/?download=AGCB01.fasta.gz \\
\hline Citsin & Citrus sinensis & http://www.ncbi.nlm.nih.gov/Traces/wgs/?download=AJPS01.fasta.gz \\
\hline Clagra & Cladonia grayi & http://genome.jgi-psf.org/Clagr2/download/Clagr2_genomic_scaffolds.fasta.gz \\
\hline Clasph & Cladosporium sphaerospermum & http://www.ncbi.nlm.nih.gov/Traces/wgs/?download=AllA01.fasta.gz \\
\hline Clafus & Claviceps fusiformis & http://www.ncbi.nlm.nih.gov/Traces/wgs/?download=AFRA01.fasta.gz \\
\hline Clalus & Clavispora lusitaniae & http://www.ncbi.nlm.nih.gov/Traces/wgs/?download=AAFT01.fasta.gz \\
\hline Closin & Clonorchis sinensis & http://www.ncbi.nlm.nih.gov/Traces/wgs/?download=BADR02.fasta.gz \\
\hline Cocpos & Coccidioides posadasii & http://www.ncbi.nlm.nih.gov/Traces/wgs/?download=ACFW01.fasta.gz \\
\hline Cocsub & Coccomyxa subellipsoidea & http://www.ncbi.nlm.nih.gov/Traces/wgs/?download=AGSI01.fasta.gz \\
\hline Coclun & Cochliobolus lunatus & http://genome.jgi-psf.org/Coclu2/download/Coclu2_AssemblyScaffolds.fasta.gz \\
\hline Coerev & Coemansia reversa & http://genome.jgi-psf.org/Coere1/download/Coere1_AssemblyScaffolds.fasta.gz \\
\hline Colglo & Colletotrichum gloeosporioides & http://www.ncbi.nlm.nih.gov/Traces/wgs/?download=ANPB01.fasta.gz \\
\hline Concri & Condylura cristata & http://www.ncbi.nlm.nih.gov/Traces/wgs/?download=AJFV01.fasta.gz \\
\hline Concor & Conidiobolus coronatus & http://genome.jgi-psf.org/Conco1/download/Conco1_AssemblyScaffolds.fasta.gz \\
\hline Conput & Coniophora puteana & http://www.ncbi.nlm.nih.gov/Traces/wgs/?download=AEIT01.fasta.gz \\
\hline Conapo & Coniosporium apollinis & http://www.ncbi.nlm.nih.gov/Traces/wgs/?download=AJKL01.fasta.gz \\
\hline Copcin & Coprinopsis cinerea & http://www.ncbi.nlm.nih.gov/Traces/wgs/?download=AACS02.fasta.gz \\
\hline Cormil & Cordyceps militaris & http://www.ncbi.nlm.nih.gov/Traces/wgs/?download=AEVU01.fasta.gz \\
\hline Cragig & Crassostrea gigas & http://www.ncbi.nlm.nih.gov/Traces/wgs/?download=AFT101.fasta.gz \\
\hline Crigri & Cricetulus griseus & http://www.ncbi.nlm.nih.gov/Traces/wgs/?download=AFTD01.fasta.gz \\
\hline Croque & Cronartium quercuum & http://genome.jgi-psf.org/Croqu1/download/Croqu1_AssemblyScaffolds.fasta.gz \\
\hline Crypa2 & Cryphonectria parasitica & http://genome.jgi-psf.org/Crypa2/download/Cryphonectria_parasiticav2.nuclearAssembly.unmasked.gz \\
\hline Crygat & Cryptococcus gattii & http://www.ncbi.nlm.nih.gov/Traces/wgs/?download=AAFP01.fasta.gz \\
\hline Crypa1 & Cryptosporidium parvum & http://www.ncbi.nlm.nih.gov/Traces/wgs/?download=AAEE01.fasta.gz \\
\hline Cucsat & Cucumis sativus & http://www.ncbi.nlm.nih.gov/Traces/wgs/?download=ACHR01.fasta.gz \\
\hline Culqui & Culex quinquefasciatus & http://www.ncbi.nlm.nih.gov/Traces/wgs/?download=AAAB01.fasta.gz \\
\hline Cyamer & Cyanidioschyzon merolae & http://www.ebi.ac.uk/ena/data/view/AP006483.2,AP006492.2,AP006493.2,AP006494.2,AP006495.2,APC \\
\hline Cybjad & Cyberlindnera jadinii & http://www.ncbi.nlm.nih.gov/Traces/wgs/?download=BAEL01.fasta.gz \\
\hline Dac_sp & Dacryopinax sp & http://www.ncbi.nlm.nih.gov/Traces/wgs/?download=AEUS01.fasta.gz \\
\hline Dalesc & Daldinia eschscholzii & http://www.ncbi.nlm.nih.gov/Traces/wgs/?download=AllD01.fasta.gz \\
\hline Danple & Danaus plexippus & ftp://ttp.ensemblgenomes.org/pub/metazoa/release-16/fasta/danaus_plexippus/dna/Danaus_plexippus.D \\
\hline Danrer & Danio rerio & http://www.ncbi.nlm.nih.gov/Traces/wgs/?download=CABZ01.fasta.gz \\
\hline Dappul & Daphnia pulex & http://www.ncbi.nlm.nih.gov/Traces/wgs/?download=ACJG01.fasta.gz \\
\hline Dasnov & Dasypus novemcinctus & http://www.ncbi.nlm.nih.gov/Traces/wgs/?download=AAGV03.fasta.gz \\
\hline Daumad & Daubentonia madagascariensis & http://www.ncbi.nlm.nih.gov/Traces/wgs/?download=AGTM01.fasta.gz \\
\hline Debhan & Debaryomyces hansenii & http://www.ncbi.nlm.nih.gov/Traces/wgs/?download=AHBE01.fasta.gz \\
\hline Dekbru & Dekkera bruxellensis & http://www.ncbi.nlm.nih.gov/Traces/wgs/?download=AHIQ01.fasta.gz \\
\hline Diclab & Dicentrarchus labrax & http://www.ncbi.nlm.nih.gov/Traces/wgs/?download=CABK01.fasta.gz \\
\hline Dicsqu & Dichomitus squalens & http://www.ncbi.nlm.nih.gov/Traces/wgs/?download=AEID01.fasta.gz \\
\hline Dicdis & Dictyostelium discoideum & http://www.ncbi.nlm.nih.gov/Traces/wgs/?download=AAFI02.fasta.gz \\
\hline Didexi & Didymella exigua & http://genome.jgi-psf.org/Didex1/download/Didex1_AssemblyScaffolds.fasta.gz \\
\hline Dipord & Dipodomys ordii & http://www.ncbi.nlm.nih.gov/Traces/wgs/?download=ABRO01.fasta.gz \\
\hline Dromel & Drosophila melanogaster & http://www.ncbi.nlm.nih.gov/Traces/wgs/?download=AABU01.fasta.gz \\
\hline
\end{tabular}




\begin{tabular}{|c|c|c|}
\hline Echtel & Echinops telfairi & http://www.ncbi.nlm.nih.gov/Traces/wgs/?download=AAIY02.fasta.gz \\
\hline Ectsil & Ectocarpus siliculosus & http://www.ncbi.nlm.nih.gov/Traces/wgs/?download=CABU01.fasta.gz \\
\hline Edhaed & Edhazardia aedis & http://www.ncbi.nlm.nih.gov/Traces/wgs/?download=AFBI02.fasta.gz \\
\hline Eleedw & Elephantulus edwardii & http://www.ncbi.nlm.nih.gov/Traces/wgs/?download=AMGZ01.fasta.gz \\
\hline Emihux & Emiliania huxleyi & http://genome.jgi-psf.org/Emihu1/download/Emihu1_scaffolds.fasta.gz \\
\hline Enccun & Encephalitozoon cuniculi & http://www.ncbi.nlm.nih.gov/Traces/wgs/?download=AEWD01.fasta.gz \\
\hline Enthis & Entamoeba histolytica & http://www.ncbi.nlm.nih.gov/Traces/wgs/?download=AAFB02.fasta.gz \\
\hline Entbie & Enterocytozoon bieneusi & http://www.ncbi.nlm.nih.gov/Traces/wgs/?download=ABGB01.fasta.gz \\
\hline Epityp & Epichloe typhina & http://www.ncbi.nlm.nih.gov/Traces/wgs/?download=AMDI01.fasta.gz \\
\hline Eptfus & Eptesicus fuscus & http://www.ncbi.nlm.nih.gov/Traces/wgs/?download=ALEH01.fasta.gz \\
\hline Equcab & Equus caballus & http://www.ncbi.nlm.nih.gov/Traces/wgs/?download=AAWR02.fasta.gz \\
\hline Erieur & Erinaceus europaeus & http://www.ncbi.nlm.nih.gov/Traces/wgs/?download=AMDU01.fasta.gz \\
\hline Erypis & Erysiphe pisi & http://www.ncbi.nlm.nih.gov/Traces/wgs/?download=CACM01.fasta.gz \\
\hline Euccam & Eucalyptus camaldulensis & http://www.ncbi.nlm.nih.gov/Traces/wgs/?download=BADO01.fasta.gz \\
\hline Eurher & Eurotium herbariorum & http://genome.jgi-psf.org/Eurhe1/download/Eurhe1_AssemblyScaffolds.fasta.gz \\
\hline Eutpar & Eutrema parvulum & http://www.ncbi.nlm.nih.gov/Traces/wgs/?download=AFAN01.fasta.gz \\
\hline Exoder & Exophiala dermatitidis & http://www.ncbi.nlm.nih.gov/Traces/wgs/?download=AFPA01.fasta.gz \\
\hline Felcat & Felis catus & http://www.ncbi.nlm.nih.gov/Traces/wgs/?download=AANG02.fasta.gz \\
\hline Fibrad & Fibroporia radiculosa & http://www.ncbi.nlm.nih.gov/Traces/wgs/?download=CAGC01.fasta.gz \\
\hline Ficalb & Ficedula albicollis & http://www.ncbi.nlm.nih.gov/Traces/wgs/?download=AGTO01.fasta.gz \\
\hline Fommed & Fomitiporia mediterranea & http://www.ncbi.nlm.nih.gov/Traces/wgs/?download=AEJJ01.fasta.gz \\
\hline Fompin & Fomitopsis pinicola & http://genome.jgi-psf.org/Fompi3/download/Fompi3_AssemblyScaffolds.fasta.gz \\
\hline Fraves & Fragaria vesca & http://www.ncbi.nlm.nih.gov/Traces/wgs/?download=AEMH01.fasta.gz \\
\hline Fracyl & Fragilariopsis cylindrus & http://genome.jgi-psf.org/Fracy1/download/portalData/Fracy1_assembly_scaffolds.fasta.gz \\
\hline Fusoxy & Fusarium oxysporum & http://www.ncbi.nlm.nih.gov/Traces/wgs/?download=AAXH01.fasta.gz \\
\hline Gadmor & Gadus morhua & http://www.ncbi.nlm.nih.gov/Traces/wgs/?download=CAEA01.fasta.gz \\
\hline Gaegra & Gaeumannomyces graminis & http://www.ncbi.nlm.nih.gov/Traces/wgs/?download=ADBI01.fasta.gz \\
\hline Galmar & Galerina marginata & http://genome.jgi-psf.org/Galma1/download/Galma1_AssemblyScaffolds.fasta.gz \\
\hline Galgal & Gallus gallus & http://www.ncbi.nlm.nih.gov/Traces/wgs/?download=AADN03.fasta.gz \\
\hline Ganluc & Ganoderma lucidum & http://www.ncbi.nlm.nih.gov/Traces/wgs/?download=AGAX01.fasta.gz \\
\hline Gasacu & Gasterosteus aculeatus & http://www.ncbi.nlm.nih.gov/Traces/wgs/?download=AANH01.fasta.gz \\
\hline Geodes & Geomyces destructans & http://www.ncbi.nlm.nih.gov/Traces/wgs/?download=AEFC01.fasta.gz \\
\hline Geofor & Geospiza fortis & http://www.ncbi.nlm.nih.gov/Traces/wgs/?download=AKZB01.fasta.gz \\
\hline Gialam & Giardia lamblia & http://www.ncbi.nlm.nih.gov/Traces/wgs/?download=AACB02.fasta.gz \\
\hline Gibmon & Gibberella moniliformis & http://www.ncbi.nlm.nih.gov/Traces/wgs/?download=AAIM02.fasta.gz \\
\hline Glaloz & Glarea lozoyensis & http://www.ncbi.nlm.nih.gov/Traces/wgs/?download=AGUE01.fasta.gz \\
\hline Glotra & Gloeophyllum trabeum & http://genome.jgi-psf.org/Glotr1_1/download/Glotr1_1_AssemblyScaffolds.fasta.gz \\
\hline Glogra & Glomerella graminicola & http://www.ncbi.nlm.nih.gov/Traces/wgs/?download=ACOD01.fasta.gz \\
\hline Glymax & Glycine $\max$ & http://www.ncbi.nlm.nih.gov/Traces/wgs/?download=ACUP01.fasta.gz \\
\hline Gonpro & Gonapodya prolifera & http://genome.jgi-psf.org/Ganpr1/download/Ganpr1_AssemblyScaffolds.fasta.gz \\
\hline Gorgor & Gorilla gorilla & http://www.ncbi.nlm.nih.gov/Traces/wgs/?download=CABD02.fasta.gz \\
\hline Gosrai & Gossypium raimondii & http://www.ncbi.nlm.nih.gov/Traces/wgs/?download=ALYE01.fasta.gz \\
\hline Grocla & Grosmannia clavigera & http://www.ncbi.nlm.nih.gov/Traces/wgs/?download=ACXQ02.fasta.gz \\
\hline
\end{tabular}




\begin{tabular}{|c|c|c|}
\hline Guithe & Guillardia theta & http://www.ncbi.nlm.nih.gov/Traces/wgs/?download=AEIE01.fasta.gz \\
\hline Gymlux & Gymnopus luxurians & http://genome.jgi-psf.org/Gymlu1/download/Gymlu1_AssemblyScaffolds.fasta.gz \\
\hline Hamtva & Hamiltosporidium tvaerminnensis & http://www.ncbi.nlm.nih.gov/Traces/wgs/?download=ACSZ01.fasta.gz \\
\hline Hamham & Hammondia hammondi & http://www.ncbi.nlm.nih.gov/Traces/wgs/?download=AHJH01.fasta.gz \\
\hline Hanval & Hanseniaspora valbyensis & http://genome.jgi-psf.org/Hanva1_1/download/Hanva1_1_AssemblyScaffolds.fasta.gz \\
\hline Hapbur & Haplochromis burtoni & http://www.ncbi.nlm.nih.gov/Traces/wgs/?download=AFNZ01.fasta.gz \\
\hline Harsal & Harpegnathos saltator & http://www.ncbi.nlm.nih.gov/Traces/wgs/?download=AEAC01.fasta.gz \\
\hline Hebcyl & Hebeloma cylindrosporum & http://genome.jgi-psf.org/Hebcy2/download/Hebcy2_AssemblyScaffolds.fasta.gz \\
\hline Helmel & Heliconius melpomene & http://www.ncbi.nlm.nih.gov/Traces/wgs/?download=CAEZ01.fasta.gz \\
\hline Helrob & Helobdella robusta & ftp://ftp.jgi-psf.org/pub/JGI_data/Helobdella_robusta/v1.0/Helobdella_robusta.fasta.gz \\
\hline Hetirr & Heterobasidion irregulare & http://www.ncbi.nlm.nih.gov/Traces/wgs/?download=AEOJ01.fasta.gz \\
\hline Hetgla & Heterocephalus glaber & http://www.ncbi.nlm.nih.gov/Traces/wgs/?download=AHKG01.fasta.gz \\
\hline Hetgly & Heterodera glycines & http://www.ncbi.nlm.nih.gov/Traces/wgs/?download=ABLA01.fasta.gz \\
\hline Hetbac & Heterorhabditis bacteriophora & http://www.ncbi.nlm.nih.gov/Traces/wgs/?download=ACKM01.fasta.gz \\
\hline Homsap & Homo sapiens & http://www.ncbi.nlm.nih.gov/Traces/wgs/?download=AMYH01.fasta.gz \\
\hline Hompol & Homoloaphlyctis polyrhiza & http://www.ncbi.nlm.nih.gov/Traces/wgs/?download=AFSM01.fasta.gz \\
\hline Horvul & Hordeum vulgare & http://www.ncbi.nlm.nih.gov/Traces/wgs/?download=CAJW01.fasta.gz \\
\hline Hyaara & Hyaloperonospora arabidopsidis & http://www.ncbi.nlm.nih.gov/Traces/wgs/?download=ABWE02.fasta.gz \\
\hline Hydpin & Hydnomerulius pinastri & http://genome.jgi-psf.org/Hydpi2/download/Hydpi2_AssemblyScaffolds.fasta.gz \\
\hline Hydmag & Hydra magnipapillata & http://www.ncbi.nlm.nih.gov/Traces/wgs/?download=ABRM01.fasta.gz \\
\hline Hypcat & Hyphochytrium catenoides & http://www.ncbi.nlm.nih.gov/Traces/wgs/?download=CAFC02.fasta.gz \\
\hline Hypsub & Hypholoma sublateritium & http://genome.jgi-psf.org/Hypsu1/download/Hypsu1_AssemblyScaffolds.fasta.gz \\
\hline Hypbur & Hyphopichia burtonii & http://genome.jgi-psf.org/Hypbu1/download/Hypbu1_AssemblyScaffolds.fasta.gz \\
\hline Hyp_sp & Hypoxylon sp & http://genome.jgi-psf.org/HypCO275_1/download/HypCO275_1_AssemblyScaffolds.fasta.gz \\
\hline Ichmul & Ichthyophthirius multifiliis & http://www.ncbi.nlm.nih.gov/Traces/wgs/?download=AEDN01.fasta.gz \\
\hline Ixosca & Ixodes scapularis & http://www.ncbi.nlm.nih.gov/Traces/wgs/?download=ABJB01.fasta.gz \\
\hline Jaaarg & Jaapia argillacea & http://genome.jgi-psf.org/Jaaar1/download/Jaaar1_AssemblyScaffolds.fasta.gz \\
\hline Jacjac & Jaculus jaculus & http://www.ncbi.nlm.nih.gov/Traces/wgs/?download=AKZC01.fasta.gz \\
\hline Jatcur & Jatropha curcas & http://www.ncbi.nlm.nih.gov/Traces/wgs/?download=BABX01.fasta.gz \\
\hline Klumar & Kluyveromyces marxianus & http://www.ncbi.nlm.nih.gov/Traces/wgs/?download=AKFM01.fasta.gz \\
\hline Kompas & Komagataella pastoris & http://www.ncbi.nlm.nih.gov/Traces/wgs/?download=CABH01.fasta.gz \\
\hline Labfue & Labeotropheus fuelleborni & http://www.ncbi.nlm.nih.gov/Traces/wgs/?download=ABPK01.fasta.gz \\
\hline Lacbic & Laccaria bicolor & http://www.ncbi.nlm.nih.gov/Traces/wgs/?download=ABFE01.fasta.gz \\
\hline Lacklu & Lachancea kluyveri & http://www.ncbi.nlm.nih.gov/Traces/wgs/?download=AACE03.fasta.gz \\
\hline Lacsat & Lactuca sativa & http://www.ncbi.nlm.nih.gov/Traces/wgs/?download=AFSA01.fasta.gz \\
\hline Latcha & Latimeria chalumnae & http://www.ncbi.nlm.nih.gov/Traces/wgs/?download=AFYH01.fasta.gz \\
\hline Leeper & Leersia perrieri & http://www.ncbi.nlm.nih.gov/Traces/wgs/?download=ALNV01.fasta.gz \\
\hline Leibra & Leishmania braziliensis & http://www.ncbi.nlm.nih.gov/Traces/wgs/?download=CADA01.fasta.gz \\
\hline Lepsal & Lepeophtheirus salmonis & http://www.ncbi.nlm.nih.gov/Traces/wgs/?download=ADND02.fasta.gz \\
\hline Lepocu & Lepisosteus oculatus & http://www.ncbi.nlm.nih.gov/Traces/wgs/?download=AHAT01.fasta.gz \\
\hline Leueri & Leucoraja erinacea & http://www.ncbi.nlm.nih.gov/Traces/wgs/?download=AESE01.fasta.gz \\
\hline Linhum & Linepithema humile & http://www.ncbi.nlm.nih.gov/Traces/wgs/?download=ADOQ01.fasta.gz \\
\hline Linusi & Linum usitatissimum & http://www.ncbi.nlm.nih.gov/Traces/wgs/?download=AFSQ01.fasta.gz \\
\hline
\end{tabular}




\begin{tabular}{|c|c|c|}
\hline Lipsta & Lipomyces starkeyi & http://genome.jgi-psf.org/Lipst1_1/download/Lipst1_1_AssemblyScaffolds.fasta.gz \\
\hline Loaloa & Loa loa & http://www.ncbi.nlm.nih.gov/Traces/wgs/?download=ADBU02.fasta.gz \\
\hline Lodelo & Lodderomyces elongisporus & http://www.ncbi.nlm.nih.gov/Traces/wgs/?download=AAPO01.fasta.gz \\
\hline Lotgig & Lottia gigantea & ftp://ftp.jgi-psf.org/pub/JGI_data/Lottia_gigantea/v1.0/Lotgi1_assembly_scaffolds.fasta.gz \\
\hline Lotjap & Lotus japonicus & http://www.ncbi.nlm.nih.gov//races/wgs/?download=BABK01.fasta.gz \\
\hline Loxafr & Loxodonta africana & http://www.ncbi.nlm.nih.gov/Traces/wgs/?download=AAGU03.fasta.gz \\
\hline Lutlon & Lutzomyia longipalpis & http://www.ncbi.nlm.nih.gov/Traces/wgs/?download=AJWK01.fasta.gz \\
\hline Lytvar & Lytechinus variegatus & $\mathrm{ftp}: / /$ ttp.ncbi.nlm.nih.gov/genbank/genomes/Eukaryotes/invertebrates/Lytechinus_variegatus/Lvar_0.4/Pri \\
\hline Macfas & Macaca fascicularis & http://www.ncbi.nlm.nih.gov/Traces/wgs/?download=CAEC01.fasta.gz \\
\hline Macpha & Macrophomina phaseolina & http://www.ncbi.nlm.nih.gov/Traces/wgs/?download=AHHD01.fasta.gz \\
\hline Maceug & Macropus eugenii & http://www.ncbi.nlm.nih.gov/Traces/wgs/?download=ABQO02.fasta.gz \\
\hline Magory & Magnaporthe oryzae & http://www.ncbi.nlm.nih.gov/Traces/wgs/?download=AACU03.fasta.gz \\
\hline Malglo & Malassezia globosa & http://www.ncbi.nlm.nih.gov/Traces/wgs/?download=AAYY01.fasta.gz \\
\hline Maldom & Malus domestica & http://www.ncbi.nlm.nih.gov/Traces/wgs/?download=ACYM01.fasta.gz \\
\hline Mansex & Manduca sexta & http://www.ncbi.nlm.nih.gov/Traces/wgs/?download=AIXA01.fasta.gz \\
\hline Manesc & Manihot esculenta & ftp://ftp.jgi-psf.org/pub/compgen/phytozome/v9.0/Mesculenta/assembly/Mesculenta_147.fa.gz \\
\hline Marbru & Marssonina brunnea & http://www.ncbi.nlm.nih.gov/Traces/wgs/?download=AFXC01.fasta.gz \\
\hline Maydes & Mayetiola destructor & http://www.ncbi.nlm.nih.gov/Traces/wgs/?download=AEGA01.fasta.gz \\
\hline Mayzeb & Maylandia zebra & http://www.ncbi.nlm.nih.gov/Traces/wgs/?download=AHAT01.fasta.gz \\
\hline Mchcon & Mchenga conophoros & http://www.ncbi.nlm.nih.gov/Traces/wgs/?download=ABPJ01.fasta.gz \\
\hline Medtru & Medicago truncatula & ftp://ftp.jcvi.org/pub/data/m_truncatula/Mt3.5/Assembly/Mt3.5.2/MedtrA17_3.5.assemblies.fasta \\
\hline Megrot & Megachile rotundata & http://www.ncbi.nlm.nih.gov/Traces/wgs/?download=AAAB01.fasta.gz \\
\hline Mellar & Melampsora larici & http://www.ncbi.nlm.nih.gov/Traces/wgs/?download=AECX01.fasta.gz \\
\hline Mel_sp & Melanconium sp & http://genome.jgi-psf.org/Melsp1/download/Melsp1_AssemblyScaffolds.fasta.gz \\
\hline Melaur & Melanochromis auratus & http://www.ncbi.nlm.nih.gov/Traces/wgs/?download=ABPL01.fasta.gz \\
\hline Melgal & Meleagris gallopavo & http://www.ncbi.nlm.nih.gov/Traces/wgs/?download=ADDD01.fasta.gz \\
\hline Melinc & Meloidogyne incognita & http://www.ncbi.nlm.nih.gov/Traces/wgs/?download=CABB01.fasta.gz \\
\hline Melund & Melopsittacus undulatus & http://www.ncbi.nlm.nih.gov/Traces/wgs/?download=AGAl01.fasta.gz \\
\hline Menmol & Mengenilla moldrzyki & http://www.ncbi.nlm.nih.gov/Traces/wgs/?download=AGDA01.fasta.gz \\
\hline Metacr & Metarhizium acridum & http://www.ncbi.nlm.nih.gov/Traces/wgs/?download=ADNI01.fasta.gz \\
\hline Metocc & Metaseiulus occidentalis & http://www.ncbi.nlm.nih.gov/Traces/wgs/?download=AFFJ01.fasta.gz \\
\hline Metfru & Metschnikowia fructicola & http://www.ncbi.nlm.nih.gov/Traces/wgs/?download=ANFW01.fasta.gz \\
\hline Meygui & Meyerozyma guilliermondii & http://www.ncbi.nlm.nih.gov/Traces/wgs/?download=AAFM01.fasta.gz \\
\hline Micvio & Microbotryum violaceum & http://www.ncbi.nlm.nih.gov/Traces/wgs/?download=AEIJ01.fasta.gz \\
\hline Micpus & Micromonas pusilla & http://www.ncbi.nlm.nih.gov/Traces/wgs/?download=ACCP01.fasta.gz \\
\hline Micoch & Microtus ochrogaster & http://www.ncbi.nlm.nih.gov/Traces/wgs/?download=AHZW01.fasta.gz \\
\hline Mimgut & Mimulus guttatus & ftp://ftp.jgi-psf.org/pub/compgen/phytozome/v9.0/Mguttatus_v1.1/assembly/Mguttatus_140.fa.gz \\
\hline Mixosm & Mixia osmundae & http://www.ncbi.nlm.nih.gov/Traces/wgs/?download=BABT02.fasta.gz \\
\hline Mnelei & Mnemiopsis leidyi & ftp://ftp.ncbi.nlm.nih.gov/genbank/genomes/Eukaryotes/invertebrates/Mnemiopsis_leidyi/MneLei_Aug201 \\
\hline Monper & Moniliophthora perniciosa & http://www.ncbi.nlm.nih.gov/Traces/wgs/?download=ABRE01.fasta.gz \\
\hline Mondom & Monodelphis domestica & http://www.ncbi.nlm.nih.gov/Traces/wgs/?download=AAFR03.fasta.gz \\
\hline Monbre & Monosiga brevicollis & http://www.ncbi.nlm.nih.gov/Traces/wgs/?download=ABFJ01.fasta.gz \\
\hline Morelo & Mortierella elongata & tp://genome.jgi-psf.org/Morel1/download/Morel1_AssemblyScaffolds.fasta.gz \\
\hline
\end{tabular}




\begin{tabular}{|c|c|c|}
\hline Muccir & Mucor circinelloides & http://genome.jgi-psf.org/Mucci2/download/Mucor_circinelloides_v2_scaffolds.fasta.gz \\
\hline Musmus & Mus musculus & http://www.ncbi.nlm.nih.gov/Traces/wgs/?download=AEKQ02.fasta.gz \\
\hline Musacu & Musa acuminata & http://www.ncbi.nlm.nih.gov/Traces/wgs/?download=CAIC01.fasta.gz \\
\hline Musput & Mustela putorius & http://www.ncbi.nlm.nih.gov/Traces/wgs/?download=AGTQ01.fasta.gz \\
\hline Mycthe & Myceliophthora thermophila & http://genome.jgi-psf.org/Spoth2/download/Spoth2_AssemblyScaffolds.fasta.gz \\
\hline Мyсрор & Mycosphaerella populicola & http://www.ncbi.nlm.nih.gov/Traces/wgs/?download=AIDU01.fasta.gz \\
\hline Myodav & Myotis davidii & http://www.ncbi.nlm.nih.gov/Traces/wgs/?download=ALWT01.fasta.gz \\
\hline Nadful & Nadsonia fulvescens & http://genome.jgi-psf.org/Nadfu1/download/Nadfu1_AssemblyScaffolds.fasta.gz \\
\hline Naegru & Naegleria gruberi & http://www.ncbi.nlm.nih.gov/Traces/wgs/?download=ACER01.fasta.gz \\
\hline Nangad & Nannochloropsis gaditana & http://www.ncbi.nlm.nih.gov/Traces/wgs/?download=AGNI01.fasta.gz \\
\hline Nasvit & Nasonia vitripennis & http://www.ncbi.nlm.nih.gov/Traces/wgs/?download=AAZX01.fasta.gz \\
\hline Naucas & Naumovozyma castellii & http://www.ncbi.nlm.nih.gov/Traces/wgs/?download=AACF01.fasta.gz \\
\hline Nechae & Nectria haematococca & http://www.ncbi.nlm.nih.gov/Traces/wgs/?download=ACJF01.fasta.gz \\
\hline Nempar & Nematocida parisii & http://www.ncbi.nlm.nih.gov/Traces/wgs/?download=AEFF02.fasta.gz \\
\hline Nemvec & Nematostella vectensis & http://genome.jgi.doe.gov/Nemve1/download/Nemve1.fasta.gz \\
\hline Neobri & Neolamprologus brichardi & http://www.ncbi.nlm.nih.gov/Traces/wgs/?download=AFNY01.fasta.gz \\
\hline Neofis & Neosartorya fischeri & http://www.ncbi.nlm.nih.gov/Traces/wgs/?download=AAKE03.fasta.gz \\
\hline Neogan & Neotyphodium gansuense & http://www.ncbi.nlm.nih.gov/Traces/wgs/?download=AMDK01.fasta.gz \\
\hline Neucra & Neurospora crassa & http://www.ncbi.nlm.nih.gov/Traces/wgs/?download=AABX02.fasta.gz \\
\hline Nomleu & Nomascus leucogenys & http://www.ncbi.nlm.nih.gov/Traces/wgs/?download=ADFV01.fasta.gz \\
\hline Noscer & Nosema ceranae & http://www.ncbi.nlm.nih.gov/Traces/wgs/?download=ACOL01.fasta.gz \\
\hline Ochpri & Ochotona princeps & http://www.ncbi.nlm.nih.gov/Traces/wgs/?download=ALIT01.fasta.gz \\
\hline Octdeg & Octodon degus & http://www.ncbi.nlm.nih.gov/Traces/wgs/?download=AJSA01.fasta.gz \\
\hline Ogapar & Ogataea parapolymorpha & http://www.ncbi.nlm.nih.gov/Traces/wgs/?download=AEOI01.fasta.gz \\
\hline Oidmai & Oidiodendron maius & http://genome.jgi-psf.org/Oidma1/download/Oidma1_AssemblyScaffolds.fasta.gz \\
\hline Oikdio & Oikopleura dioica & http://www.ncbi.nlm.nih.gov/Traces/wgs/?download=CABV01.fasta.gz \\
\hline Ompole & Omphalotus olearius & http://www.ncbi.nlm.nih.gov/Traces/wgs/?download=AHIW01.fasta.gz \\
\hline Oncvol & Onchocerca volvulus & http://www.ncbi.nlm.nih.gov/Traces/wgs/?download=ADBW01.fasta.gz \\
\hline Ophcla & Ophiognomonia clavigignenti & http://www.ncbi.nlm.nih.gov/Traces/wgs/?download=AEGN01.fasta.gz \\
\hline Orenil & Oreochromis niloticus & http://www.ncbi.nlm.nih.gov/Traces/wgs/?download=AERX01.fasta.gz \\
\hline Ornana & Ornithorhynchus anatinus & http://www.ncbi.nlm.nih.gov/Traces/wgs/?download=AAPN01.fasta.gz \\
\hline Oryafe & Orycteropus afer & http://www.ncbi.nlm.nih.gov/Traces/wgs/?download=ALYB01.fasta.gz \\
\hline Orycun & Oryctolagus cuniculus & http://www.ncbi.nlm.nih.gov/Traces/wgs/?download=AAGW02.fasta.gz \\
\hline Orysat & Oryza sativa & http://www.ncbi.nlm.nih.gov/Traces/wgs/?download=AACV01.fasta.gz \\
\hline Orylat & Oryzias latipes & http://www.ncbi.nlm.nih.gov/Traces/wgs/?download=BAAF04.fasta.gz \\
\hline Ostluc & Ostreococcus lucimarinus & ftp://ttp.jgi-psf.org/pub/JGI_data/Ostreococcus_lucimarinus/Olucimarinus.fasta.gz \\
\hline Otogar & Otolemur garnettii & http://www.ncbi.nlm.nih.gov/Traces/wgs/?download=AAQR03.fasta.gz \\
\hline Oviari & Ovis aries & http://www.ncbi.nlm.nih.gov/Traces/wgs/?download=AMGL01.fasta.gz \\
\hline Oxytri & Oxytricha trifallax & http://www.ncbi.nlm.nih.gov/Traces/wgs/?download=AMCR01.fasta.gz \\
\hline Pactan & Pachysolen tannophilus & http://www.ncbi.nlm.nih.gov/Traces/wgs/?download=CAHV01.fasta.gz \\
\hline Pantro & Pan troglodytes & http://www.ncbi.nlm.nih.gov/Traces/wgs/?download=AACZ03.fsa.1.gz \\
\hline Papanu & Papio anubis & http://www.ncbi.nlm.nih.gov/Traces/wgs/?download=AHZZ01.fsa.1.gz \\
\hline Parbra & Paracoccidioides brasiliensis & http://www.ncbi.nlm.nih.gov/Traces/wgs/?download=ABKI01.fasta.gz \\
\hline
\end{tabular}




\begin{tabular}{|c|c|}
\hline Partet & Paramecium tetraurelia \\
\hline Patmin & Patiria miniata \\
\hline Paxrub & Paxillus rubicundulus \\
\hline Pedhum & Pediculus humanus \\
\hline Pelsin & Pelodiscus sinensis \\
\hline Pendig & Penicillium digitatum \\
\hline Peripo & Periglandula ipomoeae \\
\hline Permar & Perkinsus marinus \\
\hline Petmar & Petromyzon marinus \\
\hline Phatri & Phaeodactylum tricornutum \\
\hline Phanod & Phaeosphaeria nodorum \\
\hline Phacar & Phanerochaete carnosa \\
\hline Phlbre & Phlebia brevispora \\
\hline Phlgig & Phlebiopsis gigantea \\
\hline Phlpap & Phlebotomus papatasi \\
\hline Phodac & Phoenix dactylifera \\
\hline Phybla & Phycomyces blakesleeanus \\
\hline Phypat & Physcomitrella patens \\
\hline Phyinf & Phytophthora infestans \\
\hline Pickud & Pichia kudriavzevii \\
\hline Pilcro & Piloderma croceum \\
\hline Pinfuc & Pinctada fucata \\
\hline Pirind & Piriformospora indica \\
\hline Pir_sp & Piromyces sp \\
\hline Pismic & Pisolithus microcarpus \\
\hline Plaviv & Plasmodium vivax \\
\hline Pleost & Pleurotus ostreatus \\
\hline Plicri & Plicaturopsis crispa \\
\hline Pogbar & Pogonomyrmex barbatus \\
\hline Polpal & Polysphondylium pallidum \\
\hline Ponabe & Pongo abelii \\
\hline Poptri & Populus trichocarpa \\
\hline Pospla & Postia placenta \\
\hline Pripac & Pristionchus pacificus \\
\hline Procap & Procavia capensis \\
\hline Pruper & Prunus persica \\
\hline Psemul & Pseudo-nitzschia multiseries \\
\hline Psecub & Pseudoperonospora cubensis \\
\hline Ptevam & Pteropus vampyrus \\
\hline Pucgra & Puccinia graminis \\
\hline Punstr & Punctularia strigosozonata \\
\hline Punnye & Pundamilia nyererei \\
\hline Pyrter & Pyrenophora teres \\
\hline
\end{tabular}

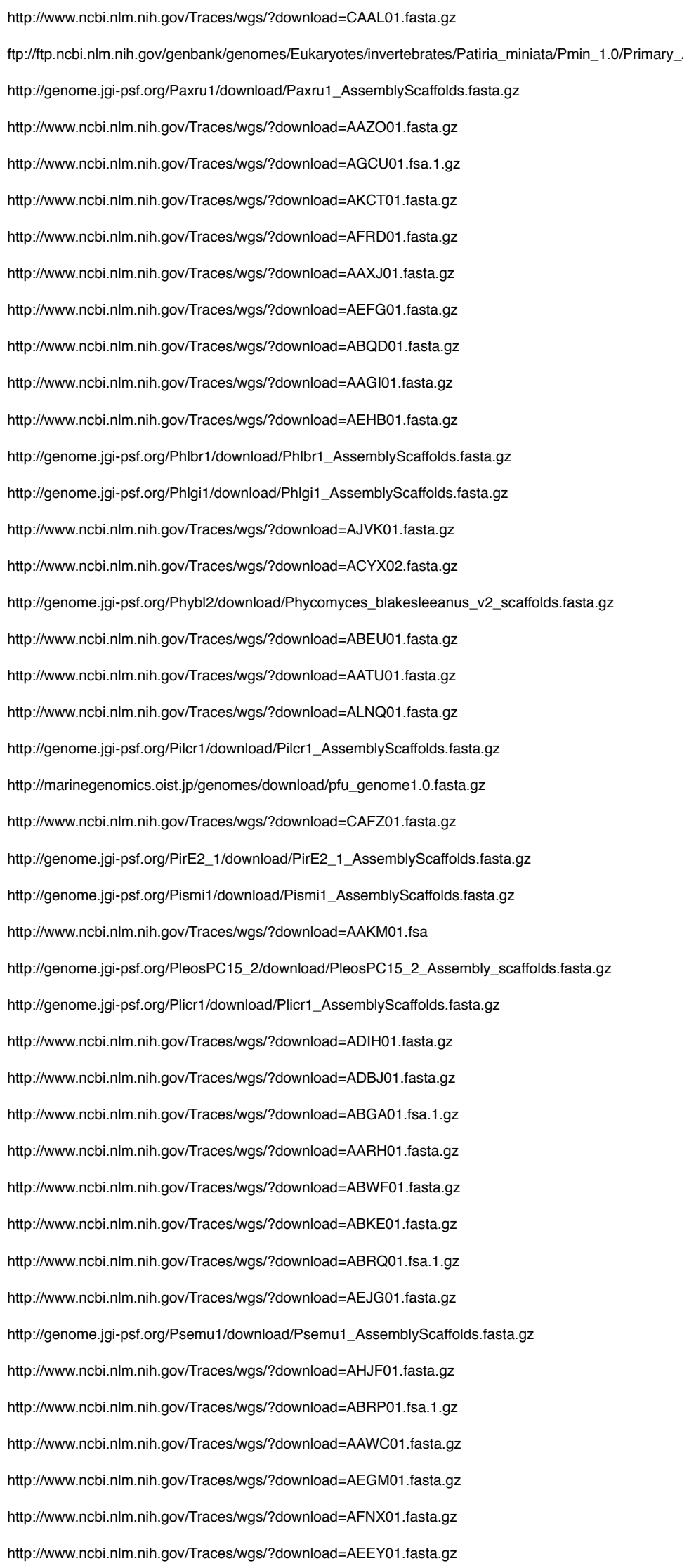




\begin{tabular}{|c|c|c|}
\hline Pytult & Pythium ultimum & http://www.ncbi.nlm.nih.gov/Traces/wgs/?download=ADOS01.fasta.gz \\
\hline Pytmol & Python molurus & http://www.ncbi.nlm.nih.gov/Traces/wgs/?download=AEQU01.fsa.1.gz \\
\hline Ratnor & Rattus norvegicus & http://www.ncbi.nlm.nih.gov//races/wgs/?download=AABR06.fsa.1.gz \\
\hline Rhaeso & Rhamphochromis esox & http://www.ncbi.nlm.nih.gov/Traces/wgs/?download=ABPN01.fasta.gz \\
\hline Rhopro & Rhodnius prolixus & http://www.ncbi.nlm.nih.gov//races/wgs/?download=ACPB02.fasta.gz \\
\hline Rhotor & Rhodosporidium toruloides & http://www.ncbi.nlm.nih.gov/Traces/wgs/?download=AJMJ01.fasta.gz \\
\hline Rhoglu & Rhodotorula glutinis & http://www.ncbi.nlm.nih.gov/Traces/wgs/?download=AEVR01.fasta.gz \\
\hline Riccom & Ricinus communis & http://www.ncbi.nlm.nih.gov/Traces/wgs/?download=AASG02.fasta.gz \\
\hline Saccer & Saccharomyces cerevisiae & http://www.ncbi.nlm.nih.gov/Traces/wgs/?download=ACVY01.fasta.gz \\
\hline Sackow & Saccoglossus kowalevskii & http://www.ncbi.nlm.nih.gov/Traces/wgs/?download=ACQM01.fasta.gz \\
\hline Saibol & Saimiri boliviensis & http://www.ncbi.nlm.nih.gov/Traces/wgs/?download=AGCE01.fsa.1.gz \\
\hline Saicom & Saitoella complicata & http://www.ncbi.nlm.nih.gov/Traces/wgs/?download=BACD01.fasta.gz \\
\hline Salsal & Salmo salar & http://www.ncbi.nlm.nih.gov/Traces/wgs/?download=AGKD01.fasta.gz \\
\hline Sal_sp & Salpingoeca sp & http://www.ncbi.nlm.nih.gov/Traces/wgs/?download=ACSY01.fasta.gz \\
\hline Sappar & Saprolegnia parasitica & http://www.ncbi.nlm.nih.gov/Traces/wgs/?download=ADCG01.fasta.gz \\
\hline Sarhar & Sarcophilus harrisii & http://www.ncbi.nlm.nih.gov/Traces/wgs/?download=AEFK01.fsa.1.gz \\
\hline Schman & Schistosoma mansoni & $\mathrm{ftp}: / /$ ttp.sanger.ac.uk/pub/pathogens/Schistosoma/mansoni/genome/Assembly-v5/sma_v5.0.chr.fa.gz \\
\hline Schcom & Schizophyllum commune & http://www.ncbi.nlm.nih.gov/Traces/wgs/?download=ADMJ01.fasta.gz \\
\hline Schjap & Schizosaccharomyces japonicus & http://www.ncbi.nlm.nih.gov/Traces/wgs/?download=AATM01.fasta.gz \\
\hline Schmed & Schmidtea mediterranea & http://www.ncbi.nlm.nih.gov/Traces/wgs/?download=AAWT01.fasta.gz \\
\hline Sclcit & Scleroderma citrinum & http://genome.jgi-psf.org/Sclci1/download/Sclci1_AssemblyScaffolds.fasta.gz \\
\hline Sclscl & Sclerotinia sclerotiorum & http://www.ncbi.nlm.nih.gov/Traces/wgs/?download=AAGT01.fasta.gz \\
\hline Sebver & Sebacina vermifera & http://genome.jgi-psf.org/Sebve1/download/Sebve1_AssemblyScaffolds.fasta.gz \\
\hline Selmoe & Selaginella moellendorffii & http://www.ncbi.nlm.nih.gov/Traces/wgs/?download=ADFJ01.fasta.gz \\
\hline Serlac & Serpula lacrymans & http://www.ncbi.nlm.nih.gov/Traces/wgs/?download=AEQB01.fasta.gz \\
\hline Setita & Setaria italica & http://www.ncbi.nlm.nih.gov/Traces/wgs/?download=AGNK01.fasta.gz \\
\hline Settur & Setosphaeria turcica & http://genome.jgi-psf.org/Settu1/download/Settu1_AssemblyScaffolds.fasta.gz \\
\hline Sollyc & Solanum lycopersicum & http://www.ncbi.nlm.nih.gov/Traces/wgs/?download=AEKE02.fasta.gz \\
\hline Solinv & Solenopsis invicta & http://www.ncbi.nlm.nih.gov/Traces/wgs/?download=AEAQ01.fasta.gz \\
\hline Sormac & Sordaria macrospora & http://www.ncbi.nlm.nih.gov/Traces/wgs/?download=CABT02.fasta.gz \\
\hline Sorara & Sorex araneus & http://www.ncbi.nlm.nih.gov/Traces/wgs/?download=AALT02.fsa.1.gz \\
\hline Sorbic & Sorghum bicolor & http://www.ncbi.nlm.nih.gov/Traces/wgs/?download=ABXC01.fasta.gz \\
\hline Spapas & Spathaspora passalidarum & http://www.ncbi.nlm.nih.gov/Traces/wgs/?download=AEIK01.fasta.gz \\
\hline Spetri & Spermophilus tridecemlineatus & http://www.ncbi.nlm.nih.gov/Traces/wgs/?download=AGTP01.fsa.1.gz \\
\hline Sphste & Sphaerobolus stellatus & http://genome.jgi-psf.org/Sphst1/download/Sphst1_AssemblyScaffolds.fasta.gz \\
\hline Spipun & Spizellomyces punctatus & http://www.ncbi.nlm.nih.gov/Traces/wgs/?download=ACOE01.fasta.gz \\
\hline Sporos & Sporobolomyces roseus & ftp://ftp.jgi-psf.org/pub/JGI_data/Sporobolomyces_roseus/assembly/v1.0/Sporobolomyces_roseus.allmas \\
\hline Stehir & Stereum hirsutum & http://www.ncbi.nlm.nih.gov/Traces/wgs/?download=AEGX01.fasta.gz \\
\hline Strmar & Strigamia maritima & http://www.ncbi.nlm.nih.gov/Traces/wgs/?download=AFFK01.fasta.gz \\
\hline Strpur & Strongylocentrotus purpuratus & ftp://ftp.ncbi.nlm.nih.gov/genbank/genomes/Eukaryotes/invertebrates/Strongylocentrotus_purpuratus/Spl \\
\hline Strrat & Strongyloides ratti & http://www.ncbi.nlm.nih.gov/Traces/wgs/?download=CACX01.fasta.gz \\
\hline Stylem & Stylonychia lemnae & http://www.ncbi.nlm.nih.gov/Traces/wgs/?download=ADNZ01.fasta.gz \\
\hline Suilut & Suillus luteus & http://genome.jgi-psf.org/Suilu1/download/Suilu1_AssemblyScaffolds.fasta.gz \\
\hline
\end{tabular}




\begin{tabular}{|c|c|c|}
\hline Susscr & Sus scrofa & http://www.ncbi.nlm.nih.gov/Traces/wgs/?download=AEMK01.fsa.1.gz \\
\hline Taegut & Taeniopygia guttata & http://www.ncbi.nlm.nih.gov/Traces/wgs/?download=ABQF01.fsa.1.gz \\
\hline Takrub & Takifugu rubripes & http://www.ncbi.nlm.nih.gov/Traces/wgs/?download=CAAB02.fasta.gz \\
\hline Talmar & Talaromyces marneffei & http://www.ncbi.nlm.nih.gov/Traces/wgs/?download=ABAR01.fasta.gz \\
\hline Tapdef & Taphrina deformans & http://www.ncbi.nlm.nih.gov/Traces/wgs/?download=CAHR02.fasta.gz \\
\hline Tarsyr & Tarsius syrichta & http://www.ncbi.nlm.nih.gov/Traces/wgs/?download=ABRT01.fsa.1.gz \\
\hline Tetthe & Tetrahymena thermophila & http://www.ncbi.nlm.nih.gov/Traces/wgs/?download=AAGF03.fasta.gz \\
\hline Teturt & Tetranychus urticae & http://www.ncbi.nlm.nih.gov/Traces/wgs/?download=CAEY01.fasta.gz \\
\hline Tetnig & Tetraodon nigroviridis & http://www.ncbi.nlm.nih.gov/Traces/wgs/?download=CAAE01.fasta.gz \\
\hline Thapse & Thalassiosira pseudonana & http://www.ncbi.nlm.nih.gov/Traces/wgs/?download=AAFD02.fasta.gz \\
\hline Thepar & Theileria parva & http://www.ncbi.nlm.nih.gov/Traces/wgs/?download=AAGK01.fasta.gz \\
\hline Thecac & Theobroma cacao & http://www.ncbi.nlm.nih.gov/Traces/wgs/?download=CACC01.fasta.gz \\
\hline Thelan & Thermomyces lanuginosus & http://www.ncbi.nlm.nih.gov/Traces/wgs/?download=ANHP01.fasta.gz \\
\hline Thiter & Thielavia terrestris & http://genome.jgi-psf.org/Thite2/download/Thite2_AssemblyScaffolds.fasta.gz \\
\hline Toxgon & Toxoplasma gondii & http://www.ncbi.nlm.nih.gov/Traces/wgs/?download=ABPA01.fasta.gz \\
\hline Traver & Trametes versicolor & http://www.ncbi.nlm.nih.gov/Traces/wgs/?download=AEJI01.fasta.gz \\
\hline Tremes & Tremella mesenterica & http://www.ncbi.nlm.nih.gov/Traces/wgs/?download=AFVY01.fasta.gz \\
\hline Tricas & Tribolium castaneum & http://www.ncbi.nlm.nih.gov/Traces/wgs/?download=AAJJ01.fasta.gz \\
\hline Triman & Trichechus manatus & http://www.ncbi.nlm.nih.gov/Traces/wgs/?download=AHIN01.fsa.1.gz \\
\hline Trispi & Trichinella spiralis & http://www.ncbi.nlm.nih.gov/Traces/wgs/?download=ABIR02.fasta.gz \\
\hline Triree & Trichoderma reesei & http://www.ncbi.nlm.nih.gov/Traces/wgs/?download=AAIL02.fasta.gz \\
\hline Trivag & Trichomonas vaginalis & http://www.ncbi.nlm.nih.gov/Traces/wgs/?download=AAHC01.fasta.gz \\
\hline Trirub & Trichophyton rubrum & http://www.ncbi.nlm.nih.gov/Traces/wgs/?download=ACPH01.fasta.gz \\
\hline Triadh & Trichoplax adhaerens & http://www.ncbi.nlm.nih.gov/Traces/wgs/?download=ABGP01.fasta.gz \\
\hline Triasa & Trichosporon asahii & http://www.ncbi.nlm.nih.gov/Traces/wgs/?download=AMBO01.fasta.gz \\
\hline Triaes & Triticum aestivum & http://www.ncbi.nlm.nih.gov/Traces/wgs/?download=AEOM01.fasta.gz \\
\hline Trycru & Trypanosoma cruzi & http://www.ncbi.nlm.nih.gov/Traces/wgs/?download=AAHK01.fasta.gz \\
\hline Tubmel & Tuber melanosporum & http://www.ncbi.nlm.nih.gov/Traces/wgs/?download=CABJ01.fasta.gz \\
\hline Tulcal & Tulasnella calospora & http://genome.jgi-psf.org/Tulca1/download/Tulca1_AssemblyScaffolds.fasta.gz \\
\hline Tupbel & Tupaia belangeri & http://www.ncbi.nlm.nih.gov/Traces/wgs/?download=AAPY01.fsa.1.gz \\
\hline Turtru & Tursiops truncatus & http://www.ncbi.nlm.nih.gov/Traces/wgs/?download=ABRN02.fsa.1.gz \\
\hline Uncree & Uncinocarpus reesii & http://www.ncbi.nlm.nih.gov/Traces/wgs/?download=AAIW01.fasta.gz \\
\hline Usthor & Ustilago hordei & http://www.ncbi.nlm.nih.gov/Traces/wgs/?download=CAGI01.fasta.gz \\
\hline Vanpol & Vanderwaltozyma polyspora & http://www.ncbi.nlm.nih.gov/Traces/wgs/?download=AAZN01.fasta.gz \\
\hline Vardes & Varroa destructor & http://www.ncbi.nlm.nih.gov/Traces/wgs/?download=ADDG01.fasta.gz \\
\hline Vavcul & Vavraia culicis & http://www.ncbi.nlm.nih.gov/Traces/wgs/?download=AEUG01.fasta.gz \\
\hline Verdah & Verticillium dahliae & http://www.ncbi.nlm.nih.gov/Traces/wgs/?download=ABJE01.fasta.gz \\
\hline Vicpac & Vicugna pacos & http://www.ncbi.nlm.nih.gov/Traces/wgs/?download=ABRR01.fsa.1.gz \\
\hline Vitvin & Vitis vinifera & http://www.ncbi.nlm.nih.gov/Traces/wgs/?download=CAAP03.fasta.gz \\
\hline Vitcor & Vittaforma corneae & http://www.ncbi.nlm.nih.gov/Traces/wgs/?download=AEYK01.fasta.gz \\
\hline Volcar & Volvox carteri & http://www.ncbi.nlm.nih.gov/Traces/wgs/?download=ACJH01.fasta.gz \\
\hline Walseb & Wallemia sebi & http://www.ncbi.nlm.nih.gov/Traces/wgs/?download=AFQX01.fasta.gz \\
\hline Wicano & Wickerhamomyces anomalus & http://www.ncbi.nlm.nih.gov/Traces/wgs/?download=AEGI02.fasta.gz \\
\hline
\end{tabular}




$\begin{array}{lll}\text { Wolcoc } & \text { Wolfiporia cocos } & \text { http://genome.jgi-psf.org/Wolco1/download/Wolco1_AssemblyScaffolds.fasta.gz } \\ \text { Wucban } & \text { Wuchereria bancrofti } & \text { http://www.ncbi.nlm.nih.gov/Traces/wgs/?download=ADBV01.fasta.gz } \\ \text { Xanpar } & \text { Xanthoria parietina } & \text { http://genome.jgi-psf.org/Xanpa1/download/Xanpa1_AssemblyScaffolds.fasta.gz } \\ \text { Xentro } & \text { Xenopus tropicalis } & \text { http://www.ncbi.nlm.nih.gov/Traces/wgs/?download=AAMC02.fsa.1.gz } \\ \text { Xipmac } & \text { Xiphophorus maculatus } & \text { http://www.ncbi.nlm.nih.gov/Traces/wgs/?download=AGAJ01.fasta.gz } \\ \text { Zascel } & \text { Zasmidium cellare } & \text { http://genome.jgi-psf.org/Zasce1/download/Zasce1_AssemblyScaffolds.fasta.gz } \\ \text { Zeamay } & \text { Zea mays } & \text { http://www.ncbi.nlm.nih.gov/Traces/wgs/?download=AECO01.fasta.gz } \\ \text { Zymard } & \text { Zymoseptoria ardabiliae } & \text { http://www.ncbi.nlm.nih.gov/Traces/wgs/?download=AFIV01.fasta.gz }\end{array}$




\title{
CHAPTER 3
}

\section{Evolutionary and biogeographical patterns of barnacles from deep-sea hydrothermal vents}

\author{
Santiago Herrera ${ }^{1,2}$, Hiromi Watanabe ${ }^{3}$, Timothy M. Shank ${ }^{2}$ \\ ${ }^{1}$ Massachusetts Institute of Technology, 77 Massachusetts Avenue, Cambridge, MA 02139, USA \\ ${ }^{2}$ Biology Department, Woods Hole Oceanographic Institution, 266 Woods Hole Road, Woods Hole, MA \\ 02543, USA \\ ${ }^{3}$ Institute of Biogeosciences, Japan Agency for Marine-Earth Science and Technology, Yokosuka, \\ Kanagawa, Japan
}

\begin{abstract}
The characterization of evolutionary and biogeographical patterns is of fundamental importance to identify factors driving biodiversity. Due to their widespread but discontinuous distribution, deep-sea hydrothermal vent barnacles represent an excellent model for testing biogeographic hypotheses regarding the origin, dispersal, and diversity of modern vent fauna. Here we characterize the global genetic diversity of vent barnacles to infer their time of radiation, place of origin, mode of dispersal, and diversification. Our approach was to target a suite of multiple loci in samples representing seven out of the eight described genera. We also performed restriction-site associated DNA sequencing on individuals from each species. Phylogenetic inferences and topology hypothesis tests indicate that vent barnacles have colonized deep-sea hydrothermal vents at least twice in history. Consistent with preliminary estimates, we find a likely radiation of barnacles in vent ecosystems during the Cenozoic. Our analyses suggest that the western Pacific was the place of origin of the major vent barnacle lineage, followed by circumglobal colonization eastward through the southern hemisphere during the Neogene. The inferred time of radiation rejects classic hypotheses of antiquity of vent taxa. The timing and the mode of origin, radiation and dispersal are consistent with recent inferences made for other deep-sea taxa, including non-vent species, and are correlated with the occurrence of major geological events and mass extinctions. Thus, we suggest that the geological processes and dispersal mechanisms discussed here can explain current distribution patterns of many other marine taxa and have played an important role shaping deep-sea
\end{abstract}


faunal diversity. These results also constitute critical baseline data with which to assess potential effects of anthropogenic disturbances on deep-sea ecosystems.

\section{INTRODUCTION}

The characterization of evolutionary and biogeographical patterns is of fundamental importance for identifying the factors that shape the ranges of deep-sea taxa, and that ultimately drive biodiversity patterns in the ocean (McClain \& Mincks 2010). This is particularly relevant in the light of the increasing interest in commercial resource extraction in the deep-sea (Thurber et al. 2014). Mining of seafloor massive sulphide deposits at deep-sea hydrothermal vent fields has become one of the main industrial targets for exploitation (Boschen et al. 2013). Understanding the biodiversity contained in these areas and its connection with the fauna found elsewhere is critical for assessing the potential impacts of exploiting these mineral resources (Van Dover 2010; Van Dover et al. 2012). Although organisms living at deep-sea hydrothermal vents have adapted to cope with natural disturbances inherent to these ephemeral habitats, the intensity and frequency at which these occur can vary greatly depending on the particular geophysical nature of each system (Baker \& German 2004). Thus, disturbance from mining could have additive or synergistic effects to natural disturbances at unprecedented scales, which could potentially lead to significant losses of biodiversity (Van Dover 2010). Due to their widespread distribution (Fig. 1), vent barnacles represent an excellent model for testing hypotheses regarding the historical biogeographic patterns of origin, dispersal, and current diversity of modern deep-sea chemosynthetic fauna; therefore, barnacles hold the promise of providing critical baseline data with which to assess potential effects of anthropogenic disturbances on deep-sea ecosystems.

Barnacles (Cirripedia Burmeister, 1834) are some of the most conspicuous organisms in deep-sea hydrothermal vent ecosystems worldwide. These sessile crustaceans can be found in active vent fields in most of the major spreading ridge systems and island arcs worldwide (Fig. 1), including the Central Indian Ridge (Van Dover et al. 2001; Nakamura et al. 2012), Southwest Indian Ridge (Tao et al. 2011), East Scotia Ridge (Rogers et al. 2012), northern and southern East Pacific Rise (Newman 1979; Jones 1993), Pacific-Antarctic Ridge (Southward 2005), Izu-Ogasawara Arc (Ohno et al. 1996), Okinawa Trough (Ohta 1990), Mariana Trough (Hessler \& Lonsdale 1991), Sangihe Talaud (Herrera et al. 2010; Shank et al. 2010), Manus Basin (Tufar 1990), Edison Seamount (Tunnicliffe \& Southward 2004), North

Fiji Basin (Desbruyeres et al. 1994), Lau Basin (Southward \& Newman 1998), Kermadec Arc (Buckeridge 2000), and are likely to be present in other unexplored areas. Hydrothermal vent barnacles inhabit areas of low-temperature diffuse fluid flow. Populations can reach high densities with high 
biomass at over 1500 individuals per square meters (Tunnicliffe \& Southward 2004; Marsh et al. 2012), playing key roles in vent communities as micro-habitat engineers and funnelling the flow of energy through ecosystems from primary producers to higher trophic levels (Southward \& Newman 1998; Van Dover 2002; Tunnicliffe \& Southward 2004; Cubelio et al. 2007; Rogers et al. 2012; Reid et al. 2013).

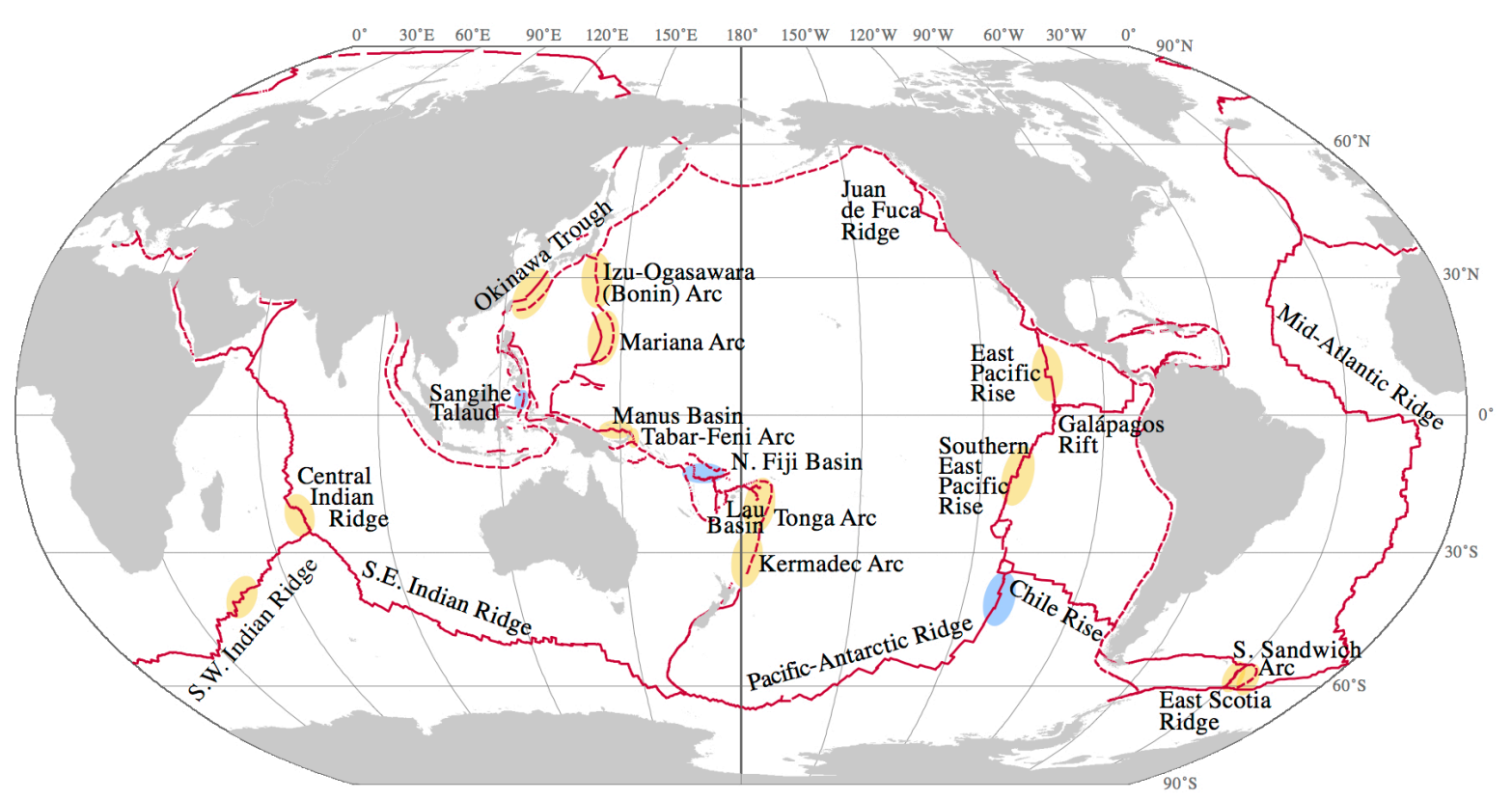

Figure 1. Global distribution map of hydrothermal vent barnacles. Ovals indicate regions where hydrothermal vent barnacles have been described (yellow: regions sampled in this study; blue: regions not sampled in this study). Red lines indicate active tectonic margins (solid lines: spreading centers; dotted lines: subduction zones).

Hydrothermal vent barnacles are presently grouped into four families belonging to the orders Pedunculata Lamarck, 1818 (suborder Scalpellomorpha, family Eolepadidae; commonly known as stalked or gooseneck barnacles) and Sessilia Lamarck, 1818 (suborder Verucomorpha, family Neoverrucidae; suborder Brachylepadomorpha, family Neobrachylepadidae; and suborder Balanomorpha, family Chionelasmatidae; commonly known as acorn barnacles) (Newman et al. 2006). There are approximately 13 described vent barnacle species, with several new species awaiting description (Newman et al. 2006). A molecular phylogenetic study of the Cirripedia, employing nuclear ribosomal genes and the histone $H 3$ gene, indicates that these morphologically-based taxonomic groupings (orders) are polyphyletic and thus incongruent with evolutionary history (Pérez-Losada et al. 2008). These results, together with those from (Linse et al. 2013), also suggest that vent barnacles form a monophyletic clade that likely originated in the Cretaceous; however, the possibility of a single origin remains an open question due to the paucity of 
taxonomic sampling in that study. Furthermore, the relationships among morphospecies of vent barnacles also remain unresolved due to the low variability of markers examined to date.

Many putative species of vent barnacles appear to be restricted to particular ridge systems and neighboring arc and back-arc basins, and significant population structure has also been found at these scales (Watanabe et al. 2005). Together these observations suggest a role of habitat discontinuity as an important mechanism of speciation. By far, the region of highest diversity of putative chemosynthetic barnacle species (measured as species richness) is the western Pacific, which is considered the center of their distribution and possible place of origin (Newman et al. 2006). The western Pacific is also considered a biodiversity hotspot and potential place of origin of many modern groups of terrestrial and marine organisms, including deep-sea taxa (Cairns 2007; Carpenter et al. 2011; Herrera et al. 2012). In a similar way, a recent biogeographic analyses using network theory hypothesizes a possible ancestral position of the western Pacific for modern fauna associated with hydrothermal vents, having exclusive edge connections (indicating faunal similarity possible exchange paths) with the Northeast Pacific, the East Pacific Rise and the Indian Ocean (Moalic et al. 2011).

In this study, we aim to characterize the global genetic diversity, evolutionary and biogeographic history of barnacles from deep-sea hydrothermal vents. Our approach was to build on previous phylogenetic studies by significantly expanding the taxonomic sampling and number of genetic markers. We targeted one mitochondrial gene region, the cytochrome c oxidase subunit I ( $\operatorname{cox} I)$, and two nuclear gene regions, the large ribosomal sub-unit $28 \mathrm{~S}$, and the histone $H 3$ gene, obtaining complete sequences for 94 individuals, representing seven out of the eight described genera, from 18 vent fields worldwide. We also performed restriction-site associated DNA sequencing (RAD-seq) on individuals from each identified species. Here we: (1) test the hypothesis of monophyly (i.e., a single evolutionary origin) of barnacles from deep-sea hydrothermal vents; (2) infer the place and time of origin and radiation of vent barnacles in geologic time; (3) infer historical patterns of dispersal and colonization of vent barnacle taxa worldwide; and (4) identify species boundaries and compare them to current morphospecies hypotheses.

\section{METHODS}

Morphological identifications were performed on 94 barnacle specimens (Table S1) from deep-sea hydrothermal vents using stereo-microscopy and species descriptions as references. Individuals were collected from the Central Indian Ridge, East Pacific Rise, southern East Pacific Rise, Southwest Indian 
Ridge, East Scotia Ridge, Mariana Trough, the Kermadec Arc, Lau Basin, Tonga Arc, Manus Basin, IzuOgasawara (Bonin) Arc, and the Okinawa Trough.

Partial DNA sequences of one mitochondrial (cytochrome c oxidase subunit I) and two nuclear markers (histone $H 3$ gene and the ribosomal large sub-unit 28S) were generated for each individual. Additional sequences from the Superorder Thoracica Darwin, 1854 were retrieved from GenBank (http://www.ncbi.nlm.nih.gov/genbank/) and included in the analyses (Table S2).

Restriction-site Associated DNA sequencing (RAD-seq) (Baird et al. 2008) was performed on selected individuals from each morphospecies (Table S1) to obtain a genome-wide set of markers that could be used to infer a robust backbone of the vent barnacle phylogenetic tree, and to compare to topologies obtained from species-tree analyses of traditional Sanger-based markers.

\section{Molecular laboratory methods}

Total genomic DNA was extracted from tissue samples by: (1) digesting the tissue in $2 \%$ CTAB buffer (Teknova) with proteinase K and RNAse A/T1 (Fermentas) for 1 hour, (2) separating nucleic acids with chloroform: isoamyl alcohol (24:1) (Fermentas) and phenol: chloroform: isoamyl alcohol (25:24:1, Tris buffered at $\mathrm{pH}$ 8.0) (Fermentas), (3) precipitating nucleic acids with 100\% ethanol (1:1), and (4) washing the precipitate twice with $70 \%$ ethanol. Polymerase chain reactions of traditional Sanger-based markers were prepared to a final volume of $25 \mu 1$ ( $1 \mu \mathrm{l}$ of template) resulting in the following final concentrations of reagents and enzymes: $1 \mathrm{X}$ GoTaq Flexi Buffer (Promega), $2.5 \mathrm{X}$ BSA, $1.0 \mathrm{mM}$ dNTPs (0.25 mM each), $2.0 \mathrm{mM} \mathrm{MgCl} 2,1 \mathrm{U}$ Taq polymerase (GoTaq, Promega), and $0.3 \mu \mathrm{M}$ of each primer. Primer pairs used for amplifications were: 28SF_330 5'-CGTGAAGCTGCCAVTATGG-3' (designed in this study) \& 28S_B (Whiting 2002) for 28S, H3F \& H3R (Colgan et al. 1998) for H3, and LCO1490 \& HC02198 (Folmer et al. 1994) for coxI. Negative controls were included in every experiment to test for contamination. The reactions were carried out with an initial denaturation step of $5 \mathrm{~min}$ at $94{ }^{\circ} \mathrm{C}, 32$ cycles $\left(35\right.$ for coxl) of $60 \mathrm{~s}$ at $94{ }^{\circ} \mathrm{C}, 90 \mathrm{~s}$ at $48{ }^{\circ} \mathrm{C}$, and $90 \mathrm{~s}$ at $72{ }^{\circ} \mathrm{C}$, and a final elongation step of 10 min at $72{ }^{\circ} \mathrm{C}$. PCR products were cleaned using the MinElute PCR Purification Kit (Qiagen) following manufacturer's protocols. Cycle-sequencing reactions were performed using the ABI BigDye Terminator v3.1 kit (Life Technologies Corp.), following manufacturer protocols. Subsequent purification was done through isopropanol precipitation. Automated sequencing was completed using a 3730xl DNA analyzer (Life technologies Corp.) at the Josephine Bay Paul Center of the Marine Biological Laboratory. Complementary chromatograms were assembled and edited using Geneious v6.1.6 (Drummond et al. 2011). 
Concentration-normalized genomic DNA was submitted to Floragenex Inc. (Eugene, OR) for library preparation and RAD sequencing. Individual libraries were produced from DNA digested with a highfidelity SbfI restriction enzyme, which is predicted to cut approximately between 5,000 and 15,000 times in the genome of a thoracican barnacle (Table S3). This predicted range was obtained using the observed frequency of the SbfI recognition sequence, and its probability using a trinucleotide composition model, in the genome of the crustacean Daphnia pulex (Herrera et al. Chapter 2). Ranges of genome size for barnacles were obtained from the Animal Genome Size Database (http://ww.genomesize.com). Barcode tags were 10-base pairs long. Libraries were sequenced by 96 -multiplex on a single lane of an Illumina Hi-Seq 2000 sequencer.

\section{Alignments, saturation analysis and model selection}

Each set of sequences for Sanger-based markers was aligned independently using MAFFT (Katoh et al. 2002), employing the G-INS-i and Q-INS-i algorithms (gap opening penalty $=1.53$, offset value $=0.07$ ) for protein coding and ribosomal regions, respectively. To correct possible mistakes, all alignments of protein coding sequences were visually inspected and translated to amino acids in Geneious v6.1.6 (Drummond et al. 2011). No unusual stop codons, misplaced reading frames or suspicious substitutions were identified, indicating amplification of nuclear pseudogenes was unlikely (Lopez et al. 1994; Bensasson et al. 2001). Possible substitution saturation in the DNA sequences was evaluated by implementing the Xia test (Xia et al. 2003), as implemented in DAMBE v5.3.48 (Xia 2013), and by plotting genetic distances (K80 model) against the number of transitions and transversions. Saturation in codon partitions was also evaluated for each coding region.

\section{Phylogenetic Inferences}

Non-saturated datasets from individual Sanger-based markers were analyzed in RAxML-HPC2 v8.0 (Stamatakis 2006), as implemented in the CIPRES Science Gateway v3.3 (http://www.phylo.org), for a first-pass phylogenetic inference using the maximum likelihood optimality criterion. Branch support was assessed by 500 bootstrap replicates. A Thoracica-wide concatenated dataset was also analysed in this program. Only outgroups with data for at least two of the three markers were included in the concatenated dataset. Phylogenetic estimation through Bayesian inference was performed with these datasets in MrBayes v3.2.2 (Ronquist et al. 2012), as implemented in the CIPRES Science Gateway v3.3. Models of nucleotide substitution were selected for each non-saturated gene region using JModeltest v2.0 (Darriba et al. 2012), following the Bayesian Information Criterion (Table S4). Four independent analyses of 200 million Markov Chain Monte Carlo (MCMC) generations (4 chains) were run with a sampling frequency 
of 20 thousand generations (burn-in $=25 \%$ ). Combined analyses were performed with explicit character partitions for each concatenated region, along with their independently selected models of evolution. State frequencies were allowed to vary under a flat Dirichlet prior distribution to account for the rate variation among partitions. Nucleotide frequencies, substitution rates, gamma shape and invariant site proportion parameters were unlinked across partitions. Default prior distribution settings were assumed for all other parameters. MCMC runs were analyzed with the programs Tracer v1.5 (Rambaut \& Drummond 2007) and AWTY (http://ceb.csit.fsu.edu/awty) (Nylander et al. 2008). Convergence among independent runs was supported by observed values of standard deviation of partition frequencies $(<0.01)$, potential scale reduction factors (PSRF) (ca. 1.00), and effective sample sizes (EES) (>200), in addition to high correlations between runs and the flat shapes of the stationary posterior distribution traces of each parameter.

\section{Topological Hypothesis Testing}

To test the hypothesis that barnacles from deep-sea hydrothermal vents form a monophyletic group, we performed a Bayes factor comparison (Kass \& Raftery 1995) between this topological hypothesis and the alternative hypothesis of non-monophyly of the group using the Thoracica-wide concatenated dataset. The marginal likelihood for each topology model was estimated through the stepping-stone method (Fan et al. 2011; Xie et al. 2011) in MrBayes using 50 steps. The estimation was performed in two independent runs of 100 million generations, with a diagnostic frequency of 1 million generations, for each topology model. All other parameters were set to default. Convergence among runs was diagnosed by the standard deviation of partition frequencies $(<0.01)$.

\section{Divergence Time Estimations}

Time-calibration of the phylogenetic hypothesis was carried out through a Bayesian-MCMC joint estimation of phylogeny and divergence times in BEAST v1.7.5 (Drummond et al. 2012), as implemented in the CIPRES Science Gateway v3.3, using the Thoracica-wide concatenated Sanger-based markers dataset. Variation in mutation rates among branches was allowed by assuming an uncorrelated relaxed lognormal molecular clock model. The Yule constant speciation rate model and no extinction (Yule 1925), the Birth-Death constant speciation and extinction rates model (Gernhard 2008), and the BirthDeath constant speciation and extinction rates with incomplete taxonomic sampling model (Stadler 2009) were tested as tree priors. Unlinked character partitions were set for each concatenated region, along with their independently selected models of evolution. Three fossil calibration points (C1, C2, and C7) were selected from the studies by Pérez-Losada et al. (2008) and Linse et al. (2013) based on well-supported topological congruencies with our phylogenetic hypothesis. Fossil ages were used as lower boundary 
constraints assuming prior exponential distributions with mean values of 25 my. Default prior distribution settings were assumed for all other parameters. Three independent MCMC analyses were run for 200 million generations with a sampling frequency of 20 thousand. Convergence diagnostics were examined for the combined runs in Tracer as mentioned above. Most probable trees, after $25 \%$ burn-in, were summarized into a maximum clade credibility tree with median node heights using TreeAnnotator v1.7.1 (Drummond et al. 2012).

\section{Historical Biogeography}

To infer historical patterns of dispersal in deep-sea hydrothermal vent barnacle lineages, we performed a Bayesian reconstruction of discrete character states of geographic location for ancestral nodes (Lemey et al. 2009) using BEAST v1.7.5 (Heled \& Drummond 2010). In this framework, the geographical sampling locations were mapped to the time-scaled phylogenetic tree. Parameters for tree inference were as described above.

\section{Species Delimitation}

To identify species boundaries for vent barnacles in Clade A (see Results section), we employed generalized mixed Yule-coalescent (GMYC) likelihood method (Pons et al. 2006; Monaghan et al. 2009; Fujisawa \& Barraclough 2013), with a single threshold, as implemented in the SPLITS R-package (available from http://r-forge.r-project.org/ projects/splits/). This method estimates species boundaries by identifying increases in branching rates that are characteristic of transition points between interspecific speciation-extinction processes and intraspecific coalescent processes.

\section{Species Tree Estimation}

Bayesian analyses of species-trees estimation for vent barnacle species identified in Clade A (see Results section) were carried out using data from the Sanger-based markers in the program *BEAST v1.7.5 (Heled \& Drummond 2010). This approach was employed to take into account evolutionary coalescent processes and gene tree heterogeneity, and to evaluate the effects of gene-concatenation on the phylogenetic inference (Brito \& Edwards 2008; Edwards 2008). Species were defined after the species delimitation analyses. Unlinked character, clock, and tree partitions were set for each marker, along with their independently selected models of evolution. We assumed a piecewise linear and constant root population size model. Other parameters for tree inference were as described above. 


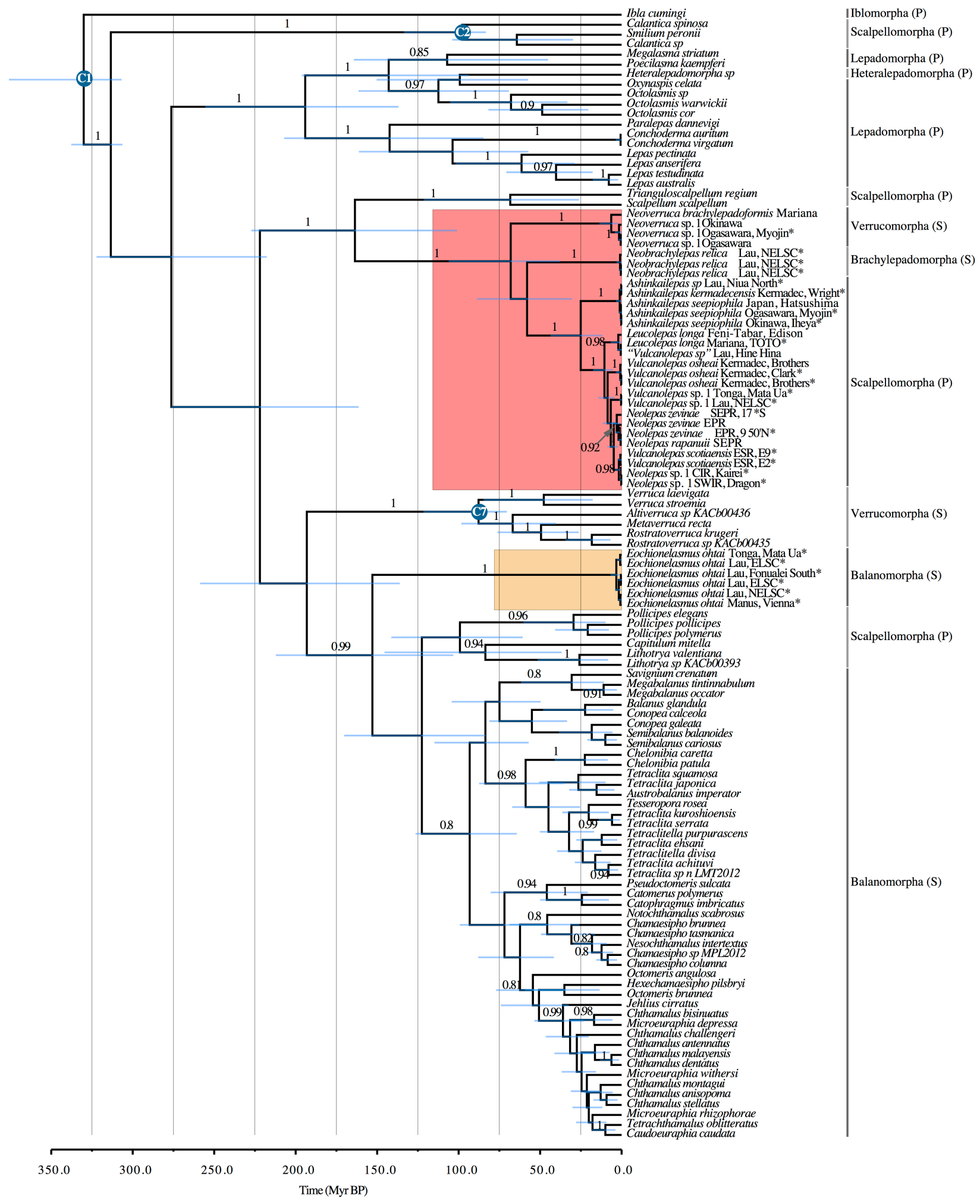

Figure 2. Maximum clade credibility ultrametric time-scaled tree, generated under the Birth-Death model tree prior, for the Thoracica-wide concatenated dataset. Red square indicates hydrothermal vent Clade A. Yellow square 
indicates hydrothermal vent Clade B. Node bars represent the $95 \%$ highest posterior density intervals. Branch labels show posterior probabilities. Blue circles in nodes indicate fossil calibration points as in (Pérez-Losada et al. 2008; Linse et al. 2013). Suborders belonging to the order Pedunculata (stalked or gooseneck barnacles) are indicated with (P). Suborders belonging to the order Sessilia (acorn barnacles) are indicated with (S). *Indicates data generated in this study.

\section{RAD-seq data quality control and loci clustering}

Sequence reads were de-multiplexed and quality filtered with the process_radtags program from the package Stacks v1.19 (Catchen et al. 2011; Catchen et al. 2013). Barcodes and Illumina adapters were excluded from each read and length was truncated to 90bp (-t 90) Reads with ambiguous bases were discarded (-c). Reads with an average quality score below 10 (-s 10) within a sliding window of $15 \%$ of the read length (-w 0.15) were discarded (-r). The rescue barcodes and RAD-tags algorithm was enabled (-r). Additional filtering, and the clustering within and between individuals to identify loci was performed using the program $p y$ RAD v2.01 (Eaton 2014). Reads with more than 33 bases with a quality score below 20 were also discarded. The minimum depth of coverage required to build a cluster was 5 (d 5 ). As in Hipp et al. (2014), three different clustering thresholds were explored (c 0.80, 0.85 and 0.90). Similarly,

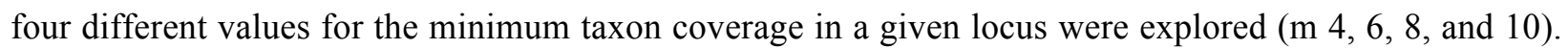
The maximum number of shared polymorphic sites in a locus was set to 3 (p 3). Loci were concatenated into combined RAD-seq matrices.

\section{RAD Phylogenetics}

Phylogenetic inferences of RAD-seq matrices, built with $p y$ RAD under each combination of clustering threshold and minimum taxon coverage parameters (as outlined above), were carried out in RAxMLHPC2 v8.0. We assumed a generalized time-reversible DNA substitution model with a gamma-distributed rate variation across sites. Branch support was assessed by 500 bootstrap replicates.

\section{RESULTS}

Complete Sanger-based marker datasets were obtained for all 94 individuals, except for 2 specimens of Vulcanolepas osheai. Sequences are stored in the GenBank database of the U.S. National Center for Biotechnology Information. Approximate sequence lengths for each marker were $700 \mathrm{bp}$ for $28 \mathrm{~S}, 657 \mathrm{bp}$ for coxI, and $327 \mathrm{bp}$ for $H 3$. Xia tests indicated substantial saturation at the Thoracica-wide level at third codon positions of coxI (Table S5). Little saturation was found in all other partitions. Maximum likelihood and Bayesian phylogenetic inferences from each Sanger-based marker produced mostly congruent trees that varied in the degree of resolution yet all showed poorly supported branches (i.e., 
posterior probability $<80$, bootstrap support $<80$ ) (supplementary electronic material). Analyses of the Thoracica-wide concatenated dataset generated a better-supported and -resolved phylogeny overall (Fig. 2, supplementary electronic material). The topologies of these trees were congruent with previously published phylogenetic hypotheses for the Thoracica (Pérez-Losada et al. 2008; Linse et al. 2013).

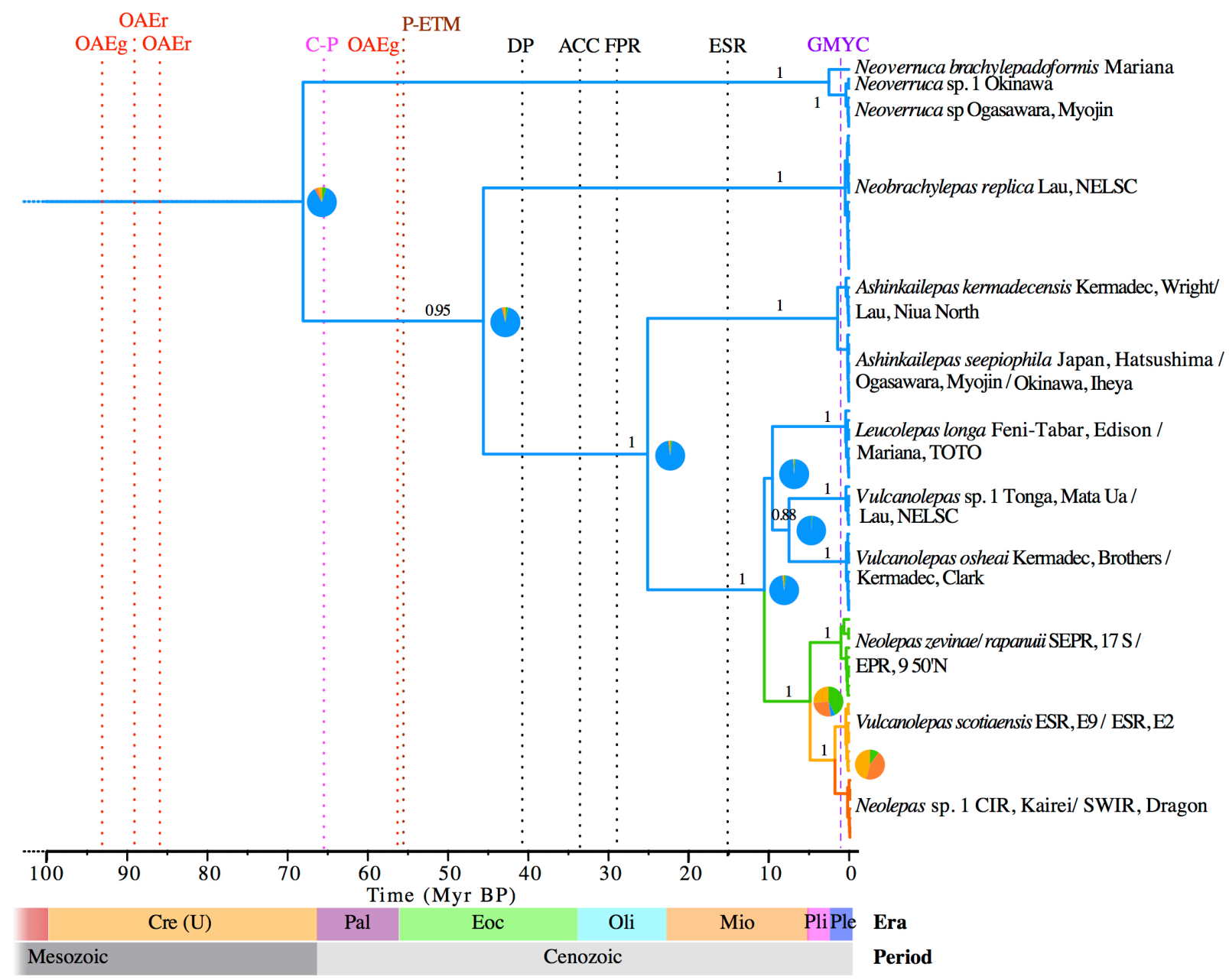

Figure 3. Clade A combined 28S, $H 3$, and coxI maximum clade credibility ultrametric time-scaled tree generated under the Birth-Death model. Branch colours show the most probable location states: western Pacific in blue, eastern Pacific in green, Southern Ocean south of the Atlantic in yellow and Indian Ocean in orange. Pie charts show the posterior probabilities of location states for each ancestral node (total pie area $=1$ ). Branch labels show posterior probabilities. Purple vertical dashed line indicates the maximum likelihood-inferred time for the speciationcoalescent threshold for species delimitation (GYMC). Vertical dotted lines indicate important events in geologic time: Oceanic Anoxic Events (red, OAEg for global and OAEr for regional), Cretaceous-Paleogene mass extinction 
(fuchsia, C-P), Paleocene-Eocene Thermal Maximum (brown, P-ETM), opening of the Drake Passage (black, DP), establishment of the Antarctic Circumpolar Current (black, ACC), disruption of the Farallon Pacific Ridge (black, FPR), formation of the East Scotia Rise (black, ESR). Geologic periods and eras are indicated with horizontal bars: upper Cretaceous (Cre (U)), Paleocene (Pal), Eocene (Eoc), Oligocene (Oli), Miocene (Mio), Pliocene (Pli), and Pleistocene (Ple).

RAD-seq datasets were obtained from 13 individuals representing the vent barnacle species in this study (Table S1). An average of 843,541 reads (SD 589,377) were obtained per individual. Reads are stored at the Sequence Read Archive (SRA) of NCBI. The great variability in sequencing yield was largely a product of varying of DNA integrity as some samples had notably degraded DNA (Table S6), as determined by agarose gel electrophoresis. An average of 712,306 reads per individual (SD 546,846), roughly $78 \%$ of all reads, were retained after quality filtering steps. In individuals with high-integrity DNA, the number of RAD-tag loci with depth of coverage greater than $4 \mathrm{X}$ was approximately 18,000, per individual. This number is congruent with the expected number of RAD-tags, between 10,000 and 30,000 , predicted for a barnacle, using the enzyme SbfI (Table S3). The average depth of coverage per locus was approximately $54 \mathrm{X}$ (SD $13 \mathrm{X}$ ). As expected, the number of loci per individual was higher as the clustering threshold was larger (Table S7). Phylogenetic trees obtained from the RAD-seq datasets were completely resolved, highly supported as indicated by bootstrap resampling, and were largely congruent with the trees produced with Sanger-based data.

\section{Phylogenetic Inferences}

Analyses of Sanger-based markers revealed that barnacles from deep-sea hydrothermal vents are divided into two well-supported (posterior probability = 1, bootstrap support > 99) main clades (Fig. 2): Clade A contains the genera Neobrachylepas Newman \& Yamaguchi, 1995 (Order Sessilia, Suborder Brachylepadomorpha), Neoverruca Newman, 1989 (Order Sessilia, Suborder Verrucomorpha), Ashinkailepas Yamaguchi el al. 2004 (Order Pedunculata, Suborder Scalpellomorpha), Leucolepas Southward \& Jones, 2003 (Suborder Scalpellomorpha), Vulcanolepas Southward \& Jones, 2003 (Suborder Scalpellomorpha), and Neolepas Newman, 1979 (Suborder Scalpellomorpha); and Clade B was restricted to the genus Eochionelasmus Yamaguchi, 1990 (Order Sessilia, Suborder Balanomorpha). Clade A is well supported as the sister taxon to the predominantly deep-sea clade of the Scalpellidae (Pérez-Losada et al. 2008; Linse et al. 2013). Clade B Eochionelasmus is associated with the paraphyletic Balanomorpha group, however the lack of support and resolution within the later group prevents an unambiguous phylogenetic placement. 

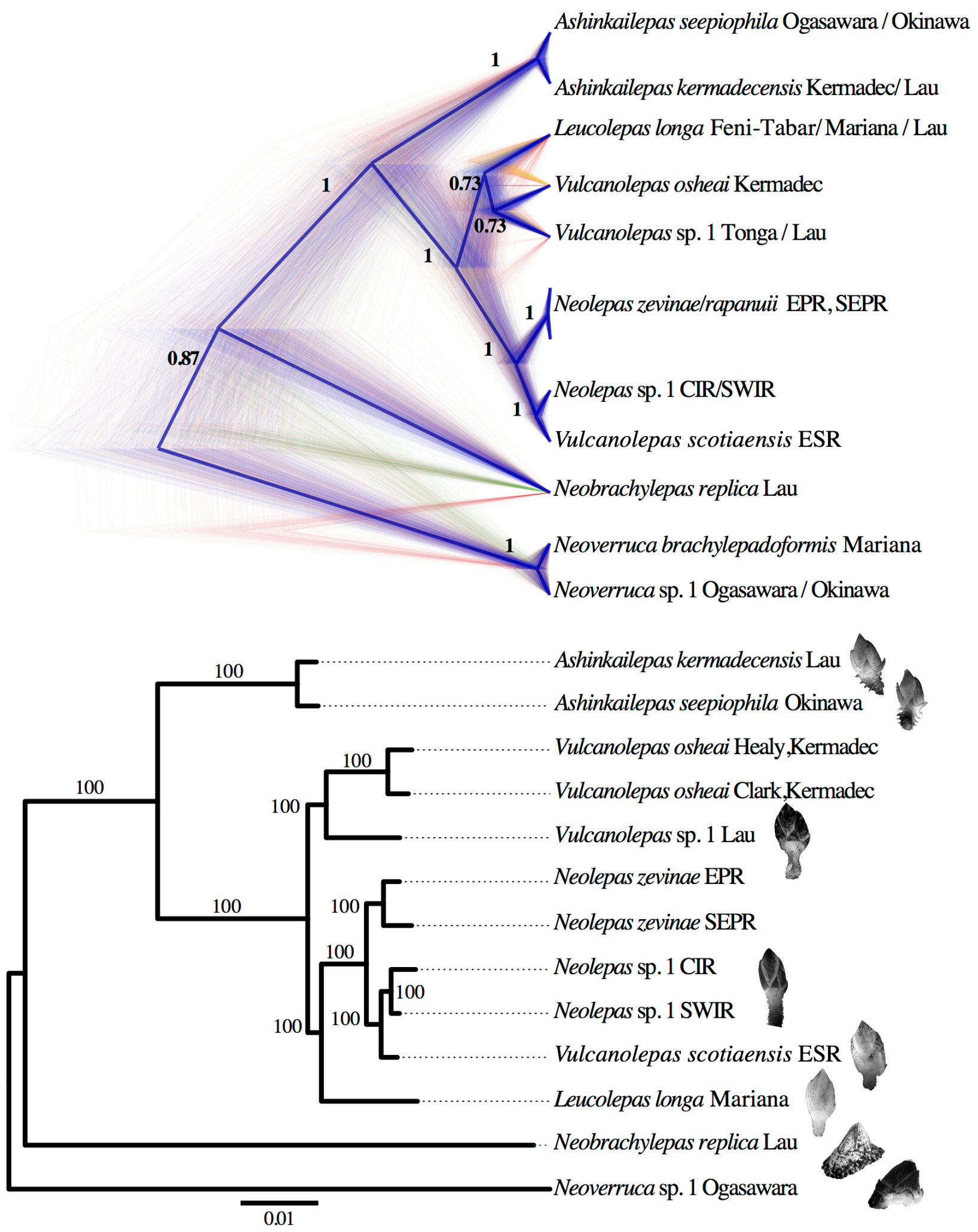

Figure 4. Top. Claudogram of the posterior distribution of species trees. High color density is indicative of areas in the species trees with high topology agreement. Different colors represent different topologies. The maximum clade 
credibility species tree is shown with thicker branches. Branch labels show posterior probabilities. Trees with the same topology as the maximum clade credibility species tree are coloured in blue. Trees with different topologies are colored yellow or red. Bottom. Maximum likelihood phylogenetic tree inferred with RAD-seq data. The matrix used for this tree was obtained with a clustering threshold of 0.85 and minimum taxon coverage of 6 . This matrix contains 828,960 nucleotide sites in 9,766 loci. 76,353 of the sites are variable and 26,955 are parsimony informative. This matrix contains $43.54 \%$ missing data. Branch labels show bootstrap support values. Scale bar indicates substitutions per site. Barnacle species images are from individuals included in this study. Species names are followed by the collection regions.

Neobrachylepas and Neoverruca appear as the extant representatives of the earliest divergent lineages in Clade A; however, their order of divergence is unclear due to lack of strong branch support. The rest of the genera in Clade A belong to the Family Eolepadidae. The genus Ashinkailepas belongs to the earliest divergent lineage in the family (Fig. 3), and contains two sub-clades, one grouping individuals from the Izu-Ogasawara (Bonin) Arc and the Okinawa Trough (identified as Ashinkailepas seepiophila), and the second grouping individuals from the Lau Basin, and the Kermadec Arc. The latter sub-clade includes a paratype of A. kermadecensis. Neither genus Vulcanolepas nor Neolepas is monophyletic. The Vulcanolepas/Leucolepas from the Kermadec Arc, Lau Basin, and Mariana Arc belong to lineages that appear to have diverged earlier in history with respect to a well-supported and well-resolved clade made up by $N$. zevinae/rapanuii from the East Pacific Rise and its sister sub-clade of $V$. scotiaensis from the East Scotia Ridge and Neolepas sp. 1 from the Southwest and Central Indian Ridge.

\section{Topological Hypothesis Testing}

None of the phylogenetic hypotheses inferred from the Thoracica-wide concatenated Sanger-based dataset support the monophyly of barnacles from deep-sea hydrothermal vents (Fig. 2). The topological test showed that the hypothesis of monophyly was significantly less probable than the hypothesis of nonmonophyly (marginal log-likelihoods -16928.21 and -16908.62 respectively). The large difference in loglikelihoods (> 5) (Kass \& Raftery 1995) constitutes strong contradictory evidence against the monophyly of vent barnacles as originally suggested by Pérez-Losada et al. (2008).

\section{Divergence Estimates and Biogeographic History}

Tree time calibrations of the combined Sanger-based dataset produced divergence estimates slightly older under the Yule tree prior of constant speciation, when compared with the nearly identical estimates obtained under the Birth-Death prior models (Fig. 2 and supplementary electronic material). These divergence estimates are consistent with estimates from Linse et al. (2013). The tree obtained under the Birth-Death model had the best likelihood score; however, no significant differences were encountered 
among models (log-likelihood $<1$ ). The time to the most recent common ancestor (TMRCA) of Clade A was estimated at 68.0 million years before present (Myr BP) (95\% Highest Posterior Density Interval [HPD]: 38.2-105.9) under the Birth-Death models (BD) and 79.3 Myr BP (95\% HPD: 47.1-121.5) under the Yule model of constant speciation rate. The TMRCAs of Eolepadidae and the NeolepasVulcanolepas-Leucolepas sub-clade were estimated at 25.1 Myr BP (95\% HPD: 12.1-43.3) and 10.5 Myr BP (95\% HPD: 5.4-17.3) under BD, and 31.2 Myr BP (95\% HPD: 15.4-53.7) and 13.8 Myr BP (95\% HPD: 7.5-23.1) under the Yule model, respectively. Divergence between Pacific and non-Pacific Neolepas-Vulcanolepas eolepadids was estimated to have occurred 4.8 Myr BP (95\% HPD: 2.3-8.5) and 6.4 Myr BP (95\% HPD: 3.0-11.2) under BD and Yule models, respectively. The split between the East Scotia Ridge and the Indian Ocean lineages occurred 1.7 Myr BP (95\% HPD: 0.4-3.8) under BD and 2.3 (95\% HPD: 0.5-4.9) under Yule. The TMRCA of Clade B Eochionelasmus was estimated at 3.2 Myr BP (95\% HPD: 1.1-6.7) under the Birth-Death model and 4.2 Myr BP (95\% HPD: 1.3-8.8) under Yule. The analysis of historical biogeography suggest with high-probability that hydrothermal vent barnacles from Clade A originated in the western Pacific, and during the late Miocene -Pliocene colonized the Eastern Pacific, the Southern Ocean south of the Atlantic, and the Indian Ocean (Fig. 3).

\section{Species Delimitation}

GMYC analyses of Clade A identified a transition point between interspecific speciation-extinction processes and intraspecific coalescent processes at 0.6 Myr BP for the time-scaled combined Sangerbased phylogeny estimated with the Birth-Death model tree prior (Fig. 3). The GMYC model showed a significant $(\alpha=0.05)$ better fit to the data than the null model of uniform coalescent branching rates (likelihood ratio $=25.9, \mathrm{p}<0.0001$ ). There were 12 distinct clusters identified, which largely corresponded to species already described or populations that were presumed to be new species. Genetic distances (coxI uncorrected distances) among individuals within clusters ranged between 0 and $0.9 \%$. Genetic distances among individuals from different clusters ranged between 2 and 17.8\% (except for the two Neolepas zevinae/rapanuii clusters whose maximum distance was $0.9 \%$ ). Similarly in Clade B Eochionelasmus the genetic distances among individuals ranged between 0 and $0.9 \%$.

\section{Species Tree Estimation}

The topology of the inferred Sanger-based species tree is fully congruent with the topology of the phylogenetic hypothesis obtained with the concatenated Sanger-based markers dataset, and the branch support values are mostly equal (Fig. 4). Poorly-resolved regions of the tree include the relationships among lineages of Vulcanolepas/Leucolepas from the Kermadec Arc, Lau Basin, Mariana Trough, and basally the positions of Neoverruca and Neobrachylepas within Clade A. 


\section{RAD Phylogenetics}

RAD-seq matrices resulting from the three explored clustering thresholds (c 0.80, 0.85 and 0.90 ) contained similar numbers of loci and similar percentages of missing data per clustering parameter value used for the minimum taxon coverage in a given locus (approximately 15,500, 9,600, 3,800, and 600 loci, and $52 \%, 44 \%, 33 \%$ and $21 \%$ missing data, for $\mathrm{m} 4,6,8$, and 10 respectively; see Table S8 for details). The percentages of variable sites and parsimony informative sites across matrices ranged between 6.81 $13.18 \%$ and $2.26-4.22 \%$ respectively, being higher with smaller values of clustering thresholds and larger values of minimum taxon coverage. The tree topologies obtained from phylogenetic inferences of each matrix were identical to each other (supplementary electronic material). These topologies from RAD-seq matrices were also similar to the species tree obtained with Sanger-based markers (Fig. 4), only differing in the position of Leucolepas, appearing in the RAD-based trees as sister to the clade made up by N. zevinae/rapanuii from the East Pacific Rise, V. scotiaensis from the East Scotia Ridge and Neolepas sp. 1 from the Southwest and Central Indian Ridge. RAD-based trees topologies were highly supported with bootstrap values of 100 for all branches, except for the ones from matrices generated with a minimum taxon coverage parameter of $\mathrm{m} 10$. In these cases, the branches supporting the clades of Vulcanolepas from the Lau Basin and the Kermadec Arc, and of Leucolepas-N. zevinae/rapanuii-V. scotiaensis- Neolepas sp. 1 have bootstrap support values greater than 94 and 71, respectively.

\section{DISCUSSION}

\section{Are vent barnacles monophyletic?}

The inferred evolutionary history of hydrothermal vent barnacles is not consistent with the hypothesis of monophyly (single ancestry) as proposed by the smaller taxon-sampling studies of Pérez-Losada et al. (2008) and Linse et al. (2013), which included only two of the four families of vent barnacles. Our analyses of a significantly expanded dataset indicate that there are two main clades (Clade A and Clade B) (Fig. 2), thus suggesting that barnacles have colonized deep-sea hydrothermal vents at least twice in history. The results from a concurrent study by Perez-Losada et al. (2014) provide support to this inference by placing Clade B (Eochionelasmus ohtai) nested within the balanomorph barnacles, although the hypothesis of monophyly of vent barnacles was not explicitly tested in that study.

Deep-sea hydrothermal vent barnacle Clade A is the more diverse of the two, containing six of the seven genera included in this study. This clade also contains a remarkable diversity of morphologies, including asymmetric (Neoverrucidae) and symmetric (Neobrachylepadidae), pedunculate (Eolepadidae) and sessile (Neoverrucidae and Neobrachylepadidae) forms (Fig. 4) (note that neoverrucid barnacles have a 
pedunculated stage during early ontogenesis (Newman \& Hessler 1989)). The sister relationship of Clade A and the deep-sea pedunculate Scalpellidae (Fig. 2) (Pérez-Losada et al. 2008; Linse et al. 2013) suggests that the sessile state of the Neoverrucidae and Neobrachylepadidae is a derived state. This observation is consistent with the mounting evidence that the characters used to define higher taxonomic groups in Cirripedia need to be revised in light of multilocus molecular phylogenetic hypotheses (PérezLosada et al. 2008; Linse et al. 2013). A noteworthy example of this taxonomic and phylogenetic incongruence is the phylogenetic placement of $N$. relica nested in Clade A. N. relica is the sole living brachilepadoform species and until now was considered the most 'primitive' lineage of sessilian barnacles (Newman \& Yamaguchi 1995). Clade B only contains the genus Eochionelasmus. Despite its morphological and phylogenetic affinities with the Balanomorpha, the phylogenetic position of Eochionelasmus is this study is unstable. Similarly, Perez-Losada et al. (2014) found low support for the branches resolving the position Eochionelasmus ohtai within the balanomorphs. This instability is likely caused by the long branch supporting this clade, which may indicate a rapid evolutionary rate, old divergence, or taxonomic undersampling (Fig. 2, supplementary electronic material). Further taxonomic sampling of related genera and careful review of character use for systematics should help resolve its systematics.

Deep-sea hydrothermal vent environments have been characterized as being patchy and ephemeral habitats with extreme spatial and temporal gradients of temperature, reduced chemicals, oxygen and food supply (Van Dover 2000). These conditions present significant physiological and ecological challenges to organisms and act as environmental filters that promote the evolution and distribution of species with specialized adaptations (Tunnicliffe et al. 2003; Fisher et al. 2007). The widespread persistence of vent chemosynthetic environments throughout earth's geologic history (Shock et al. 1995) has likely been an important factor enabling the independent colonization by multiple lineages of barnacles, as well as of other taxa, e.g., mussels (Lorion et al. 2013) and decapods (Yang et al. 2013). Clade A is nested within a predominantly deep-sea clade Linse et al. (2013), suggesting a colonization of hydrothermal vents at depth. The nested position within Clade A of A. seepiophila - the only barnacle species known to live in both cold-seep and hydrothermal vent environments - indicates a single colonization of seep environments by vent ancestors. This pattern contrasts with the stepwise colonization scenario of deep-sea chemosynthetic environments, starting in organic substrates or cold-seeps and then moving to hydrothermal vents, as suggested for other taxonomic groups, e.g., mussels (Lorion et al. 2013). 


\section{Historical Biogeography}

The most common recent ancestor of hydrothermal-vent barnacles from Clade A likely lived in the late Mesozoic or early Cenozoic. The time to the most recent common ancestor inferred in this study is consistent with the timing inferred by Linse et al. (2013), but contrasts with the lower Cretaceous origin proposed by Pérez-Losada et al. (2008) and with classic hypotheses of antiquity of vent taxa, which proposed that hydrothermal-vent barnacles were mid-Mesozoic relict taxa (Newman 1979; Newman 1985). The discrepancy with the results from Pérez-Losada et al. (2008) is due to the exclusion of fossil calibration points because of uncertainty in the phylogenetic placement as described by Linse et al. (2013). The timing of radiation of Clade A during the Cenozoic is comparable to the estimates of origin and radiation in other chemosynthetic taxa, e.g., radiation of bresiliid shrimp 6.7-11.7 Myr BP (Shank et al. 1999); origin of siboglinid tubeworms ca. $60 \mathrm{Myr}$ BP (Chevaldonne et al. 2002); radiation of chemosynthetic mussels at ca. 45 Myr BP (Lorion et al. 2013); radiation of kiwaid yeti crabs starting at ca. 30 Myr BP; also see reviews by Little and Vrijenhoek (2003) and Vrijenhoek (2013). A recent origin and radiation of most modern vent taxa and many other deep-sea taxa (Little \& Vrijenhoek 2003; Smith \& Stockley 2005; Strugnell et al. 2008) is consistent with the inference of a major deep-sea mass extinction event during the Cretaceous-Paleogene period boundary (Raup \& Sepkoski 1982; Horne 1999; Harnik et al. 2012) (see Fig. 3). Several smaller-scale extinction events linked to regional Oceanic Anoxic Events, ocean acidification and temperature changes also occurred during the Cretaceous period and at the Paleocene-Eocene epoch boundary (Jacobs \& Lindberg 1998; Rogers 2000; Harnik et al. 2012).

The most probable place of origin of the modern vent barnacle lineage from Clade A is the western Pacific, as indicated and highly supported by Bayesian ancestral state reconstruction. This is also the region where the oldest lineages and the highest diversity are found. The heterogeneity of depths in hydrothermal vent systems in the western Pacific, and the close proximity to other chemosynthetic ecosystems such as cold seeps and organic enrichments, both shallow and deep, have been suggested as important factors driving the re-colonization of vent environments and subsequent diversification (Moalic et al. 2011). Our analyses suggest that the most probable path of dispersal out of the western Pacific was a migration eastward during the Miocene epoch, possibly following hydrothermal vent habitats along the Pacific-Antarctic Ridge, and colonization of the eastern Pacific. The neolepadids from the East Pacific

Rise have a coalescence point that is posterior to the Oligocene disruption of the Pacific-Farallon Ridge by subduction under the North American Plate, ca. 28.5 Myr BP (Fig. 3) (Atwater 1989), which can explain why barnacles are absent from the north-eastern Pacific vents along the Juan de Fuca Ridge. A spreading through the southern hemisphere likely followed to the East Scotia Ridge and South Sandwich Arc during the late Miocene epoch, reaching the Southwest Indian Ridge and Central Indian Ridge during 
the Pliocene/Pleistocene epochs. No vent barnacle species have been found at Mid Atlantic Ridge hydrothermal vents, although the southern portion of this major mid-ocean ridge remains largely unexplored.

The proposed history of dispersal is congruent with the timing of opening of the Drake Passage during the mid Eocene epoch, ca. 41 Myr BP (Scher 2006), the late Eocene establishment of the eastward-flowing Antarctic Circumpolar Current (ACC), ca. 34 Myr BP (Scher 2006), and the mid Miocene formation of the East Scotia Rise, ca. 15 Myr BP (Livermore 2003) (see Fig. 3). Hydrothermal vent yeti crabs (Decapoda: Anomura: Kiwaidae) share an almost identical pattern of historical dispersal from the eastern Pacific to the East Scotia Ridge and the Southwest Indian Ridge (see Roterman et al. (2013) for a detailed hypothesis of vicariance in this group). A likely origin in the western or northwestern Pacific followed by migration and colonization eastward throughout the southern hemisphere during the Miocene epoch has also been inferred for other non-vent deep-sea taxa such as the octocoral Paragorgia arborea (Herrera et al. 2012), and other marine taxa such as the spiny dogfish Squalus acanthias (Verissimo et al. 2010) and the bryozoan Membranipora membranacea (Schwaninger 2008). These observations provide support for the biogeographic hypothesis proposed by Moalic et al. (2011) that the western Pacific was a centre of origin of modern vent fauna from which most taxa dispersed globally. However, our data do not support the idea of direct links between the western Pacific communities and the Indian Ocean, but rather a stepping-stone mode of dispersal in the southern hemisphere following the direction of the dominant ACC. We suggest that the geological processes and dispersal mechanisms discussed here can explain the current distribution patterns of many other marine taxa and have played an important role shaping extant deep-sea faunal diversity

The history of Clade B is not well resolved. The phylogenetic hypothesis here presented suggests that the divergence of this lineage within the Balanomorpha occurred in the Mesozoic era (Fig. 2). However this inferred antiquity is likely to be an artifact caused by taxonomic undersampling in this group. Additional data from other Echionelasmus populations, e.g., E. paquensis from the eastern Pacific, as well as from confamilial species and related groups would provide greater resolution of the evolution of Clade B.

\section{Species Delimitation and Relationships}

Inferences of species boundaries in Clade A, based on the generalized mixed Yule-coalescent method, are largely congruent with descriptions of putative morphospecies. The identified species clusters are wellconstrained geographically by mid-ocean spreading ridge system and neighboring island arc basins (Figs. 3 and 4). Divergences among congeners in Ashinkailepas and Neoverruca are largely consistent with the 
biogeographic boundary between the northwest and southwest Pacific, inclusive of the Mariana arc, proposed by Bachraty et al. (2009). Relationships among Vulcanolepas, Leucolepas and Neolepas species clusters remain contentious due to the non-monophyly of all three genera as defined by Buckeridge et al. (2013) and thus require substantial revision.

There is a lack of overlap in genetic distances for the coxI barcode marker within and among inferred species clusters. The maximum genetic distance within species clusters of $0.9 \%$, and the minimum distance among species clusters of $2 \%$ are consistent with the proposed threshold value of $c a .2 \%$ to define species boundaries through DNA barcoding in Crustacea (Hebert et al. 2003; Lefebure et al. 2006). Similarly, the maximum genetic distance among individuals of Echionelasmus ohtai is $0.9 \%$. The only exception to this pattern is found in the Neolepas zevinae/rapanuii species cluster pair, where the maximum distance between clusters is $0.9 \%$. There is no phylogenetic support for this split or geographic segregation between specimens from the East Pacific Rise and southern East Pacific Rise, thus suggesting that the division of Neolepas zevinae/rapanuii is not indicative of species-level differentiation. The barcoding gap within and among species has been consistently found in other barnacle taxa (Tsang et al. 2008; Tsang et al. 2009; Yoshida et al. 2011) and in crustaceans in general (Costa et al. 2007; Matzen da Silva et al. 2011), thus our coxI genetic distance data provides further support to the species delimitations proposed for Clade A. The species delimitation framework developed will enable rapid species assignments as specimens from newly explored geographical regions become available.

\section{RAD phylogenetics}

Several sources of uncertainty have been associated with the use of the few traditional sequence markers available for non-model organisms (e.g., mitochondrial and ribosomal genes), including low variability, biased loci sampling, poor genomic representation, low statistical power, and inclusion of pseudogenes, among others. The effects of these are often hard to identify due to the paucity of multi-locus genomewide comparative datasets. Such problems have been recognized and accounted for in model organisms by comparing large numbers of genomic DNA sequences from various individuals and identifying thousands of variable regions across the genome (Rokas et al. 2003; Clark et al. 2007). Recent technological and methodological developments in next-generation sequencing platforms and methodologies, such as RAD-seq, have made genomic resources increasingly accessible and available for phylogenetics in non-model organisms (Wagner et al. 2012; Eaton \& Ree 2013; Jones et al. 2013; Reitzel et al.2013), thus offering a great opportunity to overcome the difficulties inherent to the use of traditional approaches in many taxa. 
In this study, we demonstrated that RAD-seq data provide strong support for the overall evolutionary history of vent barnacles inferred with traditional Sanger-based markers, and allow the inference of a fully resolved and supported phylogenetic tree. The small difference in topology between the species tree inferred with Sanger-based markers and the RAD-seq trees does not alter any of the conclusions regarding the biogeographical history or species delimitation of vent barnacles, but does have taxonomic implications. Further sampling and a follow-up morphological taxonomic revision would be needed to clarify the validity of the currently described genera. This study demonstrates the utility of comparative Sanger-based and RAD sequencing as a means of comparative phylogenetic inference validation in poorly known taxa such as deep-sea invertebrates.

\section{CONCLUSIONS}

Phylogenetic inferences and topology tests indicate that hydrothermal vent barnacles are not a monophyletic group. The likely timing of barnacle radiation in hydrothermal vent ecosystems was during the late Cenozoic, consistent with the timing of other specific deep-sea taxa, and correlated to the occurrence of major extinction events. Our analyses suggest that the western Pacific was the place of origin of the major hydrothermal vent barnacle lineage, followed by circumglobal colonization eastward along the southern hemisphere during the Neogene period. Inferences of species boundaries based on generalized mixed Yule-coalescent methods and DNA barcoding are largely congruent with morphological descriptions of putative species. RAD-seq data provide strong support for the overall evolutionary history inferred from Sanger-based markers and a fully resolved backbone of the vent barnacle phylogenetic tree. These results also constitute critical baseline data with which to assess potential effects of anthropogenic disturbances on deep-sea ecosystems.

\section{AUTHOR CONTRIBUTIONS}

SH and TMS conceived and designed research. SH performed research. SH analyzed data. SH, TMS and HW collected and contributed samples and reagents. SH wrote the paper with comments from TMS and HW. 


\section{DATA ACCESSIBILITY}

\section{Supplementary electronic material}

Nucleotide alignments, input files, are tree files are available in Dryad DOI: doi:10.5061/dryad.7kn5k

\section{Raw data}

Raw DNA sequences are available at the U.S. National Center for Biotechnology Information (NCBI) GenBank and Sequence Read Archive (SRA accession number SRP051026).

\section{ACKNOWLEDGEMENTS}

This research was supported by the Office of Ocean Exploration and Research of the National Oceanic and Atmospheric Administration (NA09OAR4320129 to TS); the Division of Ocean Sciences of the National Science Foundation (OCE-1131620 to TS); the Division of Polar Programs of the National Science Foundation (PLR-0739675 to TS); the Astrobiology Science and Technology for Exploring Planets program of the National Aeronautics and Space Administration (NNX09AB76G to TS); and the Academic Programs Office (Ocean Ventures Fund to SH), the Ocean Exploration Institute (Fellowship support to TMS) and the Ocean Life Institute of the Woods Hole Oceanographic Institution (internal grant to TMS and SH). For enabling access to key specimens we thank K. Iizasa (U. Tokyo), Y. Suzuki (U. Tokyo), S. Nakagawa (JAMSTEC), P. Tyler (NOCS), J. Copley (NOCS), A. Rogers (Oxford), N. Roterman (Oxford), M. Clark (NIWA), A. Rowden (NIWA), K. Schnabel (NIWA), S. Mills (NIWA), J. Resing (NOAA-PMEL), R. Embley (NOAA-PMEL), A. Reysenbach (PSU), M.K. Tivey (WHOI), P. Fryer (UH), C. Langmuir (Harvard), K. Von Damm (UNH), M. Lilley (UW), the masters, crew, scientific personnel, and funding agencies of expeditions AT03-28, AT07-06, JC042, JC067, KM0417, KM0912, KOK0505, KOK0506, NT97-10, NT97-14, NT99-09, RR1211, TAN1007, TAN1104, TAN1206, TN234, TN236, YK06-13, and YK09-13. Specimens provided by the National Institute of Water and Atmospheric Research (NIWA) were collected under research programs: Kermadec Arc Minerals, funded by the New Zealand Ministry of Business, Innovation \& Employment (MBIE), Auckland University, Institute of Geological and Nuclear Science (GNS), and WHOI; Ocean Survey 20/20 funded by Land Information New Zealand; Impact of resource use on vulnerable deep-sea communities (CO1X0906), funded by MBIE; and the Joint New Zealand-USA 2005 NOAA Ring of Fire Expedition, part of NIWA's Seamount Program (FRST CO1X0508). We thank A. Tarrant, A. Reitzel, and three anonymous reviewers, for providing helpful comments that improved this manuscript. 


\section{REFERENCES}

Atwater T (1989) Plate tectonic history of the northeast Pacific and western North America. In: The Eastern Pacific Ocean and Hawaii (eds. Winterer EL, Hussong DM, Decker RW), pp. 21-72. Geological Society of America, Boulder, Colorado.

Bachraty C, Legrende P, Desbruyeres D (2009) Biogeographic relationships among hydrothermal vent faunas on a global scale. Deep-Sea Research Part I-Oceanographic Research Papers 56, 13711378.

Baird N, Etter P, Atwood T, et al. (2008) Rapid SNP discovery and genetic mapping using sequenced RAD markers. PLoS One 3, e3376.

Baker ET, German CR (2004) On the global distribution of hydrothermal vent fields. Mid-Ocean Ridges: Hydrothermal Interactions between the Lithosphere and Oceans 148, 245-266.

Bensasson D, Zhang D, Hartl D, Hewitt G (2001) Mitochondrial pseudogenes: evolution's misplaced witnesses. Trends in Ecology \& Evolution 16, 314-321.

Boschen RE, Rowden AA, Clark MR, Gardner JPA (2013) Mining of deep-sea seafloor massive sulfides: A review of the deposits, their benthic communities, impacts from mining, regulatory frameworks and management strategies. Ocean and Coastal Management 84, 54-67.

Brito PH, Edwards SV (2008) Multilocus phylogeography and phylogenetics using sequence-based markers. Genetica 135, 439-455.

Buckeridge J (2000) Neolepas osheai sp. nov., a new deep-sea vent barnacle (Cirripedia: Pedunculata) from the Brothers Caldera, south-west Pacific Ocean. New Zealand Journal of Marine and Freshwater Research 34, 409-418.

Buckeridge JS, Linse K, Jackson JA (2013) Vulcanolepas scotiaensis sp. nov., a new deep-sea scalpelliform

barnacle (Eolepadidae: Neolepadinae) from hydrothermal vents in the Scotia Sea, Antarctica. Zootaxa 3745, 551-568.

Cairns S (2007) Deep-water corals: an overview with special reference to diversity and distribution of deep-water scleractinian corals. Bulletin of Marine Science 81, 311-322.

Carpenter KE, Barber PH, Crandall ED, et al. (2011) Comparative phylogeography of the Coral Triangle and implications for marine management. Journal of Marine Biology 2011, 1-14.

Catchen J, Hohenlohe PA, Bassham S, Amores A, Cresko WA (2013) Stacks: an analysis tool set for population genomics. Molecular Ecology 22, 3124-3140.

Catchen JM, Amores A, Hohenlohe P, Cresko W, Postlethwait JH (2011) Stacks: building and genotyping Loci de novo from short-read sequences. G3 1, 171-182.

Chevaldonne P, Jollivet D, Desbruyeres D, Lutz RA, Vrijenhoek RC (2002) Sister-species of eastern Pacific hydrothermal vent worms (Ampharetidae, Alvinellidae, Vestimentifera) provide new mitochondrial COI clock calibration. Cahiers De Biologie Marine 43, 367-370.

Clark AG, Eisen MB, Smith DR, et al. (2007) Evolution of genes and genomes on the Drosophila phylogeny. Nature 450, 203-218.

Colgan DJ, McLauchlan A, Wilson GDF, et al. (1998) Histone H3 and U2 snRNA DNA sequences and arthropod molecular evolution. Australian Journal of Zoology 46, 419-437.

Costa FO, deWaard JR, Boutillier J, et al. (2007) Biological identifications through DNA barcodes: the case of the Crustacea. Canadian Journal of Fisheries and Aquatic Sciences 64, 272-295.

Cubelio SS, Tsuchida S, Watanabe S (2007) Vent associated Munidopsis (Decapoda : Anomura : Galatheidae) from Brothers Seamount, Kermadec Arc, Southwest Pacific, with description of one new species. Journal of Crustacean Biology 27, 513-519.

Darriba D, Taboada GL, Doallo R, Posada D (2012) jModelTest 2: more models, new heuristics and parallel computing. Nat Methods $\mathbf{9}, 772$. 
Desbruyeres D, Alayse-Danet A-M, Ohta S, the Scientific Parties of biolauand s (1994) Deep-sea hydrothermal communities in Southwestern Pacific back-arc basins (the North Fiji and Lau Basins): Composition, microdistribution and food web. Marine Geology 116, 227-242.

Drummond A, B A, S B, et al. (2011) Geneious v5.4. Available from http://www.geneious.com/.

Drummond AJ, Suchard MA, Xie D, Rambaut A (2012) Bayesian phylogenetics with BEAUti and the BEAST 1.7. Molecular Biology and Evolution 29, 1969-1973.

Eaton DA (2014) PyRAD: assembly of de novo RADseq loci for phylogenetic analyses. Bioinformatics 30, 1844-1849.

Eaton DAR, Ree RH (2013) Inferring phylogeny and introgression using RADseq data: An example from flowering plants (Pedicularis: Orobanchaceae). Systematic Biology 62, 689-706.

Edwards SV (2008) Is a new and general theory of molecular systematics emerging? Evolution 63, 1-19.

Fan Y, Wu R, Chen MH, Kuo L, Lewis PO (2011) Choosing among partition models in Bayesian phylogenetics. Molecular Biology and Evolution 28, 523-532.

Fisher CR, Takai K, Le Bris N (2007) Hydrothermal vent ecosystems. Oceanography 20, 14-23.

Folmer O, Black M, Hoeh W, Lutz R, Vrijenhoek R (1994) DNA primers for amplification of mitochondrial cytochrome c oxidase subunit I from diverse metazoan invertebrates. Molecular Marine Biology and Biotechnology 3, 294-299.

Fujisawa T, Barraclough TG (2013) Delimiting species using single-locus data and the Generalized Mixed Yule Coalescent approach: A revised method and evaluation on simulated data sets. Systematic Biology 62, 707-724.

Gernhard T (2008) The conditioned reconstructed process. Journal of Theoretical Biology 253, 769-778.

Harnik PG, Lotze HK, Anderson SC, et al. (2012) Extinctions in ancient and modern seas. Trends in Ecology \& Evolution 27, 608-617.

Hebert PDN, Cywinska A, Ball SL, deWaard JR (2003) Biological identifications through DNA barcodes. Proceedings of the Royal Society B: Biological Sciences 270, 313-321.

Heled J, Drummond AJ (2010) Bayesian inference of species trees from multilocus data. Molecular Biology and Evolution 27, 570-580.

Herrera S, Munro C, Nganro N, et al. (2010) Biodiversity of the deep-sea benthic fauna in the SangiheTalaud region, Indonesia: Observations from the INDEX-SATAL 2010 expedition. AGU Fall Meeting Abstracts 1, 1234.

Herrera S, Reyes-Herrera PH, Shank TM (Chapter 2) Genome-wide predictability of restriction sites across the eukaryotic tree of life.

Herrera S, Shank TM, Sanchez JA (2012) Spatial and temporal patterns of genetic variation in the widespread antitropical deep-sea coral Paragorgia arborea. Molecular Ecology 21, 6053-6067.

Hessler RR, Lonsdale PF (1991) Biogeography of Mariana Trough hydrothermal vent communities. Deep-Sea Research Part a-Oceanographic Research Papers 38, 185-199.

Hipp AL, Eaton DAR, Cavender-Bares J, et al. (2014) A framework phylogeny of the American Oak clade based on sequenced RAD data. PLoS One 9, e93975.

Horne DJ (1999) Ocean circulation modes of the phanerozoic: implications for the antiquity of deep-sea benthonic invertebrates. Crustaceana 72, 999-1018.

Jacobs DK, Lindberg DR (1998) Oxygen and evolutionary patterns in the sea: Onshore /offshore trends and recent recruitment of deep-sea faunas. Proceedings Of The National Academy Of Sciences Of The United States Of America 95, 9396-9401.

Jones DS (1993) A new Neolepas (Cirripedia: Thoracica: Scalpellidae) from an abyssal hydrothermal vent, southeast Pacific. Bulletin of Marine Science 52, 937.

Jones JC, Fan S, Franchini P, Schartl M, Meyer A (2013) The evolutionary history of Xiphophorus fish and their sexually selected sword: a genome-wide approach using restriction site-associated DNA sequencing. Molecular Ecology 22, 2986-3001.

Kass RE, Raftery AE (1995) Bayes factors. Journal of the American Statistical Association 90, 773-795.

Katoh K, Misawa K, Kuma K, Miyata T (2002) MAFFT: a novel method for rapid multiple sequence alignment based on fast Fourier transform. Nucleic Acids Research 30, 3059-3066. 
Lefebure T, Douady CJ, Gouy M, Gilbert J (2006) Relationship between morphological taxonomy and molecular divergence within Crustacea: Proposal of a molecular threshold to help species delimitation. Molecular Phylogenetics and Evolution 40, 435-447.

Lemey P, Rambaut A, Drummond AJ, Suchard MA (2009) Bayesian phylogeography finds its roots. PLoS Computational Biology 5, e1000520.

Linse K, Jackson JA, Fitzcharles E, Sands CJ, Buckeridge JS (2013) Phylogenetic position of Antarctic Scalpelliformes (Crustacea: Cirripedia: Thoracica). Deep-Sea Research Part I-Oceanographic Research Papers 73, 99-116.

Little CTS, Vrijenhoek RC (2003) Are hydrothermal vent animals living fossils? Trends in Ecology \&amp;amp; Evolution 18, 582-588.

Livermore R (2003) Back-arc spreading and mantle flow in the East Scotia Sea. In: Intra-oceanic subduction systems: tectonic and magmatic processes, pp. 315-331. Geological Society of London Special Publications.

Lopez JV, Yuhki N, Masuda R, Modi W, O'Brien SJ (1994) Numt, a recent transfer and tandem amplification of mitochondrial DNA to the nuclear genome of the domestic cat. Journal of Molecular Evolution 39, 174-190.

Lorion J, Kiel S, Faure B, et al. (2013) Adaptive radiation of chemosymbiotic deep-sea mussels. Proceedings of the Royal Society B: Biological Sciences 280, 20131243-20131243.

Marsh L, Copley JT, Huvenne VAI, et al. (2012) Microdistribution of faunal assemblages at deep-sea hydrothermal Vents in the Southern Ocean. PLoS One 7, e48348.

Matzen da Silva J, Creer S, dos Santos A, et al. (2011) Systematic and evolutionary insights derived from mtDNA COI barcode diversity in the Decapoda (Crustacea: Malacostraca). PLoS One 6, e19449.

McClain C, Mincks SL (2010) The dynamics of biogeographic ranges in the deep sea. Proceedings of the Royal Society B: Biological Sciences 277, 3533-3546.

Moalic Y, Desbruyeres D, Duarte CM, et al. (2011) Biogeography revisited with network theory: retracing the history of hydrothermal vent communities. Systematic Biology 61, 127-137.

Monaghan MT, Wild R, Elliot M, et al. (2009) Accelerated species inventory on madagascar using coalescent-based models of species delineation. Systematic Biology 58, 298-311.

Nakamura K, Watanabe H, Miyazaki J, et al. (2012) Discovery of new hydrothermal activity and chemosynthetic fauna on the Central Indian Ridge at 18 degrees -20 degrees S. PLoS One 7, e32965.

Newman WA (1979) A new scalpellid (Cirripedia): a Mesozoic relic living near an abyssal hydrothermal spring. Transactions of the San Diego Society of Natural History 19, 153-167.

Newman WA (1985) The abyssal hydrothermal vent invertebrate fauna. A glimpse of antiquity? Bulletin of the Biological Society of Washington 6, 231-242.

Newman WA, Hessler RR (1989) A new abyssal hydrothermal verrucomorphan (Cirripedia; Sessilia): the most primitive living sessile barnacle. Transactions of the San Diego Society of Natural History 21, 259-273.

Newman WA, Yamaguchi T (1995) A new sessile barnacle (Cirripedia, Brachylepadomorpha) from the Lau Back-Arc Basin, Tonga; first record of a living representative since the Miocene. Bulletin du Muséum national d'Histoire Naturelle. Section A, Zoologie, Biologie et Ecologie Animales 17, 221-243.

Newman WA, Yamaguchi T, Southward AJ, Segonzac M (2006) Arthropoda, Crustacea, Cirripedia. In: Handbook of Deep-Sea Hydrothermal Vent Fauna (eds. Desbruyeres D, Segonzac M, Bright M), pp. 356-357. Denisia.

Nylander JA, Wilgenbusch JC, Warren DL, Swofford DL (2008) AWTY (are we there yet?): a system for graphical exploration of MCMC convergence in Bayesian phylogenetics. Bioinfomatics 24, 581583.

Ohno T, Fujikura K, Hashimoto J, Fiujiwara Y, Segawa S (1996) The Hydrothermal Vent Community at the Kaikata Seamount near Ogasawara (Bonin) Islands, South Japan, pp. 221-229. (JAMSTEC) / Japan Marine Science and Technology Center (JAMSTEC). 
Ohta S (1990) Deep-sea submersible survey of the hydrothermal vent community on the northeastern slope of the Iheya ridge, the Okinawa Trough. In: The Sixth Symposium on Deep-Sea Research, pp. 145-156. (JAMSTEC) / Japan Marine Science and Technology Center (JAMSTEC).

Pérez-Losada M, Harp M, Høeg JT, et al. (2008) The tempo and mode of barnacle evolution. Molecular Phylogenetics and Evolution 46, 328-346.

Perez-Losada M, Hoeg JT, Simon-Blecher N, et al. (2014) Molecular phylogeny, systematics and morphological evolution of the acorn barnacles (Thoracica: Sessilia: Balanomorpha). Mol Phylogenet Evol 81, 147-158.

Pons J, Barraclough TG, Gomez-Zurita J, et al. (2006) Sequence-based species delimitation for the DNA taxonomy of undescribed insects. Systematic Biology 55, 595-609.

Rambaut A, Drummond AJ (2007) Tracer v1.4, Available from http://beast.bio.ed.ac.uk/Tracer.

Raup DM, Sepkoski JJJ (1982) Mass extinctions in the marine fossil record. Science 215, 1501-1503.

Reid WDK, Sweeting CJ, Wigham BD, et al. (2013) Spatial differences in east scotia ridge hydrothermal vent food webs: influences of chemistry, microbiology and predation on trophodynamics. PLoS One 8, e65553.

Reitzel AM, Herrera S, Layden MJ, Martindale MQ, Shank TM (2013) Going where traditional markers have not gone before: utility of and promise for RAD sequencing in marine invertebrate phylogeography and population genomics. Molecular Ecology 22, 2953-2970.

Rogers AD (2000) The role of the oceanic oxygen minima in generating biodiversity in the deep sea. Deep-Sea Research Part II 47, 119-148.

Rogers AD, Tyler PA, Connelly DP, et al. (2012) The discovery of new deep-sea hydrothermal vent communities in the southern ocean and implications for biogeography. PLoS Biology 10, e1001234.

Rokas A, Williams BL, King N, Carroll SB (2003) Genome-scale approaches to resolving incongruence in molecular phylogenies. Nature 425, 798-804.

Ronquist F, Teslenko M, van der Mark P, et al. (2012) MrBayes 3.2: Efficient Bayesian phylogenetic inference and model choice across a large model space. Systematic Biology 61, 539-542.

Roterman CN, Copley JT, Linse KT, Tyler PA, Rogers AD (2013) The biogeography of the yeti crabs (Kiwaidae) with notes on the phylogeny of the Chirostyloidea (Decapoda: Anomura). Proceedings of the Royal Society B: Biological Sciences 280, 20130718-20130718.

Scher HD (2006) Timing and climatic consequences of the opening of Drake Passage. Science 312, 428430.

Schwaninger HR (2008) Global mitochondrial DNA phylogeography and biogeographic history of the antitropically and longitudinally disjunct marine bryozoan Membranipora membranacea L. (Cheilostomata): Another cryptic marine sibling species complex? Molecular Phylogenetics and Evolution 49, 893-908.

Shank T, Herrera S, Bors E, et al. (2010) Hydrothermal vents and organic falls in the heart of the Coral Triangle: chemosynthetic communities discovered via relepresence in the Sangihe-Talaud region, Northern Sulawesi, Indonesia. AGU Fall Meeting Abstracts 1, 04.

Shank TM, Black MB, Halanych KM, Lutz RA, Vrijenhoek RC (1999) Miocene radiation of deep-sea hydrothermal vent shrimp (Caridea: Bresiliidae): evidence from mitochondrial cytochrome oxidase subunit I. Molecular Phylogenetics and Evolution 13, 244-254.

Shock EL, Mccollom T, Schulte MD (1995) Geochemical Constraints on Chemolithoautotrophic Reactions in Hydrothermal Systems. Origins of Life and Evolution of the Biosphere 25, 141-159.

Smith AB, Stockley B (2005) The geological history of deep-sea colonization by echinoids: roles of surface productivity and deep-water ventilation. Proceedings of the Royal Society B: Biological Sciences 272, 865-869.

Southward A (2005) Systematics and ecology of a new species of stalked barnacle (Cirripedia: Thoracica: Scalpellomorpha: Eolepadidae: Neolepadini) from the Pacific-Antarctic Ridge at $38^{\circ} \mathrm{S}$. Marine Biodiversity 35, 147-156. 
Southward AJ, Newman WA (1998) Ectosymbiosis between filamentous sulphur bacteria and a stalked barnacle (Scalpellomorpha, Neolepadinae) from the Lau Back Arc Basin, Tonga. Cahiers de Biologie Marine 39, 259-262.

Stadler T (2009) On incomplete sampling under birth-death models and connections to the samplingbased coalescent. Journal of Theoretical Biology 261, 58-66.

Stamatakis A (2006) RAxML-VI-HPC: maximum likelihood-based phylogenetic analyses with thousands of taxa and mixed models. Bioinformatics 22, 2688-2690.

Strugnell JM, Rogers AD, Prodohl PA, Collins M, Allcock AL (2008) The thermohaline expressway: the Southern Ocean as a centre of origin for deep-sea octopuses. Cladistics 24, 853-860.

Tao C, Lin J, Guo S, et al. (2011) First active hydrothermal vents on an ultraslow-spreading center: Southwest Indian Ridge. Geology 40, 47-50.

Thurber AR, Sweetman AK, Narayanaswamy BE, et al. (2014) Ecosystem function and services provided by the deep sea. Biogeosciences 11, 3941-3963.

Tsang L, Chan B, Wu T, et al. (2008) Population differentiation in the barnacle Chthamalus malayensis: postglacial colonization and recent connectivity across the Pacific and Indian Oceans. Marine Ecology Progress Series 364, 107-118.

Tsang LM, Chan BKK, Shih F-L, Chu KH, Allen Chen C (2009) Host-associated speciation in the coral barnacle Wanella milleporae (Cirripedia: Pyrgomatidae) inhabiting the Millepora coral. Molecular Ecology 18, 1463-1475.

Tufar W (1990) Modern hydrothermal activity, formation of complex massive sulfide deposits and associated vent communities in the Manus back-arc basin (bismarck Sea, Papua New Guinea). Mitteilungen der Oesterreichischen Geologischen Gesellschaft 82, 183-210.

Tunnicliffe V, Juniper SK, Sibuet M (2003) Reducing environments of the deep-sea floor. In: Ecosystems of the World: The Deep Sea (ed. Tyler PA), pp. 81-110, Amsterdam.

Tunnicliffe V, Southward AJ (2004) Growth and breeding of a primitive stalked barnacle Leucolepas longa (Cirripedia: Scalpellomorpha: Eolepadidae: Neolepadinae) inhabiting a volcanic seamount off Papua New Guinea. Journal of the Marine Biological Association of the UK 84, 121-132.

Van Dover CL (2000) The Ecology of Deep-Sea Hydrothermal Vents Princeton University Press, Princeton, N.J.

Van Dover CL (2002) Trophic relationships among invertebrates at the Kairei hydrothermal vent field (Central Indian Ridge). Marine Biology 141, 761-772.

Van Dover CL (2010) Mining seafloor massive sulphides and biodiversity: what is at risk? ICES Journal of Marine Science 68, 341-348.

Van Dover CL, Humphris SE, Fornari D, et al. (2001) Biogeography and ecological setting of Indian Ocean hydrothermal vents. Science 294, 818-823.

Van Dover CL, Smith CR, Ardron J, et al. (2012) Designating networks of chemosynthetic ecosystem reserves in the deep sea. Marine Policy 36, 378-381.

Verissimo A, McDowell JR, Graves JE (2010) Global population structure of the spiny dogfish Squalus acanthias, a temperate shark with an antitropical distribution. Molecular Ecology 19, 1651-1662.

Vrijenhoek R (2013) On the instability and evolutionary age of deep-sea chemosynthetic communities. Deep-Sea Research Part II 92, 189-200.

Wagner CE, Keller I, Wittwer S, et al. (2012) Genome-wide RAD sequence data provide unprecedented resolution of species boundaries and relationships in the Lake Victoria cichlid adaptive radiation. Molecular Ecology 22, 787-798.

Watanabe H, Tsuchida S, Fujikura K, et al. (2005) Population history associated with hydrothermal vent activity inferred from genetic structure of neoverrucid barnacles around Japan. Marine EcologyProgress Series 288, 233-240.

Whiting MF (2002) Mecoptera is paraphyletic: multiple genes and phylogeny of Mecoptera and Siphonaptera. Zoologica Scripta 31, 93-104.

Xia X, Xie Z, Salemi M, Chen L, Wang Y (2003) An index of substitution saturation and its application. Molecular Phylogenetics and Evolution 26, 1-7. 
Xia XH (2013) DAMBE5: A comprehensive software package for data analysis in molecular biology and evolution. Molecular Biology and Evolution 30, 1720-1728.

Xie WG, Lewis PO, Fan Y, Kuo L, Chen MH (2011) Improving marginal likelihood estimation for Bayesian phylogenetic model selection. Systematic Biology 60, 150-160.

Yang JS, Lu B, Chen DF, et al. (2013) When did decapods invade hydrothermal vents? Clues from the western Pacific and Indian Oceans. Molecular biology and evolution 30, 305-309.

Yoshida R, Osawa M, Hirose M, Hirose E (2011) A new fenus and two new species of Peltogastridae (Crustacea: Cirripedia: Rhizocephala) parasitizing Hermit Crabs from Okinawa Island (Ryukyu Archipelago, Japan), and their DNA-barcodes. Zoological Science 28, 853-862.

Yule GA (1925) A mathematical theory of evolution, based on the conclusions of Dr. J.C. Willis. Philosophical Transactions of the Royal Society of London B Biological Sciences 213, 21-87.

\section{SUPPORTING INFORMATION}




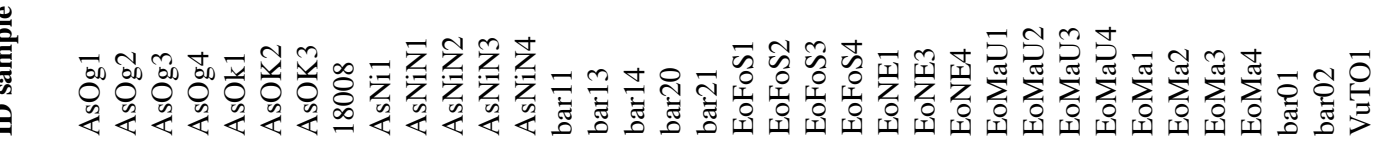

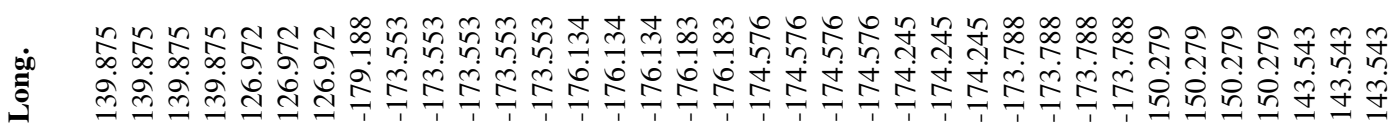

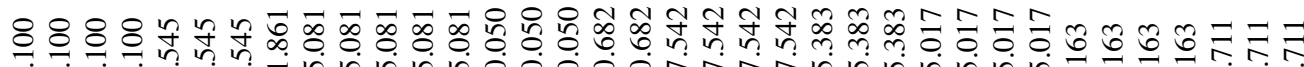

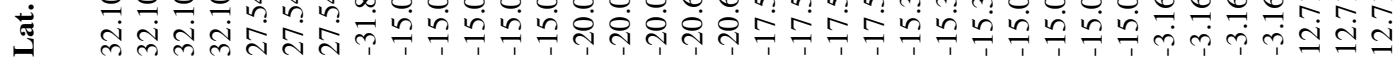

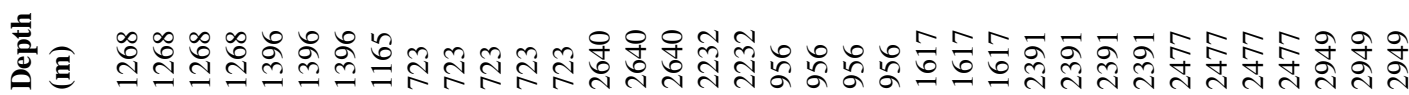

๘

峛

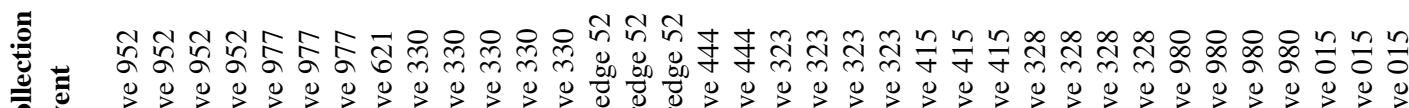

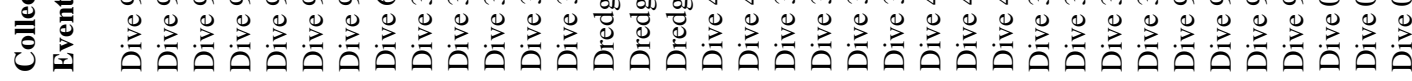

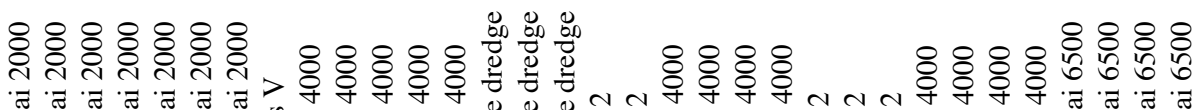

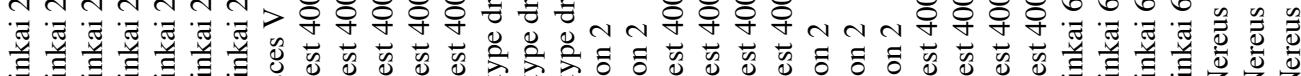

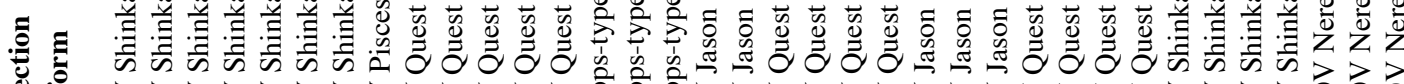

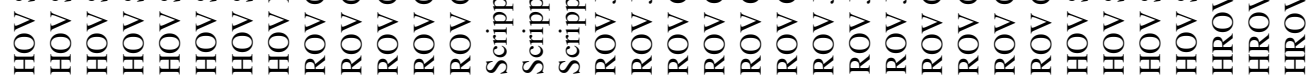

응ㅇㅇㅝ士合-ーーーーニニ

尊

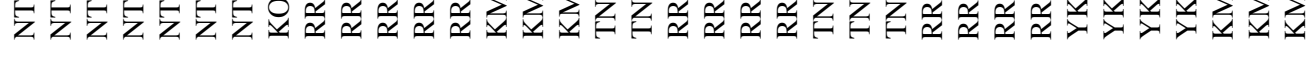

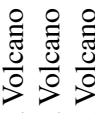

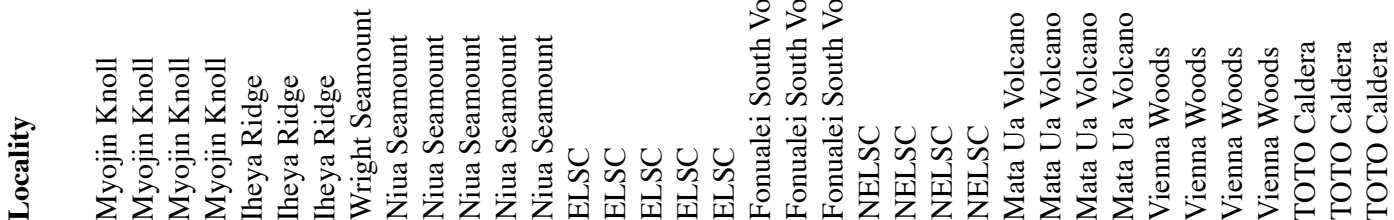

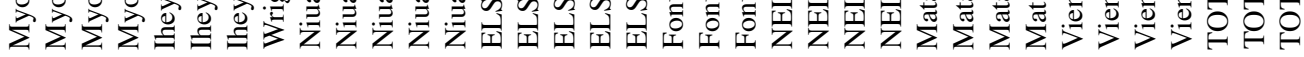

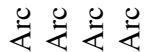

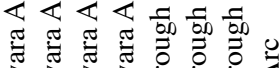

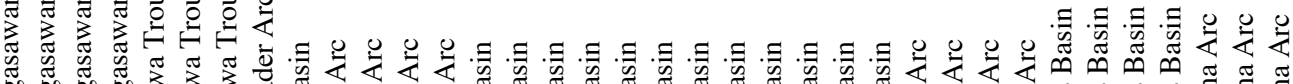

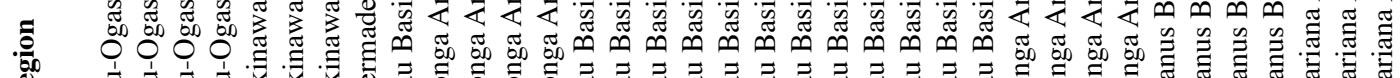

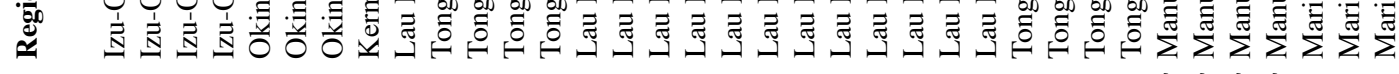

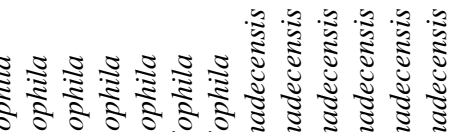

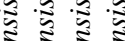

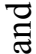

.0

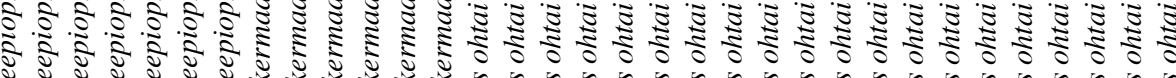

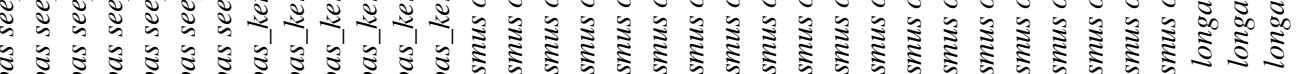

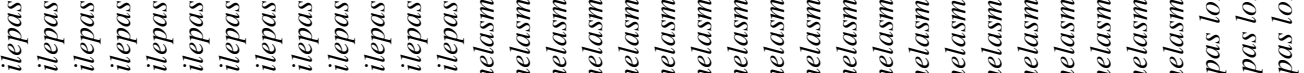

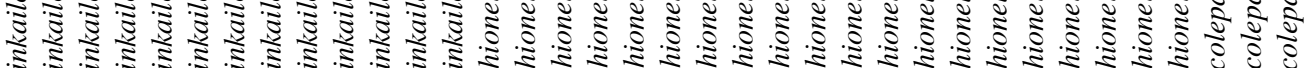

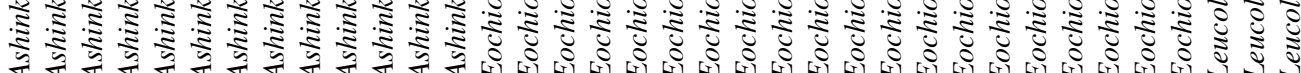




\section{$\overline{\bar{n}} \frac{N}{m} \frac{m}{m}$}

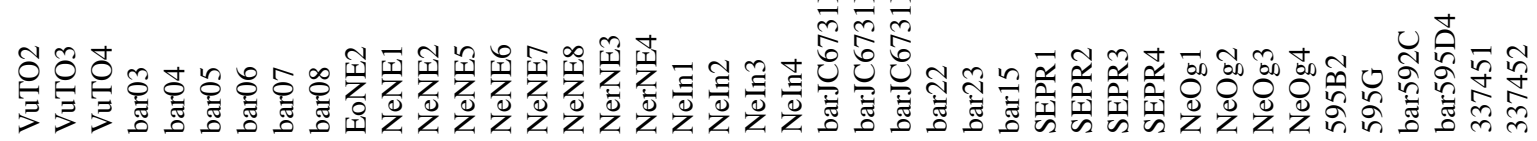

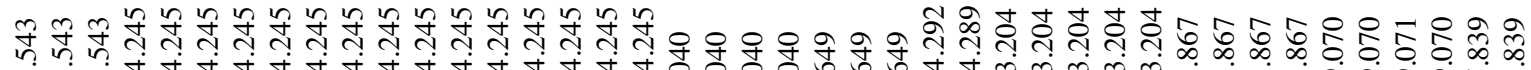

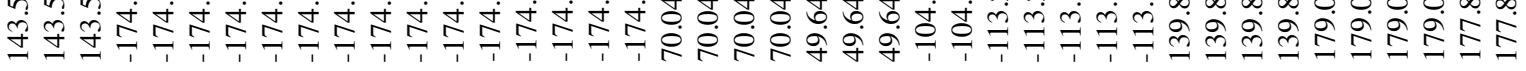

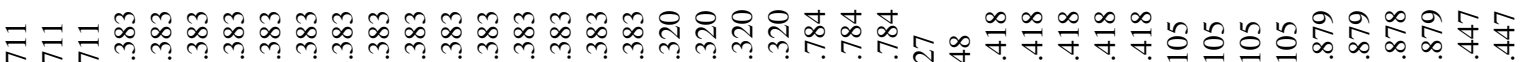

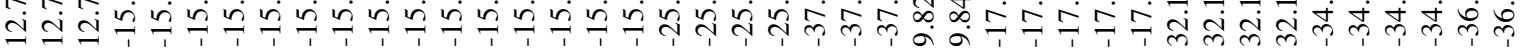

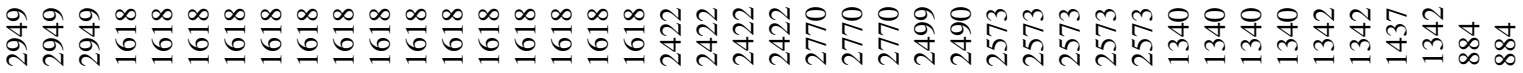
țt ஓे

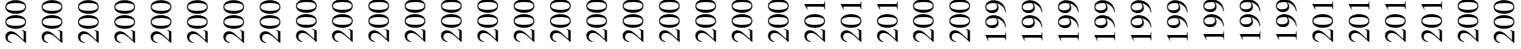

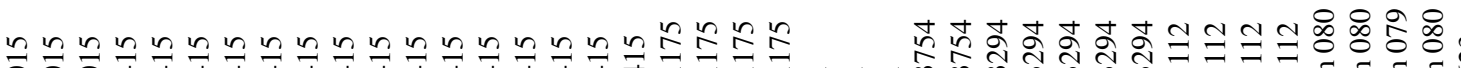

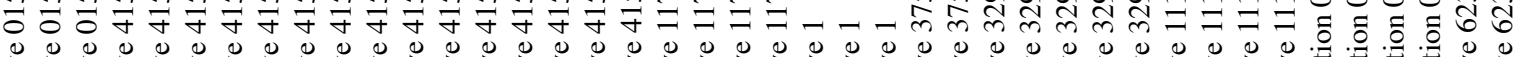

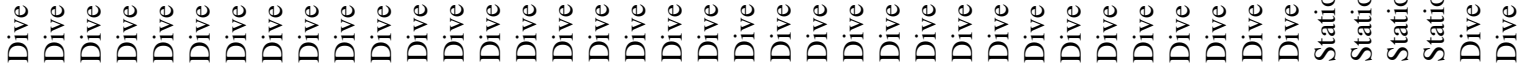

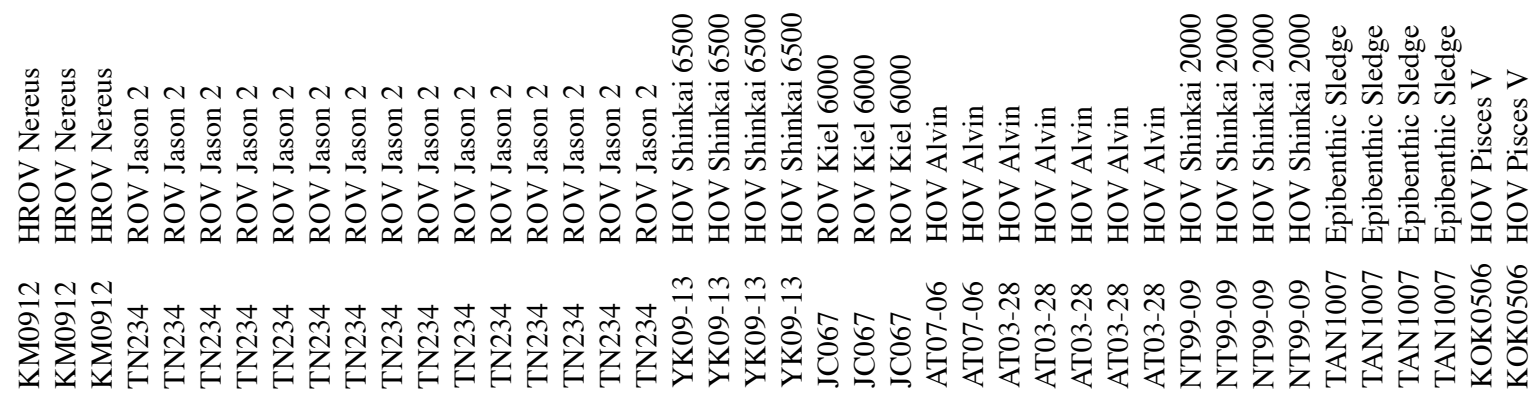

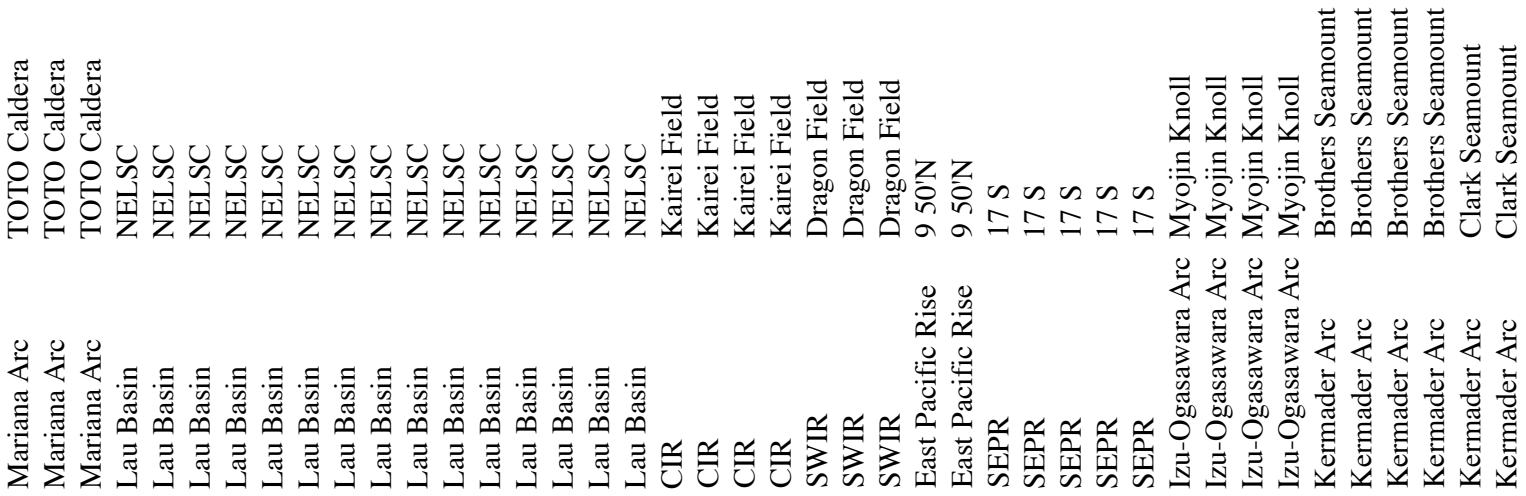

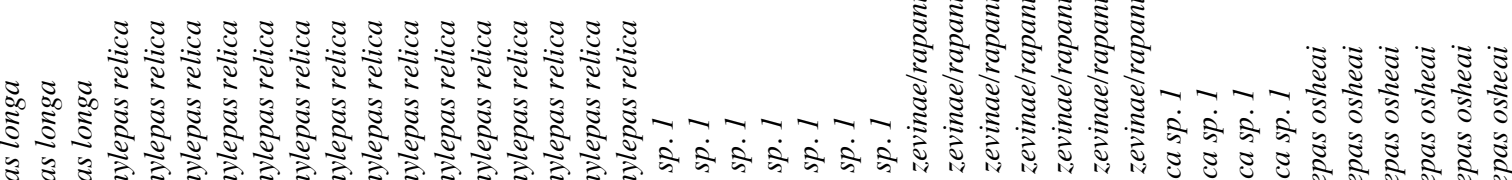

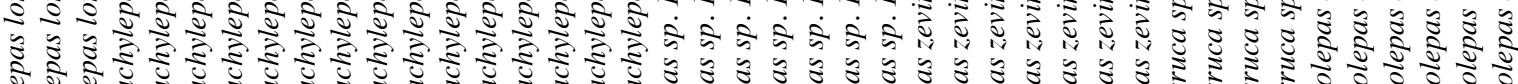

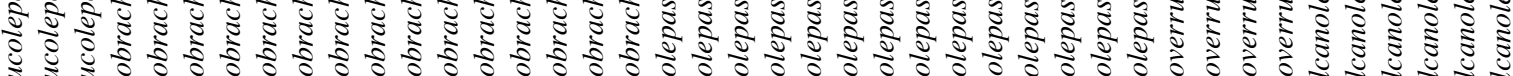

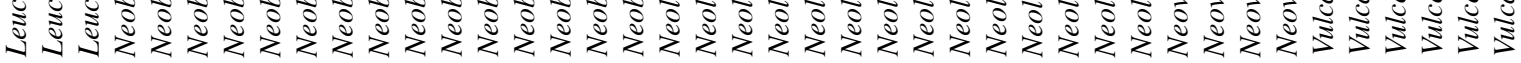




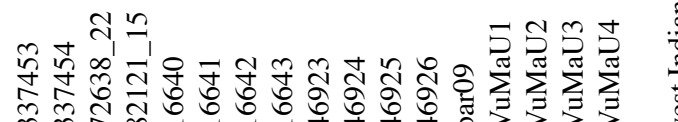

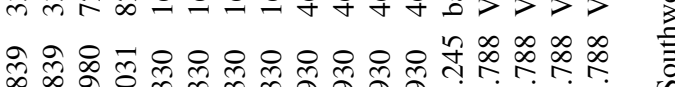

舟

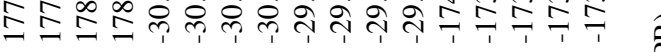

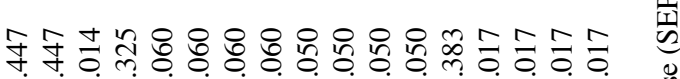

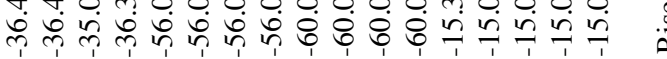

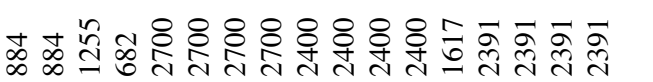

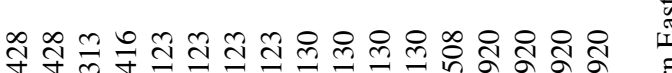

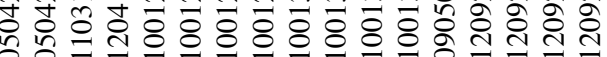

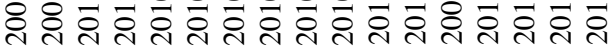

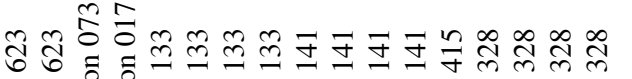

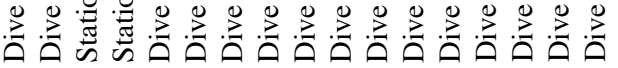

$>>\frac{\mathbb{s}_{0}}{\pi} \frac{\Delta}{\pi}$

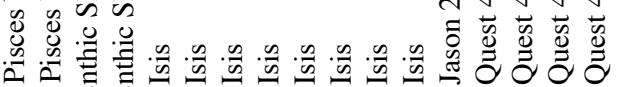

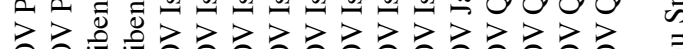

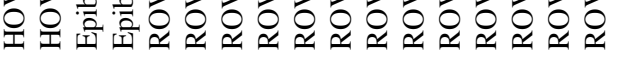

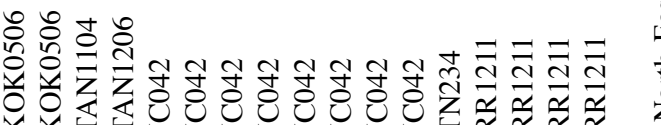

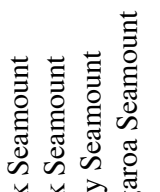

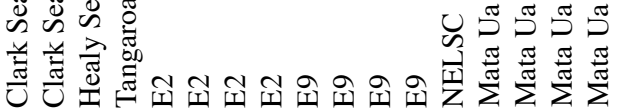

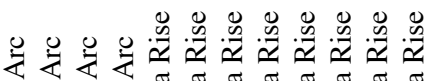

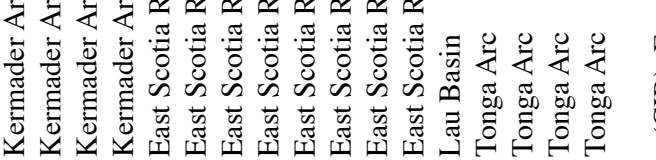

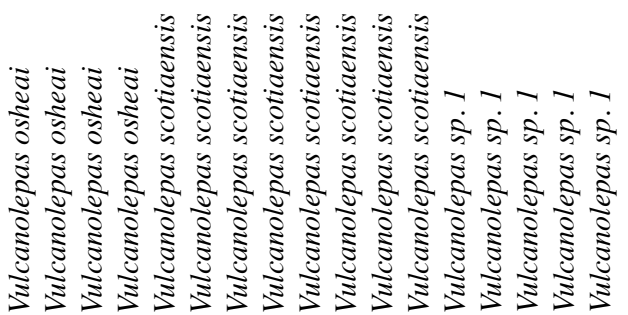

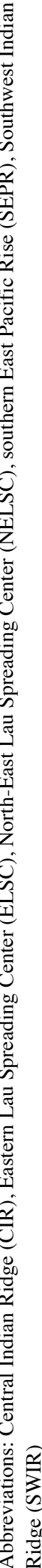


Table S2. Accession numbers for sequences from the Superorder Thoracica retrieved from GenBank

$\operatorname{cox} 1$

Taxon ID

Conchoderma hunteri

Conchoderma hunteri

Conchoderma virgatum

Lepas testudinata

Lepas testudinata

Paralepas sp HNC2013

Lepas anserifera

Heteralepas japonica

Heteralepas sp LMT2008

Heteralepas sp LMT2008

Heteralepas japonica

Heteralepas japonica

Heteralepas japonica

Paralepas sp LMT2008

Heteralepas sp LMT2008

Pseudoctomeris sulcata

Pseudoctomeris sulcata

Chthamalus sp HNC2013

Chthamalus sp HNC2013

Hexechamaesipho pilsbryi

Hexechamaesipho pilsbryi

Galkinia depressa

Megabalanus ajax

Megabalanus ajax

Megabalanus occator

Megabalanus occator

Megabalanus zebra

Megabalanus zebra

Megabalanus tintinnabulum

Megabalanus tintinnabulum

Megabalanus volcano

Megabalanus volcano

Megabalanus rosa

Megabalanus rosa

Megabalanus coccopoma

Megabalanus coccopoma

Amphibalanus zhujiangensis

Darwiniella conjugatum

Nobia conjugatum

Darwiniella conjugatum

Darwiniella conjugatum

Darwiniella conjugatum

Nobia conjugatum

Cionophorus soongi

Cionophorus soongi

Cionophorus soongi

Darwiniella angularis

Darwiniella angularis

Darwiniella angularis

Hiroa stubbingsi

Hiroa stubbingsi

Hiroa stubbingsi

Hiroa stubbingsi

\begin{tabular}{|c|c|c|}
\hline \multicolumn{3}{|l|}{$\begin{array}{l}\text { GenBank } \\
\text { Accession }\end{array}$} \\
\hline KC138462 & Amphibalanus variegatus & $\mathrm{KC} 138446$ \\
\hline KC138463 & Amphibalanus variegatus & $\mathrm{KC} 138447$ \\
\hline KC138464 & Galkinia indica & JQ946272 \\
\hline KC138477 & Galkinia indica & JQ946215 \\
\hline KC138478 & Galkinia indica & JQ946238 \\
\hline KC138502 & Galkinia indica & JQ946273 \\
\hline KC138474 & Galkinia equus & JQ946276 \\
\hline FJ694788 & Galkinia equus & JQ946251 \\
\hline EU884170 & Galkinia tabulatus & JQ946255 \\
\hline EU884172 & Galkinia altiapiculus & JQ946270 \\
\hline EU884154 & Galkinia altiapiculus & JQ946287 \\
\hline EU884153 & Galkinia altiapiculus & JQ946271 \\
\hline EU884169 & Galkinia altiapiculus & JQ946286 \\
\hline EU884174 & Galkinia depressa & JQ946289 \\
\hline EU884171 & Galkinia depressa & JQ946285 \\
\hline KC138503 & Galkinia depressa & JQ946288 \\
\hline KC138504 & Armatobalanus allium & $\mathrm{KC} 138450$ \\
\hline KC138465 & Cantellius hoegi & FJ379314 \\
\hline KC138466 & Cantellius hoegi & KC138453 \\
\hline KC138470 & Cantellius hoegi & FJ379315 \\
\hline KC138471 & Cantellius hoegi & KC138454 \\
\hline JQ946262 & Cantellius sextus & FJ379311 \\
\hline KC138480 & Cantellius sextus & $\mathrm{KC} 138456$ \\
\hline KC138479 & Cantellius sextus & FJ379312 \\
\hline KC138483 & Cantellius sextus & KC138457 \\
\hline KC138484 & Nobia grandis & FJ379318 \\
\hline KC138491 & Nobia grandis & KC138496 \\
\hline KC138492 & Nobia grandis & KC138497 \\
\hline KC138487 & Balanus trigonus & $\mathrm{KC} 138451$ \\
\hline KC138488 & Balanus trigonus & $\mathrm{KC} 138452$ \\
\hline KC138489 & Wanella milleporae & EF565204 \\
\hline KC138490 & Wanella milleporae & $\mathrm{KC} 138514$ \\
\hline $\mathrm{KC} 138485$ & Wanella milleporae & $\mathrm{KC} 138515$ \\
\hline KC138486 & Amphibalanus amphitrite & KC138445 \\
\hline KC138481 & Fistulobalanus albicostatus & KC138468 \\
\hline KC138482 & Fistulobalanus albicostatus & $\mathrm{KC} 138469$ \\
\hline KC138448 & Membranobalanus longirostrum & KC138493 \\
\hline JQ988964 & Cantellius pallidus & FJ379317 \\
\hline EF569557 & Wanella milleporae & EF565206 \\
\hline JQ988945 & Tetraclitella karandei & $\mathrm{KC} 138510$ \\
\hline JQ988946 & Tetraclitella karandei & KC138511 \\
\hline JQ989033 & Tetraclitella chinensis & KC138506 \\
\hline EF569556 & Yamaguchiella sp HNC2013 & $\mathrm{KC} 138512$ \\
\hline JQ946277 & Yamaguchiella sp HNC2013 & KC138513 \\
\hline JQ946278 & Newmanella sp HNC2013 & KC138494 \\
\hline JQ946279 & Newmanella sp HNC2013 & KC138495 \\
\hline JQ988974 & Chelonibia patula & JF823664 \\
\hline JQ988978 & Chelonibia patula & JF823661 \\
\hline JQ988980 & Chelonibia testudinaria & KC138460 \\
\hline JQ946244 & Chelonibia patula & JF823663 \\
\hline JQ946246 & Chelonibia patula & JF823662 \\
\hline JQ946237 & Chelonibia testudinaria & KC138461 \\
\hline JQ946245 & Chelonibia testudinaria & JF823739 \\
\hline
\end{tabular}


Chelonibia testudinaria

Tetraclita kuroshioensis

Tetraclitella chinensis

Darwiniella angularis

Capitulum mitella

Capitulum mitella

Ibla cumingi

Ibla cumingi

Galkinia equus

Tetraclitella divisa

Tetraclitella divisa

Chthamalus sp 1 JDZ2005

Tesseropora wireni pacifica

Amphibalanus improvisus

Trevathana mizrachae

Trevathana mizrachae

Trevathana mizrachae

Trevathana mizrachae

Trevathana margaretae

Trevathana margaretae

Trevathana margaretae

Trevathana dentata

Trevathana dentata

Trevathana dentata

Trevathana jensi

Trevathana jensi

Trevathana jensi

Trevathana jensi

Trevathana sarae

Trevathana sarae

Savignium crenatum

Savignium crenatum

Savignium crenatum

Neotrevathana elongatum

Trevathana sarae

Trevathana sarae

Savignium crenatum

Trevathana margaretae

Semibalanus balanoides

Tetraclita rubescens

Lepas anatifera

Lepas anatifera

Lepas anatifera

Lepas pectinata

Lepas pectinata

Lepas pectinata

Lepas anserifera

Lepas anserifera

Lepas australis

Lepas australis

Lepas australis

Lepas australis

Lepas anatifera

Tetrachthamalus oblitteratus

Chamaesipho sp BOLDAAW6872

Tetraclita singaporensis

Tetraclita singaporensis

Tetraclita singaporensis
JF823740

$\mathrm{KC} 138505$

$\mathrm{KC} 138507$

JQ988981

KC138458

$\mathrm{KC} 138459$

$\mathrm{KC} 138472$

$\mathrm{KC} 138473$

JQ946226

KC138508

$\mathrm{KC} 138509$

AY823025

AY823032

FJ845840

FJ620805

FJ620802

FJ620803

FJ620804

FJ620810

FJ620811

FJ620812

FJ620820

FJ620821

FJ620822

FJ620823

FJ620824

FJ620825

FJ620826

FJ620798

FJ620800

FJ620792

FJ620789

FJ620791

FJ620831

FJ620830

FJ620799

FJ620790

FJ620813

FJ845815

GU381928

GU993589

GU993590

GU993591

GU993645

GU993644

GU993650

GU993630

GU993629

GU993638

GU993639

GU993641

GU993640

GU993588

AY430813

DQ889089

EF035166

EF035164

EF035165
Tetraclita formosana

Tetraclita japonica

Tetraclita japonica

Tetraclita formosana

Tetraclita japonica

Tetraclita formosana

Tetraclita japonica

Tetraclita formosana

Tetraclita singaporensis

Tesseropora rosea

Tesseropora rosea

Tesseropora rosea

Tesseropora rosea

Tetraclita rubescens

Catomerus polymerus

Catomerus polymerus

Catomerus polymerus

Catomerus polymerus

Tetraclita rubescens

Chthamalus of challengeri JDZ2005

Tetrachthamalus sinensis

Chinochthamalus scutelliformis

Chthamalus fragilis

Chthamalus bisinuatus

Conopea sp A DCS2011

Tetraclita rubescens

Chthamalus moro

Tetraclitella purpurascens

Tetraclitella purpurascens

Tetraclitella purpurascens

Tetraclitella purpurascens

Hexechamaesipho pilsbryi

Pollicipes pollicipes

Pollicipes pollicipes

Pseudoctomeris sulcata

Chamaesipho tasmanica

Capitulum mitella

Chthamalus angustitergum

Chthamalus angustitergum

Chthamalus angustitergum

Chthamalus angustitergum

Chthamalus bisinuatus

Chthamalus bisinuatus

Chthamalus bisinuatus

Microeuraphia depressa

Microeuraphia depressa

Microeuraphia depressa

Chthamalus montagui

Chthamalus montagui

Chthamalus montagui

Chthamalus montagui

Chthamalus stellatus

Chthamalus stellatus

Microeuraphia rhizophorae

Microeuraphia rhizophorae

Microeuraphia rhizophorae

Euraphia eastropacensis

Euraphia eastropacensis
DQ647761

DQ647704

DQ647707

DQ647764

DQ647706

DQ647763

DQ647705

DQ647762

EF035167

FJ516076

FJ516077

FJ516074

FJ516075

GU381926

FJ516172

FJ516173

FJ516175

FJ516174

GU381927

AY823019

JQ755178

JQ755182

JQ755179

FJ845849

JQ966291

GU381925

EU304448

FJ516126

FJ516110

FJ516127

FJ516125

KC896275

EF462950

EF462951

JX083865

JX083867

JX502998

FJ845832

FJ845836

FJ845839

FJ845833

FJ845850

FJ845845

FJ845846

HQ224880

EF095159

EF095160

FJ858061

FJ858066

FJ858062

FJ858060

EU699247

EU699241

FJ845864

FJ845865

FJ845866

FJ845851

FJ845852 
Euraphia eastropacensis

Chthamalus neglectus

Chthamalus neglectus

Chthamalus neglectus

Chthamalus neglectus

Chthamalus malayensis

Chthamalus malayensis

Chthamalus malayensis

Chthamalus malayensis

Chthamalus dentatus

Chthamalus dentatus

Chthamalus dentatus

Chthamalus dentatus

Chthamalus antennatus

Euraphia eastropacensis

Chthamalus cortezianus

Chthamalus cortezianus

Chthamalus cortezianus

Microeuraphia sp 2 MPL2012

Euraphia sp 2 MPL2012

Chthamalus challengeri

Chthamalus challengeri

Chthamalus challengeri

Chthamalus challengeri

Hexechamaesipho pilsbryi

Caudoeuraphia caudata

Megatrema anglicum

Nesochthamalus intertextus

Megabalanus rosa

Megabalanus rosa

Balanus glandula

Balanus glandula

Balanus glandula

Balanus glandula

Darwiniella sp HNC2013

Conopea galeata

Conopea galeata

Chamaesipho columna

Semibalanus cariosus

Semibalanus cariosus

Semibalanus cariosus

Semibalanus cariosus

Semibalanus balanoides

Semibalanus balanoides

Semibalanus balanoides

Austrobalanus imperator

Tetraclita kuroshioensis

Amphibalanus improvisus

Amphibalanus improvisus

Amphibalanus improvisus

Verruca stroemia

Catophragmus imbricatus

Microeuraphia rhizophorae

Chthamalus panamensis

Chthamalus panamensis

Chthamalus panamensis

Chthamalus panamensis

Chthamalus hedgecocki
FJ845858

FJ858077

FJ858079

FJ858080

FJ858078

FJ845828

FJ845830

FJ845831

FJ845829

FJ858084

FJ858086

FJ858087

FJ858088

JX083870

FJ845857

AF234810

AF234811

AF234812

JX083873

JX083872

FJ858069

FJ858073

FJ858074

FJ858075

JX083868

JX083871

FJ713101

JX083869

JX503004

JX503005

EF694592

EF694596

EF694594

EF694595

KC138467

JQ966287

JQ966288

JX083866

GQ902335

GQ902333

GQ902332

GQ902334

FJ845819

FJ845818

GQ328964

EU423232

JX186409

FJ845841

FJ845844

FJ845842

JX083863

JX083864

FJ845863

FJ857976

FJ857974

FJ857960

FJ857966

FJ857986
Chthamalus hedgecocki

FJ857987

Chthamalus hedgecocki

Chthamalus southwardorum complex sp B

Chthamalus southwardorum complex $s p B$

Chthamalus southwardorum complex sp $B$

Chthamalus southwardorum complex sp $B$

Chthamalus fragilis

Chthamalus fragilis

Chthamalus anisopoma

Chthamalus proteus

Chthamalus proteus

Chthamalus proteus

Chthamalus proteus

Chthamalus mexicanus

Chthamalus mexicanus

Chthamalus mexicanus

Capitulum mitella

Chthamalus hedgecocki

Fistulobalanus albicostatus

Chthamalus anisopoma

Chthamalus anisopoma

Chthamalus anisopoma

Microeuraphia depressa

Chthamalus stellatus

Chthamalus stellatus

Microeuraphia withersi

Chamaesipho brunnea

Jehlius cirratus

Jehlius cirratus

Jehlius cirratus

Jehlius cirratus

Octomeris angulosa

Notochthamalus scabrosus

Notochthamalus scabrosus

Notochthamalus scabrosus

Pollicipes polymerus

Pollicipes polymerus

Pollicipes polymerus

Pollicipes polymerus

Pollicipes caboverdensis

Pollicipes caboverdensis

Pollicipes caboverdensis

Pollicipes caboverdensis

Pollicipes pollicipes

Pollicipes pollicipes

Pollicipes pollicipes

Pollicipes pollicipes

Galkinia adamanteus

Octomeris brunnea

Galkinia decima

Cantellius septimus

Tetraclita pacifica

Tetraclita pacifica

Tetraclita pacifica

Tetraclita pacifica

Tetraclita squamosa

Tetraclita squamosa

Tetraclita squamosa
FJ857990

FJ857992

FJ857997

FJ857999

FJ857993

AF234807

AF234813

AF234816

FJ858023

FJ858025

FJ858039

FJ858027

AF234804

AF234805

AF234803

JX502999

FJ857989

JX503003

AF234818

AF234817

AF234819

HQ224881

EU699240

EU699243

AY430814

AY430811

GU126095

GU126107

GU126087

GU126116

AY428049

FJ845821

FJ845822

GU125776

GU442485

GU442491

GU442492

GU442494

HM563665

HM563666

HM563667

HM563668

HM563675

HM563676

HM563678

HM563677

JX983109

AY430812

JQ946213

KC138455

DQ363694

DQ363692

DQ363693

DQ363695

DQ363704

DQ363705

DQ363703 
Tetraclita squamosa

Conopea calceola

Conopea calceola

Conopea sp B DCS2011

Conopea sp B DCS2011

Conopea sp B DCS2011

Conopea sp B DCS2011

Conopea calceola

Conopea sp A DCS2011

Conopea sp A DCS2011

Conopea sp A DCS2011

Conopea cf galeata USA DCS2011

Conopea cf galeata Panama DCS2011

Conopea cf galeata Brazil DCS2011

Conopea cf galeata Galapagos DCS2011

Chthamalus moro

Chthamalus moro

Tetraclita stalactifera

Chelonibia caretta

Chelonibia caretta

Chelonibia caretta

Chthamalus dalli

Chthamalus dalli

Chthamalus dalli

Chthamalus dalli

Chthamalus fissus

Chthamalus fissus

Chthamalus fissus

Chthamalus fissus

Chthamalus sp kino

Chthamalus sp kino

Chthamalus sp kino

Chthamalus sp kino

Tetraclita ehsani

Tetraclita ehsani

Tetraclita ehsani

Tetraclita ehsani

Tetraclita serrata

Tetraclita serrata

Tetraclita serrata

Tetraclita serrata

Tetraclita sp n LMT2012

Tetraclita sp n LMT2012

Tetraclita sp n LMT2012

Tetraclita sp n LMT2012

Tetraclita reni

Tetraclita reni

Tetraclita achituvi

Tetraclita achituvi

Tetraclita achituvi

Tetraclita achituvi

Lepas anserifera

Lepas pectinata

Verruca laevigata

Armatobalanus allium

Rostratoverruca krugeri

Verruca sp CJS2008

Brochiverruca sp KT0203a
DQ363706

HQ290142

HQ290143

HQ290138

HQ290141

HQ290139

HQ290140

HQ290134

HQ290135

HQ290136

HQ290137

HQ290146

HQ290130

HQ290133

HQ290144

HM135959

HM135960

JN589833

JN589810

JN589812

JN589811

AY795282

AY795283

AY795285

AY795284

DQ538424

DQ538422

DQ538421

DQ538423

DQ538449

DQ538448

DQ538447

DQ538446

JX186296

JX186295

JX186297

JX186298

JX186199

JX186201

JX186200

JX186202

JX186368

JX186366

JX186369

JX186367

JX186294

JX186365

JX186290

JX186291

JX186289

JX186288

$\mathrm{KC} 138475$

$\mathrm{KC} 138476$

JX083862

KC138449

AB195609

EU439973

AB195608
Altiverruca sp NT0207\&08

Metaverruca recta

Octolasmis angulata

Octolasmis cor

Octolasmis cor

Octolasmis orthogonia

Octolasmis warwickii

Neoverruca brachylepadoformis

Neoverruca sp Ok8

Neoverruca sp Ok14

Neoverruca sp Ok6

Neoverruca sp Ok9

Neoverruca sp Ok1

Neoverruca sp Ok5

Neoverruca sp Ok15

Neoverruca sp Ok7

Neoverruca sp Ok11

Neoverruca sp Ok13

Neoverruca sp Ok10

Neoverruca sp Og5

Neoverruca sp Og1

Neoverruca sp Og4

Neoverruca sp Og3

Neoverruca sp Og6

Neoverruca sp $\mathrm{Og} 7$

Neoverruca sp Og9

Neoverruca sp Og8

Neoverruca sp Ok4

Neoverruca sp Ok12

Neoverruca sp Ok3

Neoverruca sp Og2

Neoverruca sp Ok2

Chthamalus sp 2 JDZ2005

Chthamalus sp 2 JDZ2005

Chthamalus sp 2 JDZ2005

Chthamalus sp 2 JDZ2005

Pollicipes sp JQ2009

Pollicipes sp JQ2009

Pollicipes sp JQ2009

Pollicipes sp JQ2009

Pollicipes elegans

Pollicipes elegans

Pollicipes elegans

Pollicipes elegans

Notochthamalus scabrosus

Calantica spinosa

Notochthamalus scabrosus

Calantica spinosa
AB 195607

JX083861

KC138498

KC138499

KC138500

EU884173

$\mathrm{KC} 138501$

AB195606

AB195598

AB195604

AB195596

AB195599

AB195591

AB195595

AB195605

AB195597

AB195601

AB195603

AB195600

AB195586

AB195582

AB195585

AB195584

AB195587

AB195588

AB195590

AB195589

AB195594

AB195602

AB195593

AB195583

AB195592

AY823028

AY 823030

AY823029

AY823031

GQ472625

GQ472627

GQ472628

GQ472626

GQ472619

GQ472614

GQ472615

GQ472618

FJ845824

AY428047

FJ84582

AY428047 
Taxon ID

Altiverruca sp KACb00436

Metaverruca recta

Verruca laevigata

Verruca stroemia

Rostratoverruca sp KACb00435

Rostratoverruca krugeri

Chelonibia caretta

Chelonibia manati

Chelonibia patula

Megabalanus occator

Megabalanus tintinnabulum

Megabalanus californicus

Megabalanus californicus

Austromegabalanus psittacus

Megabalanus spinosus

Balanus perforatus

Menesiniella aquila

Stomatolepas praegustator

Stomatolepas sp RH2012

Stomatolepas transversa

Tubicinella cheloniae

Platylepas decorata

Cylindrolepas sinica

Xenobalanus globicipitis

Coronula diadema

Cryptolepas rhachianecti

Tetraclita squamosa

Tetraclita japonica

Platylepas hexastylos

Cylindrolepas darwiniana

Austrobalanus imperator

Catomerus polymerus

Catophragmus imbricatus

Pseudoctomeris sulcata

Elminius modestus

Elminius kingii

Notochthamalus scabrosus

Octomeris angulosa

Chamaesipho brunnea

Chamaesipho tasmanica

Chamaesipho sp MPL2012

Nesochthamalus intertextus

Chamaesipho columna

Chthamalus stellatus

Chthamalus montagui

Chthamalus challengeri

Jehlius cirratus

Microeuraphia withersi

Chthamalus bisinuatus

Tetrachthamalus oblitteratus

Caudoeuraphia caudata

Chthamalus malayensis

Chthamalus dentatus

Chthamalus anisopoma

Chthamalus antennatus
GenBank

Accession

EU082300

EU082297

EU082296

AY520615

EU082298

EU082299

AB723915

AB723917

EU082295

AB723916

AY520597

AY520598

AY859588

AY520600

AY520599

AY520595

AY520596

AB723919

AB723921

AB723920

AB723922

AB723923

AB723926

AB723927

AB723928

AB723929

AY520605

AY520606

AB723924

AB723925

AB723930

AY520614

JX083911

JX083912

AY520601

AY520602

AY520612

JX083916

JX083915

AY520613

JX083914

JX083919

JX083913

AY520607

AY520608

AY520609

AY520611

JX083928

AY520610

JX083925

JX083926

JX083922

JX083923

JX083924

JX083920 $\begin{array}{lr}\text { Microeuraphia depressa } & \text { JX083927 }\end{array}$

Octomeris brunnea JX083917

Hexechamaesipho pilsbryi JX083918

Pachylasma japonicum AB723931

Stephanolepas muricata AB723918

Tetraclitella divisa AY520603

Tetraclitella purpurascens $\quad$ AY520604

Semibalanus balanoides $\quad$ AY520592

Semibalanus balanoides $\quad$ EU370440

Semibalanus cariosus AY520593

Microeuraphia rhizophorae JX083929

Lithotrya valentiana EU082301

Lithotrya sp KACb00393 EU082302

Balanus crenatus

Balanus glandula

Balanus balanus

Pollicipes pollicipes

Pollicipes pollicipes

Lepas sp Lep 1

Pollicipes polymerus

Capitulum mitella

Calantica spinosa

Calantica sp KACb00087

Smilium peronii

Litoscalpellum discoveryi

Trianguloscalpellum regium

Litoscalpellum regina

Scalpellum scalpellum

Arcoscalpellum sp CJS2008

Arcoscalpellum sp CJS2008

Arcoscalpellum africanum

Arcoscalpellum africanum

Arcoscalpellum africanum

Arcoscalpellum africanum

Litoscalpellum sp CJS2008

Litoscalpellum sp CJS2008

Scalpellum sp CJS2008

Ornatoscalpellum stroemii

Arcoscalpellum beuveti

Leucolepas longa

Vulcanolepas sp KACb00419

Vulcanolepas osheai

Neolepas rapanuii

Neolepas zevinae

Ashinkailepas seepiophila

Neoverruca sp KACb00361

Neoverruca sp KACb00389

AY520590

AY520591

AY520594

AY520616

EU370441

EU914256

AY520617

AY520618

EU082303

EU082304

EU082305

EU489824

EU082308

AY520619

EU082307

EU489828

EU489829

EU489831

EU489834

EU489835

EU489833

EU489837

EU489839

EU489830

EU082306

EU489827

EU082311

EU082312

EU082313

EU082309

EU082310

EU082314

EU082315

EU082316

EU082317

AY520621

EU082332

AY520620

EU082329

EU082330

EU082326

EU082327 
Octolasmis warwickii

Conchoderma auritum

Conchoderma virgatum

Lepas pectinata

Lepas anserifera

Lepas australis

Lepas testudinata

Paralepas dannevigi

Oxynaspis celata

Heteralepadomorpha sp KACb00398

\section{H3}

\section{Taxon ID}

Vulcanolepas sp KACb00419

Vulcanolepas osheai

Neolepas zevinae

Leucolepas longa

Neolepas rapanuii

Ashinkailepas seepiophila

Vulcanolepas sp East Scotia Ridge

Neoverruca sp KACb00389

Neoverruca sp KACb00361

Neoverruca brachylepadoformis

Lithotrya sp KACb00393

Lithotrya valentiana

Tesseropora rosea

Tetraclita achituvi

Tetraclita rufotincta

Tetraclita sp n LMT2012

Tetraclita sp n LMT2012

Tetraclita sp n LMT2012

Tetraclita sp n LMT2012

Tetraclita ehsani

Tetraclita kuroshioensis

Tetraclita serrata

Tetraclita serrata

Tetraclita serrata

Trianguloscalpellum regium

Chelonibia patula

Metaverruca recta

Verruca laevigata

Conopea calceola

Conopea calceola

Conopea calceola

Conopea fidelis

Conopea sp B DCS2011

Conopea fidelis

Conopea sp B DCS2011

Conopea sp B DCS2011

Conopea fidelis

Conopea sp B DCS2011

Conopea calceola

Conopea calceola

Conopea sp A DCS2011

Conopea saotomensis

Conopea saotomensis

Conopea saotomensis
EU082328

EU082320

EU082321

EU082322

EU082323

EU082324

EU082325

EU082318

EU082331

EU082319

GenBank

Accession

EU082352

EU082353

EU082350

EU082351

EU082349

EU082354

JN628252

EU082356

EU082355

EU082357

EU082343

EU082342

JX186507

JX186499

JX186501

JX186504

JX186502

JX186505

JX186503

JX186500

JX186506

JX186497

JX186496

JX186498

EU082348

EU082337

EU082339

EU082338

HQ290156

HQ290155

KC349910

KC349914

HQ290150

KC349912

HQ290158

HQ290157

KC349905

HQ290151

HQ290152

HQ290149

HQ290160

KC349911

KC349906

KC349913
Conopea saotomensis

Conopea saotomensis

Conopea sp A DCS2011

Conopea saotomensis

Conopea saotomensis

Rostratoverruca krugeri

Rostratoverruca sp KACb00435

Megalasma striatum

Octolasmis sp KACb00064

Lepas anserifera

Lepas australis

Lepas testudinata

Lepas pectinata

Octolasmis cor

Oxynaspis celata

Heteralepadomorpha sp KACb00398

Octolasmis warwickii

Scalpellum scalpellum

Calantica sp KACb00087

Smilium peronii

Calantica spinosa

Pyrgopsella sp SMB2011

Savignium crenatum

Conchoderma auritum

Conchoderma virgatum

Conopea cf galeata Galapagos DCS2011

Conopea cf galeata Galapagos DCS2011

Conopea cf galeata USA DCS2011

Conopea galeata

Conopea galeata

Conopea galeata

Conopea galeata

Paralepas dannevigi

Pollicipes elegans

Pollicipes sp RJVS2010

Poecilasma kaempferi

Ibla cumingi
KC349909

KC349904

HQ290159

KC349907

KC349908

EU082341

EU082340

EU082370

EU082367

EU082363

EU082364

EU082365

EU082362

EU082366

EU082371

EU082359

EU082368

EU082347

EU082345

EU082346

EU082344

JN800715

JN800716

EU082360

EU082361

HQ290162

HQ290161

HQ290147

JQ966286

JQ966283

JQ966284

JQ966285

EU082358

HM142348

HM142349

EU082369

EU082372 


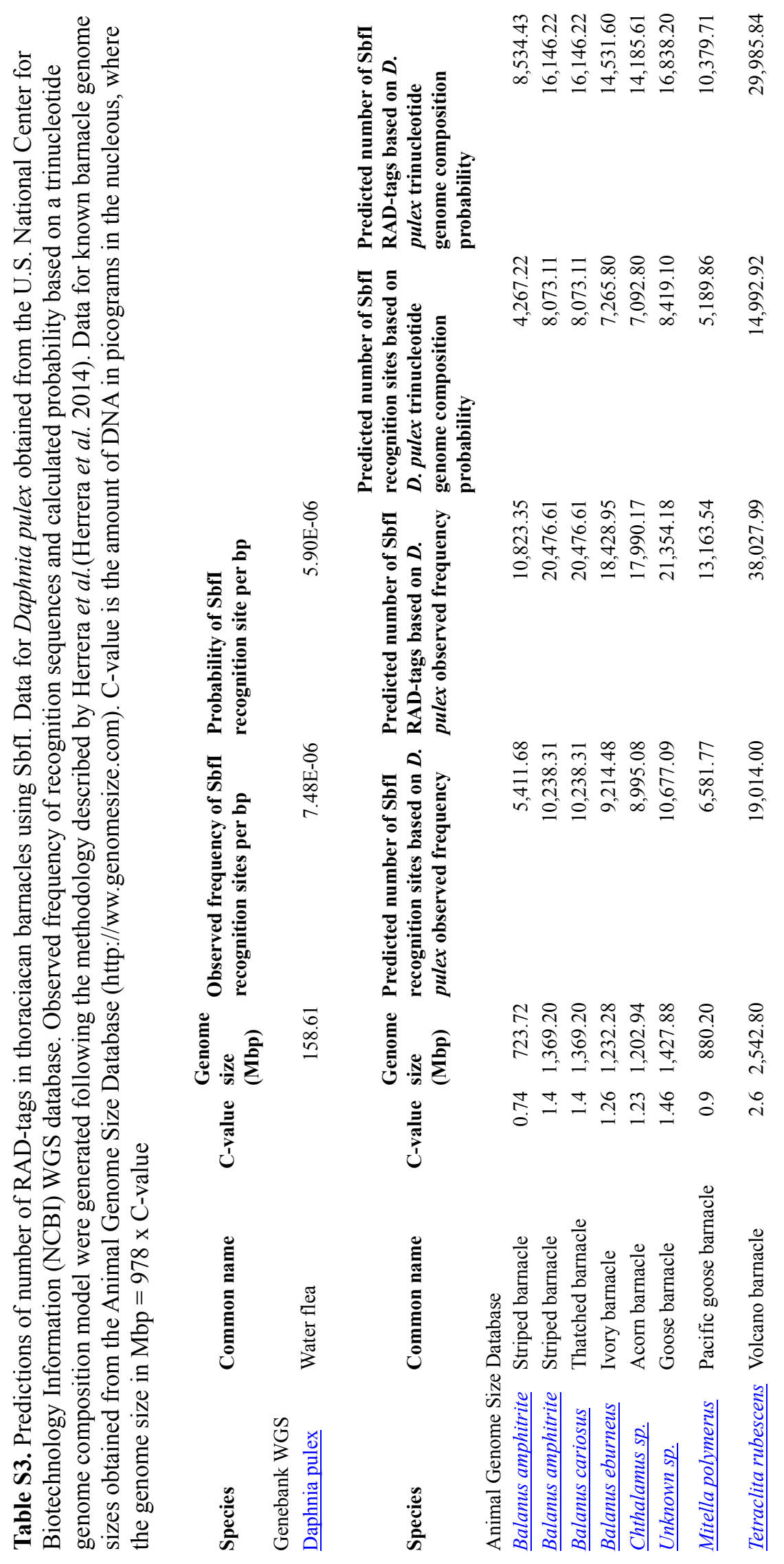


Table S4. Nucleotide substitution models for each Sanger-based genetic marker, as selected by the BIC criterion in jModeltest.

Dataset

Thoracica

Clade A

coxI_codon12
GTR $+\mathrm{I}+\mathrm{G}$
$\mathrm{HKY}+\mathrm{I}$

H3_codon12

H3 codon 3

$\mathrm{HKY}+\mathrm{I}+\mathrm{G}$

$\mathrm{HKY}+\mathrm{I}$
$28 S$

$\mathrm{GTR}+\mathrm{I}+\mathrm{G}$

$\mathrm{HKY}+\mathrm{G}$

Table S5. Results from Xia saturation test for each Sanger-based genetic marker.

$\begin{array}{lcccc}\text { Dataset } & \text { coxI_codon12 } & \text { H3_codon12 } & \text { H3_codon3 } & \text { 28S } \\ \text { Thoracica } & \text { Little saturation } & \text { Substantial saturation } & \text { Little saturation } & \text { Little saturation } \\ \text { C1 } & \text { Little saturation } & \text { Substantial saturation } & \text { Little saturation } & \text { Little saturation }\end{array}$




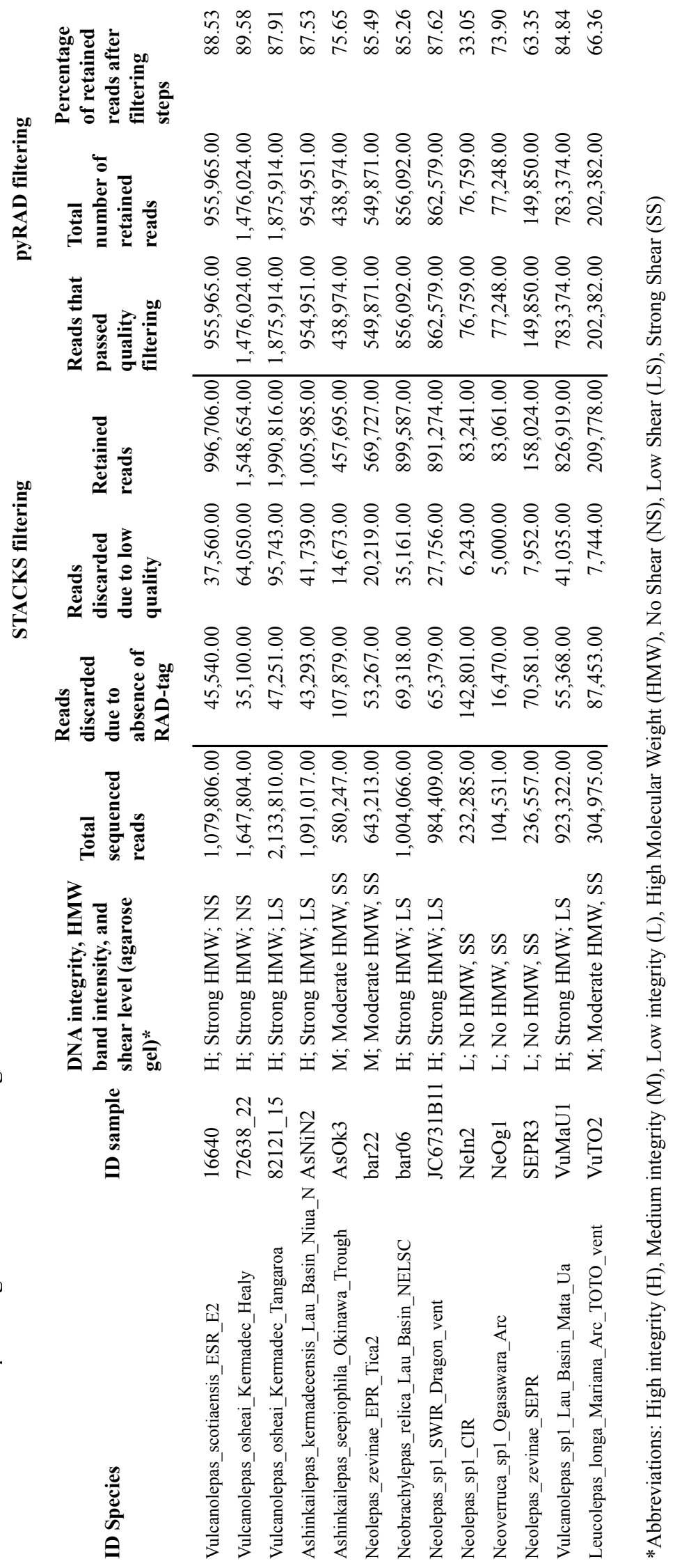


Table S7. RAD clustering statistics.

\begin{tabular}{|c|c|c|c|c|c|c|}
\hline \multirow[b]{2}{*}{ ID sample } & \multicolumn{6}{|c|}{ pyRAD clustering (c80) } \\
\hline & $\begin{array}{l}\text { Total number } \\
\text { of clusters }\end{array}$ & $\begin{array}{l}\text { Mean depth of } \\
\text { clusters }\end{array}$ & $\begin{array}{l}\text { Standard } \\
\text { deviation of } \\
\text { cluster depth }\end{array}$ & $\begin{array}{l}\text { Number of clusters } \\
\text { with depth greater } \\
\text { than } 5\end{array}$ & $\begin{array}{l}\text { Mean depth of } \\
\text { clusters with depth } \\
\text { greater than } 5\end{array}$ & $\begin{array}{l}\text { Standard deviation } \\
\text { of clusters with } \\
\text { depth greater than } \\
5\end{array}$ \\
\hline 16640 & 21,256 & 43.61 & 127.54 & 18,198 & 50.62 & 136.60 \\
\hline $72638 \quad 22$ & 25,837 & 54.66 & 219.37 & 22,289 & 63.05 & 235.10 \\
\hline 82121_15 & 26,882 & 66.65 & 279.28 & 23,015 & 77.53 & 300.47 \\
\hline AsNiN2 & 22,793 & 38.14 & 189.94 & 18,847 & 45.70 & 208.09 \\
\hline AsOk3 & 17,057 & 24.22 & 131.27 & 12,610 & 32.08 & 151.89 \\
\hline bar22 & 18,745 & 28.32 & 88.47 & 14,987 & 34.93 & 97.83 \\
\hline bar06 & 20,640 & 39.36 & 160.01 & 17,346 & 46.47 & 173.64 \\
\hline JC6731B11 & 20,340 & 40.74 & 148.45 & 17,359 & 47.39 & 159.75 \\
\hline $\mathrm{NeIn} 2$ & 6,133 & 12.25 & 25.82 & 3,706 & 19.07 & 31.39 \\
\hline $\mathrm{NeOg} 1$ & 6,055 & 12.36 & 18.57 & 3,797 & 18.61 & 21.09 \\
\hline SEPR3 & 11,611 & 12.73 & 24.74 & 7,380 & 18.96 & 29.25 \\
\hline VuMaU1 & 20,884 & 35.76 & 95.06 & 17,346 & 42.65 & 102.96 \\
\hline VuTO2 & 10,607 & 18.50 & 59.61 & 7,508 & 25.33 & 69.71 \\
\hline
\end{tabular}

pyRAD clustering (c85)

\begin{tabular}{|c|c|c|c|c|c|c|}
\hline ID sample & $\begin{array}{l}\text { Total number } \\
\text { of clusters }\end{array}$ & $\begin{array}{l}\text { Mean depth of } \\
\text { clusters }\end{array}$ & $\begin{array}{l}\text { Standard } \\
\text { deviation of } \\
\text { cluster depth }\end{array}$ & $\begin{array}{l}\text { Number of clusters } \\
\text { with depth greater } \\
\text { than } 5\end{array}$ & $\begin{array}{l}\text { Mean depth of } \\
\text { clusters with depth } \\
\text { greater than } 5\end{array}$ & $\begin{array}{l}\text { Standard deviation } \\
\text { of clusters with } \\
\text { depth greater than } \\
5\end{array}$ \\
\hline 16640 & 19,884 & 44.17 & 101.89 & 17,283 & 50.52 & 107.86 \\
\hline $72638 \_22$ & 24,008 & 57.15 & 228.03 & 21,070 & 64.84 & 242.41 \\
\hline $82121 \quad 15$ & 24,580 & 69.94 & 282.61 & 21,537 & 79.55 & 300.68 \\
\hline AsNiN̄ 2 & 21,056 & 39.13 & 191.94 & 17,750 & 46.05 & 208.32 \\
\hline AsOk3 & 15,728 & 24.50 & 105.61 & 11,915 & 31.73 & 120.45 \\
\hline bar22 & 17,698 & 28.61 & 83.40 & 14,291 & 34.96 & 91.68 \\
\hline bar06 & 19,441 & 40.50 & 155.99 & 16,580 & 47.15 & 168.02 \\
\hline JC6731B11 & 19,045 & 41.52 & 139.60 & 16,444 & 47.77 & 149.28 \\
\hline NeIn 2 & 5,848 & 12.63 & 27.34 & 3,600 & 19.35 & 33.10 \\
\hline $\mathrm{NeOg} 1$ & 5,831 & 12.76 & 19.88 & 3,712 & 18.99 & 22.66 \\
\hline SEPR3 & 11,244 & 12.81 & 20.59 & 7,195 & 18.96 & 23.60 \\
\hline VuMaU1 & 19,413 & 36.77 & 94.33 & 16,375 & 43.23 & 101.40 \\
\hline VuTO2 & 10,045 & 18.81 & 59.55 & 7,194 & 25.49 & 69.23 \\
\hline
\end{tabular}

\begin{tabular}{|c|c|c|c|c|c|c|}
\hline & & & pyRA & D clustering (c90) & & \\
\hline ID sample & $\begin{array}{l}\text { Total number } \\
\text { of clusters }\end{array}$ & $\begin{array}{l}\text { Mean depth of } \\
\text { clusters }\end{array}$ & $\begin{array}{l}\text { Standard } \\
\text { deviation of } \\
\text { cluster depth }\end{array}$ & $\begin{array}{l}\text { Number of clusters } \\
\text { with depth greater } \\
\text { than } 5\end{array}$ & $\begin{array}{l}\text { Mean depth of } \\
\text { clusters with depth } \\
\text { greater than } 5\end{array}$ & $\begin{array}{l}\text { Standard deviation } \\
\text { of clusters with } \\
\text { depth greater than } \\
5\end{array}$ \\
\hline 16640 & 19,181 & 44.74 & 103.33 & 16,828 & 50.72 & 108.99 \\
\hline $72638 \_22$ & 23,039 & 57.73 & 211.75 & 20,417 & 64.89 & 223.93 \\
\hline $82121 \_15$ & 23,544 & 71.10 & 287.50 & 20,872 & 79.96 & 304.21 \\
\hline AsNiN2 & 20,266 & 39.13 & 174.49 & 17,184 & 45.78 & 188.72 \\
\hline AsOk3 & 15,095 & 24.79 & 106.79 & 11,585 & 31.71 & 121.05 \\
\hline bar22 & 17,166 & 28.80 & 84.49 & 13,943 & 35.00 & 92.65 \\
\hline bar06 & 18,912 & 41.06 & 158.41 & 16,206 & 47.59 & 170.25 \\
\hline JC6731B11 & 18,387 & 41.72 & 137.37 & 16,015 & 47.61 & 146.27 \\
\hline NeIn2 & 5,679 & 12.66 & 25.03 & 3,542 & 19.18 & 29.85 \\
\hline $\mathrm{NeOg} 1$ & 5,692 & 12.95 & 20.34 & 3,661 & 19.11 & 23.16 \\
\hline SEPR3 & 11,021 & 12.86 & 19.55 & 7,089 & 18.95 & 22.13 \\
\hline VuMaU1 & 18,692 & 37.02 & 94.83 & 15,904 & 43.16 & 101.57 \\
\hline VuTO2 & 9,761 & 19.05 & 60.22 & 7,043 & 25.65 & 69.78 \\
\hline
\end{tabular}


Table S8. RAD-seq matrices statistics

\begin{tabular}{|c|c|c|c|c|c|c|c|}
\hline $\begin{array}{l}\text { RAD-seq } \\
\text { matrix }\end{array}$ & Number of loci & $\begin{array}{l}\text { Number of } \\
\text { base pairs }\end{array}$ & $\begin{array}{l}\text { Percentage of } \\
\text { missing data }\end{array}$ & $\begin{array}{l}\text { Number of } \\
\text { variable sites }\end{array}$ & $\begin{array}{l}\text { Percentage of } \\
\text { variable sites }\end{array}$ & $\begin{array}{l}\text { Number of } \\
\text { parsimony } \\
\text { informative } \\
\text { sites }\end{array}$ & $\begin{array}{l}\text { Percentage of } \\
\text { parsimony } \\
\text { informative } \\
\text { sites }\end{array}$ \\
\hline $\mathrm{c} 80 \mathrm{~d} 5 \mathrm{~m} 4 \mathrm{p} 3$ & 15,331 & $1,303,485$ & 51.15 & 121,475 & 9.32 & 37,722 & 2.89 \\
\hline $\mathrm{c} 80 \mathrm{~d} 5 \mathrm{~m} 6 \mathrm{p} 3$ & 9,824 & 835,710 & 43.18 & 85,987 & 10.29 & 29,077 & 3.48 \\
\hline $\mathrm{c} 80 \mathrm{~d} 5 \mathrm{~m} 8 \mathrm{p} 3$ & 4,039 & 343,583 & 33.12 & 39,877 & 11.61 & 14,084 & 4.10 \\
\hline $\mathrm{c} 80 \mathrm{~d} 5 \mathrm{~m} 10 \mathrm{p} 3$ & 675 & 57,406 & 20.91 & 7,564 & 13.18 & 2,423 & 4.22 \\
\hline $\mathrm{c} 85 \mathrm{~d} 5 \mathrm{~m} 4 \mathrm{p} 3$ & 15,499 & $1,314,995$ & 51.62 & 109,124 & 8.30 & 35,303 & 2.68 \\
\hline$c 85 \mathrm{~d} 5 \mathrm{~m} 6 \mathrm{p} 3$ & 9,766 & 828,960 & 43.54 & 76,353 & 9.21 & 26,955 & 3.25 \\
\hline $\mathrm{c} 85 \mathrm{~d} 5 \mathrm{~m} 8 \mathrm{p} 3$ & 3,884 & 329,620 & 33.37 & 33,894 & 10.28 & 12,689 & 3.85 \\
\hline $\mathrm{c} 85 \mathrm{~d} 5 \mathrm{~m} 10 \mathrm{p} 3$ & 618 & 52,373 & 21.17 & 5,984 & 11.43 & 2,041 & 3.90 \\
\hline $\mathrm{c} 90 \mathrm{~d} 5 \mathrm{~m} 4 \mathrm{p} 3$ & 15,595 & $1,318,652$ & 52.78 & 89,781 & 6.81 & 29,801 & 2.26 \\
\hline c90d5m6p3 & 9,310 & 787,167 & 44.29 & 59,659 & 7.58 & 21,828 & 2.77 \\
\hline c90d5m8p3 & 3,396 & 286,979 & 33.74 & 23,903 & 8.33 & 9,559 & 3.33 \\
\hline c90d5m10p3 & 481 & 40,605 & 21.38 & 3,592 & 8.85 & 1,357 & 3.34 \\
\hline
\end{tabular}




\title{
CHAPTER 4
}

\section{No evidence of seamount-driven isolation in deep-sea hydrothermal vent barnacle populations}

\begin{abstract}
Patterns of spatial genetic population structuring provide insight into the factors that limit dispersal and connectivity of species. Deep-sea hydrothermal vents are the focus of increasing interest for the mining of mineral resources found in polymetallic sulphide deposits. Understanding the genetic diversity and population connectivity of vent species is critical for assessing the potential effects of mining on these ecosystems. Population genetic studies of vent species have mainly focused on mid-ocean ridge systems and back-arc spreading centers. However, vents also occur in active seamounts worldwide. Seamounts are hypothesized to behave as isolated island-like systems, where population connectivity is limited and endemicity is promoted (seamount endemicity hypothesis). In this study, we aim to test this seamount endemicity hypothesis using novel genome-wide restriction-site associated DNA (RAD) sequence data from three hydrothermal vent barnacle species. Comparisons of the genetic diversity and population structuring patterns of barnacle populations from seamounts and spreading ridges revealed patterns of population genetic structuring that do not conform to the predictions from the seamount endemicity hypothesis. The patterns of genetic variation among individuals collected from seamounts and spreading ridges, separated horizontally by hundreds of kilometers and vertically by hundreds of meters, did not reject the null hypothesis of panmixia within each species. These inferences are largely insensitive the to de novo assembly parameters used to identify loci from sequence reads. We suggest that the seamount endemicity hypothesis warrants further testing using high-resolution genetic markers in other vent organisms with differing life history strategies (e.g., brooders) that may limit their dispersal potential, as well as in non-vent organisms, which are not exposed to evolutionary pressures imposed by the dynamic nature of hydrothermal vent systems.
\end{abstract}




\section{INTRODUCTION}

Patterns of spatial genetic population structuring provide insight into the factors that limit dispersal and connectivity throughout a species' range. Empirical evidence has revealed the fundamental importance of intrinsic and extrinsic factors, such as habitat discontinuity (Reitzel et al. 2013; D'Aloia et al. 2014), depth (Prada \& Hellberg 2013), currents (Kelly \& Palumbi 2010; White et al. 2010), distance (Alberto et al. 2011), larval developmental mode (Kelly \& Palumbi 2010), and symbiotic associations (Beinart et al. 2012), acting as barriers to gene flow in marine species and ultimately driving biodiversity patterns in the ocean. Understanding how populations are spatially and temporally interconnected is critical due to the need for marine biodiversity and ecosystems protection against increasing threats created by human activities (Christensen et al. 1996).

Deep-sea hydrothermal vents are the focus of increasing interest for the mining of mineral resources found in polymetallic sulphide deposits (Boschen et al. 2013), which form by precipitation during mixing of metal-rich vent fluids with bottom seawater. Deep-sea hydrothermal vents (hereafter simply referred to as vents) host some of the most spectacular and unique ecosystems on earth that thrive on in situ primary productivity derived from chemosynthesis. Vents present a sharp contrast to other ecosystems in the deep sea, due to their marked patchiness, extremely steep chemical and thermal gradients over centimeter scales, and relatively high frequency of disturbances given their occurrence on highly dynamic geological settings (Van Dover 2000). Consequently, vent environments present extreme selective pressures, evolutionary speaking, and are characterized by their low biodiversity and high endemicity. These characteristics make vent ecosystems potentially susceptible to disturbances caused by deep-sea mining. Although vent organisms have adapted to the natural dynamics and ephemerality of hydrothermal vents, the potential disturbances from mining are likely to have multiplicative harmful effects at unprecedented scales (Van Dover 2010). Understanding the genetic diversity and population connectivity of vent species is critical to assessments of the potential effects of mining on these ecosystems.

Population genetic studies of vent species from spreading ridge systems commonly reveal patterns of genetic diversity consistent with high gene flow along ridge axes extending for hundreds to thousands of kilometers, yet factors such as depth and ocean currents have been identified as barriers to dispersal at regional scales (see review by Vrijenhoek (2010)). The geomorphology of spreading ridges is known to modify local current regimes and generate significant current flows along ridge axes (Thurnherr et al. 2011; Lavelle et al. 2012), which are hypothesized to facilitate dispersal among spatially separated vent fields over long distances. Deep-sea hydrothermal vents also occur on active volcanic seamouts located 
on hotspots or island arcs. However, the population structuring and genetic connectivity dynamics in these systems are much less understood.

Seamounts are hypothesized to behave as isolated island-like systems, where population connectivity, and therefore gene flow, are limited (commonly referred to as the 'seamount endemicity hypothesis', de Forges et al. 2000). Such isolation might arise due to the patchiness of the seamounts habitats (particularly of active seamounts as most seamounts are inactive), combined with larval behaviors, physical oceanographic phenomena, such as Taylor columns, that could cause larvae retention around seamount summits (Wilson \& Kaufmann 1986; Mullineaux \& Mills 1997; de Forges et al. 2000; Metaxas 2011), or a combination of these factors. The seamount endemicity hypothesis predicts that there will be relatively high levels of endemicity of genetic variants and significant structure among populations from different seamounts. Consistent with this hypothesis Smith et al. (2004) found heterogeneity in allozyme frequencies and significant genetic differentiation between populations of Bathymodiolus vent mussels from two active seamounts of the Kermadec arc. Contrastingly, Tunnicliffe et al. (2010) and Watanabe et al. (2005) found significant differentiation in mitochondrial sequences between volcanic arc basins, but no differentiation among seamount populations of vent flatfish and barnacle species within each arc basin. Other studies in non-vent seamount fauna have found similar patterns of absence of population structuring among seamount populations of deep-sea corals (Thoma et al. 2009), clams (Clague et al. 2012), and ophiuroids (Cho \& Shank 2010), when examining mitochondrial markers. However, the mitochondrion has a intermediate mutation rate compared to other autosomal markers such as microsatellites and single nucleotide polymorphisms (SNPs), and mounting evidence indicates that significant amounts of genetic variability in populations and species can go undetected based solely on mitochondrial data (Emerson et al. 2010; Herrera \& Shank Chapter 5). For example, a study on a species of Patagonian toothfish revealed significant genetic structure between eastern and western Southern Ocean populations using data from microsatellites, when a lack of genetic structure had been previously concluded using mitochondrial data only (Rogers et al. 2006). Therefore, interpretations of nonendemicity in seamounts based on mitochondrial DNA data have been challenged (Baco \& Cairns 2012).

In this study we aim to test the seamount endemicity hypothesis in deep-sea hydrothermal-vent fauna using novel data from thousands of genome-wide SNPs obtained from restriction-site associated DNA (RAD) markers (Baird et al. 2008). Our approach is to compare the genetic diversity and population structuring patterns of seamount and spreading ridge populations in three hydrothermal vent barnacle species: 1) Vulcanoleapas scotiaensis from East Scotia ridge; 2) Vulcanolepas osheai from the Kermadec arc; and 3) Eochionelasmus ohtai from the Lau Basin. Under the seamount endemicity hypothesis, we 
expect to find higher population genetic structuring between seamount populations than between populations from spreading ridges.

\section{METHODS}

Barnacle pecimens were collected from deep-sea hydrothermal vents at 2-3 localities each within the East Scotia ridge (E2 and E9 vent sites), the Kermadec arc (Healy and Tangaroa seamounts), and the Lau Basin (Tahi Moana vent site, and the Mata Ua and Founalei South seamounts). Morphological identifications were performed on using stereo-microscopy and species descriptions as references (Table 1, Fig. 1). Species were identified as Vulcanoleapas scotiaensis, Vulcanolepas osheai, and Eochionelasmus ohtai respectively. Species identifications of representative specimens were confirmed through multilocus phylogenetic analyses by Herrera et al. (Chapter 3).

Table 1. Collection information for the specimens used in this study.

$\begin{array}{llllrlll}\text { Species } & \text { Region } & \text { Population locality } & \text { Collection event } & \begin{array}{l}\text { Depth } \\ (\mathbf{m})\end{array} & \text { Lat. } & \text { Lon. } & \text { Collection ID } \\ & & & & 2700 & -56.06 & -30.33 \text { WHOI-NEOE2 } \\ \text { Vulcanolepas scotiaensis } & \text { East Scotia ridge } & \text { E2 vent field } & \text { JC042 } & \text { JC042 } & 2400 & -60.05 & -29.93 \text { WHOI-NEOE9 } \\ \text { Vulcanolepas scotiaensis } & \text { East Scotia ridge } & \text { E9 vent field } & \text { TAN1104/073 } & 1255 & -35.01 & 178.98 \text { NIWA-72638 } \\ \text { Vulcanolepas osheai } & \text { Kermadec arc } & \text { Healy volcano } & \text { TAN1206/017 } & 682 & -36.32 & 178.03 \text { NIWA-82121 } \\ \text { Vulcanolepas osheai } & \text { Kermadec arc } & \text { Tangaroa volcano } & \text { Tahi Moana vent field } & \text { TN236/J2-444 } & 2232 & -20.68 & -176.18 \text { WHOI-13288 } \\ \text { Eochionelasmus ohtai } & \text { Lau Basin } & \text { Founalei South volcano } & \text { RR1211/Q4K-323 } & 956 & -17.54 & -174.58 \text { WHOI-20665 } \\ \text { Eochionelasmus ohtai } & \text { Lau Basin } & \text { Mata Ua volcano } & \text { RR1211/Q4K-328 } & 2391 & -15.02 & -173.79 \text { WHOI-20791 } \\ \text { Eochionelasmus ohtai } & \text { Lau Basin } & & & & \end{array}$

\section{Molecular laboratory methods}

Total genomic DNA was purified from specimens by: (1) digesting the tissue in 2\% CTAB buffer (Teknova) with proteinase K and RNAse A/T1 (Fermentas) for 1 hour, (2) separating nucleic acids with chloroform: isoamyl alcohol (24:1) (Fermentas) and phenol: chloroform: isoamyl alcohol (25:24:1, Tris buffered at $\mathrm{pH} 8.0$ ) (Fermentas), (3) precipitating nucleic acids with 100\% ethanol (1:1 volume ratio), and (4) washing the precipitate twice with $70 \%$ ethanol.

Concentration-normalized genomic DNA was submitted to Floragenex Inc. (Eugene, OR) for library preparation and RAD sequencing. In short, the RAD sequencing method consists of: 1) the digestion of genomic DNA for each individual with a restriction enzyme; 2) ligation of the resulting fragments to sequencing adapters with unique barcodes for each individual; 3) size-selection and enrichment of the 
fragments successfully ligated to the adapters; and 4) sequencing via a high-throughput platform. Individual libraries for Vulcanolepas osehai and Eochionelasmus ohtai were produced from DNA digested with a high-fidelity SbfI restriction enzyme, which is predicted to cut between 5,000 and 15,000 times in the genome of a thoracican barnacle (Table S1) (Herrera et al. Chapter 3). Libraries for Vulcanolepas scotiaensis were produced from DNA digested with a high-fidelity SgrAI restriction enzyme, which is predicted to cut between 20,000 and 60,000 times in the genome of a thoracican barnacle. The cut-frequency ranges for both SbfI and SgrAI were predicted by the observed recognition sequence frequencies and their estimated probability using trinucleotide composition models in the genome of the crustacean Daphnia pulex using the software predRAD (Herrera et al. Chapter 2). Ranges of genome size for barnacles were obtained from the Animal Genome Size Database (http://ww.genomesize.com). Barcode tags were 10-base pairs long. Libraries were sequenced by multiplex on an Illumina Hi-Seq 2000 sequencer.
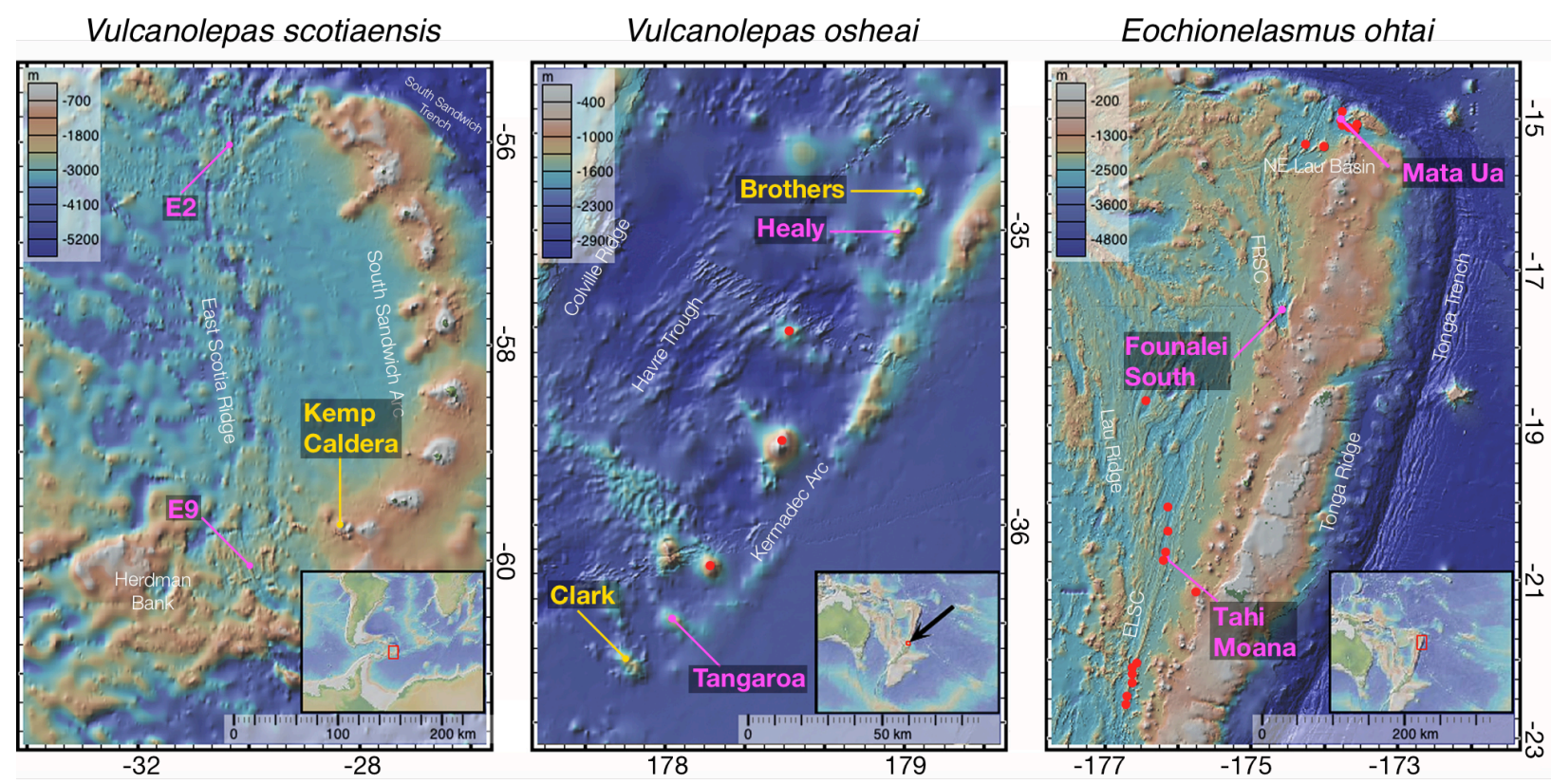

Figure 1. Geographic location of vent barnacle populations for each species. Bathymetry maps show the specific areas where samples have been obtained. Purple labels indicate locations of collections of populations included in this study, but marked to provide context. Yellow labels indicate locations of known populations not included in this study for context. Red dots indicate locations of other active hydrothermal vent sites. Color scale indicates corresponding depths in meters. Bottom right bars indicate distance scale in kilometers. Small maps inset within the bottom right of each subplot indicate region location in a global perspective. Base bathymetry maps were generated using the program GeoMapApp (http://www.geomapapp.org) with data from the Global Multi-Resolution Topography (GMRT) Synthesis (Ryan et al. 2009). 


\section{Data filtering}

Sequence reads were de-multiplexed and quality filtered with the process_radtags program from the package Stacks v1.19 (Catchen et al. 2013b). Barcodes and Illumina adapters were excluded from each read, and length was truncated to 70bp (-t 70). Reads with ambiguous bases were discarded $(-c)$. Reads with an average quality score below $10(-s$ 10) within a sliding window of $15 \%$ of the read length (-w $0.15)$ were discarded $(-q)$. The rescue barcodes and RAD-tags algorithm was enabled $(-r)$.

\section{Tests of parameters for de novo loci assembly}

To explore the effects of parameter choice on de novo assemblies of RAD loci using the denovo_map pipeline in Stacks, we tested an array of parameters following the guidelines and modified R scripts by Mastretta-Yanes et al. (2014). For each species dataset we independently performed multiple de novo loci assemblies modifying the following individual parameter values, while keeping default values for the rest: the minimum number of identical raw reads required to create a stack of identical unique sequences for each individual (- $m$ 2, 4, 6, 8, 10 for $V$. scotiaensis; and $-m$ 2-8, 10, and 12 for $V$. osheai and E. ohtai), the maximum number of stacks at a single locus for each individual (--max_locus_stacks 2-5 for $V$. scotiaensis; and --max_locus_stacks 2-6 for V. osheai and E. ohtai), the number of mismatches allowed between loci for each individual (-M 2, 3, 4 for $V$. scotiaensis; and -M 2-6, 8 for V. osheai and E. ohtai), and the number of mismatches allowed between loci when building the catalog of all loci in a species $(-n$ 0-4 for V. scotiaensis; and -n 0-5 for V. osheai and E. ohtai). High-confidence SNP calls in STACKS are performed using a maximum-likelihood framework that accounts for sequencing error and variable depth of coverage among loci (Hohenlohe et al. 2010; Catchen et al. 2013b). The results from each assembly were loaded onto a MySQL database and indexed through the load_radtags and index_radtags tools.

We calculated the number of reads and loci coverage per individual from each assembly, and filtered the data to exclude individuals having less than $50 \%$ of the mean number of loci per individual and keep only loci present in at least $80 \%$ of the individuals. We executed the populations program of Stacks after each de novo assembly using only individuals and loci that passed the aforementioned filter (whitelist $-W$ ). To explore the influence of different assembly parameters on population differentiation estimates, we calculated population $\mathrm{F}_{\mathrm{ST}}$ values from SNPs utilizing a $p_{-}$value filter ( $f$ ) to keep only significant estimates $(\alpha=0.05)$. To examine the effect of different assembly parameters on the inferred genetic variability within and among populations, we calculated Euclidean distances among individuals from exported SNP data in plink format, and performed Neighbor Joining similarity and principal component analyses in R. Optimal de novo assembly parameter settings were chosen conservatively, aiming to 
maximize the number of SNPs recovered while minimizing the within-population genetic distances, following guidelines by Mastretta-Yanes et al. (2014).

\section{Demographic inferences}

We performed de novo assemblies of RAD loci using optimal parameter settings for each species in Stacks, as explained above. To estimate population genetic descriptive statistics per SNP (genetic diversity $\pi$, proportion of polymorphic loci, observed heterozygosity, minor allele frequency, number of private alleles, inbreeding index $\mathrm{F}_{\mathrm{IS}}$, and population differentiation index $\mathrm{F}_{\mathrm{ST}}$ ) we executed the populations program of Stacks, using only individuals and loci that passed aforementioned filters. We only analyzed loci that were present in all populations of each species $(-p)$ and in at least $75 \%$ of individuals per population $(-r)$. As before, we calculated population $\mathrm{F}_{\mathrm{ST}}$ values from SNPs utilizing a $p \_$value filter $(-f)$ correction $(\alpha=0.05)$. We exported SNP data in plink format, keeping only one SNP per loci to avoid violating the assumption of independence among markers. To detect possible population structuring we constructed Neighbor Joining similarity dendrograms and performed principal component analyses with data from individuals for each species, as described above. To explore possible non-equilibrium signals in populations we examined frequency distribution plots of minor allele frequencies and inbreeding indices $\left(\mathrm{F}_{\mathrm{IS}}\right)$ in $\mathrm{R}$.

\section{RESULTS}

\section{Sequencing results}

We generated RAD-seq data for 117 individuals from three species of vent barnacles: 28 individuals from two populations of Vulcanolepas scotiaensis, E2 and E9, from the East Scotia rise; 36 individuals from two populations of Vulcanolepas osheai, Healy and Tangaroa, from the Kermadec arc; and 53 individuals from three populations of Eochionelasmus ohtai, Tahi Moana, Founalei South, and Mata Ua, from the Lau Basin (Table 2). We obtained approximately $3.1 \pm 1.5$ (mean \pm standard deviation) million reads per individual for $V$. scotiaensis, with individual values ranging from 1.1 to 7.2 million reads. For $V$. osheai we obtained approximately $1.4 \pm 0.4$ million reads per individual, with individual values ranging from 0.8 to 2.6 million reads. Lastly, for E. ohtai we obtained approximately $2.3 \pm 0.9$ million reads per individual, with individual values ranging from 0.8 to 4.8 million reads. Overall, more than $88 \%$ of sequence reads were retained after quality filtering. 
Table 2. RAD sequencing results, filtering and de novo assembly statistics. $\mathrm{N}$ indicates the number of specimens per population. The numbers of raw and retained reads are shown as population mean \pm standard deviation.

$\begin{array}{lllrrrr}\text { Species } & \text { Population } & \text { Sequence IDs } & \text { N } & \text { Raw reads } & \text { Retained reads } \begin{array}{c}\text { \% reads } \\ \text { retained }\end{array} \\ \text { Vulcanolepas scotiaensis } & \text { E2 } & \text { NEO_*E2 } & 14 & 3,531,674 \pm 1,490,665 & 3,058,341 \pm 1,086,450 & 88 \pm 6 \\ \text { Vulcanolepas scotiaensis } & \text { E9 } & \text { NEO_*E9 } & 14 & 2,744,428 \pm 1,441,426 & 2,369,012 \pm 1,087,019 & 88 \pm 6 \\ \text { Vulcanolepas } \text { osheai } & \text { Healy } & 72638_{-}^{*} & 18 & 1,254,447 \pm 245,024 & 1,134,690 \pm 249,516 & 90 \pm 6 \\ \text { Vulcanolepas } \text { osheai } & \text { Tangaroa } & 82121_{-}^{*} & 18 & 1,500,636 \pm 424,632 & 1,370,937 \pm 418,941 & 91 \pm 5 \\ \text { Eochionelasmus ohtai } & \text { Tahi Moana } & 13288_{-}^{*} & 17 & 2,170,591 \pm 906,494 & 1,978,867 \pm 876,981 & 90 \pm 5 \\ \text { Eochionelasmus ohtai } & \text { Founalei South } & \text { EoFo_* } & 18 & 2,438,023 \pm 728,551 & 2,322,302 \pm 742,900 & 95 \pm 3 \\ \text { Eochionelasmus ohtai } & \text { Mata Ua } & \text { EoMaU_* } & 18 & 2,167,746 \pm 1,180,479 & 2,147,705 \pm 1,161,193 & 91 \pm 5\end{array}$

* represents individual IDs

\section{De novo loci assembly parameter examination}

De novo loci assembly parameter variation tests produced between 18-55 thousand loci for $V$. scotiaensis containing on average 1.9-2.0 SNPs per locus (Fig. 2). These tests produced 4.1-5.5 and 10.5-13.0 thousand loci, containing on average 2.38-2.43 and 2.17-2.37 SNPs per locus, for $V$. scotiaensis and E. ohtai, respectively. Overall, the parameter controlling the minimum number of identical raw reads required to create a stack of identical unique sequences for each individual $(-m)$ had the largest influence on the number of markers obtained, particularly in the dataset from $V$. scotiaensis with a roughly linear decrease rate of 5 thousand loci per parameter unit increase (compared to a decrease rate of nearly 200 and 130 loci per parameter unit increase in $V$. osheai and $E$. ohtai, respectively). The parameter $-M$, which limits the number of mismatches allowed between loci for each individual, also produced a general decrease in the number of loci and SNPs as parameter value increased. The parameter $-n$, which limits the number of mismatches allowed between loci when building the catalog of all loci in a species, produced a similar decreasing effect to parameters $-m$ and $-M$, after the number of loci and SNPs peaked at $-n 1$. The parameter --max_locus_stacks, which limits the maximum number of stacks at a single locus for each individual, did not have a substantial effect on the number of loci or SNPs.

All individuals passed the imposed requirement of having more than $50 \%$ of the mean number of loci per individual per species. Mean $\mathrm{F}_{\mathrm{ST}}$ population differentiation values were consistently low, ranging from 0.0008 to 0.0020 (Fig. 3). Parameter $-n$ had the greatest influence in the mean $F_{\mathrm{ST}}$ values, which tended to increase and plateau with the parameter values (except in $V$. osheai, where mean $\mathrm{F}_{\mathrm{ST}}$ values oscillated). Increasing values of parameters $-M$ and $-m$ had opposite effects to parameter $-n$, causing a general drop in mean $\mathrm{F}_{\mathrm{ST}}$ values. Parameter --max_locus_stacks had negligible effects on the estimates of mean $\mathrm{F}_{\mathrm{ST}}$ values. 
a)

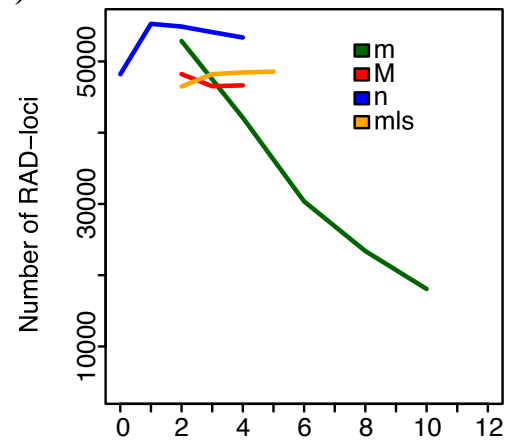

b)

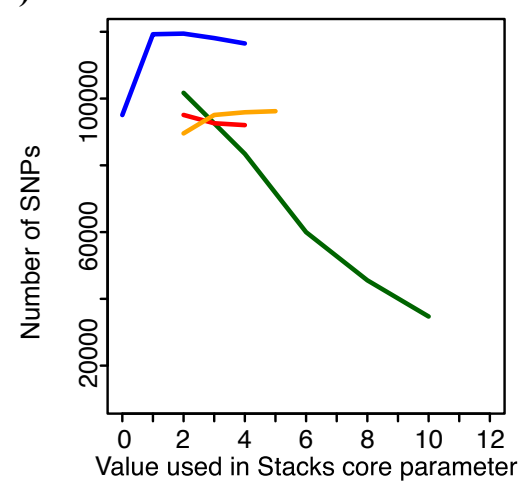

c)

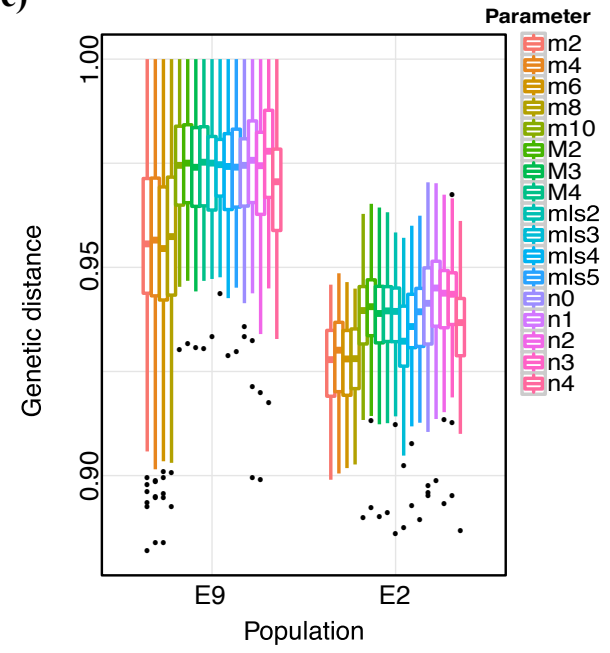

Vulcanolepas osheai

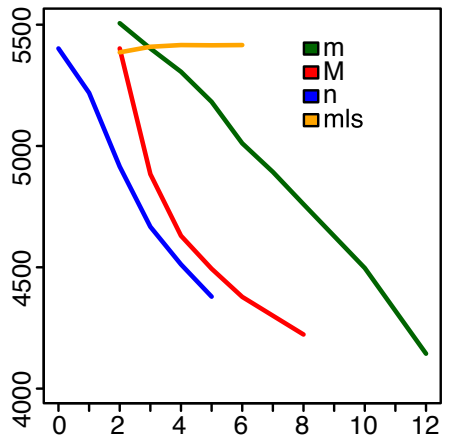

Value used in Stacks core parameter
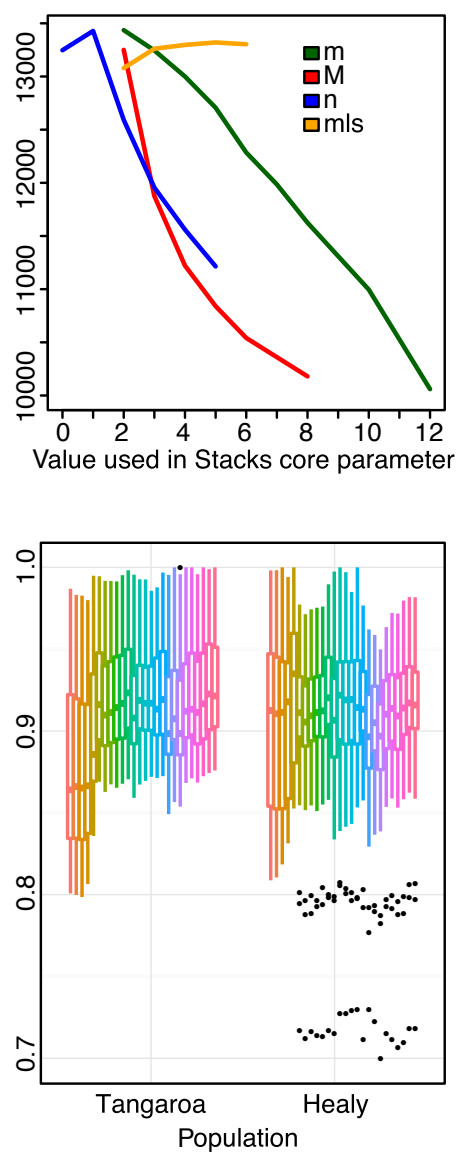

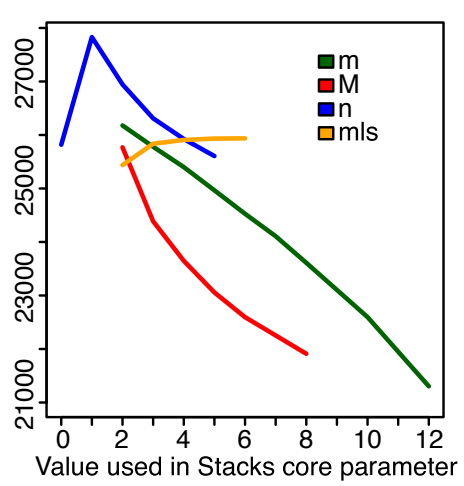

Eochionelasmus ohtai

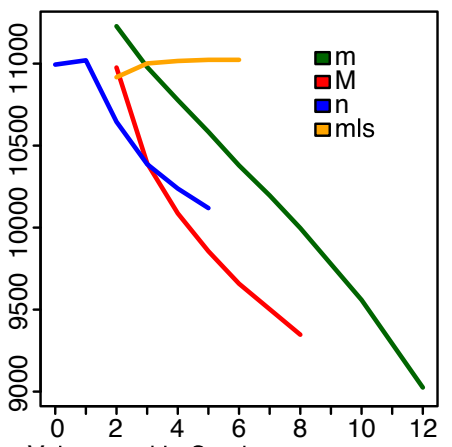

Value used in Stacks core parameter

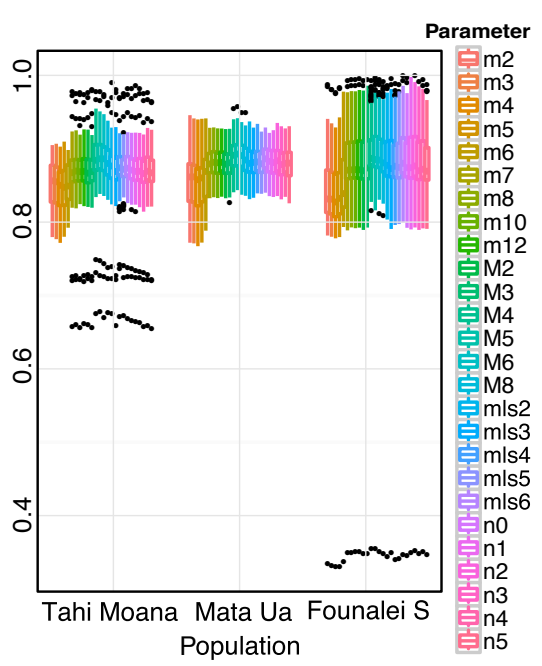

Figure 2. De novo loci assembly parameter influence on number of obtained loci, SNPs, and genetic distances for each vent barnacle dataset. Species are indicated at the top of each column. a) Plots of the value of each of the examined STACKS core parameters against the number of RAD-loci generated. b) Plots of the value of each of the examined STACKS core parameters against the number of SNPs generated. Line colors and legend indicate evaluated core parameters: the minimum number of identical raw reads required to create a stack of identical unique sequences for each individual ( $-m$ in green), the number of mismatches allowed between loci for each individual (- $M$ in red); the number of mismatches allowed between loci when building the catalog of all loci in a species (- $n$ in 
blue); and the maximum number of stacks at a single locus for each individual (--max_locus_stacks abbreviated as $\mathrm{mls}$ in yellow). c) Box plots of Euclidean genetic distances among individuals, per population, calculated using SNP data from each de novo loci assembly parameter examination. Colors and legend indicate evaluated core parameters.

Overall, Euclidean genetic distances among individuals within each population, calculated from SNPs in loci that were present in at least $80 \%$ of the individuals for each one of the different de novo loci assemblies, were markedly elevated (above 0.8) (Fig. 4). No significant differences in distance distributions were observed among populations or among loci assemblies using different parameters. Neighbor-Joining similarity dendrograms and principal component analyses performed with SNP data from each of the de novo loci assemblies were remarkably similar within species, and none of them revealed patterns indicative of population genetic structuring (Fig. 4).

Table 3. Summary statistics of de novo RAD stacks assembly. Stacks are defined as clusters of identical reads. N indicates the number of individuals per population. Values per population are shown as mean \pm standard deviation.

\begin{tabular}{llrlrlrr} 
Species & Population & N & $\begin{array}{l}\text { Number of } \\
\text { Stacks }\end{array}$ & \multicolumn{1}{l}{$\begin{array}{l}\text { Mean coverage } \\
\text { depth }\end{array}$} & $\begin{array}{l}\text { S.D. of coverage } \\
\text { depth }\end{array}$ & \multicolumn{1}{c}{$\begin{array}{l}\text { Maximum } \\
\text { coverage depth }\end{array}$} \\
Vulcanolepas scotiaensis & E2 & 14 & $99,758 \pm 10,413$ & $25 \pm 5$ & $23 \pm 7$ & $152 \pm 49$ & $28,284 \pm 11,158$ \\
Vulcanolepas scotiaensis & E9 & 14 & $85,349 \pm 20,098$ & $38 \pm 10$ & $130 \pm 56$ & $23,204 \pm 13,867$ \\
Vulcanolepas osheai & Healy & 18 & $28,533 \pm 3,869$ & $44 \pm 14$ & $139 \pm 34$ & $12,327 \pm 3,133$ \\
Vulcanolepas osheai & Tangaroa & 18 & $28,993 \pm 2,620$ & $65 \pm 24$ & $324 \pm 151$ & $37,552 \pm 20,832$ \\
Eochionelasmus ohtai & Tahi Moana & 17 & $28,074 \pm 2,449$ & $76 \pm 21$ & $386 \pm 125$ & $42,658 \pm 17,162$ \\
Eochionelasmus ohtai & Founalei South & 18 & $27,764 \pm 2,524$ & $71 \pm 33$ & $371 \pm 198$ & $42,108 \pm 25,723$
\end{tabular}

\section{De novo loci assembly with selected parameters}

We selected de novo assembly parameters conservatively, aiming to prevent pronounced losses of loci and SNPs while ensuring that there were enough reads to make high-confidence SNP calls $(-m>3)$, avoiding the formation of paralogs $(-M<4)$, avoiding calling fixed alleles as separate loci $(-n>0)$, and conforming to biological expectations (--max_locus_stacks $=2$, as usually no more than 2 alleles are found in SNP markers of diploid organisms). De novo loci assemblies with selected parameters ( $-m 4-M$ 3 -n 4 --max_locus_stacks 2) produced approximately $92 \pm 17$ thousand unique sequence stacks per individual of $V$. scotiaensis, with a mean coverage depth of $24 \pm 6 \mathrm{x}$ using the restriction enzyme SgrAI (Table 3). The restriction enzyme SbfI was used with both $V$. osheai and E. ohtai. Approximately $28 \pm 3$ thousand stacks per individual were produced for $V$. osheai, with a mean coverage depth of $41 \pm 13 \mathrm{x}$

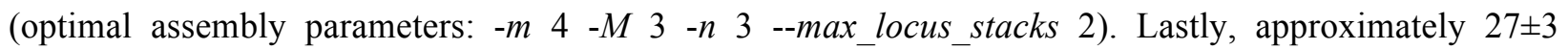


thousand stacks per individual were produced for E. ohtai, with a mean coverage depth of $73 \pm 28 \mathrm{x}$ (optimal assembly parameters: -m 5 -M 3 -n 3 --max_locus_stacks 2).
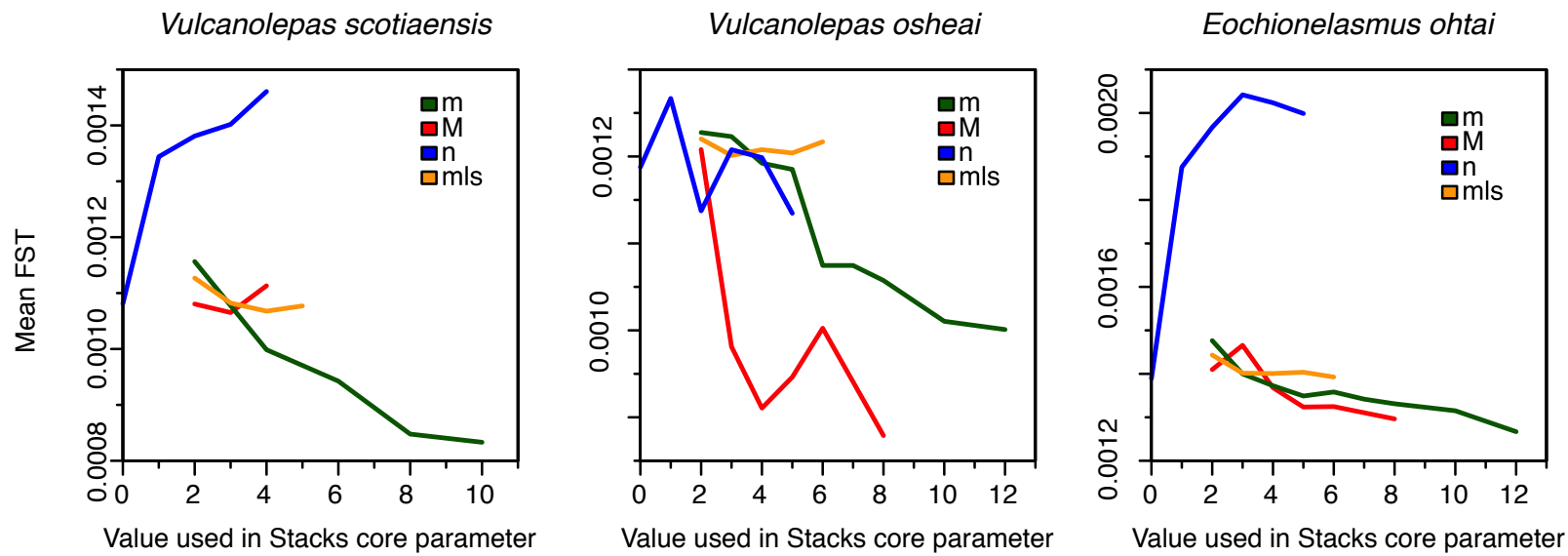

Figure 3. De novo loci assembly parameter influence on $\mathrm{F}_{\mathrm{ST}}$ estimation for vent barnacle datasets. Species are indicated at the top of each subplot. Plots show the value of each one of the examined STACKS core parameters against the estimated mean $\mathrm{F}_{\mathrm{ST}}$. Line colors and legend indicate evaluated core parameters: the minimum number of identical raw reads required to create a stack of identical unique sequences for each individual ( $m$ in green); the number of mismatches allowed between loci for each individual ( $M$ in red); the number of mismatches allowed between loci when building the catalog of all loci in a species ( $-n$ in blue); and the maximum number of stacks at a single locus for each individual (--max_locus_stacks, abbreviated as mls, in yellow).

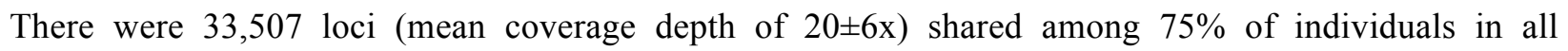
populations of $V$. scotiaensis, of which 28,270 contained one or more SNPs. Significantly fewer markers were obtained from $V$. osheai, with 4,384 loci (mean coverage depth of $34 \pm 11 \mathrm{x}$ ), but a larger fraction, 4,205, contained one or more SNPs. Lastly, there were 9,966 loci for E. ohtai (mean coverage depth of $60 \pm 22 x$ ), of which 9,430 contained one or more SNPs.

\section{Population demographic inferences}

Summary population genetic statistics revealed virtually identical levels of genetic diversity among populations of each species - in terms of percentage of polymorphic sites, mean observed heterozygosity and mean nucleotide diversity (Table 4). Overall, $V$. scotiaensis had the greatest genetic diversity, followed by $V$. osheai and E. ohtai. These patterns were maintained when summary statistics were calculated from variant positions alone, and from variant and fixed positions combined. 
Table 4. Population genetic statistics calculated only from variant positions, and from both variant and fixed positions. Values indicate means \pm standard deviation

\begin{tabular}{|c|c|c|c|c|c|c|c|}
\hline Population & $\begin{array}{l}\text { Private } \\
\text { alleles }\end{array}$ & $\begin{array}{l}\text { Variant } \\
\text { sites }\end{array}$ & $\begin{array}{l}\text { \% polym. } \\
\text { sites }\end{array}$ & $\begin{array}{l}\text { Major allele } \\
\text { frequency }\end{array}$ & $\begin{array}{l}\text { Observed } \\
\text { heterozygosity }\end{array}$ & $\begin{array}{l}\text { Nucleotide } \\
\text { diversity }(\pi)\end{array}$ & FIS \\
\hline E2 & 21,327 & 55,411 & 64.63 & $0.9403 \pm 0.0949$ & $0.0880 \pm 0.1311$ & $0.0981 \pm 0.1257$ & $0.0504 \pm 0.2356$ \\
\hline E9 & 19,600 & 55,415 & 61.51 & $0.9411 \pm 0.0938$ & $0.0823 \pm 0.1245$ & $0.0973 \pm 0.1261$ & $0.0667 \pm 0.2544$ \\
\hline Healy & 3,446 & 10,274 & 63.29 & $0.9419 \pm 0.0970$ & $0.0671 \pm 0.1034$ & $0.0935 \pm 0.1285$ & $0.1263 \pm 0.3041$ \\
\hline Tangaroa & 3,772 & 10,274 & 66.46 & $0.9422 \pm 0.0949$ & $0.0723 \pm 0.1082$ & $0.0938 \pm 0.1257$ & $0.1058 \pm 0.2851$ \\
\hline Tahi Moana & 4,977 & 23,177 & 50.83 & $0.9503 \pm 0.0922$ & $0.0523 \pm 0.0938$ & $0.0800 \pm 0.1241$ & $0.1305 \pm 0.3071$ \\
\hline Founalei South & 4,849 & 23,167 & 50.09 & $0.9507 \pm 0.0917$ & $0.0555 \pm 0.0980$ & $0.0795 \pm 0.1241$ & $0.1078 \pm 0.2846$ \\
\hline Mata Ua & 5,274 & 23,161 & 52.48 & $0.9504 \pm 0.0917$ & $0.0523 \pm 0.0922$ & $0.0798 \pm 0.1229$ & $0.1336 \pm 0.3106$ \\
\hline
\end{tabular}

All positions (variant and fixed)

\begin{tabular}{|c|c|c|c|c|c|c|c|}
\hline Population & $\begin{array}{l}\text { Private } \\
\text { alleles }\end{array}$ & Sites & $\begin{array}{l}\text { \% polym. } \\
\text { sites }\end{array}$ & $\begin{array}{l}\text { Major allele } \\
\text { frequency }\end{array}$ & $\begin{array}{l}\text { Observed } \\
\text { heterozygosity }\end{array}$ & $\begin{array}{l}\text { Nucleotide } \\
\text { diversity }(\pi)\end{array}$ & FIS \\
\hline E2 & 21,327 & $1,728,859$ & 2.07 & $0.9981 \pm 0.0200$ & $0.0028 \pm 0.0283$ & $0.0031 \pm 0.0283$ & $0.0016 \pm 0.0436$ \\
\hline E9 & 19,600 & $1,728,867$ & 1.97 & $0.9981 \pm 0.0200$ & $0.0026 \pm 0.0265$ & $0.0031 \pm 0.0283$ & $0.0021 \pm 0.0469$ \\
\hline Healy & 3,446 & 382,282 & 1.70 & $0.9984 \pm 0.0173$ & $0.0018 \pm 0.0200$ & $0.0025 \pm 0.0265$ & $0.0034 \pm 0.0539$ \\
\hline Tangaroa & 3,772 & 382,279 & 1.79 & $0.9984 \pm 0.0173$ & $0.0019 \pm 0.0224$ & $0.0025 \pm 0.0265$ & $0.0028 \pm 0.0500$ \\
\hline Tahi Moana & 4,977 & 853,175 & 1.38 & $0.9986 \pm 0.0173$ & $0.0014 \pm 0.0173$ & $0.0022 \pm 0.0245$ & $0.0035 \pm 0.0548$ \\
\hline Founalei South & 4,849 & 853,168 & 1.36 & $0.9987 \pm 0.0173$ & $0.0015 \pm 0.0173$ & $0.0022 \pm 0.0245$ & $0.0029 \pm 0.0500$ \\
\hline Mata Ua & 5,274 & 853,151 & 1.42 & $0.9987 \pm 0.0173$ & $0.0014 \pm 0.0173$ & $0.0022 \pm 0.0245$ & $0.0036 \pm 0.0557$ \\
\hline
\end{tabular}

Mean pairwise $\mathrm{F}_{\mathrm{ST}}$ values indicate low differentiation among populations within each species $(0.0011$ in V. scotiaensis; 0.0011 in V. osheai; and in E. ohtai 0.0018 between Tahi Moana and Founalei S., 0.0021 between Tahi Moana and Mata Ua, and 0.0017 between Founalei S. and Mata Ua). Globally, neither Neighbor-Joining similarity dendrograms nor principal component analyzes of SNP data produced clustering patterns of genetic variation consistent with population genetic structuring within species. In all cases, none of the axes in the principal component analyses explained more than $7 \%$ of the observed variance in the SNP data.

Minor allele frequency spectra show that a majority of the alleles in each population have low frequencies (Fig. 5), as expected for stable populations near mutation-drift equilibrium. $F_{I S}$ distributions for all populations were centered on zero indicating random mating and lack of population-sub structuring (Fig. 6). A small fraction of $F_{I S}$ values were positive, including noticeable clusters of values near 1, could be indicative of markers falling on non-recombinant genomic regions, such as sex-determining regions 
(Catchen et al. 2013a). Both minor allele frequency spectra and $\mathrm{F}_{\text {IS }}$ distributions were remarkably similar among populations of each species.

a)
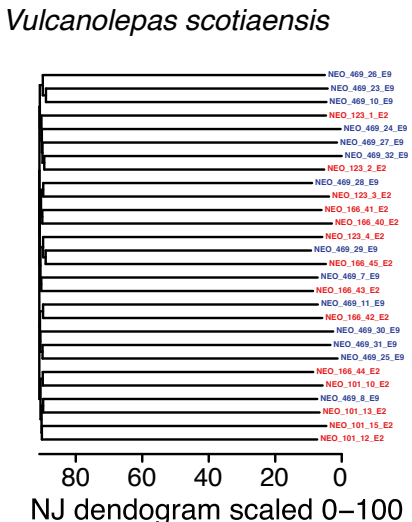

b)

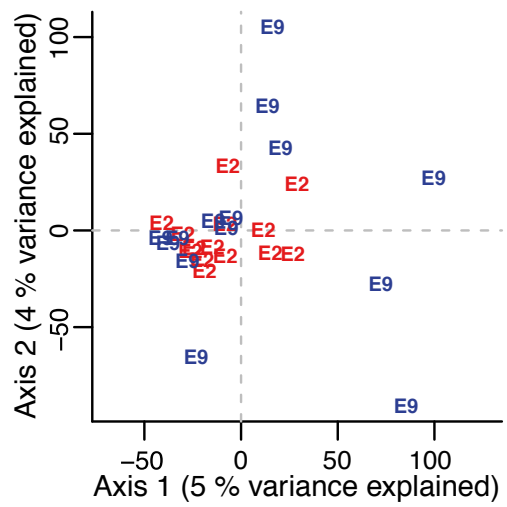

c)

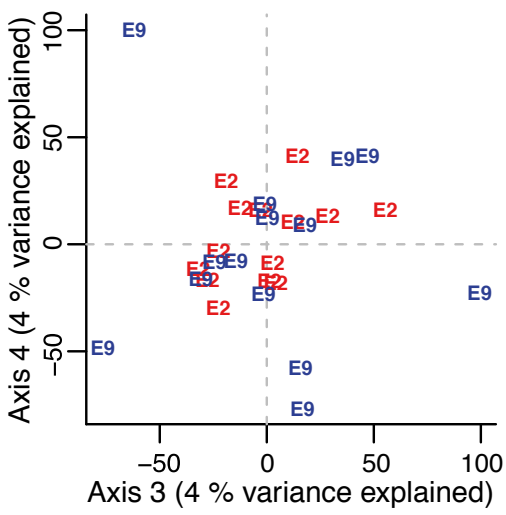

Vulcanolepas osheai
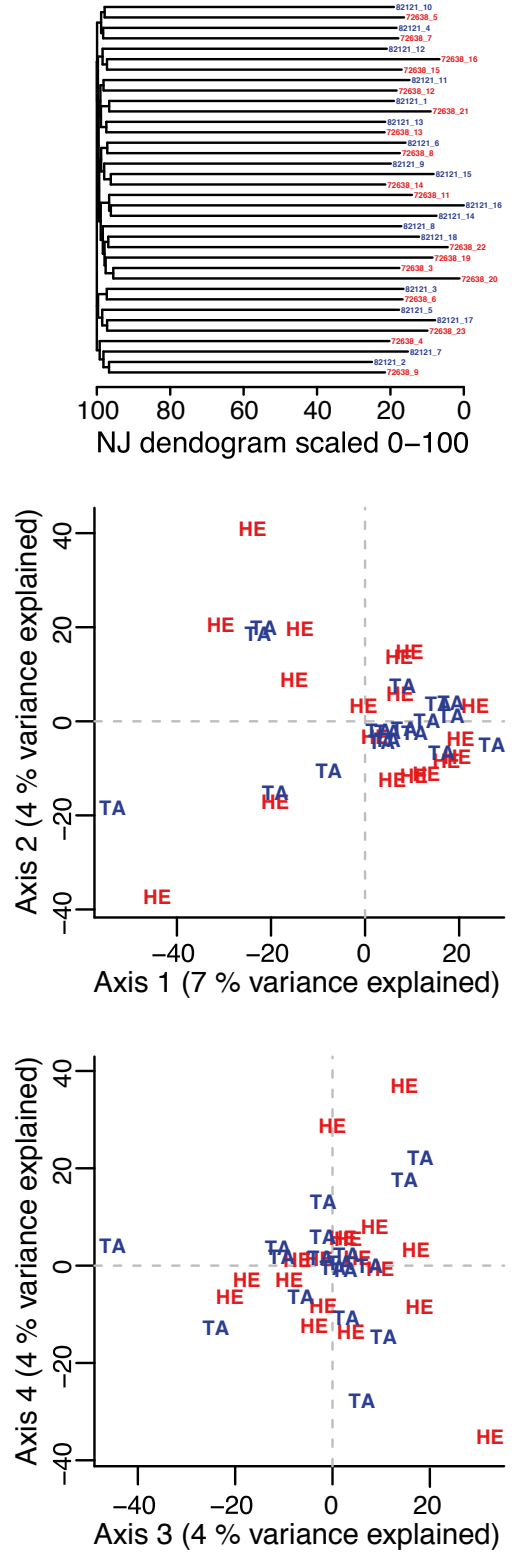

Eochionelasmus ohtai
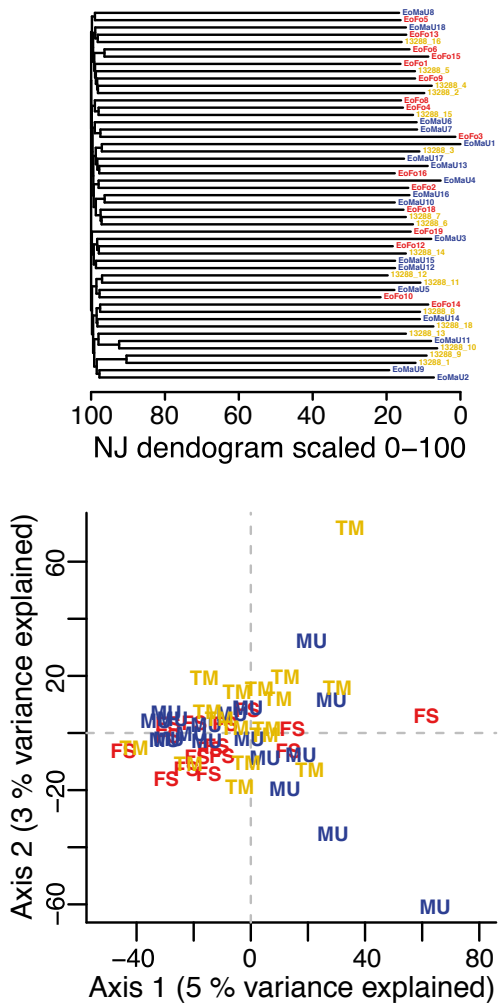

FS

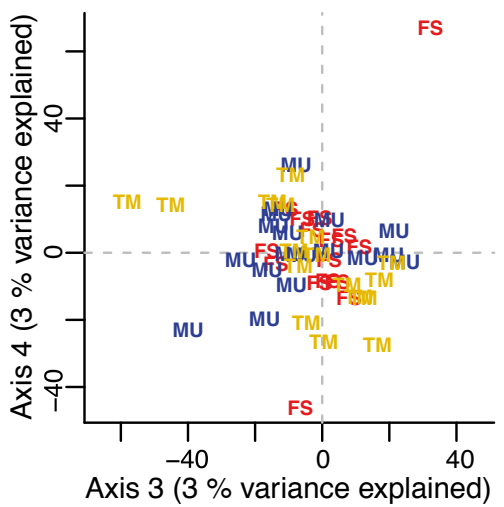

Figure 4. Genetic variability of SNP data from individuals for each vent barnacle species. Species are indicated in each column. a) Calibrated Neighbor-Joining dendrograms of genetic similarity from Euclidean distances among individuals per species. b) Plots of the first two axes of genetic variation among individuals per species found through principal component analyses. c) Plots of the third and fourth axes of genetic variation among individuals per species found through principal component analyses. Labels and colors indicate the source population per 
species: V. scotiaensis E2 in red and E9 in blue; V. osheai Healy (HE) in red and Tangaroa (TA) in blue; E. ohtai Founalei South (FS) in red, Mata Ua (MU) in blue, and Tahi Moana (TM) in yellow.

\section{DISCUSSION}

\section{Effects of de novo loci assembly parameter selection}

Consistently with previous analyses by Mastretta-Yanes et al. (2014) and Catchen et al. (2013b), we found that the core parameters for de novo loci assembly in STACKS -m, $-\mathrm{n}$, and -M, which limit the minimum number of identical raw reads required to create a stack of identical unique sequences for each individual, the maximum number of mismatches allowed between loci for each individual, and the maximum number of mismatches allowed between loci when building the catalog of all loci in a species, respectively, had the largest effects on the amount of loci and SNP generated from RAD sequence data. Although in this study we do not have access to a reference genome or sequencing replicates, with which parameter influences on de novo loci assembly error rates can be estimated, our analyses indicate that overall the population genetic inferences from datasets derived using a variety of assembly parameters were largely insensitive to parameter variations. This provides high confidence in the presented results, as the patterns of population genetic diversity here identified are most likely the result of true biological and ecological processes in the examined vent barnacle species, rather than methodological artifacts.

\section{No evidence supporting seamount-driven isolation in vent barnacle populations}

Altogether, we find no support for the seamount endemicity hypothesis in deep-sea hydrothermal-vent barnacles after examining thousands of genome-wide SNPs obtained from RAD-seq data. The patterns of genetic variation among individuals collected from seamount and spreading ridges, separated horizontally by hundreds of kilometers and vertically by hundreds of meters, did not reject the null hypothesis of panmixia within each species. Contrary to the predictions from the seamount endemicity hypothesis, we did not find higher population genetic structuring between seamount populations than between populations from spreading ridges.

Non-equilibrium processes, such as population expansion and recent colonization, can also lead to a lack of population structuring and apparently high gene flow. Evidence suggestive of non-equilibrium dynamics has been presented in populations of some of vent species, particularly in populations from fastspreading ridges (Vrijenhoek 2010). Commonly invoked process to explain negative Tajima's D and Fu's $F_{S}$ values and star-like haplotype networks in mitochondrial data, include bottlenecks and founder events resulting from processes such as catastrophic eruptions, vent formations and disappearances (Won et al. 
2003; Hurtado et al. 2004; Plouviez et al. 2009; Teixeira et al. 2011; Thaler et al. 2011; Beedessee et al. 2013; Thaler et al. 2014). Nonetheless, minor allele frequency spectra for vent barnacle populations in this study were consistent with mutation-drift equilibrium, as a majority of the alleles in each population have low frequencies (Luikart et al. 1998), with a long tail of alleles at intermediate frequencies approaching zero proportions. Therefore, we conclude that the lack of population structuring and high gene flow among examined vent barnacle populations does not seem to be caused by non-equilibrium processes, but rather by sufficient genetic exchange among among populations.

a)

Vulcanolepas scotiaensis
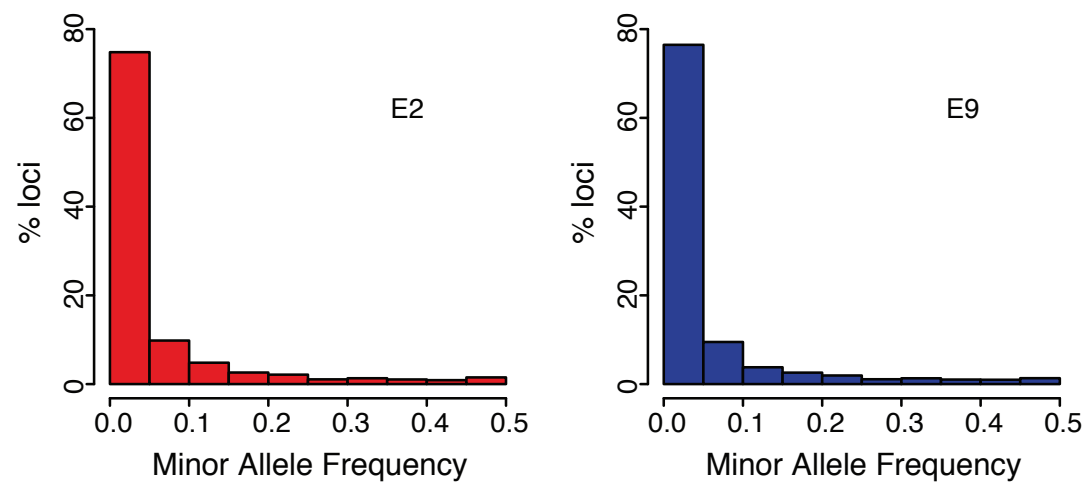

b)

Vulcanolepas osheai

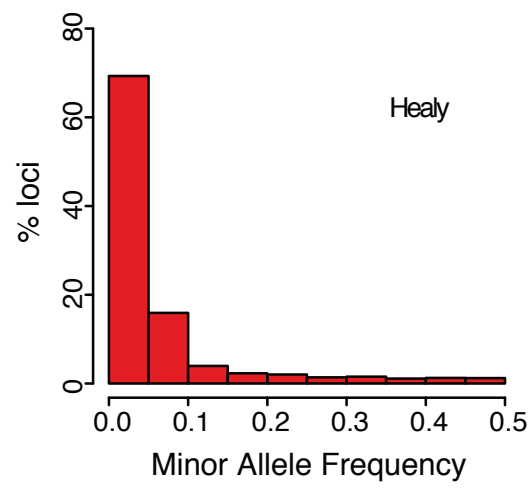

c)

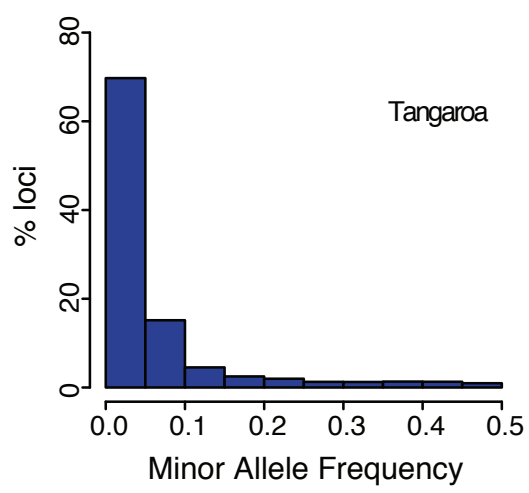

Eochionelasmus ohtai
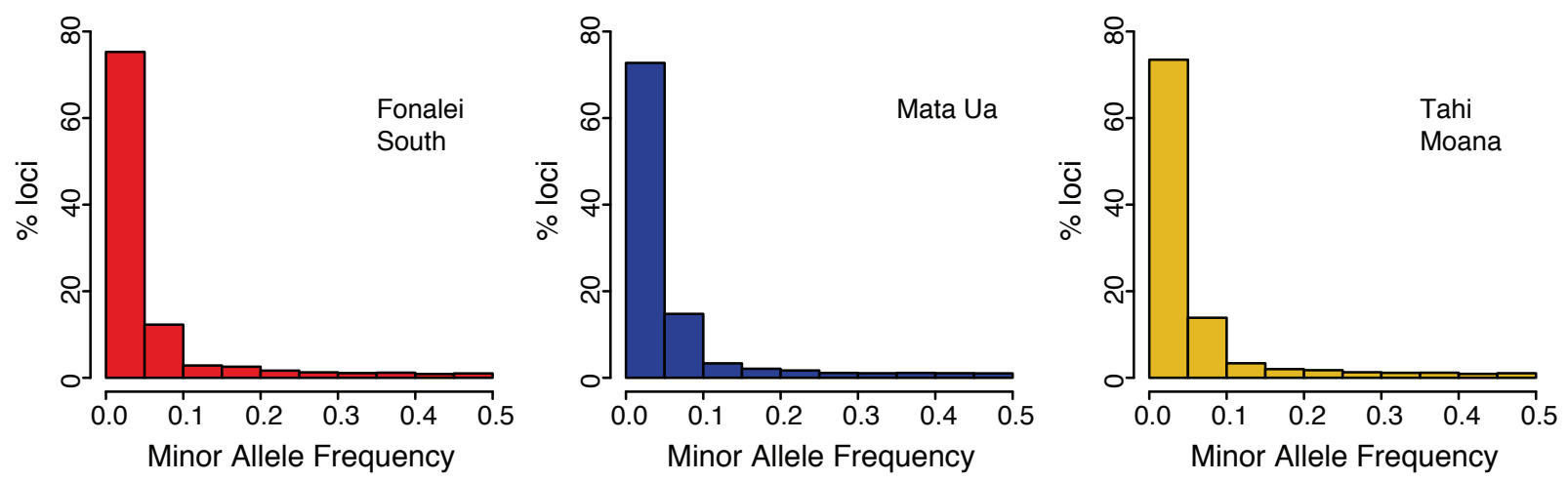
Figure 5. Minor allele frequency spectra from SNP loci for each vent barnacle population, per species. Labels and colors indicate the source population per species: a) V. scotiaensis - E2 in red and E9 in blue; b) V. osheai - Healy in red and Tangaroa in blue; c) E. ohtai - Founalei South in red, Mata Ua in blue, and Tahi Moana in yellow.

Larval development strategies have been long been hypothesized to be determinant of the dispersal potential of a species. Species in the vent barnacle clade A (sensu Herrera et al. (Chapter 3), to which $V$. osheai and V. scotiaensis belong, have large lecithotrophic naupliar larvae (Tunnicliffe \& Southward 2004; Watanabe et al. 2004). E. osheai is known to produce large eggs (Newman et al. 2006), and thus presumably also has lecithotrophic larvae. It is often assumed that the duration in the water column for lecithotrophic larvae is limited by yolk reserves and thus lecithotrophic larvae are expected to have a shorter development time than planktotrophic larvae, and therefore smaller dispersal potential overall. However, significant evidence has accumulated against this hypothesis. For example, results from a colonization study in the East Pacific Rise suggest that the large lecithotrophic larvae can successfully disperse over long distances exceeding 300 kilometers (Mullineaux et al. 2010) (the horizontal distances among barnacle populations in this study range between ca. 100 and 400 kilometers). Additionally, temperatures in the deep ocean are known to reduce metabolic and development rates and therefore enhance dispersal (O'connor et al. 2007). Large yolk reserves, combined with cold sea-bottom temperatures are known to yield pelagic larval durations of up to 100 days in species in the vent barnacle clade A (Yorisue et al. 2013). Taken together, these characteristics provide a potential mechanism for enabling dispersal over long distances in vent barnacles and may partially account for the absence of population genetic structuring.

\section{Local connectivity patterns in the East Scotia Ridge}

Consistent with our observations of genetic variability in $V$. scotianensis, recent population genetic studies of other endemic species from hydrothermal vents in the East Scotia Ridge (ESR) -namely the yeti crab Kiwa sp. and two gastropod species - show patterns of no population differentiation along the ridge, between the E2 and E9 sites (Roterman 2013). Interestingly, one of the gastropod species, the limpet Leptodrilus sp., also occurs in the neighboring Kemp caldera in of the South Sandwich Arc, a region from which $V$. scotiaensis is also known (Rogers et al. 2012). Roterman (2013) found significant differentiation between the ridge and Kemp caldera limpet populations, which is separated from the ridge axis by only approximately 96 kilometers to the east, whereas the E2 and E9 sites are separated from each other by approximately 440 kilometers. Nonetheless E2 and E9 sites are a located at similar depths of approximately 2,500 meters, whereas the Kemp caldera vents are approximately 1,000 meters shallower, which could explain the observed differentiation. Testing for population differentiation in $V$. scotiaensis 
between the ridge axis and the Kemp caldera would provide a good test for the generality of the observed depth isolation pattern in the limpet species.

Vulcanolepas scotiaensis
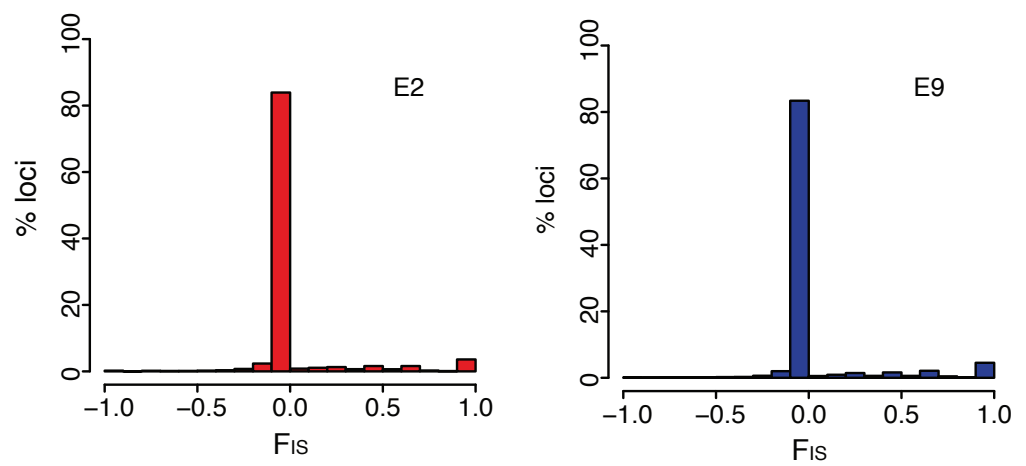

Vulcanolepas osheai
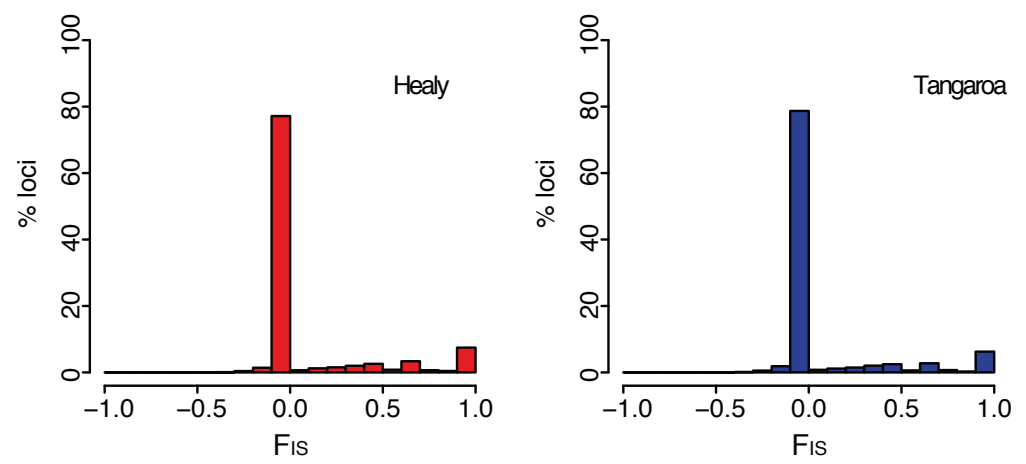

Eochionelasmus ohtai
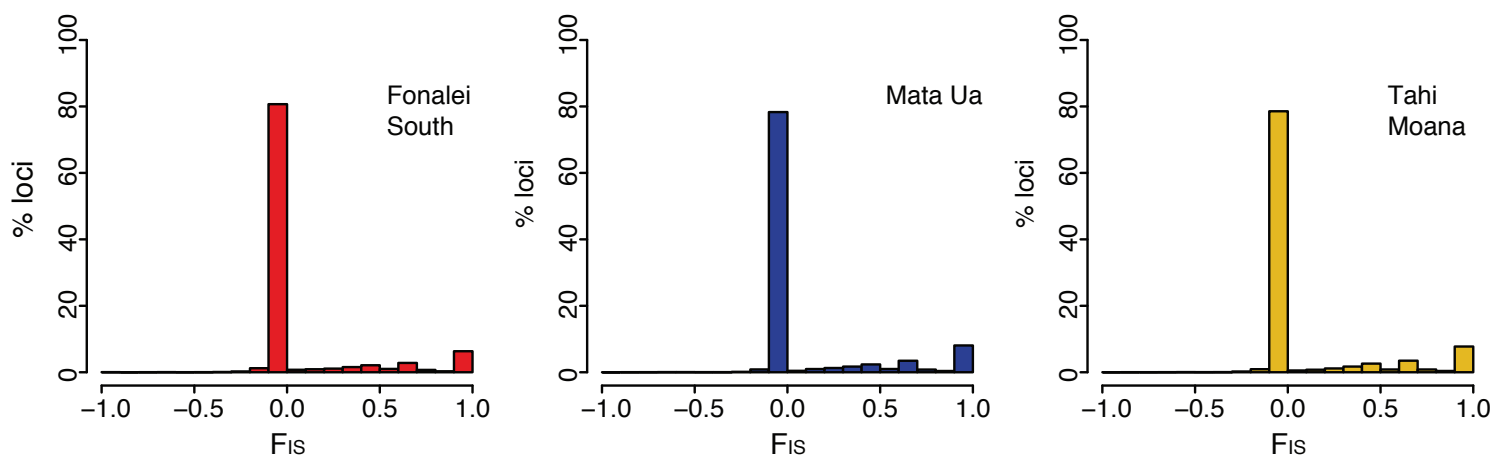

Figure 6. Frequency spectra of Wright's inbreeding index $\left(\mathrm{F}_{\mathrm{IS}}\right)$ values from SNP loci for each vent barnacle population, per species. Labels and colors indicate the source population per species: a) V. scotiaensis - E2 in red and E9 in blue; b) V. osheai - Healy in red and Tangaroa in blue; c) E. ohtai-Founalei South in red, Mata Ua in blue, and Tahi Moana in yellow.

Although mean water fluxes in the ESR have an easterly or north-easterly direction, largely influenced by the Antarctic Circumpolar Current (Meredith et al. 2008), the local hydrography and flows on the E2, E9 
and Kemp vent sites remain unknown. The ESR has an intermediate spreading rate of approximately 62 to 70 millimeters per year (Livermore 2003), which causes the formation of deep axial valleys with high walls that likely modify local current regimes. Mid-ocean ridges with similar characteristics are known to generate significant flows along and on the flanks of the ridge axes (Thurnherr et al. 2008; Thurnherr et al. 2011; Lavelle et al. 2012), thus potentially facilitating dispersal among spatially separated vent fields. Although hydrothermal vents at ridges with intermediate and low spreading rates - like the East Scotia Ridge - occur spatially less frequently along the ridge than vents at faster spreading systems - like the East Pacific Rise - (Baker \& German 2004), they tend to be significantly more stable over time, in some cases for thousands to hundreds of thousands of years (Lalou et al. 1995; Jamieson et al. 2013). Therefore, local vent formations and extinctions in these systems may not be recurrent enough to significantly influence genetic variability patterns in vent fauna. Finally, potential undiscovered vent sites along the East Scotia Ridge axis (German et al. 2000) could act as stepping-stones for vent barnacles and other vent species, and thus facilitate dispersal and gene-flow.

\section{Local connectivity patterns in the Kermadec arc}

The only previous population genetic study in seamount populations from the Kermadec arc (Smith et al. 2004) found heterogeneity in allozyme allelic frequencies and significant genetic differentiation between populations of Bathymodiolus vent mussels from two active seamounts of the Kermadec arc, Rumble V and Rumble III, which were separated horizontally by approximately $50 \mathrm{~km}$. However, this same study failed to reproduce the pattern of population structuring between seamount mussel populations when examining mitochondrial DNA sequences; therefore, it is possible that this pattern was an artifact of the allozyme markers. The use of other nuclear markers, such as SNP would clarify this discrepancy. Consistent with our results indicating high-gene flow among barnacle populations from Kermadec arc seamounts, Herrera et al. (Chapter 3) examined the phylogenetic relationships of vent barnacles and found no evidence of cryptic differentiation among $V$. oseahi individuals from Brothers, Clarck, Healy or Tangaroa seamounts.

Physical oceanographic processes could facilitate high non-directional dispersal and gene flow among seamounts in the southern Kermadec arc. Recent studies focusing on Brothers (Lavelle et al. 2008) and Rumble III seamounts (Stevens et al. 2014) indicate that circulation patterns in this region are highly dynamic over time, being largely influenced by isotropic tidal flows and mesoscale eddies, such as the East Cape Eddy. These studies indicate that flows speeds over these seamounts can range between 5 and 10 centimeters per second, which could potentially disperse larvae between 4 and 8 kilometers per day, and more than 130 to 260 kilometers in just a month. Futhermore, Stevens et al. (2014) found no evidence 
of Taylor column formation on the Rumble III seamount that could cause larval or particle retention around the summits.

The Kermadec arc is a region of high volcanic activity (de Ronde et al. 2007), which likely leads to catastrophic local extinctions, and frequent formation and disappearance of vents. Just between 2006 and 2012 there were at least three reported eruptions in active seamounts (Dodge 2009; Watts et al. 2012; Jutzeler et al. 2014). However, active seamounts usually host multiple vent fields, which are likely not affected by volcanic and venting dynamics simultaneously or at the same rates. We hypothesize that presence of multiple populations on other active neighboring seamounts in the southern Kermadec arc, such as Brothers, Rumble III, and Clark (A. Rowden, M. Clark, and D. Bowden personal communication), combined with potential high dispersal and gene-flow rates, may jointly act as buffers that prevent significant losses of genetic diversity due to vent instability. Samples from additional populations and further comparative studies with multiple taxa would allow us to elucidate more general patterns for vent populations in these seamounts.

\section{Local connectivity patterns in the Lau basin}

Consistent with our results from Lau basin populations, Plouviez et al. (2013) found no signatures of genetic differentiation or potential population structuring at horizontal scales from hundreds of meters up to 50 kilometers in Eochionelasmus ohtai from the Manus Basin, using mitochondrial markers. Although populations from the Manus basin have been considered a potentially different sub-species from the one inhabiting the Lau and North Fiji basins (Newman et al. 2006), a recent phylogenetic study by Herrera et al. (Chapter 3) found no evidence of genetic differentiation between Manus and Lau basin individuals of E. ohtai. Similar signatures of panmixia have been found in shrimp (Chorocaris sp. 2) and gastropod snail (Ifremeria nautilei) vent species within the Manus and Lau basins (Thaler et al. 2011; Thaler et al. 2014). Contrastingly Thaler et al. (2014) found significant structuring in populations of the squat lobster Munidopsis lauensis within Manus basin, which the authors attributed to potential larvae behaviors and water flow directionality.

In addition to the populations included in this study, E. ohtai is known from vents in other active systems in the Lau Basin, including the Eastern Lau Spreading Center and the North-East Lau Spreading Center (Herrera et al. Chapter 3), which can serve as dispersal stepping-stones for dispersal throughout the basin. The Lau basin has an extremely diverse and active geologic setting, with multiple isolated back-arc spreading ridges, axial volcanoes and active arc and back-arc seamounts hosting hydrothermal vent communities. Volcanic eruptions that create and destroy hydrothermal vent communities are also 
common in this region (Resing et al. 2011; Bohnenstiehl et al. 2014; Embley et al. 2014). However, as discussed earlier, we suggest that the presence of well-connected populations within a metapopulation in some vent species can act as buffers that prevent significant losses of genetic diversity due to vent instability. Ocean circulation at the scale of months in the Lau basin is dominated by isotropic flows caused by eddies, reaching speeds of up to 10 centimeters per second (Speer \& Thurnherr 2012), which could help explain the lack of directionality or differentiation observed in the genetic pool of E. otahi and other vent species within the basin.

\section{CONCLUSIONS}

Comparative data from three species of barnacles from deep-sea hydrothermal vents revealed patterns of genetic variation inconsistent with the seamount endemicity hypothesis. These results reinforce the idea that within-basin structuring is rare while between-basin structuring is common, lending insight into the scale at which vent populations are structured. Both the Kermadec Arc and Lau Basin are areas of high interest for polymetallic sulphide mining (Boschen et al. 2013). Although these results indicate that populations of vent barnacles in the examined populations could be resilient to potential disturbance from local mining restricted to focal sites, we suggest that further multi-species and time-series studies in these and other target and neighboring populations should take place in order to better assess the potential impacts of this extractive activity. This study constitutes the first use of genome-wide SNP data to examine patterns of population genetic structuring and connectivity among populations of vent species. We suggest that the seamount endemicity hypothesis warrants further testing using high-resolution SNP data in other vent organisms with differing life history strategies (e.g., brooders) that may limit their dispersal potential, as well as in non-vent organisms, which are not exposed to evolutionary pressures imposed by the dynamic nature of hydrothermal vent systems.

\section{ACKNOWLEDGEMENTS}

This research was supported by the Office of Ocean Exploration and Research of the National Oceanic and Atmospheric Administration (NA09OAR4320129 to TS); the Division of Ocean Sciences of the National Science Foundation (OCE-1131620 to TS); the Division of Polar Programs of the National Science Foundation (PLR-0739675 to TS); the Astrobiology Science and Technology for Exploring Planets program of the National Aeronautics and Space Administration (NNX09AB76G to TS); and the Academic Programs Office (Ocean Ventures Fund to SH), the Ocean Exploration Institute (Fellowship 
support to TMS) and the Ocean Life Institute of the Woods Hole Oceanographic Institution (internal grant to TMS and SH). For enabling access to key specimens we thank P. Tyler (NOCS), J. Copley (NOCS), A. Rogers (Oxford), N. Roterman (Oxford), M. Clark (NIWA), A. Rowden (NIWA), K. Schnabel (NIWA), S. Mills (NIWA), J. Resing (NOAA-PMEL), R. Embley (NOAA-PMEL), A. Reysenbach (PSU), M.K. Tivey (WHOI), the masters, crew, scientific personnel, and funding agencies of expeditions JC042, RR1211, TAN1104, TAN1206, and TN236. Specimens provided by the National Institute of Water and Atmospheric Research (NIWA) were collected under research programs: Ocean Survey 20/20 funded by

Land Information New Zealand; and the Impact of resource use on vulnerable deep-sea communities (CO1X0906), funded by MBIE. We also thank E. Bors, A. Tarrant, and A. Reitzel for providing helpful comments that improved this manuscript.

\section{REFERENCES}

Alberto F, Raimondi PT, Reed DC, et al. (2011) Isolation by oceanographic distance explains genetic structure for Macrocystis pyrifera in the Santa Barbara Channel. Molecular Ecology 20, 25432554.

Baco AR, Cairns SD (2012) Comparing molecular variation to morphological species designations in the deep-sea coral Narella reveals new Insights into seamount coral ranges. PLoS One 7, e45555.

Baird NA, Etter PD, Atwood TS, et al. (2008) Rapid SNP discovery and genetic mapping using sequenced RAD markers. PLoS One 3, e3376.

Baker ET, German CR (2004) On the Global Distribution of Hydrothermal Vent Fields. In: Mid-Ocean Ridges, pp. 245-266. American Geophysical Union.

Beedessee G, Watanabe H, Ogura T, et al. (2013) High connectivity of animal populations in deep-sea hydrothermal vent fields in the Central Indian Ridge relevant to its geological setting. PLoS One 8, e81570.

Beinart RA, Sanders JG, Faure B, et al. (2012) Evidence for the role of endosymbionts in regional-scale habitat partitioning by hydrothermal vent symbioses. Proceedings of the National Academy of Sciences of the United States of America 109, E3241-E3250.

Bohnenstiehl DR, Dziak RP, Matsumoto H, Conder JA (2014) Acoustic response of submarine volcanoes in the Tofua Arc and northern Lau Basin to two great earthquakes. Geophysical Journal International 196, 1657-1675.

Boschen RE, Rowden AA, Clark MR, Gardner JPA (2013) Mining of deep-sea seafloor massive sulfides: A review of the deposits, their benthic communities, impacts from mining, regulatory frameworks and management strategies. Ocean and Coastal Management 84, 54-67.

Catchen J, Bassham S, Wilson T, et al. (2013a) The population structure and recent colonization history of Oregon threespine stickleback determined using restriction-site associated DNA-sequencing. Molecular Ecology 22, 2864-2883.

Catchen J, Hohenlohe PA, Bassham S, Amores A, Cresko WA (2013b) Stacks: an analysis tool set for population genomics. Molecular Ecology 22, 3124-3140.

Cho W, Shank TM (2010) Incongruent patterns of genetic connectivity among four ophiuroid species with differing coral host specificity on North Atlantic seamounts. Marine Ecology 31, 121-143.

Christensen NL, Bartuska AM, Brown JH, et al. (1996) The report of the Ecological Society of America committee on the scientific basis for ecosystem management. Ecological Applications 6, 665-691. 
Clague GE, Jones WJ, Paduan JB, Clague DA, Vrijenhoek RC (2012) Phylogeography of Acesta clams from submarine seamounts and escarpments along the western margin of North America. Marine Ecology-an Evolutionary Perspective 33, 75-87.

D'Aloia CC, Bogdanowicz SM, Harrison RG, Buston PM (2014) Seascape continuity plays an important role in determining patterns of spatial genetic structure in a coral reef fish. Molecular Ecology 23, 2902-2913.

de Forges BR, Koslow JA, Poore GC (2000) Diversity and endemism of the benthic seamount fauna in the southwest Pacific. Nature 405, 944-947.

de Ronde CEJ, Baker ET, Massoth GJ, et al. (2007) Submarine hydrothermal activity along the midKermadec Arc, New Zealand: Large-scale effects on venting. Geochemistry, Geophysics, Geosystems 8, Q07007.

Dodge E (2009) Catastrophic volcanic activity at Rumble III volcano based on EM300 bathymetry and direct seafloor imaging, University of Washington.

Embley RW, Merle SG, Baker ET, et al. (2014) Eruptive modes and hiatus of volcanism at West Mata seamount, NE Lau basin: 1996-2012. Geochemistry, Geophysics, Geosystems 15, 4093-4115.

Emerson KJ, Merz CR, Catchen JM, et al. (2010) Resolving postglacial phylogeography using highthroughput sequencing. Proceedings of the National Academy of Sciences of the United States of America 107, 16196-16200.

German CR, Livermore RA, Baker ET, et al. (2000) Hydrothermal plumes above the East Scotia Ridge: an isolated high-latitude back-arc spreading centre. Earth and Planetary Science Letters 184, 241-250.

Herrera S, Reyes-Herrera PH, Shank TM (Chapter 2) Genome-wide predictability of restriction sites across the eukaryotic tree of life.

Herrera S, Shank TM (Chapter 5) RAD sequencing enables unprecedented phylogenetic resolution and objective species delimitation in recalcitrant divergent taxa.

Herrera S, Watanabe H, Shank T (Chapter 3) Evolutionary and biogeographical patterns of barnacles from deep-sea hydrothermal vents.

Hohenlohe PA, Bassham S, Etter PD, et al. (2010) Population genomics of parallel adaptation in threespine stickleback using sequenced RAD tags. PLoS Genetics 6, e1000862.

Hurtado LA, Lutz RA, Vrijenhoek RC (2004) Distinct patterns of genetic differentiation among annelids of eastern Pacific hydrothermal vents. Molecular Ecology 13, 2603-2615.

Jamieson JW, Hannington MD, Clague DA, et al. (2013) Sulfide geochronology along the Endeavour Segment of the Juan de Fuca Ridge. Geochemistry, Geophysics, Geosystems 14, 2084-2099.

Jutzeler M, Marsh R, Carey RJ, et al. (2014) On the fate of pumice rafts formed during the 2012 Havre submarine eruption. Nature Communications 5, Artn 3660.

Kelly RP, Palumbi SR (2010) Genetic structure among 50 species of the northeastern Pacific rocky intertidal community. PLoS One 5, e8594.

Lalou C, Reyss J-L, Brichet E, Rona PA, Thompson G (1995) Hydrothermal activity on a 105-year scale at a slow-spreading ridge, TAG hydrothermal field, Mid-Atlantic Ridge $26^{\circ} \mathrm{N}$. Journal of Geophysical Research: Solid Earth 100, 17855-17862.

Lavelle JW, Massoth GJ, Baker ET, de Ronde CEJ (2008) Ocean current and temperature time series at Brothers volcano. Journal of Geophysical Research-Oceans 113, C09018.

Lavelle JW, Thurnherr AM, Mullineaux LS, McGillicuddy DJ, Ledwell JR (2012) The prediction, verification, and significance of flank jets at mid-ocean ridges. Oceanography 25, 277-283.

Livermore R (2003) Back-arc spreading and mantle flow in the East Scotia Sea. Geological Society, London, Special Publications 219, 315-331.

Luikart G, Allendorf FW, Cornuet JM, Sherwin WB (1998) Distortion of allele frequency distributions provides a test for recent population bottlenecks. Journal of Heredity 89, 238-247.

Mastretta-Yanes A, Arrigo N, Alvarez N, et al. (2014) Restriction site-associated DNA sequencing, genotyping error estimation and de novo assembly optimization for population genetic inference. Molecular Ecology Resources 15, 28-41. 
Meredith MP, Garabato ACN, Gordon AL, Johnson GC (2008) Evolution of the deep and bottom waters of the Scotia Sea, Southern Ocean, during 1995-2005. Journal of Climate 21, 3327-3343.

Metaxas A (2011) Spatial patterns of larval abundance at hydrothermal vents on seamounts: evidence for recruitment limitation. Marine Ecology Progress Series 437, 103-117.

Mullineaux LS, Adams DK, Mills SW, Beaulieu SE (2010) Larvae from afar colonize deep-sea hydrothermal vents after a catastrophic eruption. Proceedings of the National Academy of Sciences of the United States of America 107, 7829-7834.

Mullineaux LS, Mills SW (1997) A test of the larval retention hypothesis in seamount-generated flows. Deep-Sea Research Part I-Oceanographic Research Papers 44, 745-\&.

Newman WA, Yamaguchi T, Southward AJ, Segonzac M (2006) Arthropoda, Crustacea, Cirripedia. In: Handbook of Deep-Sea Hydrothermal Vent Fauna (eds. Desbruyeres D, Segonzac M, Bright M), pp. 356-357. Denisia.

O'connor MI, Bruno JF, Gaines SD, et al. (2007) Temperature control of larval dispersal and the implications for marine ecology, evolution, and conservation. Proceedings of the National Academy of Sciences of the United States of America 104, 1266-1271.

Plouviez S, Schultz TF, McGinnis G, et al. (2013) Genetic diversity of hydrothermal-vent barnacles in Manus Basin. Deep-Sea Research Part I-Oceanographic Research Papers 82, 73-79.

Plouviez S, Shank TM, Faure B, et al. (2009) Comparative phylogeography among hydrothermal vent species along the East Pacific Rise reveals vicariant processes and population expansion in the South. Molecular Ecology 18, 3903-3917.

Prada C, Hellberg ME (2013) Long prereproductive selection and divergence by depth in a Caribbean candelabrum coral. Proceedings of the National Academy of Sciences of the United States of America 110, 3961-3966.

Reitzel AM, Herrera S, Layden MJ, Martindale MQ, Shank TM (2013) Going where traditional markers have not gone before: utility of and promise for RAD sequencing in marine invertebrate phylogeography and population genomics. Molecular Ecology 22, 2953-2970.

Resing JA, Rubin KH, Embley RW, et al. (2011) Active submarine eruption of boninite in the northeastern Lau Basin. Nature Geoscience 4, 799-806.

Rogers AD, Morley S, Fitzcharles E, Jarvis K, Belchier M (2006) Genetic structure of Patagonian toothfish (Dissostichus eleginoides) populations on the Patagonian Shelf and Atlantic and western Indian Ocean Sectors of the Southern Ocean. Marine Biology 149, 915-924.

Rogers AD, Tyler PA, Connelly DP, et al. (2012) The discovery of new deep-sea hydrothermal vent communities in the southern ocean and implications for biogeography. PLoS Biology 10, e1001234.

Roterman CN (2013) The Evolution and Population Genetics of Hydrothermal Vent Megafauna from the Scotia Sea, University of Oxford.

Ryan WBF, Carbotte SM, Coplan JO, et al. (2009) Global multi-resolution topography synthesis. Geochemistry, Geophysics, Geosystems 10, Q03014.

Smith PJ, McVeagh SM, Won Y, Vrijenhoek RC (2004) Genetic heterogeneity among New Zealand species of hydrothermal vent mussels (Mytilidae : Bathymodiolus). Marine Biology 144, 537-545.

Speer K, Thurnherr AM (2012) The Lau Basin float experiment (LAUB-FLEX). Oceanography 25, 284285.

Stevens CL, Consalvey M, Devine JA, Clark MR (2014) Mixing and transport near the shallow-crested Rumble III seamount and the implications for plankton distribution. New Zealand Journal of Marine and Freshwater Research 48, 194-215.

Teixeira S, Cambon-Bonavita MA, Serrao EA, Desbruyeres D, Arnaud-Haond S (2011) Recent population expansion and connectivity in the hydrothermal shrimp Rimicaris exoculata along the Mid-Atlantic Ridge. Journal of Biogeography 38, 564-574.

Thaler AD, Plouviez S, Saleu W, et al. (2014) Comparative population structure of two deep-sea hydrothermal-vent-associated decapods (Chorocaris sp 2 and Munidopsis lauensis) from southwestern Pacific back-arc basins. PLoS One 9, e101345. 
Thaler AD, Zelnio K, Saleu W, et al. (2011) The spatial scale of genetic subdivision in populations of Ifremeria nautilei, a hydrothermal-vent gastropod from the southwest Pacific. BMC Evolutionary Biology 11, 372.

Thoma J, Pante E, Brugler M, France S (2009) Deep-sea octocorals and antipatharians show no evidence of seamount-scale endemism in the NW Atlantic. Marine Ecology Progress Series 397, 25-35.

Thurnherr AM, Ledwell JR, Lavelle JW, Mullineaux LS (2011) Hydrography and circulation near the crest of the East Pacific Rise between 90 degrees and 10 degrees N. Deep-Sea Research Part IOceanographic Research Papers 58, 365-376.

Thurnherr AM, Reverdin G, Bouruet-Aubertot P, et al. (2008) Hydrography and flow in the Lucky Strike segment of the Mid-Atlantic Ridge. Journal of Marine Research 66, 347-372.

Tunnicliffe V, Koop BF, Tyler J, So S (2010) Flatfish at seamount hydrothermal vents show strong genetic divergence between volcanic arcs. Marine Ecology-an Evolutionary Perspective 31, 158167.

Tunnicliffe V, Southward AJ (2004) Growth and breeding of a primitive stalked barnacle Leucolepas longa (Cirripedia : Scalpellomorpha : Eolepadidae : Neolepadinae) inhabiting a volcanic seamount off Papua New Guinea. Journal of the Marine Biological Association of the United Kingdom 84, 121-132.

Van Dover CL (2000) The Ecology of Deep-Sea Hydrothermal Vents Princeton University Press, Princeton, N.J.

Van Dover CL (2010) Mining seafloor massive sulphides and biodiversity: what is at risk? ICES Journal of Marine Science 68, 341-348.

Vrijenhoek RC (2010) Genetic diversity and connectivity of deep-sea hydrothermal vent metapopulations. Molecular Ecology 19, 4391-4411.

Watanabe H, Kado R, Tsuchida S, et al. (2004) Larval development and intermoult period of the hydrothermal vent barnacle Neoverruca sp. Journal of the Marine Biological Association of the United Kingdom 84, 743-745.

Watanabe H, Tsuchida S, Fujikura K, et al. (2005) Population history associated with hydrothermal vent activity inferred from genetic structure of neoverrucid barnacles around Japan. Marine EcologyProgress Series 288, 233-240.

Watts AB, Peirce C, Grevemeyer I, et al. (2012) Rapid rates of growth and collapse of Monowai submarine volcano in the Kermadec Arc. Nature Geoscience 5, 510-515.

White C, Selkoe KA, Watson J, et al. (2010) Ocean currents help explain population genetic structure. Proceedings of the Royal Society B-Biological Sciences 277, 1685-1694.

Wilson RR, Kaufmann RS (1986) Seamount biota and biogeography. In: Seamounts, Islands, and Atolls (eds. Keating BH, Fryer P, Batiza R, Boehlert GW), Washington DC.

Won Y, Young CR, Lutz RA, Vrijenhoek RC (2003) Dispersal barriers and isolation among deep-sea mussel populations (Mytilidae : Bathymodiolus) from eastern Pacific hydrothermal vents. Molecular Ecology 12, 169-184.

Yorisue T, Kado R, Watanabe H, et al. (2013) Influence of water temperature on the larval development of Neoverruca sp and Ashinkailepas seepiophila-Implications for larval dispersal and settlement in the vent and seep environments. Deep-Sea Research Part I-Oceanographic Research Papers 71, 33-37.

\section{SUPPORTING INFORMATION}




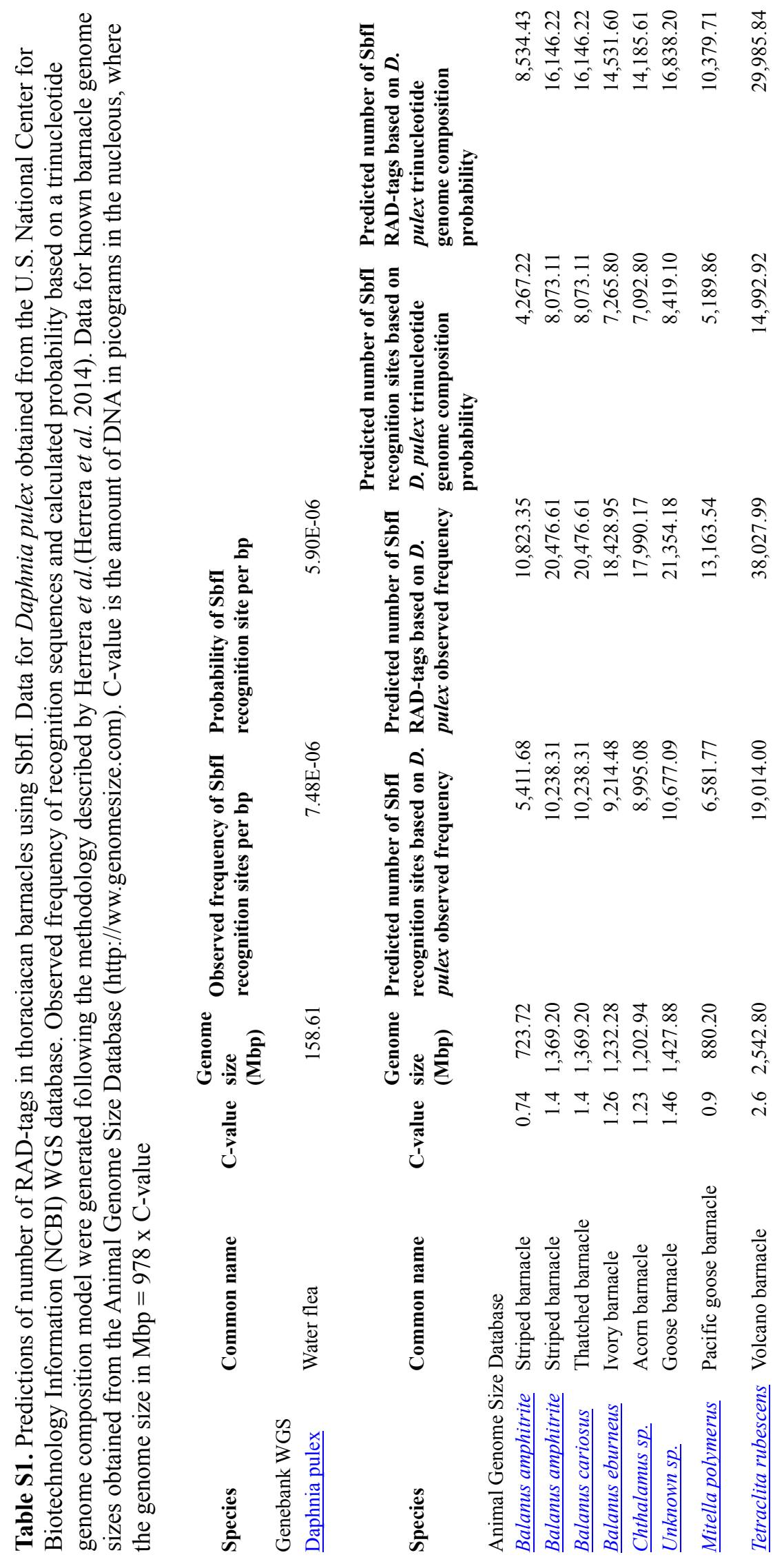




\title{
CHAPTER 5
}

\section{RAD sequencing enables unprecedented phylogenetic resolution and objective species delimitation in recalcitrant divergent taxa}

\begin{abstract}
Species delimitation is problematic in many taxa due to the difficulty of evaluating predictions from species delimitation hypotheses, which chiefly relay on subjective interpretations of morphological observations and/or DNA sequence data. This problem is exacerbated in recalcitrant taxa for which genetic resources are scarce and inadequate to resolve questions regarding evolutionary relationships and uniqueness. In this case study we demonstrate the empirical utility of restriction site associated DNA sequencing (RAD-seq) by unambiguously resolving phylogenetic relationships among recalcitrant octocoral taxa with divergences greater than 80 million years. We objectively infer robust species boundaries in the genus Paragorgia, which contains some of the most important ecosystem engineers in the deep-sea, by testing alternative taxonomy-guided or unguided species delimitation hypotheses using the Bayes factors delimitation method (BFD*) with genome-wide single nucleotide polymorphism data. We present conclusive evidence rejecting the current morphological species delimitation model for the genus Paragorgia and indicating the presence of cryptic species boundaries associated with environmental variables. We argue that the suitability limits of RAD-seq for phylogenetic inferences in divergent taxa cannot be assessed in terms of absolute time, but depend on taxon-specific factors such as mutation rate, generation time and effective population size. We show that classic morphological taxonomy can greatly benefit from integrative approaches that provide objective tests to species delimitation hypothesis. Our results pave the way for addressing further questions in biogeography, species ranges, community ecology, population dynamics, conservation, and evolution in octocorals and other marine taxa.
\end{abstract}




\section{INTRODUCTION}

Species delimitation is problematic in many taxa due to the difficulty of evaluating predictions from species delimitation hypotheses derived using different species concepts. Species concepts set particular expectations of the properties used to support species delimitations (De Queiroz 2007). For example, the classic biological species concept requires intrinsic reproductive isolation between heterospecific organisms and interbreeding among homospecific organisms resulting in viable and fertile descendants (Mayr 1942; Dobzhansky 1970). In many cases, if not the majority, it is difficult to evaluate behavioral, reproductive, and ecological properties due to technical limitations of field or laboratory work, which largely determine the kind of observations and data that can be obtained. In these cases researchers conventionally rely on morphological observations and/or DNA sequence data to generate species delimitation hypotheses.

Although there have been significant attempts at developing statistical methods to objectively identify species-diagnostic morphological discontinuities (e.g., Zapata \& Jimenez 2012), most species delimitations continue to be performed subjectively based on assessments made by specialized taxonomists. Molecular phylogenetic analyses of DNA sequences provide an independent way to test these species delimitation hypotheses utilizing a variety of methods, ranging from variability thresholds of barcode sequences (Hebert et al. 2003), to probabilistic coalescent-based model methods (Pons et al. 2006; Yang \& Rannala 2010; Fujisawa \& Barraclough 2013; Grummer et al. 2014). These molecular methods rely on informative DNA sequence markers, and in many cases on resolved phylogenies.

The sub-class Octocorallia (Phylum Cnidaria), which includes animals known as gorgonians, sea pens, and soft corals, is an example of a recalcitrant group where species delimitations are problematic. Octocorals are predominantly a deep-sea group (Cairns 2007; Roberts \& Cairns 2014) and therefore are extremely difficult to observe and collect. Classic morphology-based species delimitation and identification in this group is arduous for non-specialists, and challenging to replicate among taxonomists (Daly et al. 2007; McFadden et al. 2010b). Variations in octocoral colony architecture and micro-skeletal structures - sclerites - are used as species diagnostic characters (Bayer 1956). However, studies over the last 15 years have shown that in many cases species delimitations and systematics based on these morphological traits keep little to no correspondence with the patterns of genetic diversity and relatedness inferred using mitochondrial and ribosomal DNA sequence markers (McFadden et al. 2006; Clark et al. 2007; France 2007; Dueñas \& Sánchez 2009). A confounding factor when analyzing mitochondrial DNA markers is the fact that anthozoans, including octocorals, have slow rates of sequence evolution relative to 
other metazoans (Shearer et al. 2002; Hellberg 2006). Furthermore, octocoral mitochondrion is unique among eukaryotes by having a functional DNA mismatch repair gene - mtMutS - which presumably is responsible for the extremely low sequence variability observed in this group (Bilewitch \& Degnan 2011). Traditional molecular markers have thus been remarkably insufficient to resolve relationships at all taxonomic levels within the octocorals (Berntson et al. 2001; France et al. 2002; Mcfadden et al. 2004; Smith et al. 2004; Thoma et al. 2009; Dueñas et al. 2014). Alternative nuclear markers, such as the ITS2 and SRP54 have been used to examine interspecific and intraspecific relationships (Aguilar \& Sánchez 2007; Concepcion et al. 2007; Grajales et al. 2007; Herrera et al. 2010); however, their application and impact has been limited due to issues regarding intragenomic variability (Sanchez \& Dorado 2008) and low sequencing reliability (Mcfadden et al. 2010a). These long-standing technical problems have caused fundamental questions in octocorals regarding species differentiation, systematics, diversity, biogeography, and species ranges to remain unanswered.

Technological developments in next-generation sequencing platforms and library preparation methodologies have made genomic resources increasingly accessible and available for the study of nonmodel organisms, thus offering a great opportunity to overcome the difficulties inherent to the use of traditional sequencing approaches. One of these methodologies is restriction-site-associated DNA sequencing (RAD-seq), which combines enzymatic fragmentation of genomic DNA with high-throughput sequencing for the generation of large numbers of markers (Baird et al. 2008). RAD-seq has shown great promise to resolve difficult phylogenetic, phylogeographic, and species delimitation questions in diverse groups of eukaryotes (Emerson et al. 2010; Nadeau et al. 2012; Wagner et al. 2012; Eaton \& Ree 2013; Jones et al. 2013; Cruaud et al. 2014; Escudero et al. 2014; Hipp et al. 2014; Leache et al. 2014; Herrera et al. Chapter 3), including cnidarians (Reitzel et al. 2013) and most recently deep-sea octocorals (Pante et al. 2014). The number of orthologous restriction sites that can be retained across taxa, which decreases as divergence increases, limits the usefulness of RAD-seq for these kinds of studies. In silico studies in model organisms indicate that RAD-seq can be used to infer phylogenetic relationships in young groups of species (up to 60 million years old), such as Drosophila (Rubin et al. 2012; Cariou et al. 2013; Seetharam \& Stuart 2013); however, the real limits of this technique have not been significantly explored.

In this study we aim to empirically explore the limits of RAD-seq to solve questions in phylogenetics and species delimitation. We focus on the recalcitrant Anthomastus-Corallium clade of octocorals (sensu McFadden et al. 2006) to test the utility of RAD-seq to resolve phylogenetic relationships among divergent taxa, and to infer objective species boundaries. Corals in the Anthomastus-Corallium clade (hereafter referred as the AC clade) are among the most conspicuous, widely distributed, and ecologically 
important benthic invertebrates in deep-water ecosystems (Roberts et al. 2009; Wating et al. 2011). This clade is constituted by more than 100 species defined morphologically, divided in 10 genera, and three families (World Register of Marine Species at http://www.marinespecies.org accessed on 2014-10-10), spanning a divergence time of over 100 million years (Ardila et al. 2012; Herrera et al. 2012). However, species delimitations and phylogenetic relationships in this clade, as in other octocorals, are controversial and conflictive (Herrera et al. 2010; Ardila et al. 2012; Herrera et al. 2012). Many of the species in this group are considered species indicators of Vulnerable Marine Ecosystems (e.g. ICES 2013), with some of them considered endangered (CITES 2014). Accurate species identifications, as well as complete inventories and knowledge of species ranges, are therefore critical to ensure the effectiveness and appropriateness of conservation and management policies.

\section{RESULTS}

\section{Morphological species identifications}

Using current species descriptions, colony observations, and scanning electron microscopy of sclerites, we identified a total of 12 putative morphological species among the 44 examined specimens from the AC clade (Table S1). These species correspond to the genera Paragorgia (P. arborea, P. stephencairnsi, $P$. johnsoni, P. maunga, P. alisonae, P. kaupeka, and P. coralloides) and Sibogagorgia (S. cauliflora) of the family Paragorgiidae; Hemicorallium (H. laauense-imperiale) and Corallium of the family Coralliidae; and Anthomastus and Heteropolypus of the family Alcyoniidae.

\section{Octocorals are amenable to RAD sequencing}

We generated a dense genome-wide set of genetic markers from the 44 AC clade specimens via RAD sequencing, using the 6-cutter restriction enzyme PstI, and used them to perform phylogenetic inferences and species delimitation analyses. We obtained roughly $3.9 \pm 1.4$ million reads (average \pm standard deviation) per individual, of which $74.3 \pm 8.1 \%$ were retained after stringent quality filtering steps (Table $\mathrm{S} 2)$.

\section{Optimization of RAD-loci clustering parameters}

To examine the sensitivity of the phylogenetic inference to the clustering parameters used to identify loci and create nucleotide matrices in the program pyRAD (Eaton 2014), we investigated different combinations of clustering thresholds (c $0.80,0.85$ and 0.90) and minimum number of taxa per locus (m 4, 6, and 9) in a reduced 'backbone' matrix (hereafter matrix names will be highlighted in bold) containing one individual from each of the 12 morphological species. The 9 resulting backbone matrices 
ranged in the total number of loci per matrix from approximately 9 to 60 thousand loci, increasing dramatically as the minimum number of taxa per locus was reduced (Table S3). In contrast, the different clustering thresholds did not have a significant effect on the total number of loci, but rather on the number of variable sites and, most importantly, on the number of phylogenetically informative sites (Table S3). Each resulting backbone matrix analyzed in RAxML (Stamatakis 2006) produced identical stronglysupported tree topologies (Fig. S1). We selected c 0.80 (80\% similarity among sequences) and $\mathrm{m} 9$ (minimum coverage of taxa per locus of $75 \%$ ) as the optimal combination of loci-clustering parameters because they minimized the proportion of missing data (0.20) in the matrix while maximizing the fraction of variable sites that were phylogenetically informative (0.24) (Table S3). The proportion of shared loci among individuals of Paragorgiidae and Coralliidae, lineages whose split has been estimated to be between 80-150 million years ago (Ardila et al. 2012; Herrera et al. 2012), was remarkably high (70$80 \%$ ) (Fig. 1).

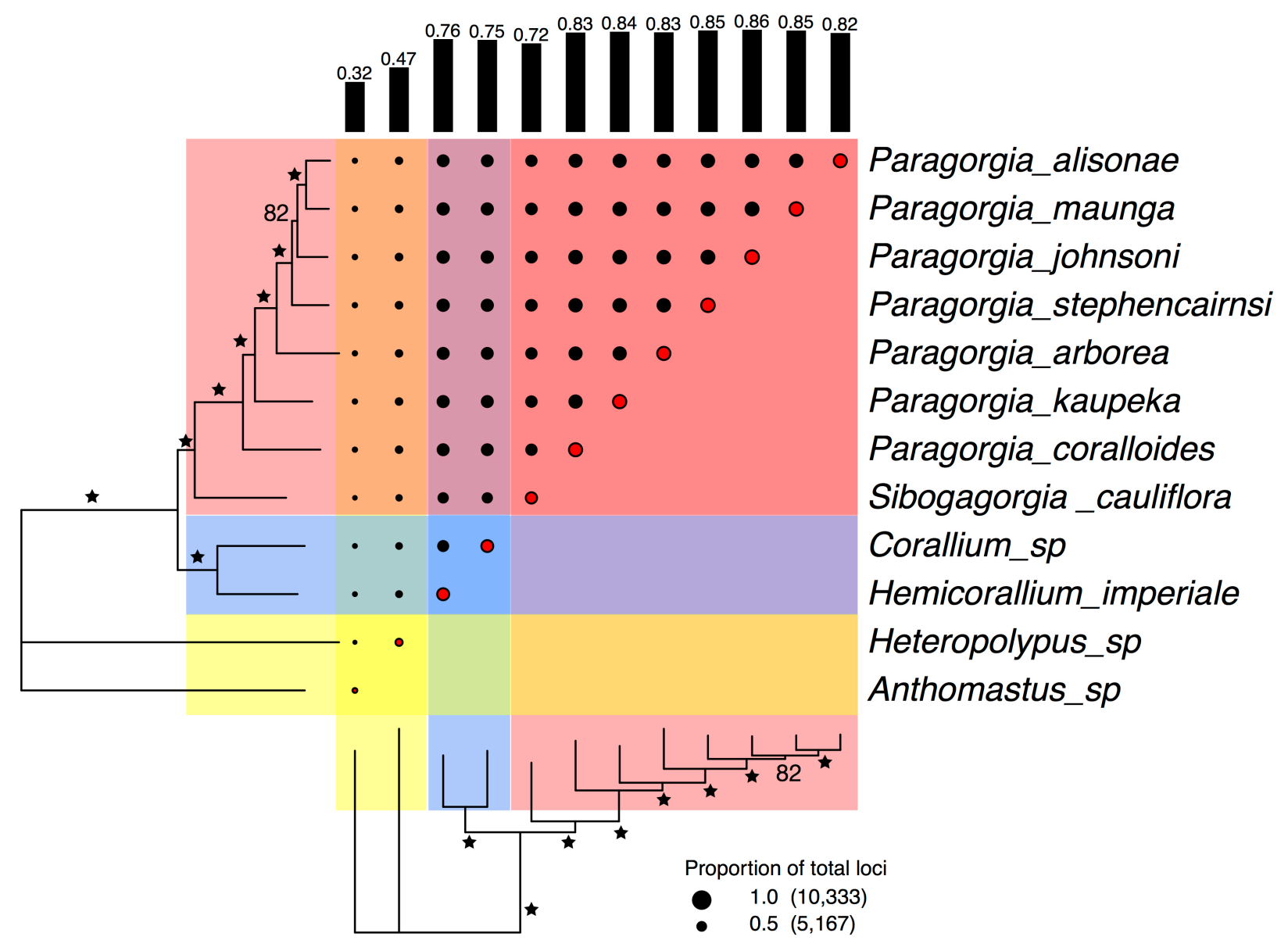

Figure 1. Proportion of loci shared among individuals of the AC clade in the optimal backbone matrix (c $0.80, \mathrm{~m}$ 9). Each family is indicated with a different color: red for Paragorgiidae; blue for Coralliidae; and yellow for 
Alcyoniidae. Black-filled circles represent the proportion of the total number of loci shared among individuals. Redfilled circles represent the proportion of the total number of loci present in each individual. Circle scale shows the number of loci represented by 1.0 and 0.5 circle sizes. Black vertical bars represent the average proportion of loci shared by each individual. Phylogenetic tree was inferred with RAxML. Stars on the tree represent branch bootstrap support of 100. Smaller bootstrap support values are indicated with numbers. This figure was generated with the package RADami (Hipp et al. 2014).

We used the selected optimal loci-clustering parameters to generate the 'PHYLO' matrix, containing the sequence data of all the 44 octocoral specimens. The use of the parameter value c 0.80 yielded approximately $71 \pm 15$ thousand loci - with a minimum depth of coverage of $5 \mathrm{x}$ and after filtering for paralogs- per specimen (mean depth of clusters used in loci construction was $23 \pm 8 x$ ) (Table S4). The PHYLO matrix contained a total of 5,997 loci that contained data for at least $75 \%$ of the specimens (after a second paralog removal). There were 85,293 variable sites in this matrix, of which 53,150 were phylogenetically informative.

\section{RAD-seq data support a fully resolved phylogeny}

The phylogenetic analysis of the PHYLO concatenated RAD-seq matrix produced a completely resolved evolutionary tree of the AC specimens (Fig. 2). In general, all branches were supported by high (greater than 95) bootstrap values, except for the one supporting the clade of P. johnsoni, P. alisonae, and $P$. maunga. Each one of the morphologically identified families, genera, and species in this dataset were monophyletic. The branching pattern of the tree is consistent with an expected transition between coalescent processes among species and genera (long deep branches), and population processes within species (short shallow branches).

The topology of the tree obtained with a traditional 'mitochondrial' matrix (711 base pairs of the mtMutS gene containing 130 variable sites, of which 101 were phylogenetically informative) was incongruent with the PHYLO tree (Fig. 2). The mitochondrial tree indicated a well-supported alternative divergence order for P. coralloides and P. kaupeka in the Paragorgia clade. In addition, the families Paragorgiidae (bubblegum corals) and Coralliidae (precious corals) were not monophyletic. The bubblegum coral genus Sibogagorgia appeared more closely related to the precious corals than to the other bubblegum coral genus Paragorgia, and the genera Corallium and Hemicorallium did not form a clade. However, these alternative relationships were not significantly supported by the bootstrap analysis,. Indeed, a substantial proportion of branches on the mitochondrial tree were poorly supported (bootstrap values smaller than $80 \%)$. 


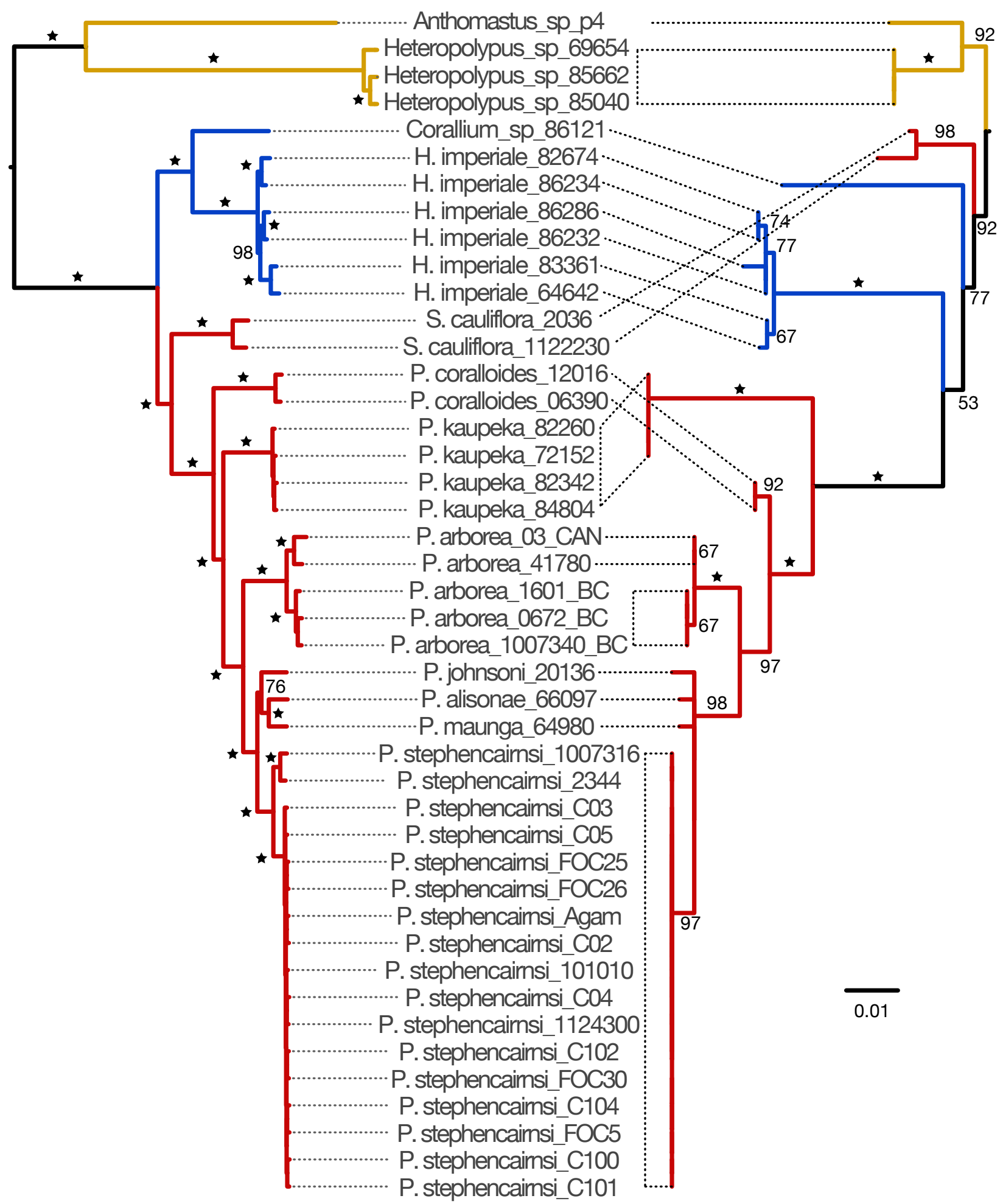

Figure 2. Phylogenetic trees of the $\mathrm{AC}$ clade based on RAD-seq and mitochondrial data. Left tree based on the RAD-seq concatenated PHYLO matrix. Right tree based on the mtMutS mitochondrial matrix. Each family is indicated with a different branch color: blue red for Paragorgiidaea; blue for Coralliidae; and yellow for 
Alcyoniidae. Phylogenetic trees were inferred with RAxML. Stars on the trees represent bootstrap support of 100. Smaller bootstrap values are indicated in numbers. Scale bar indicates substitutions per site.

\section{RAD-seq data reveal cryptic genetic diversity}

Branch-length differences among individuals, as well as well-supported sub-clades, revealed intraspecific genetic diversity that was undetected by the mitochondrial matrix. Two sub-clades were revealed by the phylogenetic analysis of the PHYLO matrix in the P. arborea and P. stephencairnsi clades. The subclades in P. arborea correspond to a pattern of segregation by geographic location with specimens from the north Pacific in one sub-clade, and specimens from the south Pacific and north Atlantic in the other. Contrastingly, the sub-clades in P. stephencairnsi correspond to a pattern of segregation by depth with specimens collected shallower than $350 \mathrm{~m}$ in one sub-clade, and specimens collected deeper than $1000 \mathrm{~m}$ in the other.

\section{Current morphological species delimitation is rejected}

To evaluate the utility of RAD-seq to perform objective species delimitations in octocorals we focused on specimens the genus Paragorgia as it was the best-sampled taxon in our dataset, both in terms of geographic representation and number of morphological species. We used the Bayes Factor Delimitation method with genomic data (BFD*) (Leache et al. 2014), which allows for the comparison of conflictive species delimitation models in an explicit multispecies coalescent framework using genome-wide single nucleotide polymorphism (SNP) data. We calculated marginal likelihoods of taxonomy-guided and taxonomy-unguided species delimitation models from a matrix of unlinked SNPs including only specimens of Paragorgia ('PARAGORGIA' matrix containing 1,203 SNPs present in all individuals). We compared the marginal likelihood estimates of alternative species delimitation models to the null model 'morphid', which is based on current morphological species descriptions, using Bayesian factors.

The null model, morphid, was rejected in favor of alternative species delimitation models for Paragorgia (Fig. 3) (morphid was ranked $7^{\text {th }}$ among 10 evaluated models in terms of the marginal likelihood estimate). The 'PABSTE' model, which proposes 9 species based on the 7 morphological species in the dataset plus splits corresponding to the sub-clades in P. arborea and in P. stephencairnsi, received decisive support from Bayes factors as the best species delimitation model. The taxonomy-unguided model 'geo' - which splits the specimens based on the geographic location where they were collected and the models proposed by the Poisson tree processes (PTP) method based on the mitochondrial data matrix, were the lowest ranked and most strongly rejected models overall. 


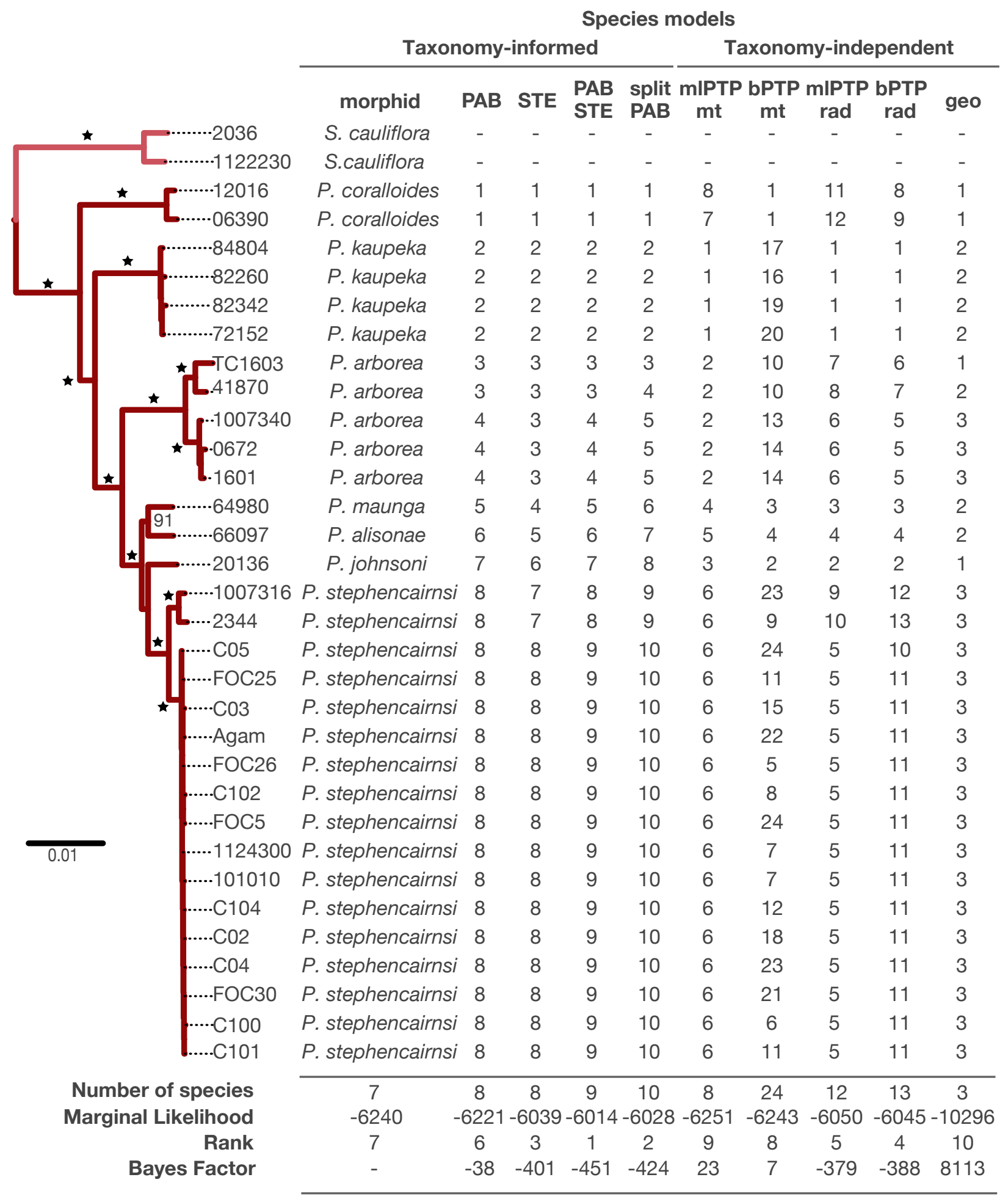

Figure 3. Species delineation hypotheses for Paragorgia. Table shows the different species delimitation models for Paragorgia evaluated with the BFD* method and their results. Sibogagorgia was included as outgroup to root the inferences for Paragorgia. Each row indicates a different specimen. Each column indicates a different species 
delimitation model. The first column, model morphid, indicates the species identifications based on morphology. For all other models, numbers indicate the species assignments. Bottom rows show the total number of species proposed, the marginal likelihood estimate, and rank for each model. The Bayes factor comparisons were calculated with respect to the null morphid model. Phylogenetic tree on the left, shown only for visual reference, was inferred with the RAD-seq concatenated PARAGORGIID matrix in RAxML. Each genus is indicated with a different branch color: pink for Sibogagorgia; and dark red for Paragorgia. Stars on the trees represent bootstrap support of 100. Smaller bootstrap values are indicated in numbers. Scale bar indicates substitutions per site.

\section{Concatenated and coalescent species tree analyses are congruent}

The topology of the species tree inferred using the SNP PARAGORGIA matrix was entirely congruent with the topology generated by the maximum likelihood phylogenetic analysis of the concatenated sequence matrices (Fig. 4). The species tree analysis also greatly improved support for the clade of $P$. johnsoni, P. alisonae, and P. maunga. The posterior distribution of species trees indicated a small fraction of conflictive topologies concentrating in this region of the tree.

\section{DISCUSSION}

\section{RAD sequencing enables unprecedented phylogenetic resolution}

Our analyses of RAD-seq data provide a robust phylogenetic hypothesis for the recalcitrant octocorals in the Anthomastus-Corallium clade, a result never achieved before. Moreover, this study, together with the work by Pante et al. (2014) in the octocoral genus Chrysogorgia, constitute the first applications of RADsequencing for phylogenetics and species delimitation in cnidarians. Only a handful of previous studies, using traditional mitochondrial data and the ITS2 and 28S nuclear markers, have attempted to evaluate phylogenetic relationships in the octocoral AC clade (Herrera et al. 2010; Ardila et al. 2012; Brockman \& McFadden 2012; Herrera et al. 2012; McFadden \& van Ofwegen 2013; Uda et al. 2013; Figueroa \& Baco 2014). These studies find support for the monophyly of the genus Paragorgia, the family Coralliidae, and the sister relationship between the Paragorgiidae and Coralliidae. However, those data do not provide enough phylogenetic resolution to infer the evolutionary relationships among many of the putative morphological species. Furthermore, significant incongruences between mitochondrial and nuclear ITS2 gene trees from AC taxa have been documented (Herrera et al. 2010). Here we reproduce similar incongruences when comparing the trees inferred from mitochondrial and RAD-seq datasets (Fig. 2). Likewise, Pante et al. (2014) documented marked incongruence between trees inferred from mitochondrial and RAD-seq data in Chyrsogorgia. These observations suggest that processes that can 
cause gene tree heterogeneity, such as incomplete lineage sorting and horizontal gene transfer (Maddison 1997; Edwards 2009), may be more prevalent in octocorals than previously recognized.

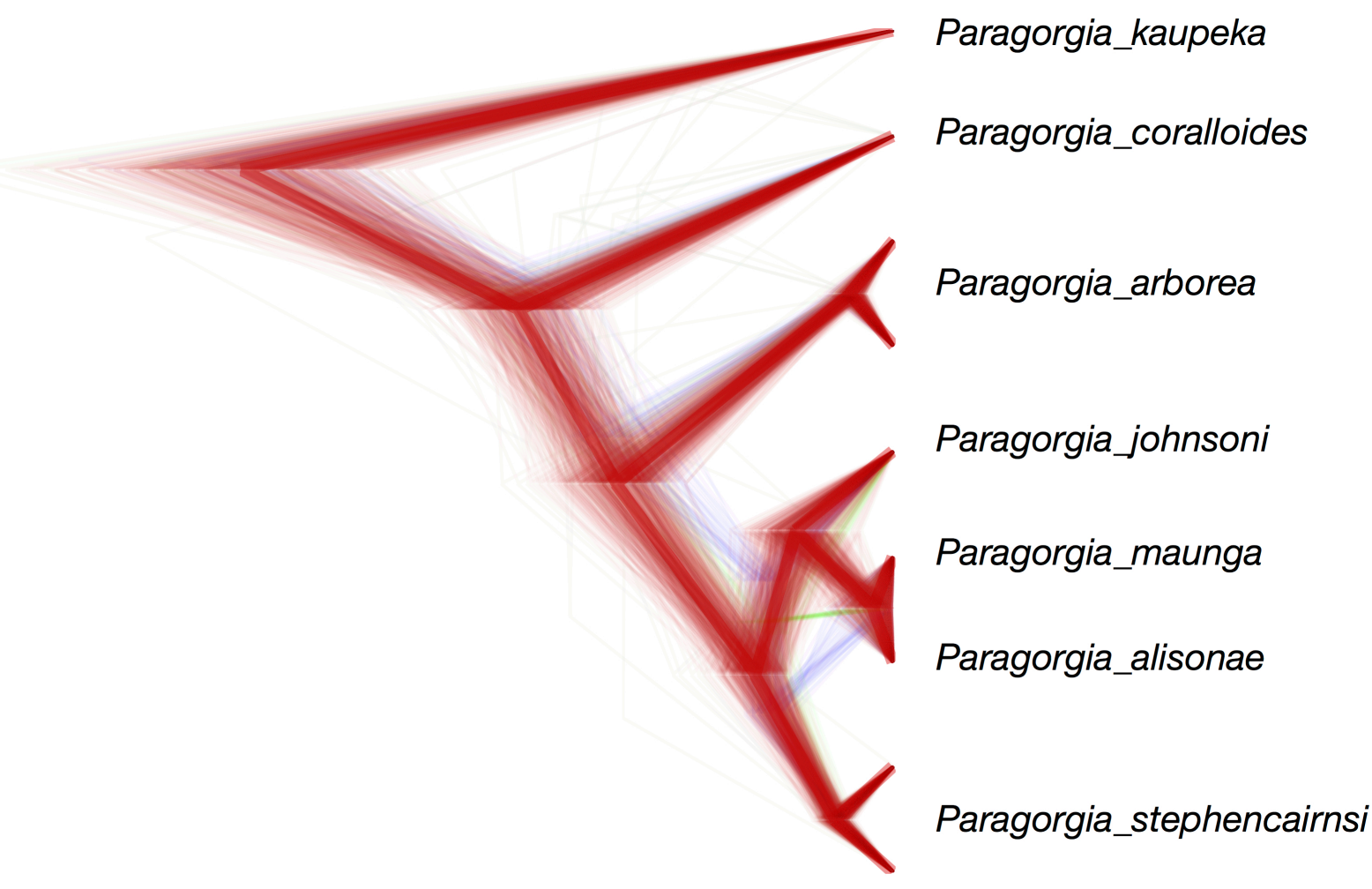

Figure 4. Species tree of Paragorgia. This claudogram illustrates the posterior distribution of species trees inferred with SNAPP based on the best species delimitation model PABSTE. High color density is indicative of areas in the species trees with high topology agreement. Different colors represent different topologies. The maximum clade credibility species tree is shown with thicker branches. Trees with the same topology as the maximum clade credibility species tree are colored in red. Trees with different topologies are colored green or blue. With the exception of the branch leading to the clade of P. johnsoni, P. maunga, and P. alisonae, which has a posterior probability of 0.87 , all interior branches have posterior probabilities of 1.0 .

All of our analyses based on RAD-seq matrices - varying in taxon coverage, degree of divergence among taxa, proportion of missing data, number of loci, and analysis type (concatenated or species tree) produced completely congruent trees, which together provide extremely high confidence on the phylogenetic hypothesis inferred for the octocoral AC clade (Figs. 1, 2 and 3). Consequently, we suggest that single marker gene trees in octocorals, particularly from the mitochondria, should not be considered as robust hypotheses of true species phylogenies on their own, without further validation by multiple 
informative and independent nuclear loci. We urge systematists to be conservative when making taxonomic rearrangements based on inferences from single-marker data alone.

\section{RAD-seq data is suitable for phylogenetic inference in divergent taxa}

Contrary to the currently accepted idea that RAD-seq data are only suitable for taxa with divergence times younger than 60 million years (MY) (Rubin et al. 2012), we demonstrate their suitability well beyond this age threshold. Remarkably, we were able to confidently resolve phylogenetic relationships among genera from different families diverging by at least $80 \mathrm{MY}$ in the $\mathrm{AC}$ clade. The split between the families Paragorgiidae and Coralliidae has been dated, using coralliid fossils, to be between 80-150 MY old (Ardila et al. 2012; Herrera et al. 2012). Park et al. (2012) estimated the age of the most recent common ancestor of the Coralliidae at approximately 50 MY (25-100 MY 95\% confidence region), using independent cnidarian fossils for molecular clock calibration. The split with the genera Anthomastus and Heteropolypus is likely older than $100 \mathrm{MY}$. It is without question that, due to evolution at restriction sites, the number of RAD loci among taxa for which orthology can be established decreases rapidly as divergence increases. However, we suggest that the suitability limits of RAD-seq for phylogenetics in divergent taxa cannot be assessed in terms absolute time, but depend on taxon-specific factors such as mutation rate, generation time and effective population size.

Bioinformatic studies addressing the issue of extent of the suitability of RAD-seq for phylogenetic inference have focused mainly on Drosophila as study model (Rubin et al. 2012; Cariou et al. 2013). Longer generation times and lower metabolic rates in taxa like deep-sea corals, relative to those in organisms like Drosophila, could cause a reduction in mutation rates (see review by Baer et al. (2007)), which may in turn decrease the evolutionary rates at restriction sites and allow for phylogenetic inferences using RAD-seq in situations of deeper divergence. Consistent with this hypothesis, we observe a nucleotide diversity $(\pi)$ calculated across all octocoral specimens from the PHYLO matrix of $0.012 \pm$ 0.002 (considered a minimum since RAD-seq can underestimate diversity (Arnold et al. 2013); see Table S5 and Table S6 for individual values), which is significantly lower than the nucleotide diversity in many of the Drosophila species included in the bioinformatic studies by Cariou et al. (2013) and Rubin et al. (2012). Nonetheless, there are other important factors known to influence genetic diversity across species - and likely the evolutionary rate as well. These factors include the effective population size, selection, habitat kind, geographic range, and mating system (Leffler et al. 2012). To sum up, we argue that RADseq can be successfully used to infer phylogenetic relationships in certain taxa with deeper divergences than previously suggested. This is particularly true when the number of RAD loci is maximized through 
the choice of restriction enzymes with higher cutting frequencies in the target taxon (Herrera et al. Chapter 2).

\section{RAD-seq allows the formulation of robust species delineations}

Our study, the first statistical rigorous test of species hypothesis in octocorals, provides conclusive evidence rejecting the current morphological species delimitation model for the genus Paragorgia. We find decisive support for a nested model that combines species boundaries from morphological taxonomy with cryptic diversity linked to environmental variables of geographic location and depth (Figs. 3 and 4). This nested model, proposes 9 species among the examined specimens. Five of these species correspond to the morphological species P. coralloides, P. kaupeka, P. alisonae, P. johnsoni, and P. maunga. Two splits, corresponding to sub-clades in the morphological species P. arborea and in P. stephencairnsi, indicate cases of cryptic species.

Herrera et al. (2012) found significant genetic differentiation of the north Pacific populations of $P$. arborea relative to the south Pacific, Atlantic and Indian ocean populations, and suggested that these populations may represent sub-species. The north Pacific populations of $P$. arborea were previously defined as a separate species, P. pacifica, by Verrill (1922) based on gross colony morphology, but later combined into a single species by Grasshoff (1979). Sánchez (2005) suggested potential small differences in medullar sclerite sizes and ornamentation between north Pacific specimens and specimens form elsewhere. However, we were unable to recognize these morphological differences in the few examined specimens in this study. Nonetheless, based on the decisive support for the split of $P$. arborea from analysis of genome-wide SNP makers indicates, we resurrect the species Paragorgia pacifica for the north Pacific populations of formerly P. arborea. We find no evidence of cryptic speciation between the north Atlantic and south Pacific P. arborea and therefore conclude it should be considered a single species as previously suggested by Herrera et al. (2012).

Depth is an important factor contributing to genetic differentiation and formation of species in the ocean, both shallow (Carlon \& Budd 2002; Prada \& Hellberg 2013) and deep (Miller et al. 2011; Jennings et al. 2013; Quattrini et al. 2013; Glazier \& Etter 2014). The observed cryptic differentiation between specimens of P. stephencairnsi collected shallower than $350 \mathrm{~m}$ and deeper than $1000 \mathrm{~m}$ indicates that depth is also a diversifying force in octocorals from the AC clade, which had gone undetected due to the low variability of traditional sequence data (Herrera et al. 2012). The holotype of $P$. stephencairnsi was collected from approximately $350 \mathrm{~m}$ in the Georgia Strait of British Columbia, overlapping in depth range and geographic region with that of most of the specimens from the shallow sub-clade examined in this 
study. Therefore, we propose to conserve that name P. stephencairnsi for that shallow sub-clade, and consider the deep sub-clade as a new species.

Other recent species delimitation studies in anthozoan corals have also revealed significant incongruences when comparing morphological and single-locus species delimitation hypotheses (particularly from mitochondrial data) with phylogenetic evidence from multi-locus datasets (Pante et al. 2014; Prada et al. 2014). In line with the findings of Pante et al. (2014), we find that specimens of Paragorgia sharing identical $m t M u t S$ haplotypes can belong to more than one species. Contrastingly, Herrera et al. (2012) present strong evidence showing significant mitochondrial haplotype diversity in the south Pacific and north Atlantic populations of Paragorgia arborea. Our observations, together with those from the aforementioned studies, constitute compelling evidence indicating that there is no solid basis for the widespread assumption that $m t M u t S$ haplotypes may be equivalent to individual octocoral species, as proposed by Thoma et al. (2009). The analysis with RAD-seq, or alternative genomic multi-locus methods, of a larger number of specimens from diverse geographic locations and depth horizons will likely reveal further cryptic diversity not characterized by mitochondrial haploytypes (see Fig. S2, Fig. S3, and Table S8), and thus further illuminates taxonomy and systematics in this an other groups.

\section{CONCLUSIONS}

In this case study we demonstrate the empirical utility of RAD-seq to resolve phylogenetic relationships among divergent and recalcitrant taxa and to objectively infer species boundaries by testing alternative delimitation hypotheses. We were able to make use of RAD-seq to overcome long-standing technical difficulties in octocoral genetics, and to resolve fundamental questions in species definitions and systematics. We show that classic morphological taxonomy can greatly benefit from integrative approaches that provide objective tests to species delimitation hypothesis. Our results pave the way for addressing further questions in biogeography, species ranges, community ecology, population dynamics and evolution of octocorals and other marine taxa. The results from this study also represent a valuable reference resource for the development of tools, such as SNP arrays, that can be used to perform accurate species identifications, and generate species inventories that will aid the design and implementation of conservation and management policies. 


\section{METHODS}

To perform identifications using current morphological species descriptions we performed colony observations and scanning electron microscopy of sclerites on 44 octocoral specimens from the AC clade (Table S1).

To obtain a genome-wide set of markers that could be useful for phylogenetic inferences of deepdivergent taxa and species delimitation in the AC clade (greater than 100 million years) we performed RAD sequencing with a the 6-cutter restriction enzyme PstI, which is predicted to cut between 32,000 and 110,000 times in the genome of an octocoral (Table S7). This predicted range was obtained using the observed frequency of the PstI recognition sequence, and its probability calculated using a trinucleotide composition model, in the genomes of the cnidarians Nematostella vectensis, Acropora digitifera, Hydra vulgaris, and Alatina moseri (Herrera et al. Chapter 2). Genome size range of 0.3-0.5 pg was used based on observations obtained through flow cytometry in gorgoniid octocorals by Luisa Dueñas at the Universidad de los Andes, Bogotá, Colombia (personal communication). Total genomic DNA was purified from specimens following protocols described in Herrera et al. (Chapter 3). Concentrationnormalized genomic DNA was submitted to Floragenex Inc (Eugene, OR). for library preparation and RAD sequencing. Libraries were sequenced by 48-multiplex, using 10-base pair barcodes, on a single lane of an Illumina Hi-Seq 2000 sequencer.

To compare the inferences obtained from RAD-seq data with the inferences drawn from traditional genetic barcoding data, we performed targeted sequencing of the mitochondrial mtMutS gene - a genetic marker widely used for phylogenetics and species delimitation studies in octocorals. Polymerase chain reactions were carried out following the protocols by Herrera et al. (Chapter 3). Primer pairs used for amplifications were AnthoCorMSH (Herrera et al. 2010) and Mut-3458R (Sánchez et al. 2003). Negative controls were included in every experiment to test for contamination. Purified PCR products were submitted to Eurofins Genomics (Eurofins MWG Operon, Inc.) for sequencing.

\section{RAD-seq data filtering}

Sequence reads were de-multiplexed and quality filtered with the process_radtags program from the package Stacks v1.20 (Catchen et al. 2013). Barcodes and Illumina adapters were excluded from each read and length was truncated to $91 \mathrm{bp}$ (-t 91) Reads with ambiguous bases were discarded (-c). Reads with an average quality score below 10 (-s 10) within a sliding window of $15 \%$ of the read length (-w $0.15)$ were discarded (-r). The rescue barcodes and RAD-tags algorithm was enabled (-r). Additional 
filtering, and the clustering within and between individuals to identify loci was performed using the program pyRAD v2.01 (Eaton 2014). Reads with more than 33 bases with a quality score below 20 were discarded.

\section{RAD-seq loci clustering and phylogenetic inference}

We investigated different combinations of clustering thresholds (c 0.80, 0.85 and 0.90) and minimum

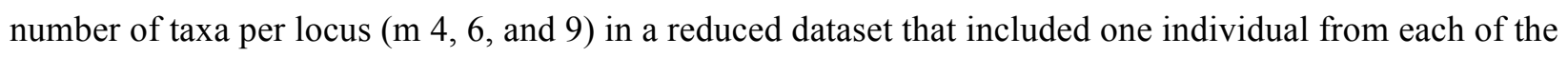
12 putative morphological species. The minimum depth of coverage required to build a cluster and the maximum number of shared polymorphic sites in a locus were kept constant at 4 (d) and 3 (p) respectively. Loci sequences were concatenated into combined matrices. We refer to these 9 resulting matrices as the 'backbone' matrices. Each of the resulting backbone matrices was analyzed in RAxMLHPC2 v8.0 (Stamatakis 2006) for maximum likelihood (ML) phylogenetic tree inference. For this, and all the other phylogenetic analyses in RAxML, we assumed a generalized time-reversible DNA substitution model with a gamma-distributed rate variation across sites (GTR GAMMA). Branch support was assessed by 500 bootstrap replicates.

We selected an optimal combination of loci clustering parameters as the set of parameters that minimized the number of missing data and maximized the number of phylogenetically informative sites while producing a highly supported phylogenetic tree. The optimal set of parameters chosen was a clustering threshold of $80 \%$ similarity among sequences (c 0.80 ) and a minimum coverage of taxa per locus of $75 \%$ (m 9). A concatenated matrix containing the sequence data of all the 44 octocoral specimens, denominated 'PHYLO', was built using this parameter combination (c 0.80, m 33) in pyRAD and subsequently analyzed in RAxML.

\section{Phylogenetic inference with traditional genetic barcoding data}

To compare the tree topology obtained from the phylogenetic inferences of the PHYLO RAD-seq dataset with traditional genetic barcoding data we analyzed the 'mitochondrial' dataset (containing the $m t M u t S$ sequences) using RAxML. These two datasets - PHYLO and mitochondrial - contain data from the same individuals. To place the specimens from this study in a broader phylogenetic context we also analyzed the mitochondrial dataset in RAxML with the addition of $m t M u t S$ data from 233 additional specimens belonging to the AC clade, as well as outgroups (see Table S8, Fig. S2, and Fig. S3).

\section{Testing species delimitation models for Paragorgia}


We constructed 5 taxonomy-guided species delimitation models for Paragorgia: i) 'morphid' model: 7 species based on current morphological species descriptions (Sánchez 2005); ii) 'PAB' model: 8 species based on the 7 morphological species plus a split of $P$. arborea based on previous evidence of genetic differentiation of north Pacific populations (Herrera et al. 2012); iii) 'STE' model: 8 species based on the 7 morphological species plus a split of $P$. stephencairnsi based on depth differences (specimens collected $<350 \mathrm{~m}$ vs. $>1000 \mathrm{~m}$ ), as depth is known to be an important structuring variable in marine taxa (Jennings et al. 2013; Prada \& Hellberg 2013; Quattrini et al. 2013); iv) 'PABSTE' model: 9 species based on the 7 morphological species plus the splits of the PAB and STE models; v) 'splitPAB' model: 10 species based on the 7 morphological species plus the split of the STE model and an additional split in the PAB model where $P$. arborea is split in 3 species corresponding to the ocean basin where the specimens were collected (north Pacific, south Pacific and north Atlantic).

We also generated taxonomy-unguided species delimitation models for Paragorgia through Bayesian and ML implementations of the Poisson tree processes model (PTP) (available at http://species.h-its.org/ptp/). PTP estimates the number of speciation events in a rooted phylogenetic tree in terms of nucleotide substitutions (Zhang et al. 2013). We used PTP to analyze the trees obtained from phylogenetic inferences in RAxML of reduced $m t M u t S$ and RAD-seq datasets that include only members of the family Paragorgiidae (genera Paragorgia and Sibogagorgia). The 'PARAGORGIIDAE' RAD-seq concatenated matrix was generated in pyRAD using a clustering threshold of $80 \%$ similarity among sequences (c 0.80 ) and a minimum coverage of taxa per locus of $100 \%(\mathrm{~m} \mathrm{33})$. The resulting phylogenetic trees of Paragorgia were rooted with the specimens of Sibogagorgia and analyzed by the PTP method using a Markov Chain Monte Carlo (MCMC) chain length of 500,000 generations (100 thinning, 25\% burnin). We assessed convergence by examining the likelihood trace. The combinations of the ML or Bayesian PTP implementations (mlPTP and bPTP, respectively) with the $m t M u t S$ or RAD-seq trees of Paragorgia resulted in four species delimitation models: i) 'mIPTPmt' model; ii) 'bPTPmt' model; iii) 'mIPTPrad' model; and iv) 'bPTPrad' model. Lastly, because deep-sea corals are known to show genetic differentiation at ocean basin/regional scales (Miller et al. 2011; Morrison et al. 2011; Herrera et al. 2012), we constructed an additional taxonomy-unguided species delimitation model - the 'geo' model - based on the geographic location where the specimens were collected (north Pacific, south Pacific or north Atlantic ocean basins).

To estimate the marginal likelihood of each species delimitation model we generated a matrix including only specimens of Paragorgia, denominated 'PARAGORGIA' using a clustering threshold of $80 \%$ similarity among sequences (c 0.80 ) and a minimum coverage of taxa per locus of $100 \%$ (m 31 ) in 
pyRAD. In contrast to the backbone, PHYLO, and PARAGORGIIDAE RAD-seq matrices, this matrix contained the data of one SNP per locus and not the entire locus sequence. We analyzed these data using the implementation of BFD* in the SNAPP (Bryant et al. 2012) plug-in for the program BEAST v2.1.3 (Bouckaert et al. 2014). We performed a path-sampling of 48 steps, with a MCMC chain length of 100,000 (10,000 pre-burnin), following the guidelines from Leache et al. (2014). Bayesian factors were calculated from the marginal likelihood estimates for each model and compared using the framework proposed by Kass and Raftery (1995)

\section{Species tree inference}

To test the tree topology in the genus Paragorgia obtained by the phylogenetic analysis of the PHYLO and PARAGORGIIDAE concatenated matrices we performed a species tree inference from the SNP data in the PARAGORGIA matrix using the program SNAPP. This program allows the inference of species trees from unlinked SNP data (only one SNP per locus retained) bypassing the inference of individual gene trees (Bryant et al. 2012). We performed 3 independent runs using a MCMC chain length of 10,000,000 (sampling every 1,000 generations; pre-burnin of 1,000) with default prior distributions for coalescence rate, mutation rate and ancestral population size parameters. We assessed convergence to stationary distributions and effective sample sizes $>200$ after $10 \%$ burnin in the program TRACER (Rambaut \& Drummond 2007). Species trees in the posterior distribution were summarized with the program DENSITREE v2.01 (Bouckaert 2010).

\section{ACKNOWLEDGEMENTS}

This research was supported by the National Geographic Society/Waitt Foundation (W285-13 to SH); the National Oceanic and Atmospheric Administration (NA09OAR4320129 to TS); the National Science Foundation (OCE-1131620 to TS); the National Aeronautics and Space Administration (NNX09AB76G to TS); and the Academic Programs Office (Ocean Ventures Fund to SH), the Ocean Exploration Institute (Fellowship support to TMS) and the Ocean Life Institute of the Woods Hole Oceanographic Institution (WHOI).

Specimens provided by the National Institute of Water and Atmospheric Research (NIWA) were collected under research programs: Kermadec Arc Minerals, funded by the New Zealand Ministry of Business, Innovation \& Employment (MBIE), Auckland University, Institute of Geological and Nuclear Science (GNS), and WHOI; Ocean Survey 20/20 funded by Land Information New Zealand; Impact of resource use on vulnerable deep-sea communities (CO1X0906), funded by MBIE; Nascent Inter-Ridge 
Volcanic And Neotectonic Activity, funded by the Ministry for Primary Industries (MPI), GNS, MBIE, and the U. of New Hampshire; Scientific Observer Program funded by MPI; and the Joint New ZealandUSA 2005 NOAA Ring of Fire Expedition, part of NIWA's Seamount Program (FRST CO1X0508). For enabling access to key specimens we thank K. Schnabel (NIWA), S. Mills (NIWA), D. Tracey (NIWA), M. Clark (NIWA), A. Rowden (NIWA), S. Cairns (Smithsonian), E. Cordes (Temple U.), A. Quattrini (Temple U.), G. Workman (Department of Fisheries and Oceans Canada - DFO), M. Wyeth (DFO), K. Anderson (DFO), M. Frey (Royal British Columbia Museum - RBCM), H. Gartner (RBCM), L. Watling (U. Hawaii), J. Adkins (CalTech). We thank P. Aldersdale (CSIRO), N. Ardila (ECOMAR) and J. Sanchez (U. Andes) for assistance with morphological identifications. We also thank E. O'Brien (WHOI), D. Forsman (WHOI), J. Fellows (WHOI), J. \& S. Schooner, K. Heylar, and N. McDaniel for invaluable assistance during scuba diving fieldwork in British Columbia (DFO scientific license FIN130270). We thank the chief scientists, masters, crew, scientific personnel, and funding agencies of expeditions AT0735, KOK0506, Lophelia II 2009, RB-0503, TAN1007, TAN1104, TAN1206, and TAN1213. We also thank A. Tarrant and A. Reitzel for providing helpful comments that improved this manuscript.

\section{REFERENCES}

Aguilar C, Sánchez JA (2007) Phylogenetic hypotheses of gorgoniid octocorals according to ITS2 and their predicted RNA secondary structures. Molecular Phylogenetics and Evolution 43, 774-786.

Ardila NE, Giribet G, Sanchez JA (2012) A time-calibrated molecular phylogeny of the precious corals: reconciling discrepancies in the taxonomic classification and insights into their evolutionary history. BMC Evolutionary Biology 12.

Arnold B, Corbett-Detig RB, Hartl D, Bomblies K (2013) RADseq underestimates diversity and introduces genealogical biases due to nonrandom haplotype sampling. Molecular Ecology 22, 3179-3190.

Baer CF, Miyamoto MM, Denver DR (2007) Mutation rate variation in multicellular eukaryotes: causes and consequences. Nature Reviews Genetics 8, 619-631.

Baird NA, Etter PD, Atwood TS, et al. (2008) Rapid SNP discovery and genetic mapping using sequenced RAD markers. PLoS One 3, 3376.

Bayer FM (1956) Octocorallia. In: Treatise on Invertebrate Paleontology Part F. Coelenterata (ed. Moore RC), pp. 163-231. Geological Society of America and University of Kansas Press, Lawrence, Kansas.

Berntson EA, Bayer FM, McArthur AG, France SC (2001) Phylogenetic relationships within the Octocorallia (Cnidaria: Anthozoa) based on nuclear 18S rRNA sequences. Marine Biology 138, 235-246.

Bilewitch JP, Degnan SM (2011) A unique horizontal gene transfer event has provided the octocoral mitochondrial genome with an active mismatch repair gene that has potential for an unusual selfcontained function. BMC Evolutionary Biology 11, 228.

Bouckaert R, Heled J, Kuhnert D, et al. (2014) BEAST 2: a software platform for Bayesian evolutionary analysis. PLoS Comput Biol 10, e1003537.

Bouckaert RR (2010) DensiTree: making sense of sets of phylogenetic trees. Bioinformatics 26, 13721373. 
Brockman SA, McFadden CS (2012) The mitochondrial genome of Paraminabea aldersladei (Cnidaria: Anthozoa: Octocorallia) supports intramolecular recombination as the primary mechanism of gene rearrangement in octocoral mitochondrial genomes. Genome Biology and Evolution 4, 9941006.

Bryant D, Bouckaert R, Felsenstein J, Rosenberg NA, RoyChoudhury A (2012) Inferring species trees directly from biallelic genetic markers: Bypassing gene trees in a full coalescent analysis. Molecular Biology and Evolution 29, 1917-1932.

Cairns S (2007) Deep-water corals: An overview with special reference to diversity and distribution of deep-water scleractinian corals. Bulletin of Marine Science 81, 311-322.

Cariou M, Duret L, Charlat S (2013) Is RAD-seq suitable for phylogenetic inference? An in silico assessment and optimization. Ecology and Evolution 3, 846-852.

Carlon DB, Budd AF (2002) Incipient speciation across a depth gradient in a scleractinian coral? Evolution 56, 2227-2242.

Catchen J, Hohenlohe PA, Bassham S, Amores A, Cresko WA (2013) Stacks: an analysis tool set for population genomics. Molecular Ecology 22, 3124-3140.

CITES (2014) Appendices I, II and III. Convention on International Trade in Endangered Species of wild fauna and flora, , http://www.cites.org/sites/default/files/eng/app/2014/E-Appendices-2014-0914.pdf.

Clark AG, Eisen MB, Smith DR, et al. (2007) Evolution of genes and genomes on the Drosophila phylogeny. Nature 450, 203-218.

Concepcion GT, Crepeau MW, Wagner D, Kahng SE, Toonen RJ (2007) An alternative to ITS, a hypervariable, single-copy nuclear intron in corals, and its use in detecting cryptic species within the octocoral genus Carijoa. Coral reefs 27, 323-336.

Cruaud A, Gautier M, Galan M, et al. (2014) Empirical assessment of RAD sequencing for interspecific phylogeny. Molecular Biology and Evolution 31, 1272-1274.

Daly M, Brugler MR, Cartwright P, et al. (2007) The phylum Cnidaria: A review of phylogenetic patterns and diversity 300 years after Linnaeus. Zootaxa 1668, 127-182.

De Queiroz K (2007) Species concepts and species delimitation. Systematic Biology 56, 879-886.

Dobzhansky T (1970) Genetics of the Evolutionary Process Columbia University Press, New York,.

Dueñas LF, Alderslade P, Sánchez JA (2014) Molecular systematics of the deep-sea bamboo corals (Octocorallia: Isididae: Keratoisidinae) from New Zealand with descriptions of two new species of Keratoisis. Molecular Phylogenetics and Evolution 74, 15-28.

Dueñas LF, Sánchez JA (2009) Character lability in deep-sea bamboo corals (Octocorallia, Isididae, Keratoisidinae). Marine Ecology Progress Series 397, 11-23.

Eaton DA (2014) PyRAD: Assembly of de novo RADseq loci for phylogenetic analyses. Bioinformatics 30, 1844-1849.

Eaton DAR, Ree RH (2013) Inferring phylogeny and introgression using RADseq data: An example from flowering plants (Pedicularis: Orobanchaceae). Systematic Biology 62, 689-706.

Edwards SV (2009) Is a new and general theory of molecular systematics emerging? Evolution 63, 1-19.

Emerson KJ, Merz CR, Catchen JM, et al. (2010) Resolving postglacial phylogeography using highthroughput sequencing. Proceedings of the National Academy of Sciences of the United States of America 107, 16196-16200.

Escudero M, Eaton DAR, Hahn M, Hipp AL (2014) Genotyping-by-sequencing as a tool to infer phylogeny and ancestral hybridization: A case study in Carex (Cyperaceae). Molecular Phylogenetics and Evolution 79, 359-367.

Figueroa DF, Baco AR (2014) Complete mitochondrial genomes elucidate phylogenetic relationships of the deep-sea octocoral families Coralliidae and Paragorgiidae. Deep-Sea Research Part Ii-Topical Studies in Oceanography 99, 83-91.

France SC (2007) Genetic analysis of bamboo corals (Cnidaria : Octocorallia : Isididae): Does lack of colony branching distinguish Lepidisis from Keratoisis? In: Bulletin of Marine Science, pp. 323333. 
France SC, Hoover LL, Hoover LL (2002) DNA sequences of the mitochondrial COI gene have low levels of divergence among deep-sea octocorals (Cnidaria: Anthozoa). Hydrobiologia 471, 149155.

Fujisawa T, Barraclough TG (2013) Delimiting species using single-locus data and the generalized mixed Yule coalescent approach: A revised method and evaluation on simulated data sets. Systematic Biology 62, 707-724.

Glazier AE, Etter RJ (2014) Cryptic speciation along a bathymetric gradient. Biological Journal of the Linnean Society 113, 897-913.

Grajales A, Aguilar C, Sánchez JA (2007) Phylogenetic reconstruction using secondary structures of Internal Transcribed Spacer 2 (ITS2, rDNA): finding the molecular and morphological gap in Caribbean gorgonian corals. BMC Evolutionary Biology 7, 90.

Grasshoff M (1979) Zur bipolaren verbreitung der oktokoralle Paragorgia arborea (Cnidaria: Anthozoa: Scleraxonia). Senckenbergiana Maritima 11, 115-137.

Grummer JA, Bryson RW, Reeder TW (2014) Species delimitation using Bayes factors: Simulations and application to the Sceloporus scalaris species group (Squamata: Phrynosomatidae). Systematic Biology 63, 119-133.

Hebert PDN, Cywinska A, Ball SL, DeWaard JR (2003) Biological identifications through DNA barcodes. Proceedings of the Royal Society B-Biological Sciences 270, 313-321.

Hellberg ME (2006) No variation and low synonymous substitution rates in coral mtDNA despite high nuclear variation. BMC Evolutionary Biology 6.

Herrera S, Baco A, Sánchez JA (2010) Molecular systematics of the bubblegum coral genera (Paragorgiidae, Octocorallia) and description of a new deep-sea species. Molecular Phylogenetics and Evolution 55, 123-135.

Herrera S, Reyes-Herrera PH, Shank TM (Chapter 2) Genome-wide predictability of restriction sites across the eukaryotic tree of life.

Herrera S, Shank TM, Sánchez JA (2012) Spatial and temporal patterns of genetic variation in the widespread antitropical deep-sea coral Paragorgia arborea. Molecular Ecology 21, 6053-6067.

Herrera S, Watanabe H, Shank T (Chapter 3) Evolutionary and biogeographical patterns of barnacles from deep-sea hydrothermal vents.

Hipp AL, Eaton DAR, Cavender-Bares J, et al. (2014) A framework phylogeny of the american oak clade based on sequenced RAD data. PLoS One 9, e93975.

ICES (2013) Assessment of the list of VME indicator species and elements. International Council for the Exploration of the Sea, http://www.ices.dk/sites/pub/Publication Reports/Advice/2013/Special requests/NEAFC_VME indicator_species and elements.pdf.

Jennings RM, Etter RJ, Ficarra L (2013) Population differentiation and species formation in the deep sea: the potential role of environmental gradients and depth. PLoS One 8, e77594.

Jones JC, Fan S, Franchini P, Schartl M, Meyer A (2013) The evolutionary history of Xiphophorus fish and their sexually selected sword: a genome-wide approach using restriction site-associated DNA sequencing. Molecular Ecology 22, 2986-3001.

Kass RE, Raftery AE (1995) Bayes Factors. Journal of the American Statistical Association 90, 773-795.

Leache AD, Fujita MK, Minin VN, Bouckaert RR (2014) Species Delimitation using Genome-Wide SNP Data. Systematic Biology 63, 534-542.

Leffler EM, Bullaughey K, Matute DR, et al. (2012) Revisiting an old riddle: What determines genetic diversity levels within species? PLoS Biology 10, e1001388.

Maddison WP (1997) Gene trees in species trees. Systematic Biology 46, 523-536.

Mayr E (1942) Systematics and the Origin of Species from the Viewpoint of a Zoologist Columbia University Press, New York,

Mcfadden CS, Benayahu Y, Pante E, et al. (2010a) Limitations of mitochondrial gene barcoding in Octocorallia. Molecular Ecology Resources 11, 19-31. 
McFadden CS, France SC, Sánchez JA, Alderslade P (2006) A molecular phylogenetic analysis of the Octocorallia (Cnidaria: Anthozoa) based on mitochondrial protein-coding sequences. Molecular Phylogenetics and Evolution 41, 513-527.

McFadden CS, Sanchez JA, France SC (2010b) Molecular Phylogenetic Insights into the Evolution of Octocorallia: A Review. Integrative and Comparative Biology 50, 389-410.

Mcfadden CS, Tullis ID, Breton Hutchinson M, Winner K, Sohm JA (2004) Variation in Coding (NADH Dehydrogenase Subunits 2, 3, and 6) and Noncoding Intergenic Spacer Regions of the Mitochondrial Genome in Octocorallia (Cnidaria: Anthozoa). Marine Biotechnology 6, 516-526.

McFadden CS, van Ofwegen LP (2013) Molecular phylogenetic evidence supports a new family of octocorals and a new genus of Alcyoniidae (Octocorallia, Alcyonacea). Zookeys 346, 59-83.

Miller KJ, Rowden AA, Williams A, Haussermann V (2011) Out of their depth? Isolated deep populations of the cosmopolitan coral Desmophyllum dianthus may be highly vulnerable to environmental change. PLoS One 6, e19004.

Morrison CL, Ross SW, Nizinski MS, et al. (2011) Genetic discontinuity among regional populations of Lophelia pertusa in the North Atlantic Ocean. Conservation Genetics 12, 713-729.

Nadeau NJ, Martin SH, Kozak KM, et al. (2012) Genome-wide patterns of divergence and gene flow across a butterfly radiation. Molecular Ecology 22, 814-826.

Pante E, Abdelkrim J, Viricel A, Gey D, France S (2014) Use of RAD sequencing for delimiting species. Heredity.

Park E, Hwang DS, Lee JS, et al. (2012) Estimation of divergence times in cnidarian evolution based on mitochondrial protein-coding genes and the fossil record. Molecular Phylogenetics and Evolution 62, 329-345.

Pons J, Barraclough TG, Gomez-Zurita J, et al. (2006) Sequence-based species delimitation for the DNA taxonomy of undescribed insects. Systematic Biology 55, 595-609.

Prada C, DeBiasse MB, Neigel JE, et al. (2014) Genetic species delineation among branching Caribbean Porites corals. Coral Reefs 33, 1019-1030.

Prada C, Hellberg ME (2013) Long prereproductive selection and divergence by depth in a Caribbean candelabrum coral. Proceedings of the National Academy of Sciences of the United States of America 110, 3961-3966.

Quattrini AM, Georgian SE, Byrnes L, et al. (2013) Niche divergence by deep-sea octocorals in the genus Callogorgia across the continental slope of the Gulf of Mexico. Molecular Ecology 22, 41234140.

Rambaut A, Drummond AJ (2007) Tracer v1.4, Available from http://beast.bio.ed.ac.uk/Tracer.

Reitzel AM, Herrera S, Layden MJ, Martindale MQ, Shank TM (2013) Going where traditional markers have not gone before: utility of and promise for RAD sequencing in marine invertebrate phylogeography and population genomics. Molecular Ecology 22, 2953-2970.

Roberts JM, Cairns SD (2014) Cold-water corals in a changing ocean. Current Opinion in Environmental Sustainability 7, 118-126.

Roberts JM, Wheeler A, Freiwald AR, Cairns SD (2009) Cold-Water Corals : The Biology and Geology of Deep-Sea Coral Habitats Cambridge University Press, Cambridge, UK ; New York.

Rubin BE, Ree RH, Moreau CS (2012) Inferring phylogenies from RAD sequence data. PLoS One 7, e33394.

Sánchez JA (2005) Systematics of the bubblegum corals (Cnidaria: Octocorallia: Paragorgiidae) with description of new species from New Zealand and the Eastern Pacific. Zootaxa 1014, 1-72.

Sanchez JA, Dorado D (2008) Intragenomic ITS2 variation in Caribbean seafans. In: Proceedings of the 11th International Coral Reef Symposium, pp. 1383-1387, Ft. Lauderdale.

Sánchez JA, Lasker HR, Taylor DJ (2003) Phylogenetic analyses among octocorals (Cnidaria): mitochondrial and nuclear DNA sequences (lsu-rRNA, 16S and ssu-rRNA, 18S) support two convergent clades of branching gorgonians. Molecular Phylogenetics and Evolution 29, 31-42.

Seetharam AS, Stuart GW (2013) Whole genome phylogeny for 21 Drosophila species using predicted 2b-RAD fragments. PeerJ 1, e226. 
Shearer TL, Van Oppen MJH, Romano SL, Worheide G (2002) Slow mitochondrial DNA sequence evolution in the Anthozoa (Cnidaria). Molecular Ecology 11, 2475-2487.

Smith P, McVeagh S, Mingoia J, France S (2004) Mitochondrial DNA sequence variation in deep-sea bamboo coral (Keratoisidinae) species in the southwest and northwest Pacific Ocean. Marine Biology 144, 253-261.

Stamatakis A (2006) RAxML-VI-HPC: Maximum likelihood-based phylogenetic analyses with thousands of taxa and mixed models. Bioinformatics 22, 2688-2690.

Thoma J, Pante E, Brugler M, France S (2009) Deep-sea octocorals and antipatharians show no evidence of seamount-scale endemism in the NW Atlantic. Marine Ecology Progress Series 397, 25-35.

Uda K, Komeda Y, Fujita T, et al. (2013) Complete mitochondrial genomes of the Japanese pink coral (Corallium elatius) and the Mediterranean red coral (Corallium rubrum): a reevaluation of the phylogeny of the family Coralliidae based on molecular data. Comparative Biochemistry and Physiology D-Genomics \& Proteomics 8, 209-219.

Verrill AE (1922) Part G: Alcyonaria and Actiniaria. Report of the Canadian Arctic Expedition 1913-18 8, 1-164.

Wagner CE, Keller I, Wittwer S, et al. (2012) Genome-wide RAD sequence data provide unprecedented resolution of species boundaries and relationships in the Lake Victoria cichlid adaptive radiation. Molecular Ecology 22, 787-798.

Wating L, France SC, Pante E, Simpson A (2011) Biology of deep-water octocorals. Advances in Marine Biology, Vol 60 60, 41-122.

Yang ZH, Rannala B (2010) Bayesian species delimitation using multilocus sequence data. Proceedings of the National Academy of Sciences of the United States of America 107, 9264-9269.

Zapata F, Jimenez I (2012) Species delimitation: Inferring gaps in morphology across geography. Systematic Biology 61, 179-194.

Zhang J, Kapli P, Pavlidis P, Stamatakis A (2013) A general species delimitation method with applications to phylogenetic placements. Bioinformatics 29, 2869-2876.

\section{SUPPLEMENTARY MATERIALS}




\section{Minimum taxa coverage per locus}

4

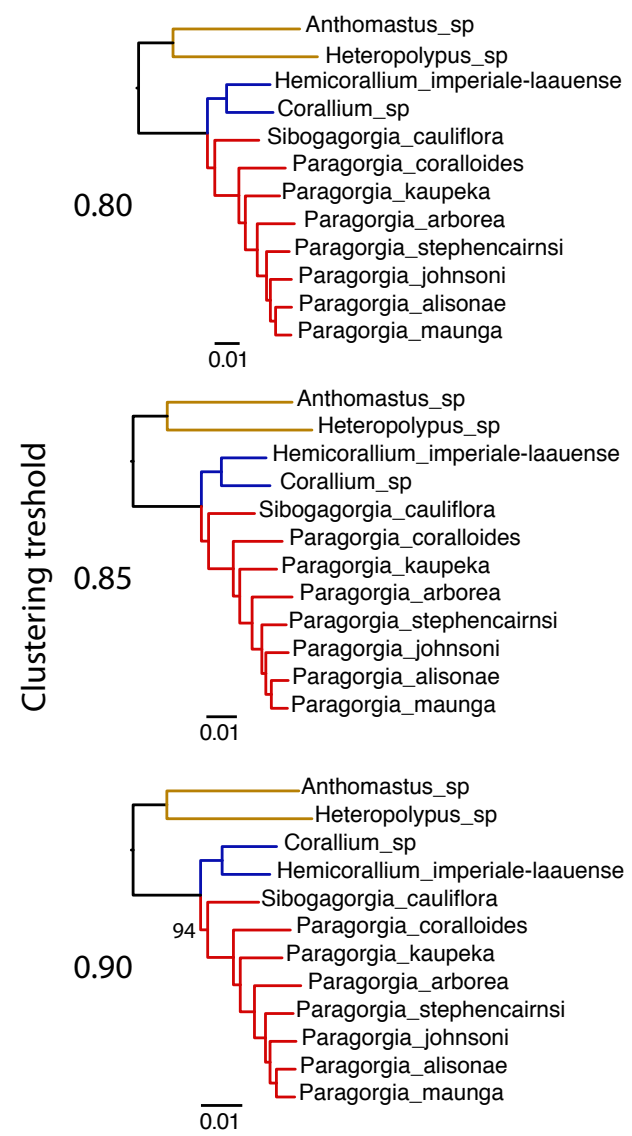

6

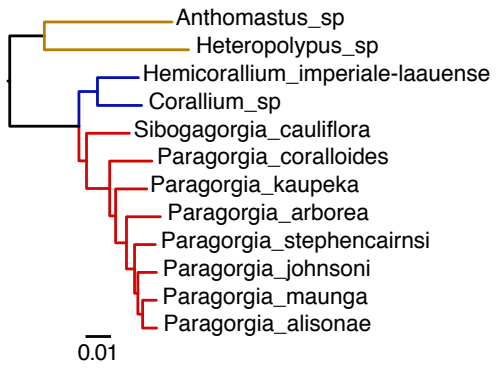
Anthomastus_sp
Corallium_sp
Coropolypus_sp
Semicorallium_imperiale-laauense
Sibogagorgia_cauliflora
Paragorgia_coralloides

_Paragorgia_kaupeka

_ Paragorgia_arborea _Paragorgia_stephencairnsi _-Paragorgia_johnsoni - Paragorgia_alisonae $\overline{0.01}$ LParagorgia_maunga

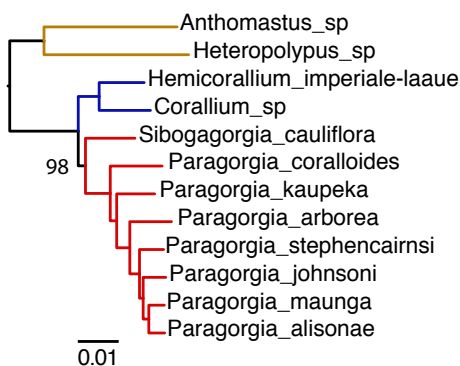

9

Anthomastus_sp _Heteropolypus_sp

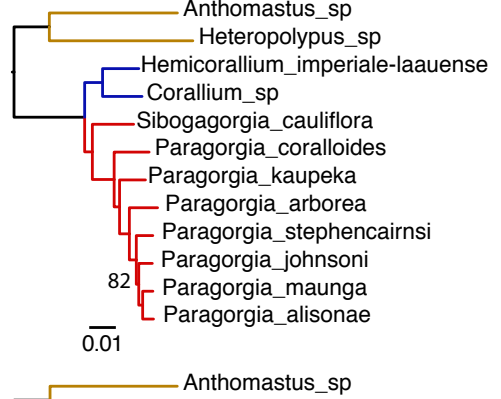
A Heteropolypus_sp

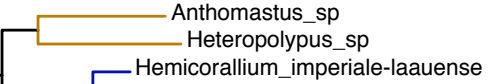

Corallium_sp

__Sibogagorgia_cauliflora _Paragorgia_coralloides _Paragorgia_kaupeka _ Paragorgia_arborea 4 -Paragorgia_stephencairnsi -Paragorgia_johnsoni 85 _Paragorgia_maunga $\overline{0.01}$ [Paragorgia_alisonae

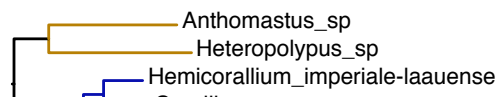
Anthomastus_sp Hemicorallium imperiale-laauense Corallium_sp 98 Sibogagorgia_cauliflora 98 - Paragorgia_coralloide _Paragorgia_kaupeka _ Paragorgia_arborea 4 Paragorgia_stephencairnsi -Paragorgia_johnsoni 82 Paragorgia_maunga $\overline{0.01}$

Figure S1. Phylogenetic trees based on backbone matrices. Trees inferred from the 9 backbone RAD-seq matrices built with different parameters of clustering threshold (c $0.80,0.85$ and 0.90 ; indicated by vertical labels) and

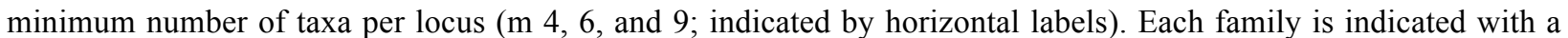
different branch color: red for Paragorgiidae; blue for Coralliidae; and yellow for Alcyoniidae. Trees were inferred with RAxML. All interior branches have bootstrap support values of 100, except for those shown. Scale bars indicate substitutions per site. 


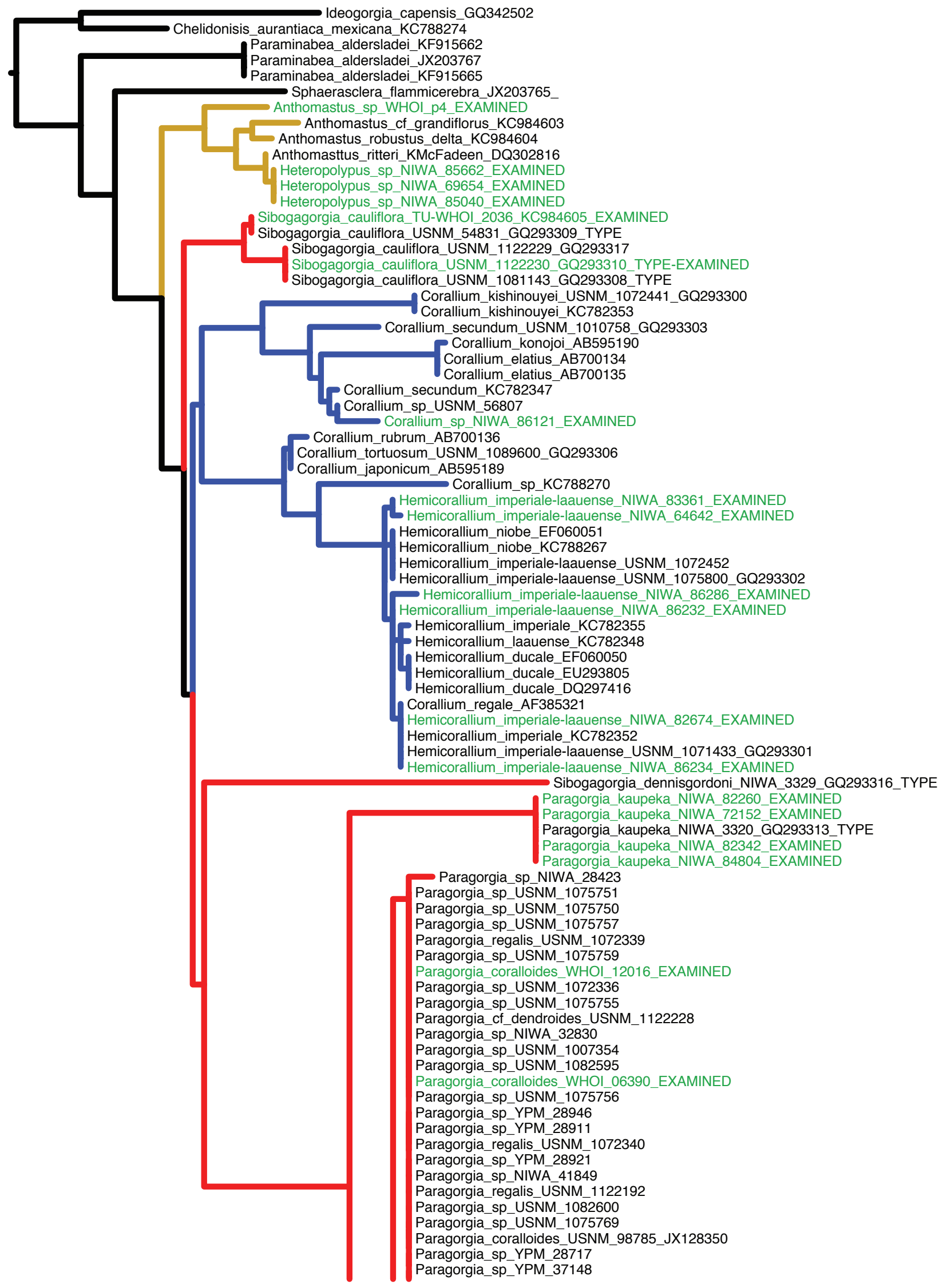




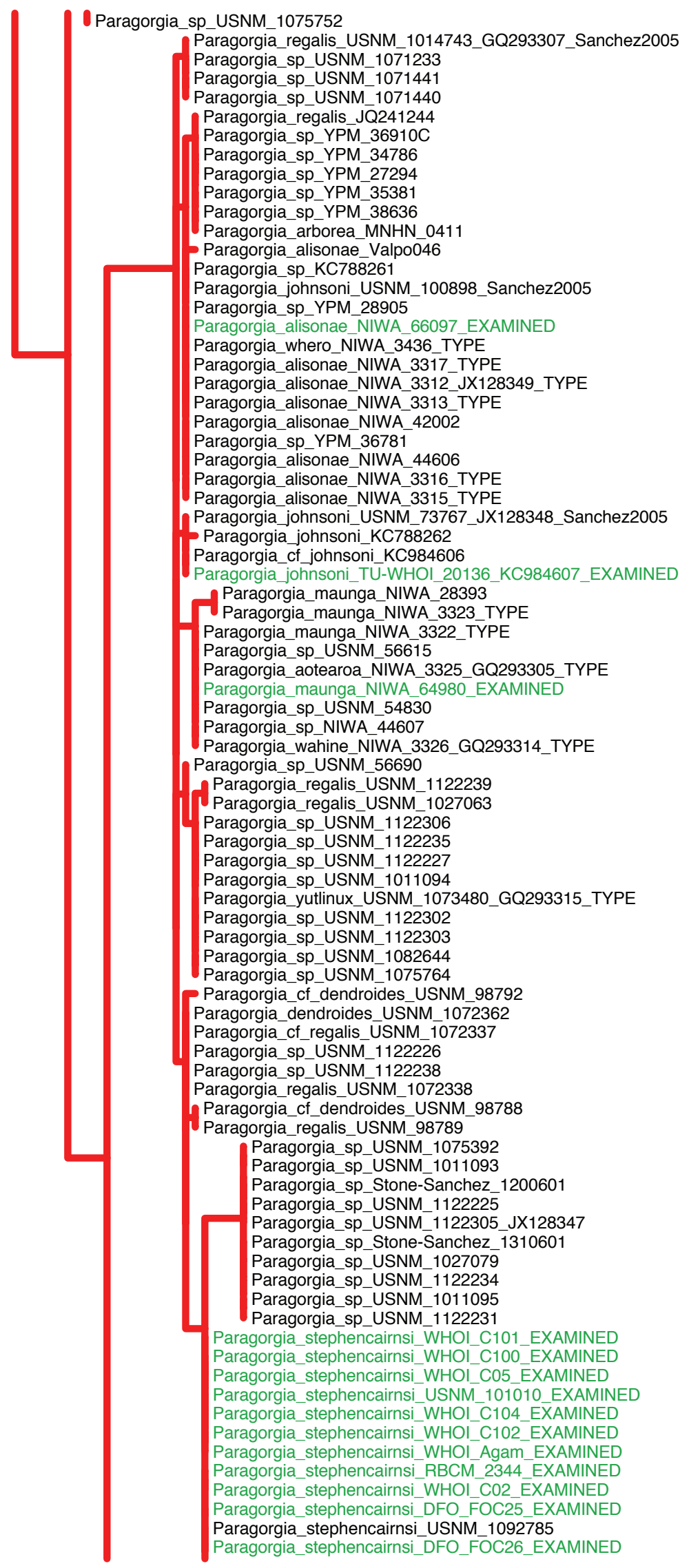




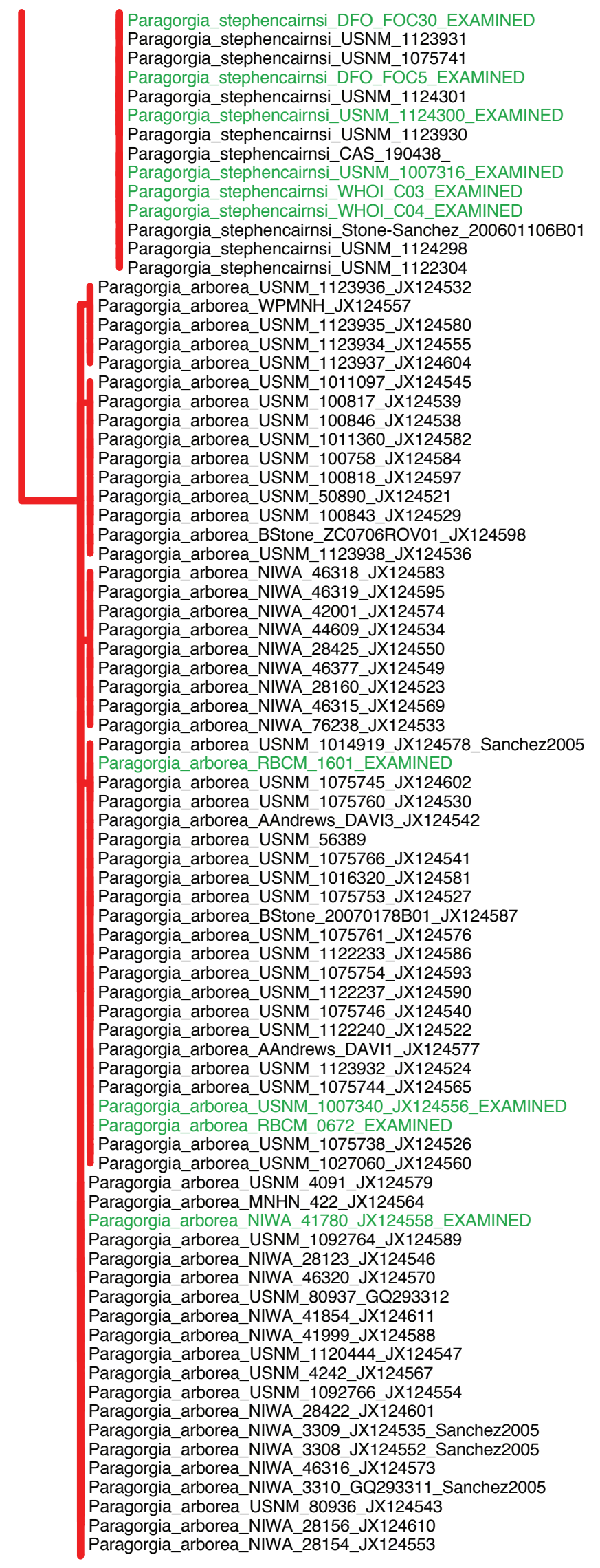




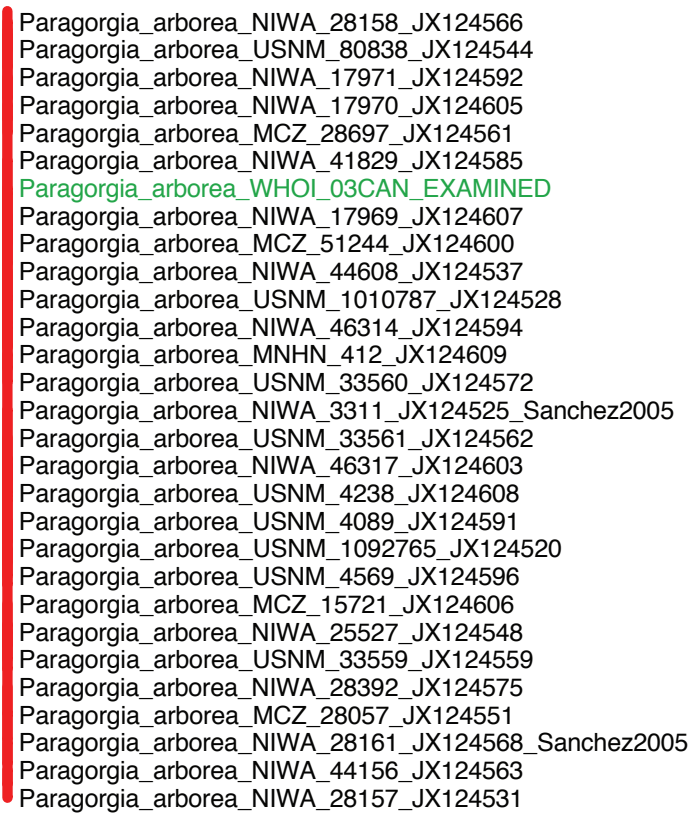

0.02

Figure S2. Mitochondrial $m t M u t S$ gene tree of all available sequences for the clade AC. Tree inferred from $m t M u t S$ sequence data from specimens examined in this study, GenBank, and additional specimens. Each family is indicated with a different branch color: red for Paragorgiidae; blue for Coralliidae; and yellow for Alcyoniidae. Outgroups are indicated with black branches. Specimens examined in detail in this study are indicated with green labels. Type specimens are labeled TYPE. Specimens examined in Sanchez (2005) are labeled "Sanchez2005". Tree was inferred with RAxML. Scale bars indicate substitutions per site. 


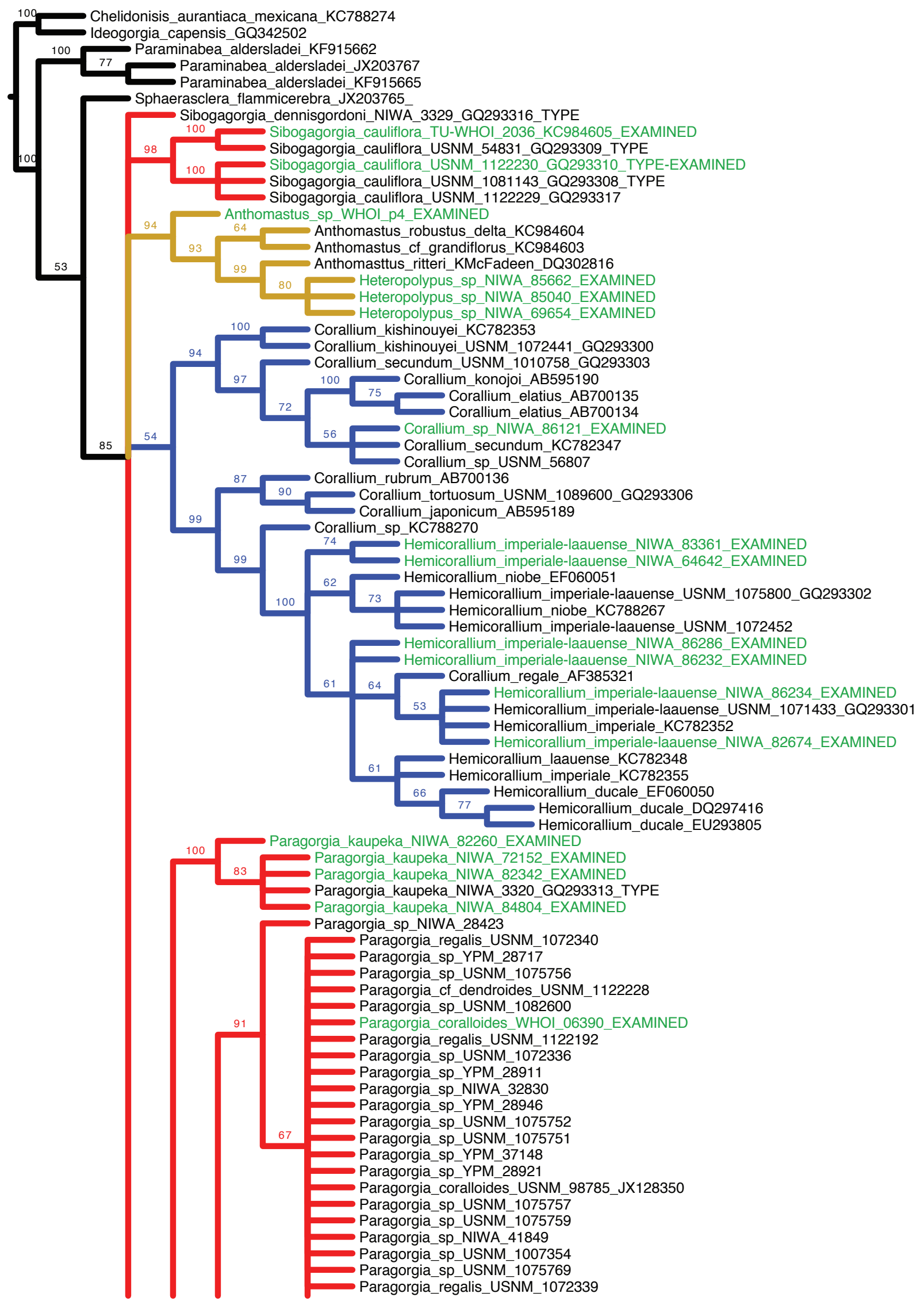




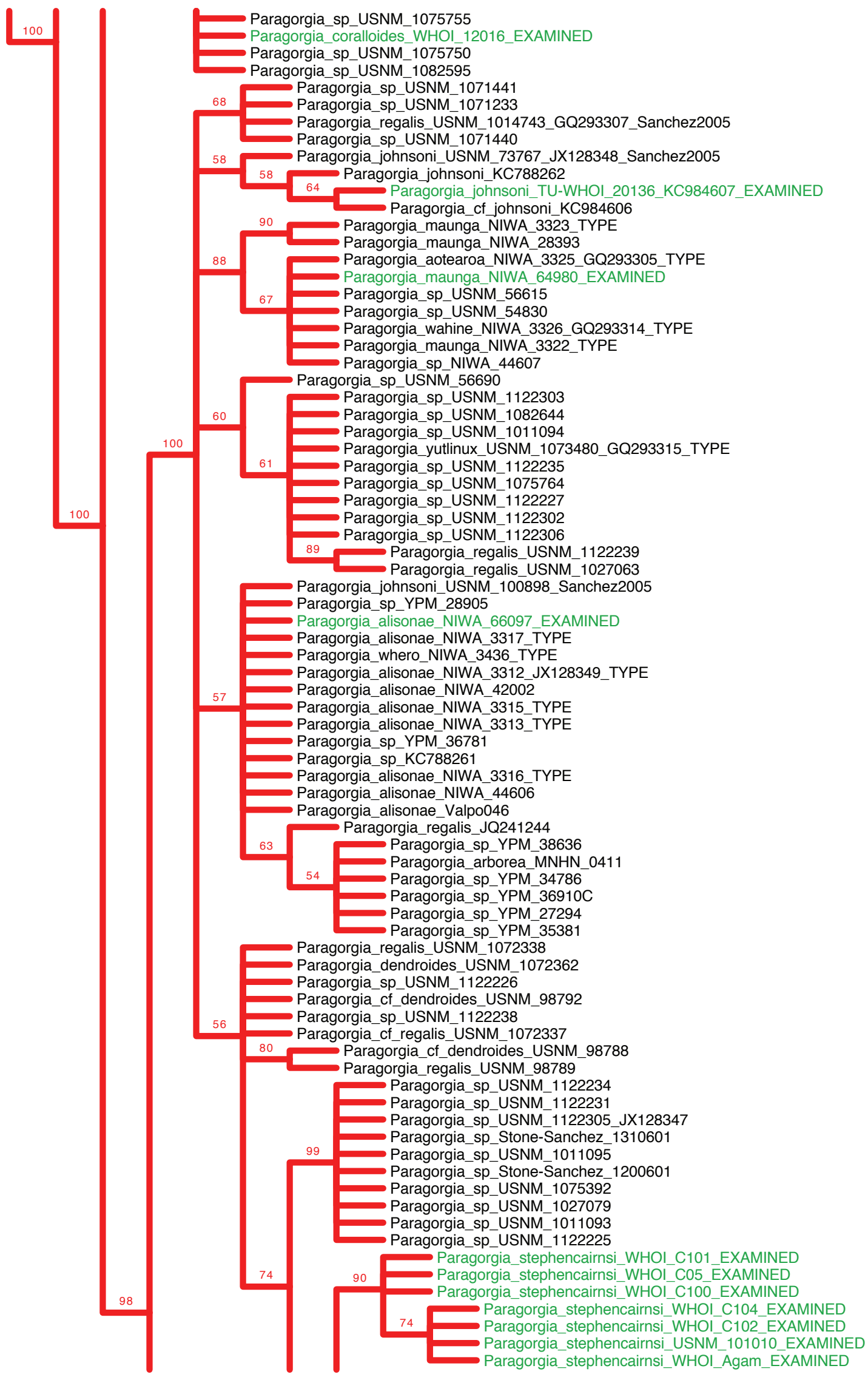




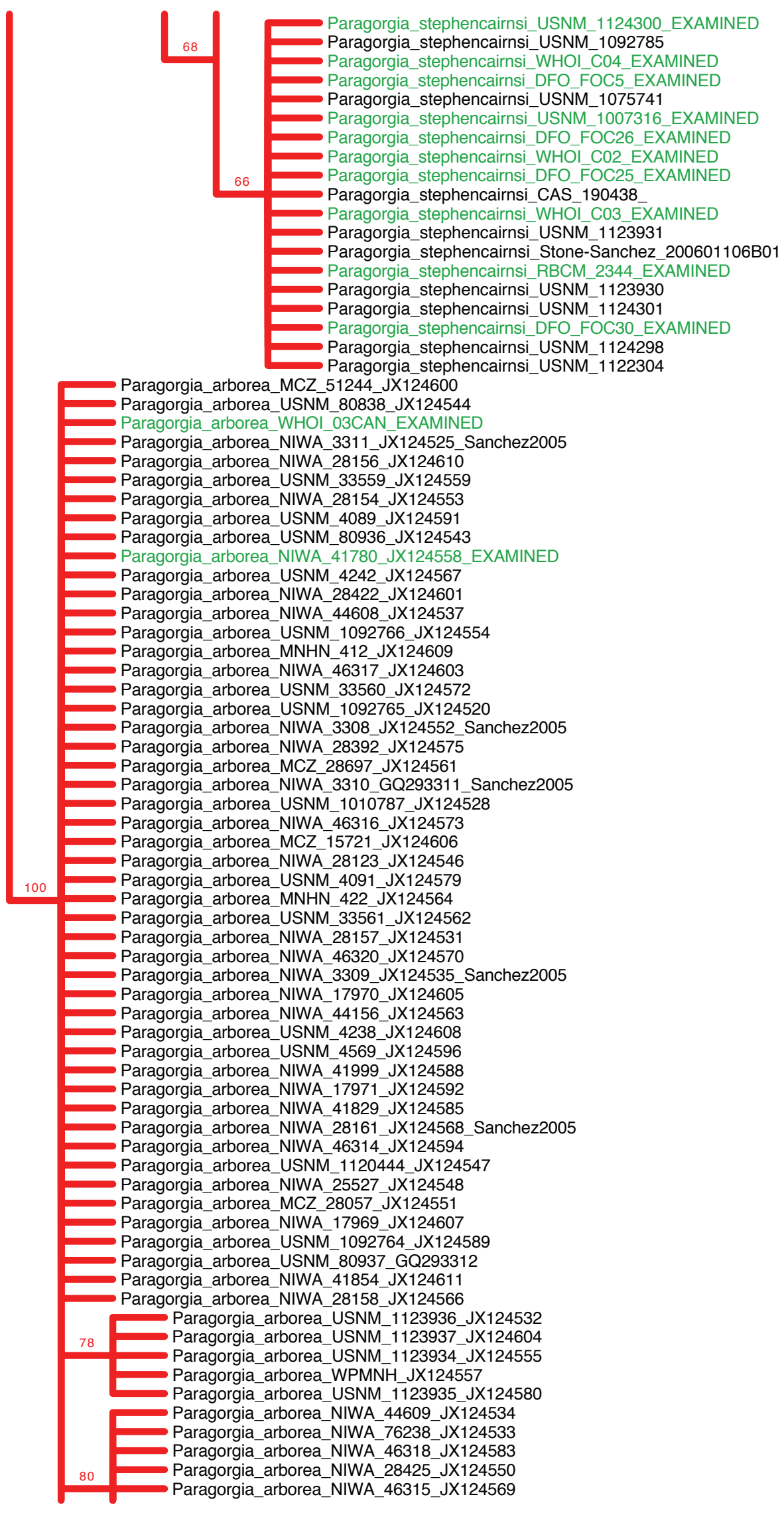




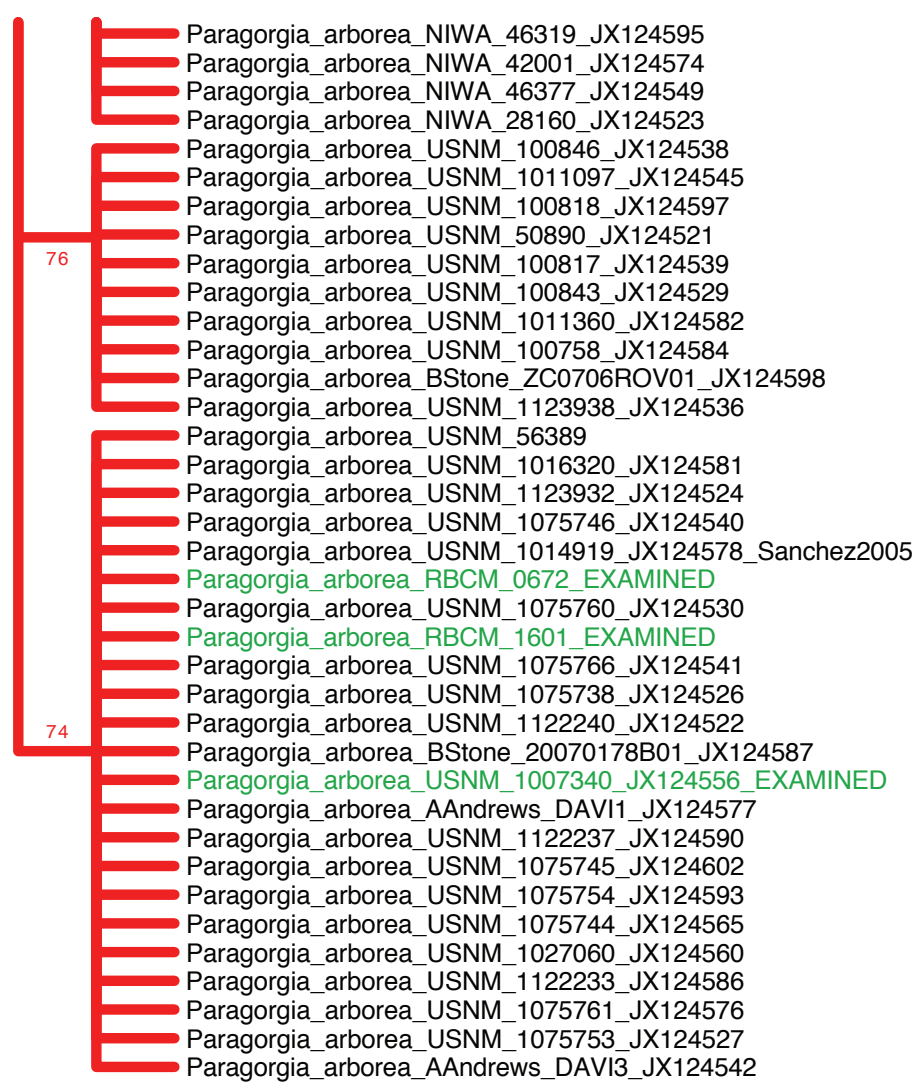

Figure S3. Mitochondrial $m t M u t S$ bootstrap support consensus tree of all available sequences for the clade AC. Tree inferred from mtMutS sequence data from specimens examined in this study, GenBank, and additional specimens. Each family is indicated with a different branch color: red for Paragorgiidae; blue for Coralliidae; and yellow for Alcyoniidae. Outgroups are indicated with black branches. Specimens examined in detail in this study are indicated with green labels. Type specimens are labeled "TYPE". Specimens examined in Sanchez (2005) are labeled "Sanchez2005". Tree was created with RAxML using a 50\% majority consensus from 500 bootstrap replicates. 


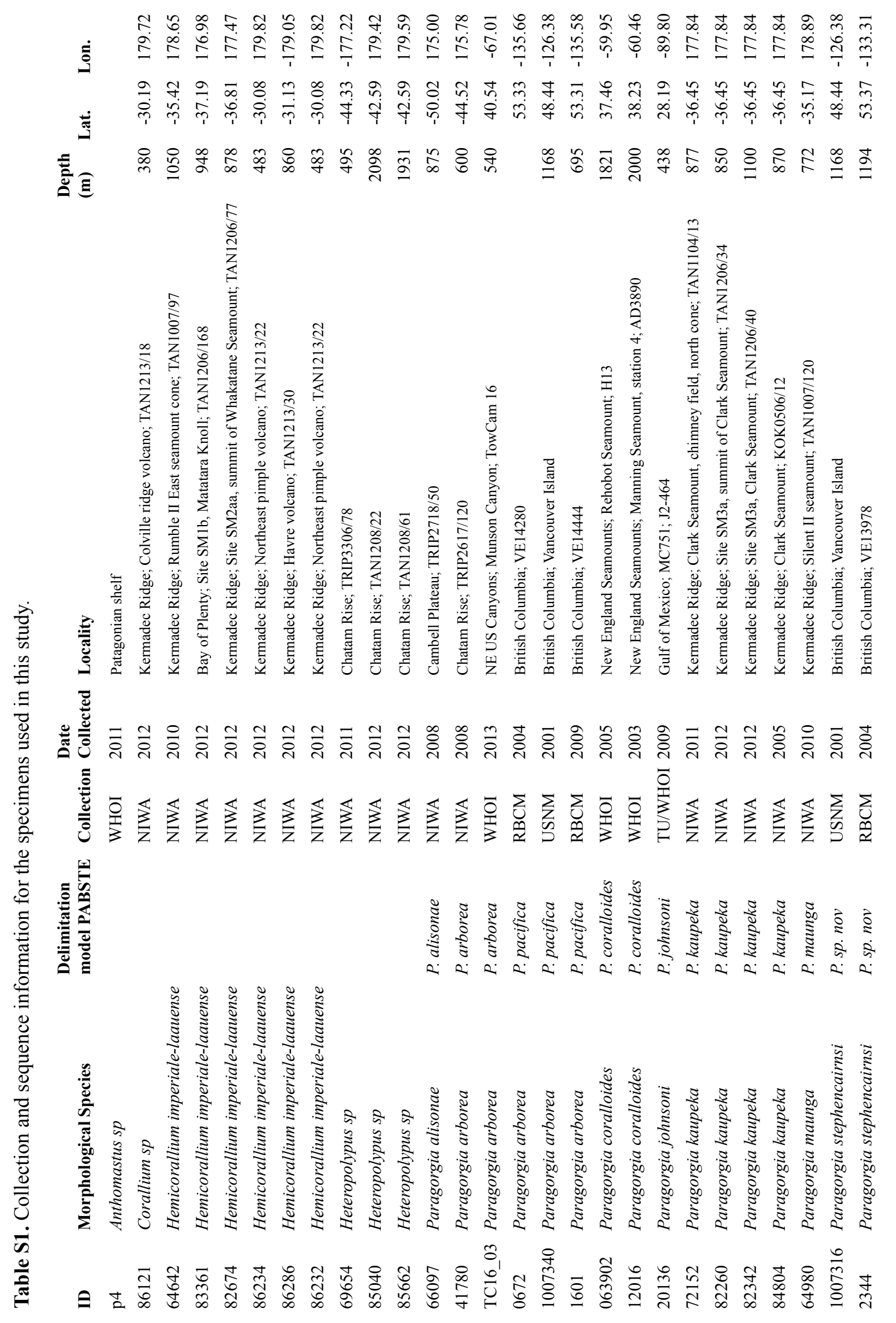


チ.

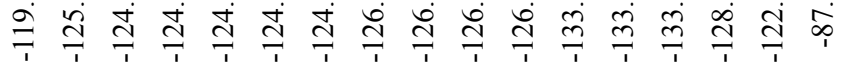

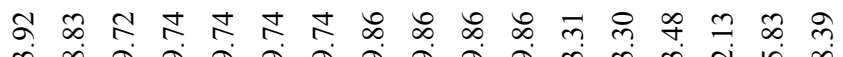

m

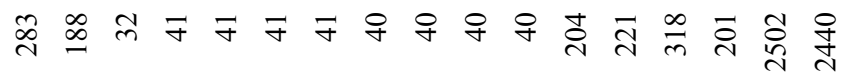

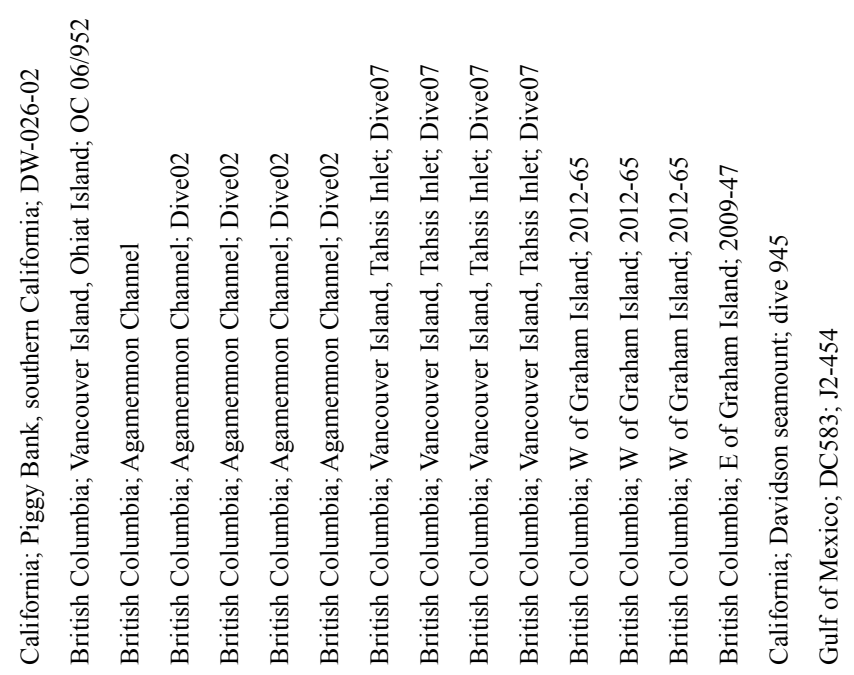

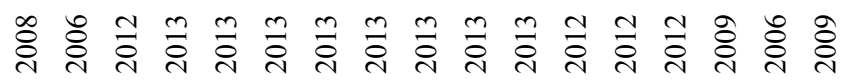

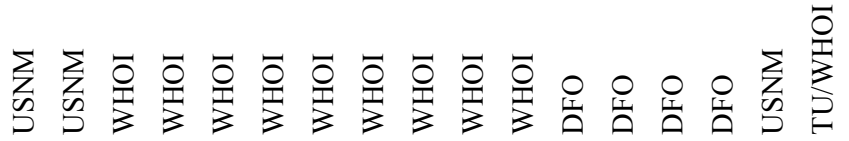

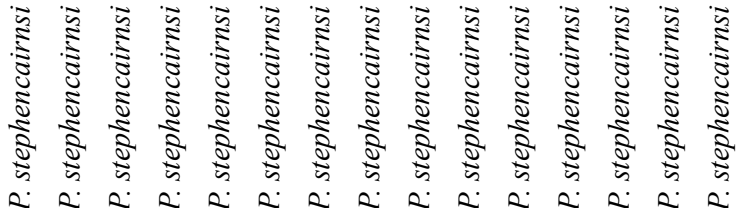

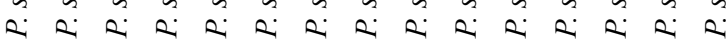

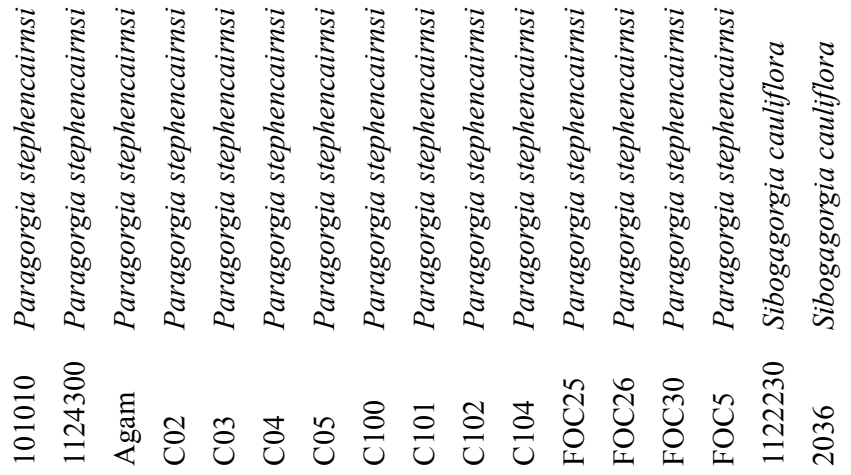


Table S2. RAD sequencing results and filtering statistics.

\begin{tabular}{|c|c|c|c|c|c|c|c|c|c|}
\hline \multirow[b]{2}{*}{$\begin{array}{l}\text { Morphological } \\
\text { Species }\end{array}$} & \multirow[b]{2}{*}{$\begin{array}{l}\text { Delimitation } \\
\text { model PABSTE }\end{array}$} & \multirow[b]{2}{*}{$\begin{array}{l}\text { RAD-seq data file } \\
\text { ID }\end{array}$} & \multirow[b]{2}{*}{$\begin{array}{l}\text { Total } \\
\text { sequenced } \\
\text { reads }\end{array}$} & \multicolumn{2}{|c|}{ STACKS filtering } & \multicolumn{3}{|c|}{ pyRAD filtering } & \multirow[b]{2}{*}{$\begin{array}{l}\text { \% of } \\
\text { retained } \\
\text { reads } \\
\text { after } \\
\text { filtering } \\
\text { steps }\end{array}$} \\
\hline & & & & $\begin{array}{l}\text { Reads } \\
\text { discarded } \\
\text { due to low } \\
\text { quality }\end{array}$ & $\begin{array}{l}\text { Retained } \\
\text { reads }\end{array}$ & $\begin{array}{l}\text { Reads } \\
\text { that } \\
\text { passed } \\
\text { quality } \\
\text { filtering }\end{array}$ & $\begin{array}{l}\text { Trimmed } \\
\text { reads due } \\
\text { to } \\
\text { detection } \\
\text { of } \\
\text { adapters }\end{array}$ & $\begin{array}{l}\text { Total } \\
\text { number } \\
\text { of } \\
\text { retained } \\
\text { reads }\end{array}$ & \\
\hline \multicolumn{2}{|l|}{ Anthomastus sp } & PoC_p4_ARG & $2,207,834$ & 151,391 & $1,312,903$ & $1,076,607$ & 38,570 & $1,115,177$ & 50.51 \\
\hline \multicolumn{2}{|l|}{ Corallium sp } & COR_86121_NZ & $3,826,317$ & 305,019 & $3,013,324$ & $2,555,644$ & 22,650 & $2,578,294$ & 67.38 \\
\hline \multicolumn{2}{|c|}{ H. imperiale-laauense } & COR_64642_NZ & $4,344,702$ & 239,625 & $4,105,077$ & $3,697,256$ & 21,823 & $3,719,079$ & 85.60 \\
\hline \multicolumn{2}{|c|}{ H. imperiale-laauense } & COR_83361_NZ & $3,351,944$ & 291,963 & $2,588,492$ & $2,103,039$ & 37,016 & $2,140,055$ & 63.85 \\
\hline \multicolumn{2}{|c|}{ H. imperiale-laauense } & HEM_82674_NZ & $4,455,288$ & 364,162 & $3,777,461$ & $3,216,577$ & 40,911 & $3,257,488$ & 73.12 \\
\hline \multicolumn{2}{|c|}{ H. imperiale-laauense } & HEM_86234_NZ & $2,408,325$ & 186,372 & $2,142,057$ & $1,876,187$ & 16,195 & $1,892,382$ & 78.58 \\
\hline \multicolumn{2}{|c|}{ H. imperiale-laauense } & HEM_86286_NZ & $5,324,532$ & 446,268 & $4,266,674$ & $3,577,980$ & 42,661 & $3,620,641$ & 68.00 \\
\hline \multicolumn{2}{|c|}{ H. imperiale-laauense } & PAR_86232_NZ & $6,092,276$ & 503,438 & $5,206,376$ & $4,454,042$ & 35,518 & $4,489,560$ & 73.69 \\
\hline \multicolumn{2}{|l|}{ Heteropolypus sp } & ANT_69654_NZ & $4,300,289$ & 349,226 & $3,645,814$ & $3,139,309$ & 32,395 & $3,171,704$ & 73.76 \\
\hline \multicolumn{2}{|l|}{ Heteropolypus sp } & ANT_85040_NZ & $4,498,336$ & 393,748 & $3,882,304$ & $3,333,172$ & 26,687 & $3,359,859$ & 74.69 \\
\hline \multicolumn{2}{|l|}{ Heteropolypus sp } & ANT_85662_NZ & $3,487,138$ & 283,856 & $2,812,750$ & $2,382,184$ & 40,135 & $2,422,319$ & 69.46 \\
\hline P. alisonae & P. alisonae & PAR_66097_NZ & $3,007,362$ & 220,209 & $2,511,717$ & $2,219,265$ & 21,207 & $2,240,472$ & 74.50 \\
\hline P. arborea & P. arborea & PAR_41780_NZ & $6,668,080$ & 367,144 & $6,300,936$ & $5,655,328$ & 33,758 & $5,689,086$ & 85.32 \\
\hline P. arborea & P. arborea & PAR_TC16_03_CAN & $2,259,880$ & 167,106 & $1,799,244$ & $1,556,363$ & 17,448 & $1,573,811$ & 69.64 \\
\hline P. arborea & P. pacifica & PAR_0672_BC & $4,348,226$ & 293,526 & $4,054,700$ & $3,543,642$ & 29,284 & $3,572,926$ & 82.17 \\
\hline P. arborea & P. pacifica & PAR_1007340_BCD & $2,808,882$ & 251,102 & $2,431,829$ & $2,063,940$ & 25,831 & $2,089,771$ & 74.40 \\
\hline P. arborea & P. pacifica & PAR_1601_BC & $4,202,185$ & 221,386 & $3,980,799$ & $3,606,334$ & 25,434 & $3,631,768$ & 86.43 \\
\hline P. coralloides & P. coralloides & PAR_063902_NES & $3,244,860$ & 185,122 & $3,059,738$ & $2,746,228$ & 20,408 & $2,766,636$ & 85.26 \\
\hline P. coralloides & P. coralloides & PAR_12016_NES & $3,838,375$ & 226,253 & $3,612,122$ & $3,255,440$ & 15,945 & $3,271,385$ & 85.23 \\
\hline P. johnsoni & P. johnsoni & PAR_20136_GOM & $7,751,624$ & 422,203 & $7,329,421$ & $6,623,446$ & 38,692 & $6,662,138$ & 85.95 \\
\hline P. kaupeka & P. kaupeka & PAR_72152_NZ & $3,909,139$ & 268,871 & $2,926,960$ & $2,531,796$ & 26,984 & $2,558,780$ & 65.46 \\
\hline P. kaupeka & P. kaupeka & PAR_82260_NZ & $5,511,873$ & 475,292 & $4,558,291$ & $3,824,683$ & 42,782 & $3,867,465$ & 70.17 \\
\hline P. kaupeka & P. kaupeka & PAR_82342_NZ & $4,056,319$ & 307,425 & $3,339,653$ & $2,866,786$ & 33,020 & $2,899,806$ & 71.49 \\
\hline P. kaupeka & P. kaupeka & PAR_84804_NZ & $2,828,879$ & 230,493 & $2,313,997$ & $1,968,495$ & 19,602 & $1,988,097$ & 70.28 \\
\hline P. maunga & P. maunga & PAR_11369_NZ & $2,536,311$ & 252,554 & $2,008,028$ & $1,619,915$ & 34,477 & $1,654,392$ & 65.23 \\
\hline P. stephencairnsi & P.sp.nov & PAR_1007316_BCD & $2,257,183$ & 151,729 & $1,606,180$ & $1,371,398$ & 36,692 & $1,408,090$ & 62.38 \\
\hline P. stephencairnsi & P. sp.nov & PAR_2344_BC & $2,858,311$ & 159,073 & $2,699,238$ & $2,414,787$ & 26,272 & $2,441,059$ & 85.40 \\
\hline P. stephencairnsi & P. stephencairnsi & PAR_101010_CA & $6,642,673$ & 398,317 & $6,244,356$ & $5,595,342$ & 39,240 & $5,634,582$ & 84.82 \\
\hline P. stephencairnsi & P. stephencairnsi & PAR_1124300_WA & $3,843,250$ & 215,859 & $3,627,391$ & $3,260,864$ & 27,921 & $3,288,785$ & 85.57 \\
\hline P. stephencairnsi & P. stephencairnsi & PAR_Agam_BC & $4,322,564$ & 260,095 & $4,062,469$ & $3,619,988$ & 26,669 & $3,646,657$ & 84.36 \\
\hline
\end{tabular}




\begin{tabular}{|c|c|c|c|c|c|c|c|c|c|}
\hline P. stephencairnsi & P. stephencairnsi & PAR_C02_BCS & $2,149,938$ & 153,978 & $1,594,818$ & $1,377,002$ & 16,157 & $1,393,159$ & 64.80 \\
\hline P. stephencairnsi & P. stephencairnsi & PAR_C03_BCS & $2,815,327$ & 245,051 & $2,264,951$ & $1,916,452$ & 19,800 & $1,936,252$ & 68.78 \\
\hline P. stephencairnsi & P. stephencairnsi & PAR_C04_BCS & $3,240,713$ & 267,042 & $2,754,373$ & $2,379,284$ & 22,261 & $2,401,545$ & 74.11 \\
\hline P. stephencairnsi & P. stephencairnsi & PAR_C05_BCS & $3,465,396$ & 294,547 & $2,972,548$ & $2,538,577$ & 25,005 & $2,563,582$ & 73.98 \\
\hline P. stephencairnsi & P. stephencairnsi & PAR_C100_BCS & $5,998,914$ & 480,465 & $4,984,766$ & $4,330,930$ & 38,445 & $4,369,375$ & 72.84 \\
\hline P. stephencairnsi & P. stephencairnsi & PAR_C101_BCS & $5,332,619$ & 446,452 & $4,652,535$ & $4,030,919$ & 30,684 & $4,061,603$ & 76.17 \\
\hline P. stephencairnsi & P. stephencairnsi & PAR_C102_BCS & $4,347,757$ & 348,662 & $3,710,381$ & $3,221,934$ & 26,559 & $3,248,493$ & 74.72 \\
\hline P. stephencairnsi & P. stephencairnsi & PAR_C104_BCS & $2,720,994$ & 210,479 & $2,203,568$ & $1,893,649$ & 19,213 & $1,912,862$ & 70.30 \\
\hline P. stephencairnsi & P. stephencairnsi & PAR_FOC25_BCD & $5,479,109$ & 453,625 & $4,693,804$ & $4,073,503$ & 34,583 & $4,108,086$ & 74.98 \\
\hline P. stephencairnsi & P. stephencairnsi & PAR_FOC26_BCD & $5,242,949$ & 477,361 & $4,528,629$ & $3,778,655$ & 37,137 & $3,815,792$ & 72.78 \\
\hline P. stephencairnsi & P. stephencairnsi & PAR_FOC30_BCD & $4,417,520$ & 337,501 & $3,781,973$ & $3,312,791$ & 32,621 & $3,345,412$ & 75.73 \\
\hline P. stephencairnsi & P. stephencairnsi & PAR_FOC5_BCD & $2,834,377$ & 239,051 & $2,455,041$ & $2,109,251$ & 23,444 & $2,132,695$ & 75.24 \\
\hline S. cauliflora & & SIB_1122230_DAV & $1,701,751$ & 123,755 & $1,220,077$ & $1,016,203$ & 26,104 & $1,042,307$ & 61.25 \\
\hline \multirow[t]{3}{*}{ S. cauliflora } & & SIB_2036_GOM & $2,638,533$ & 191,392 & $2,373,232$ & $2,077,092$ & 20,727 & $2,097,819$ & 79.51 \\
\hline & & AVERAGE & $3,944,974$ & 292,232 & $3,395,704$ & $2,950,279$ & 28,840 & $2,979,119$ & 74.27 \\
\hline & & STD DEV. & $1,392,573$ & 105,332 & $1,352,276$ & $1,219,146$ & 7,968 & $1,222,799$ & 8.09 \\
\hline
\end{tabular}




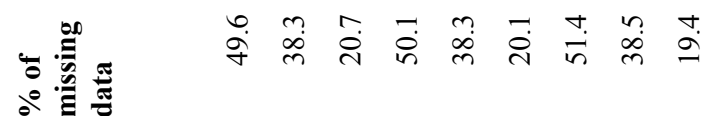

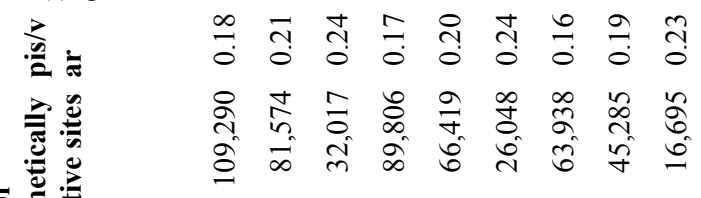

\section{\#}

跑高高高

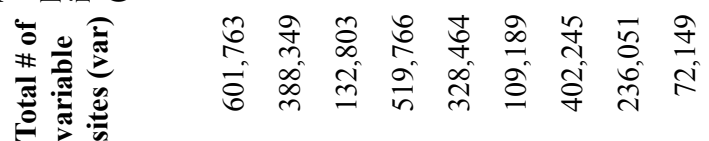

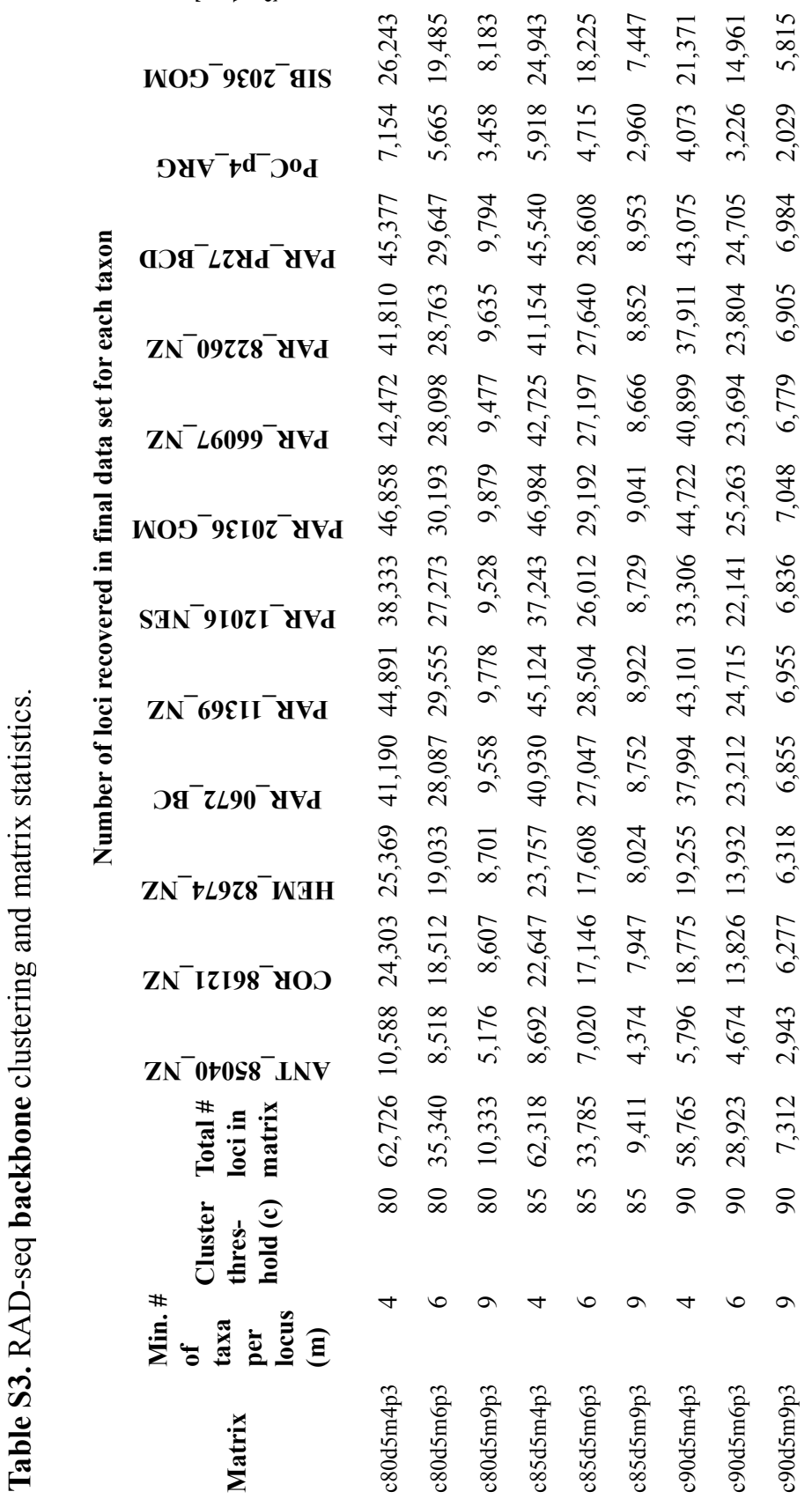




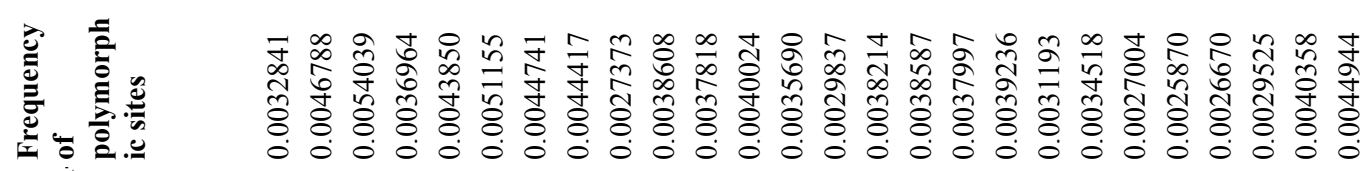

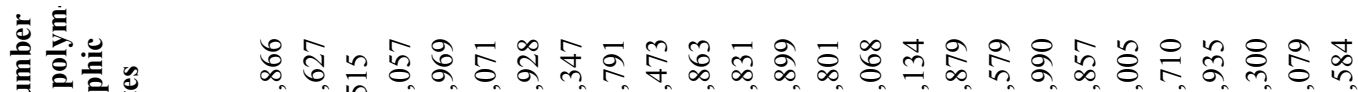
Wh IL

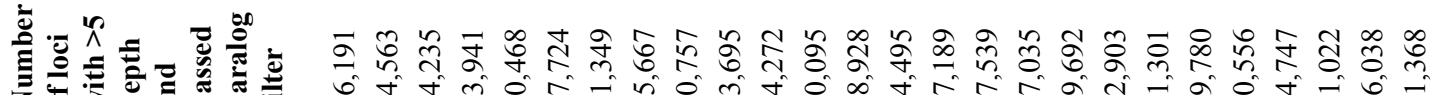

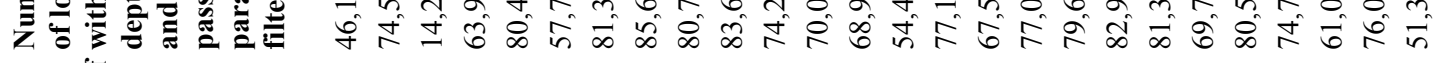
-

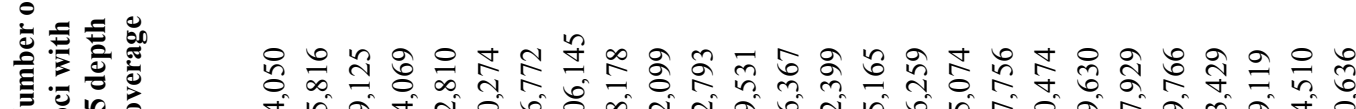

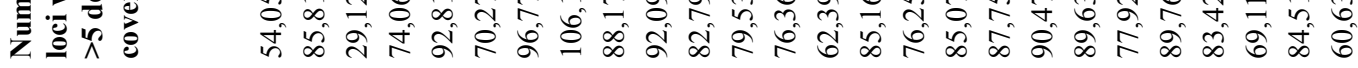

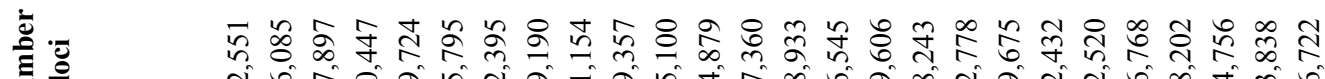

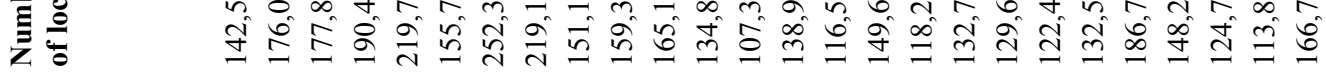

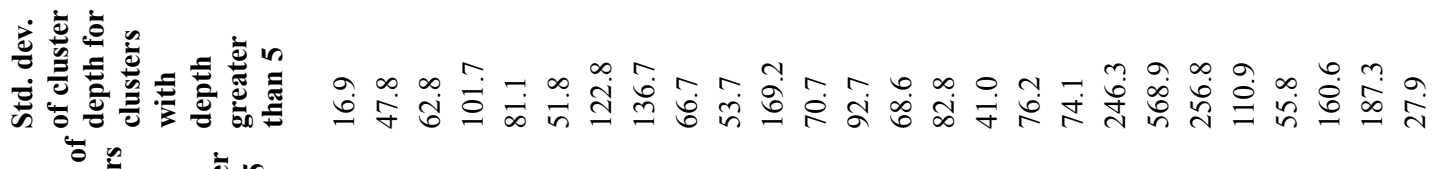

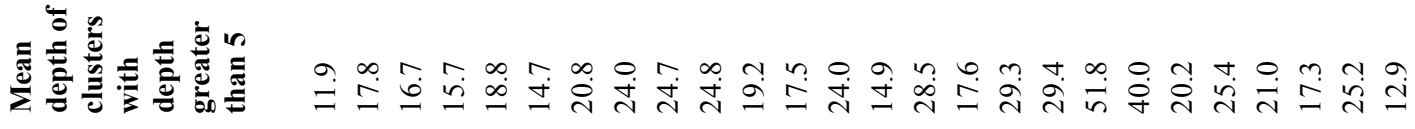

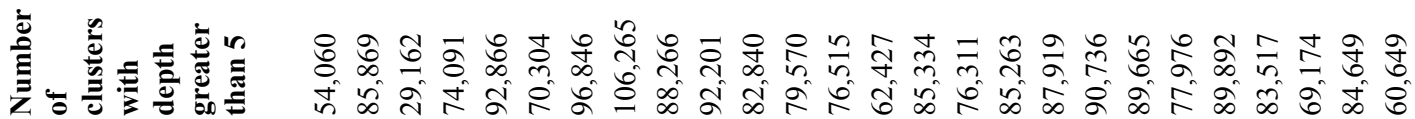

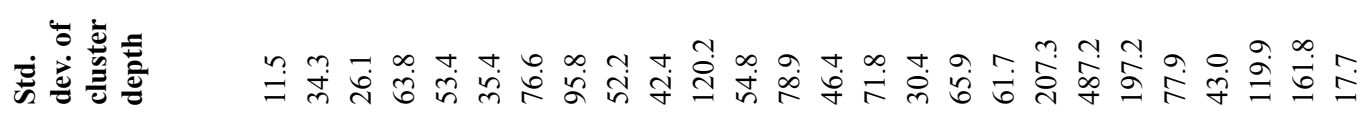

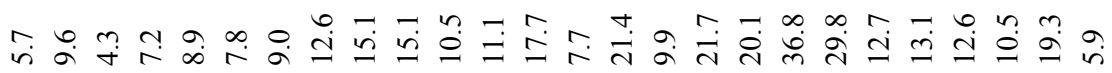

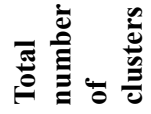

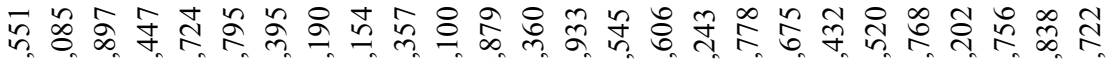

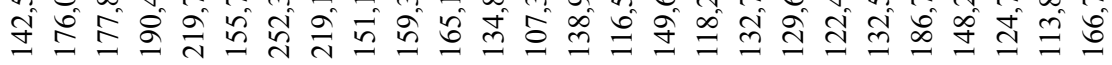

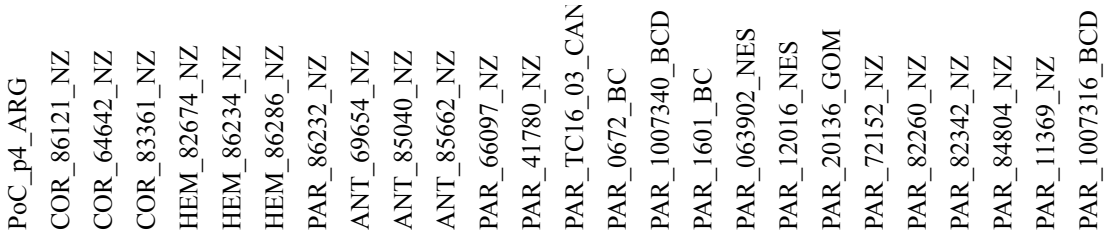
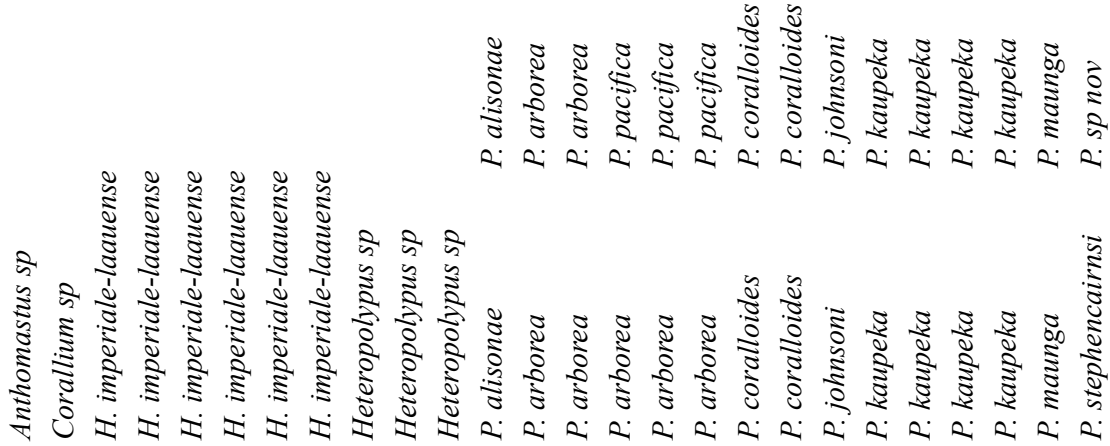


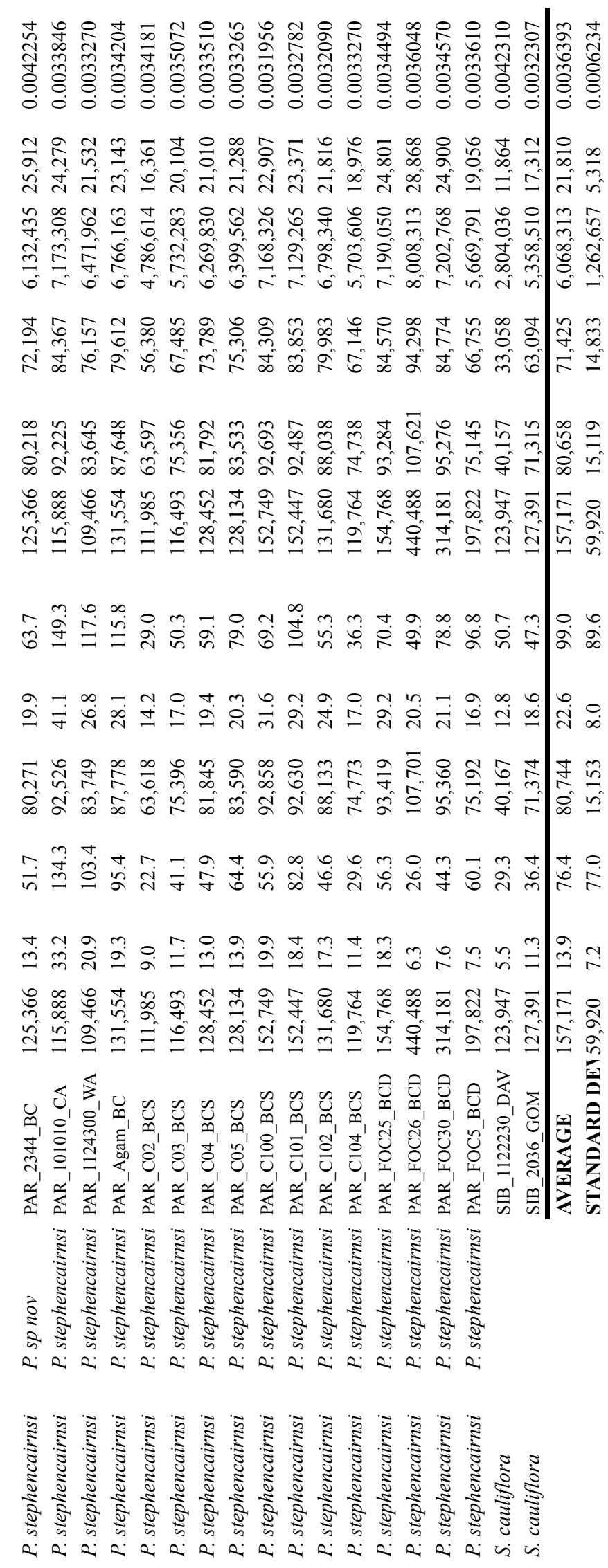


Table S5. Nucleotide diversity and error rate estimates per specimen based on the PHYLO matrix

\begin{tabular}{|c|c|}
\hline Morphological Species & PABSTI \\
\hline \multicolumn{2}{|l|}{ Anthomastus sp } \\
\hline \multicolumn{2}{|l|}{ Corallium sp } \\
\hline \multicolumn{2}{|l|}{ H. imperiale-laauense* } \\
\hline \multicolumn{2}{|l|}{ H. imperiale-laauense } \\
\hline \multicolumn{2}{|l|}{ H. imperiale-laauense } \\
\hline \multicolumn{2}{|l|}{ H. imperiale-laauense } \\
\hline \multicolumn{2}{|l|}{ H. imperiale-laauense } \\
\hline \multicolumn{2}{|l|}{ H. imperiale-laauense } \\
\hline \multicolumn{2}{|l|}{ Heteropolypus $s p$} \\
\hline \multicolumn{2}{|l|}{ Heteropolypus sp } \\
\hline \multicolumn{2}{|l|}{ Heteropolypus $s p$} \\
\hline P. alisonae & P. alisonae \\
\hline P. arborea & P. arborea \\
\hline P. arborea & P. arborea \\
\hline P. arborea & P. pacifica \\
\hline P. arborea & P. pacifica \\
\hline P. arborea & P. pacifica \\
\hline P. coralloides & P. coralloides \\
\hline P. coralloides & P. coralloides \\
\hline P. johnsoni & P. johnsoni \\
\hline P. kaupeka & P. kaupeka \\
\hline P. kaupeka & P. kaupeka \\
\hline P. kaupeka & P. kaupeka \\
\hline P. kaupeka & P. kaupeka \\
\hline P. maunga & P. maunga \\
\hline P. stephencairnsi & P.sp.nov \\
\hline P. stephencairnsi & P.sp.nov \\
\hline P. stephencairnsi & P. stephencairnsi \\
\hline P. stephencairnsi & P. stephencairnsi \\
\hline P. stephencairnsi & P. stephencairnsi \\
\hline P. stephencairnsi & P. stephencairnsi \\
\hline P. stephencairnsi & P. stephencairnsi \\
\hline P. stephencairnsi & P. stephencairnsi \\
\hline P. stephencairnsi & P. stephencairnsi \\
\hline P. stephencairnsi & P. stephencairnsi \\
\hline P. stephencairnsi & P. stephencairnsi \\
\hline P. stephencairnsi & P. stephencairnsi \\
\hline P. stephencairnsi & P. stephencairnsi \\
\hline P. stephencairnsi & P. stephencairnsi \\
\hline P. stephencairnsi & P. stephencairnsi \\
\hline P. stephencairnsi & P. stephencairnsi \\
\hline P. stephencairnsi & P. stephencairnsi \\
\hline S. cauliflora & \\
\hline S. cauliflora & \\
\hline
\end{tabular}

\begin{tabular}{|c|c|c|}
\hline RAD-seq data file ID & Nucleotide diversity (Pi) & Error Rate \\
\hline PoC_p4_ARG & 0.01295412 & 0.0029032 \\
\hline COR_86121_NZ & 0.01467291 & 0.00258843 \\
\hline COR_64642_NZ & 0.04406216 & 0.01630744 \\
\hline COR_83361_NZ & 0.01399387 & 0.00269528 \\
\hline HEM_82674_NZ & 0.01440962 & 0.00268885 \\
\hline HEM_86234_NZ & 0.01676557 & 0.00254558 \\
\hline HEM_86286_NZ & 0.01526906 & 0.00234296 \\
\hline PAR_86232_NZ & 0.01633107 & 0.00217237 \\
\hline ANT_69654_NZ & 0.00936378 & 0.00151989 \\
\hline ANT_85040_NZ & 0.01122064 & 0.00146665 \\
\hline ANT_85662_NZ & 0.01161827 & 0.00189506 \\
\hline PAR_66097_NZ & 0.0127996 & 0.00205016 \\
\hline PAR_41780_NZ & 0.01209999 & 0.00186767 \\
\hline PAR_TC16_03_CAN & 0.01293451 & 0.00241858 \\
\hline PAR_0672_BC & 0.01189447 & 0.00170062 \\
\hline PAR_1007340_BCD & 0.01285038 & 0.00223148 \\
\hline PAR_1601_BC & 0.01205019 & 0.00164477 \\
\hline PAR_063902_NES & 0.01182997 & 0.00146894 \\
\hline PAR_12016_NES & 0.01035726 & 0.00116583 \\
\hline PAR_20136_GOM & 0.01159218 & 0.00137491 \\
\hline PAR_72152_NZ & 0.01085919 & 0.00209157 \\
\hline PAR_82260_NZ & 0.01077562 & 0.00211998 \\
\hline PAR_82342_NZ & 0.01082052 & 0.00225821 \\
\hline PAR_84804_NZ & 0.01169178 & 0.00221668 \\
\hline PAR_11369_NZ & 0.01225252 & 0.00176518 \\
\hline PAR_1007316_BCD & 0.01488811 & 0.00295065 \\
\hline PAR_2344_BC & 0.01260347 & 0.00188425 \\
\hline PAR_101010_CA & 0.01063818 & 0.00134692 \\
\hline PAR_1124300_WA & 0.01092481 & 0.00163497 \\
\hline PAR_Agam_BC & 0.01117934 & 0.00151902 \\
\hline PAR_C02_BCS & 0.01180863 & 0.00194975 \\
\hline PAR_C03_BCS & 0.01129071 & 0.00175198 \\
\hline PAR_C04_BCS & 0.01098867 & 0.00171446 \\
\hline PAR_C05_BCS & 0.01117442 & 0.00167389 \\
\hline PAR_C100_BCS & 0.01063313 & 0.00155401 \\
\hline PAR_C101_BCS & 0.0109634 & 0.00160305 \\
\hline PAR_C102_BCS & 0.01060609 & 0.00159815 \\
\hline PAR_C104_BCS & 0.01124495 & 0.00175065 \\
\hline PAR_FOC25_BCD & 0.01104859 & 0.00154584 \\
\hline PAR_FOC26_BCD & 0.01263196 & 0.00180015 \\
\hline PAR_FOC30_BCD & 0.01196811 & 0.00182162 \\
\hline PAR_FOC5_BCD & 0.0119129 & 0.00186229 \\
\hline SIB_1122230_DAV & 0.01525699 & 0.00328691 \\
\hline SIB_2036_GOM & 0.01214983 & 0.00221517 \\
\hline AVERAGE & 0.01291599 & 0.00228532 \\
\hline SD & 0.00513429 & 0.00223806 \\
\hline
\end{tabular}

*Excluded from calculations due to low number of loci 
Table S6. Nucleotide diversity and error rate estimates per species based on the PHYLO matrix

\begin{tabular}{|c|c|c|c|c|c|c|c|}
\hline \multirow[b]{2}{*}{$\begin{array}{l}\text { Morphological } \\
\text { Species }\end{array}$} & \multirow[b]{2}{*}{$\begin{array}{l}\text { Delimitation } \\
\text { model PABSTE }\end{array}$} & \multicolumn{3}{|c|}{ Nucleeotide diversity (Pi) } & \multicolumn{3}{|c|}{ Per-site sequence error rate (Epsilon) } \\
\hline & & mean & SD & SE & mean & SD & SE \\
\hline Anthomastus sp & & 0.01295412 & & & 0.00290320 & & \\
\hline Corallium $s p$ & & 0.01467291 & & & 0.00258843 & & \\
\hline H. imperiale-laauense & & 0.01935775 & 0.01094005 & 0.00489254 & 0.00447727 & 0.00522005 & 0.00233448 \\
\hline Heteropolypus sp & & 0.01073423 & 0.00120338 & 0.00069477 & 0.00162720 & 0.00023350 & 0.00013481 \\
\hline P. alisonae & P. alisonae & 0.01279960 & & & 0.00205016 & & \\
\hline P. arborea & P. arborea & 0.01245029 & 0.00056582 & 0.00040010 & 0.00193813 & 0.00041487 & 0.00029336 \\
\hline P. arborea & P. pacifica & 0.01073423 & 0.00120338 & 0.00069477 & 0.00162720 & 0.00023350 & 0.00013481 \\
\hline P. coralloides & P. coralloides & 0.01245029 & 0.00056582 & 0.00040010 & 0.00193813 & 0.00041487 & 0.00029336 \\
\hline P. johnsoni & P. johnsoni & 0.01159218 & & & 0.00137491 & & \\
\hline P. kaupeka & P. kaupeka & 0.01241323 & 0.00175196 & 0.00087598 & 0.00229768 & 0.00048923 & 0.00024462 \\
\hline P. maunga & P. maunga & 0.01225252 & & & 0.00176518 & & \\
\hline P. stephencairnsi & P.sp.nov & 0.01290646 & 0.00280248 & 0.00198165 & 0.00229281 & 0.00093033 & 0.00065784 \\
\hline P. stephencairnsi & P. stephencairnsi & 0.01137950 & 0.00066172 & 0.00016543 & 0.00169174 & 0.00016364 & 0.00004091 \\
\hline S. cauliflora & & 0.01370341 & 0.00219709 & 0.00155358 & 0.00275104 & 0.00075783 & 0.00053587 \\
\hline
\end{tabular}




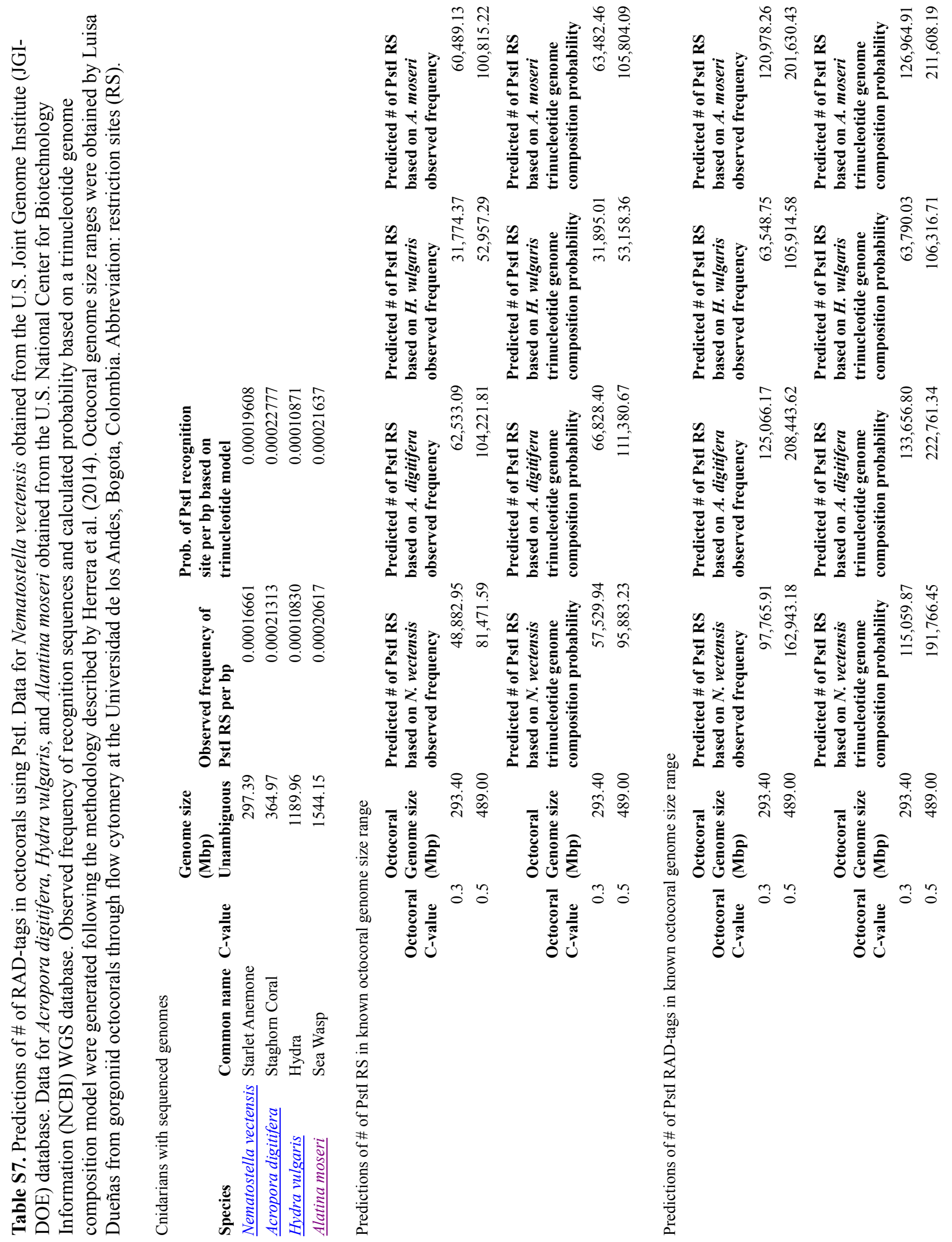




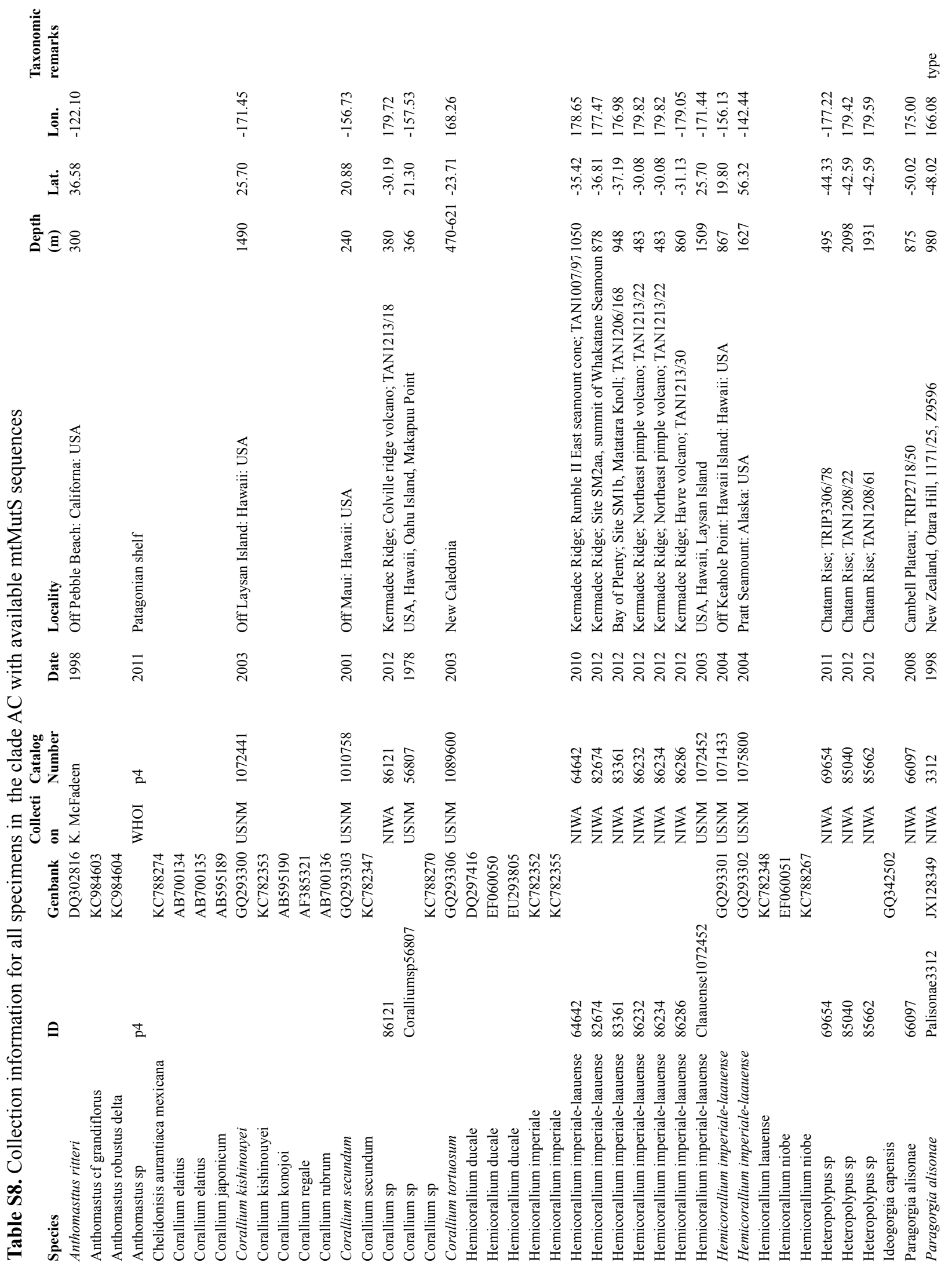




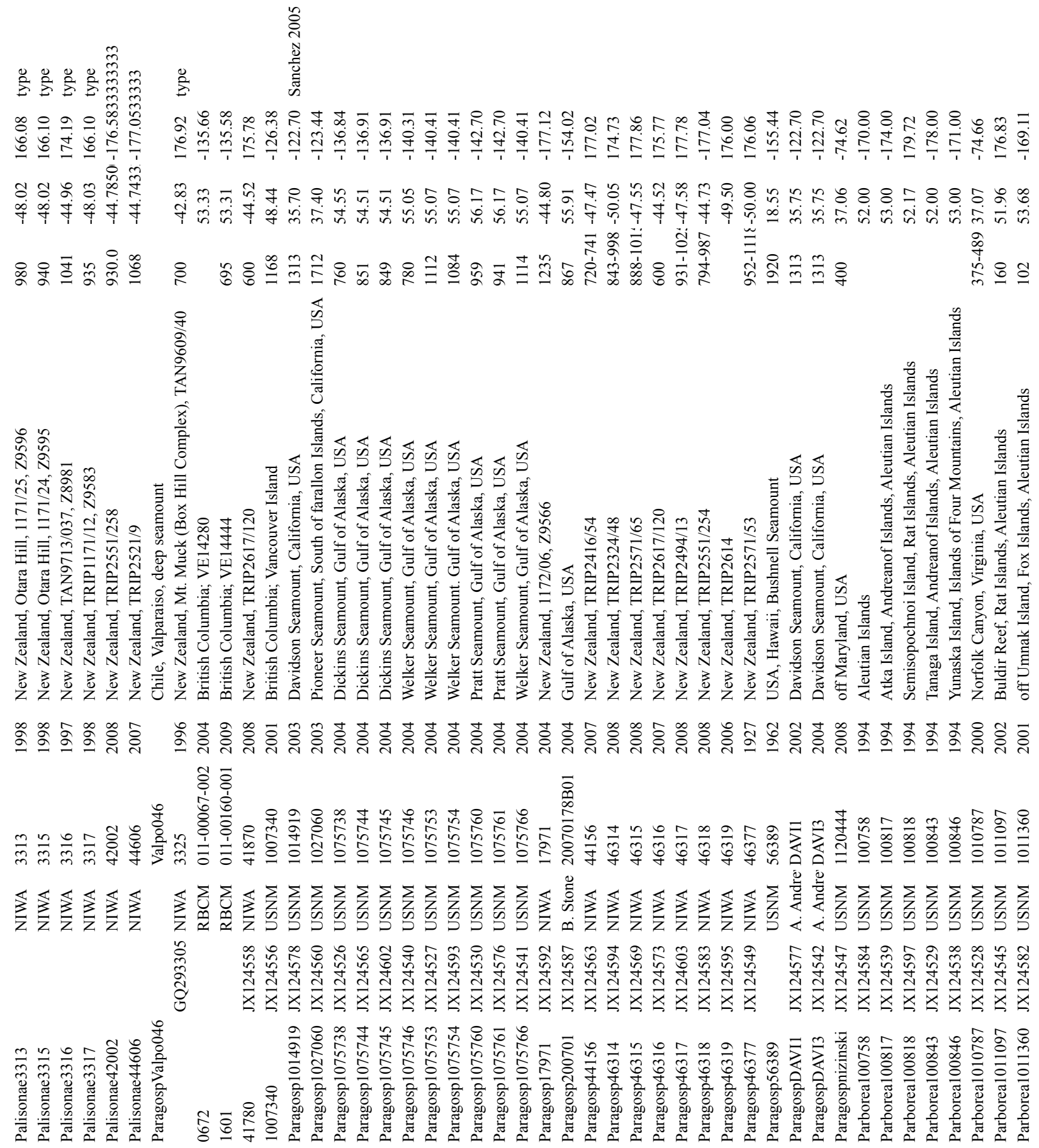

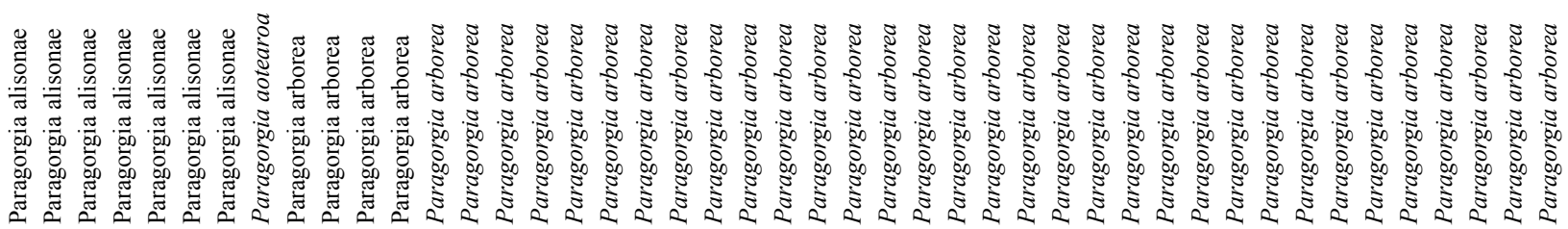




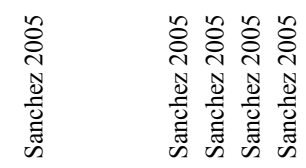

운

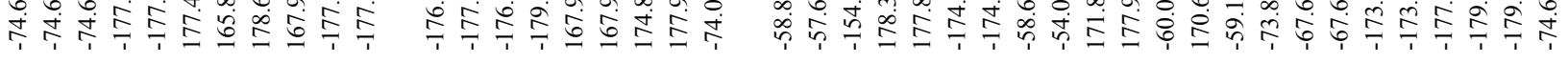

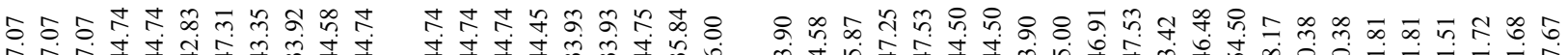

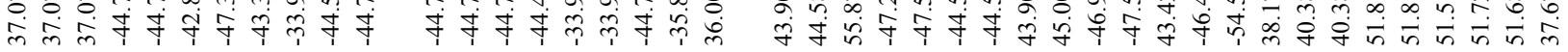

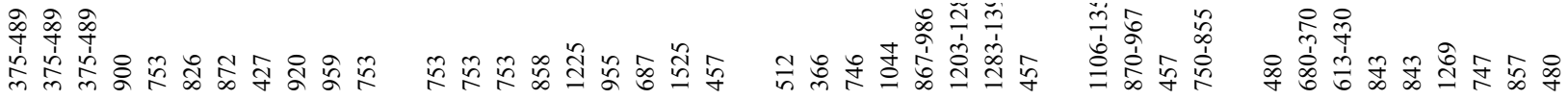

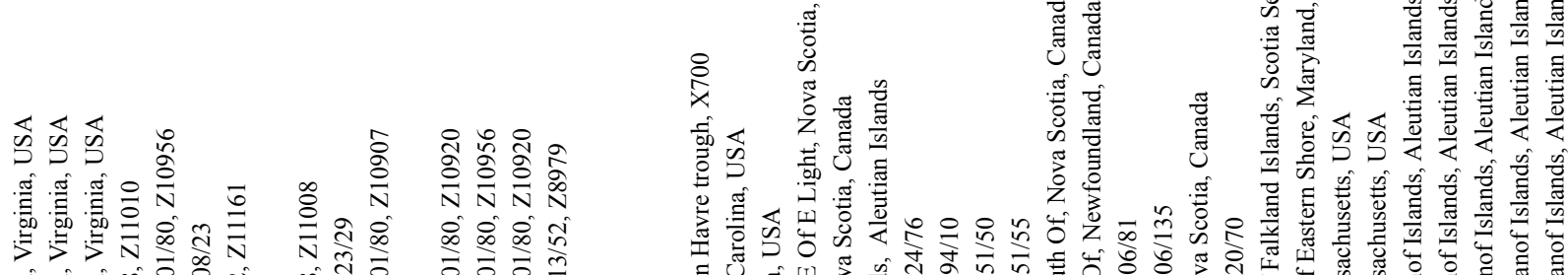

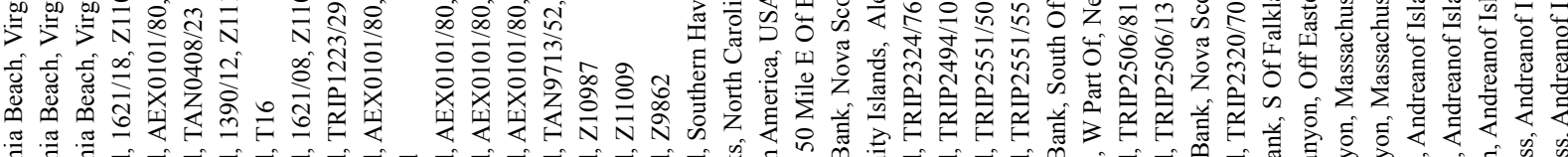

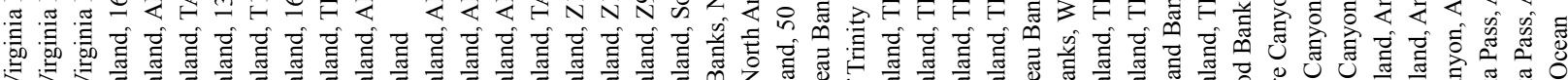

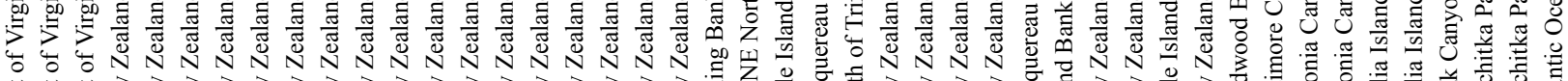

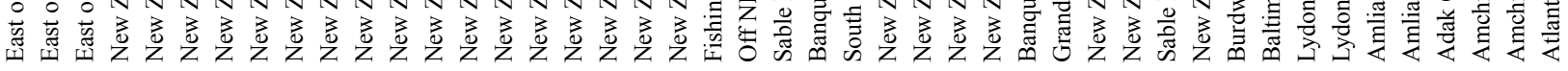

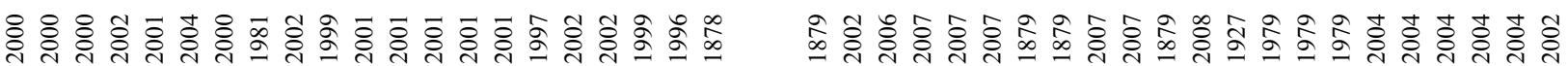

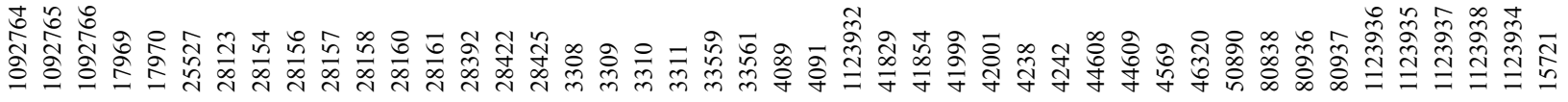

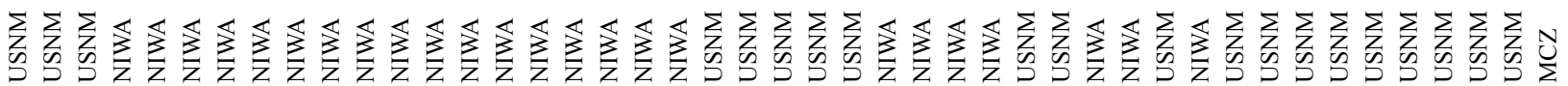

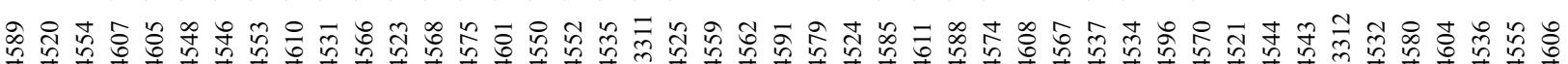

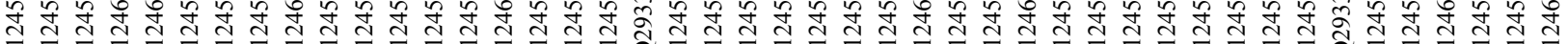

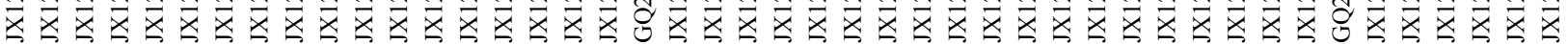

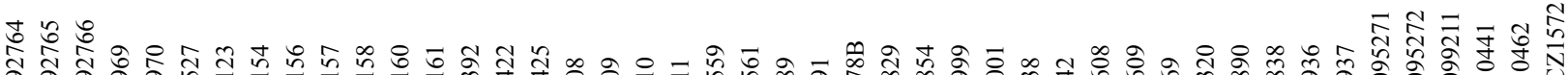

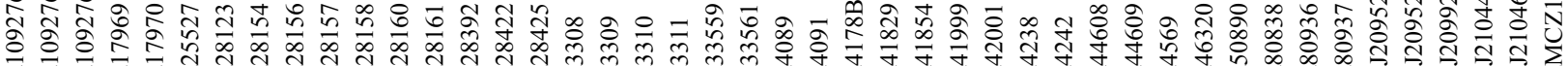

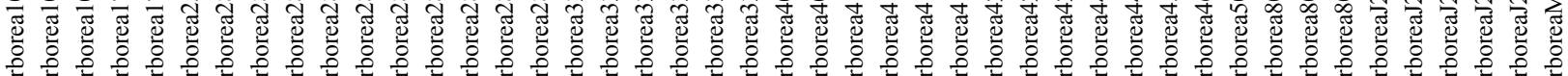

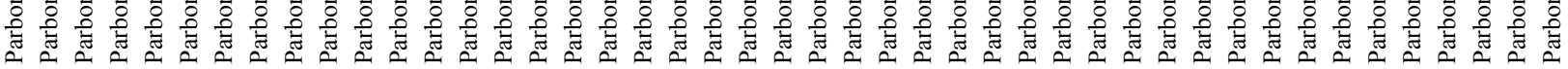

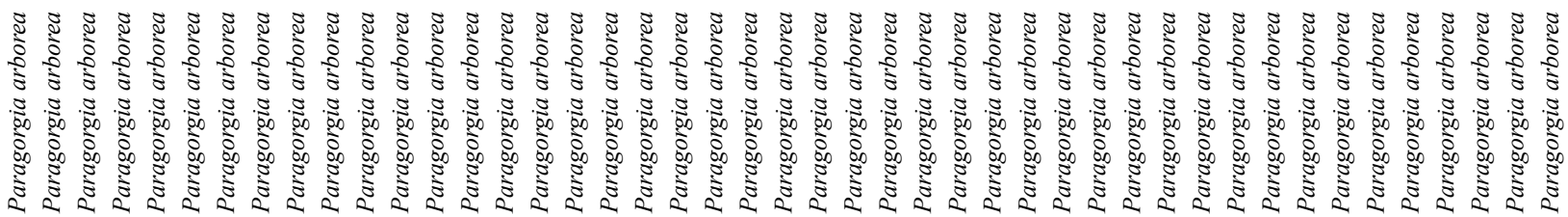




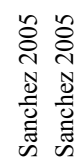

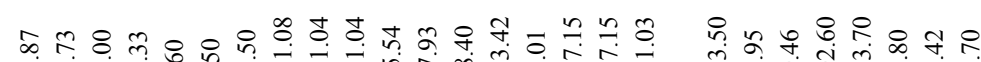

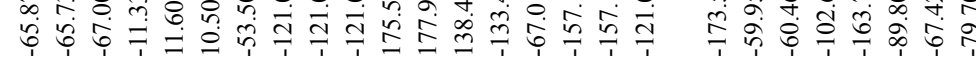

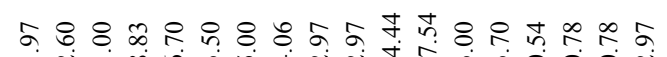

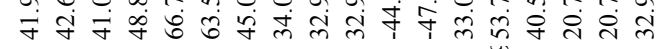

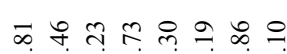

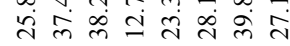

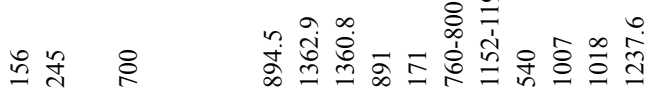

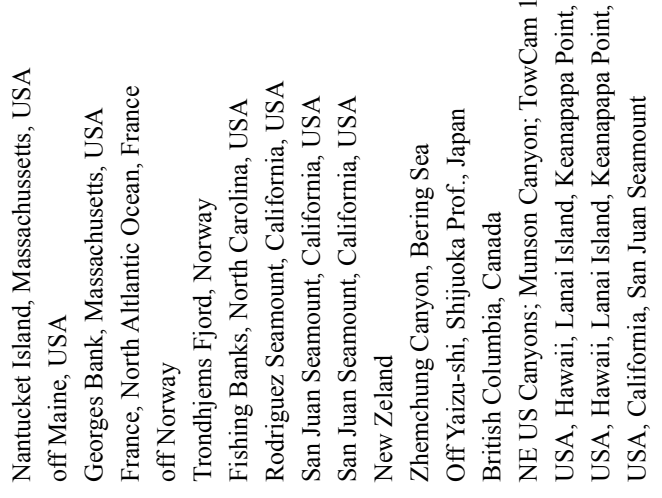

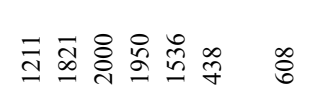

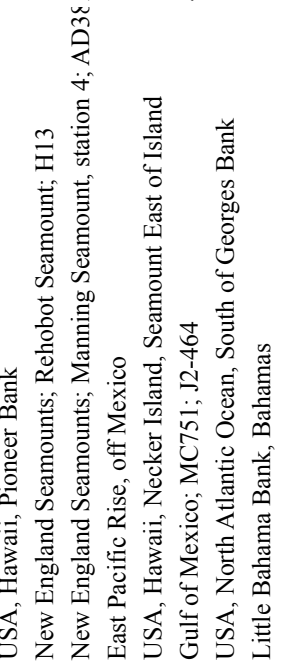

\section{总产产}

स

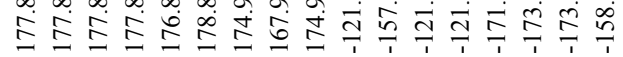

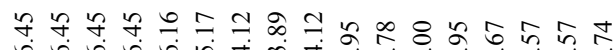

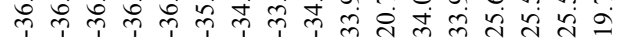

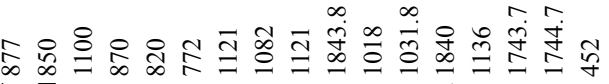

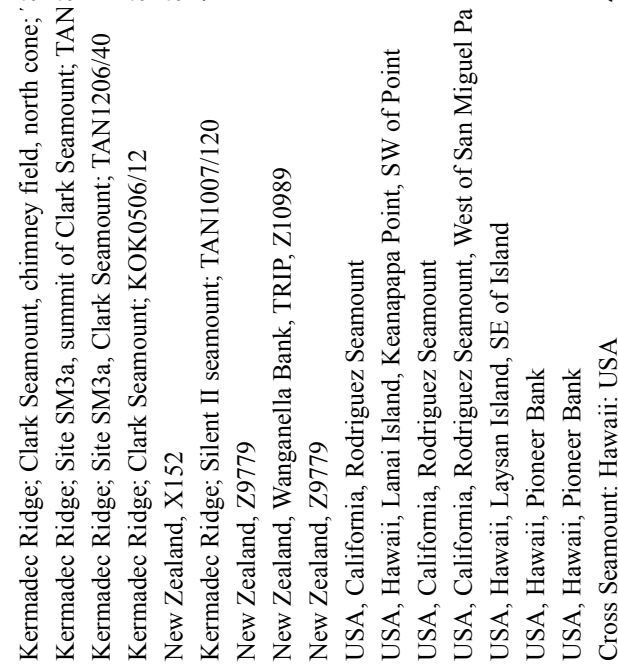

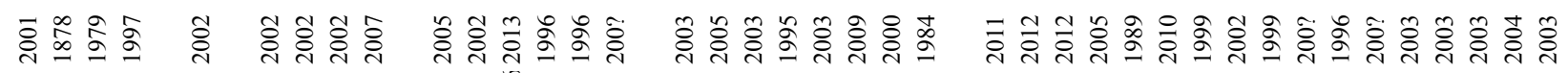

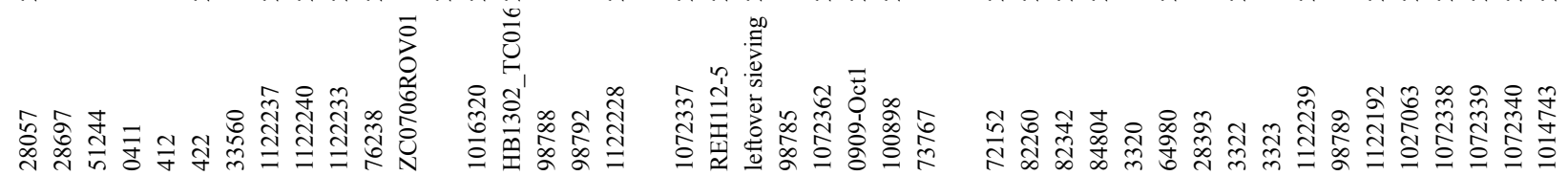

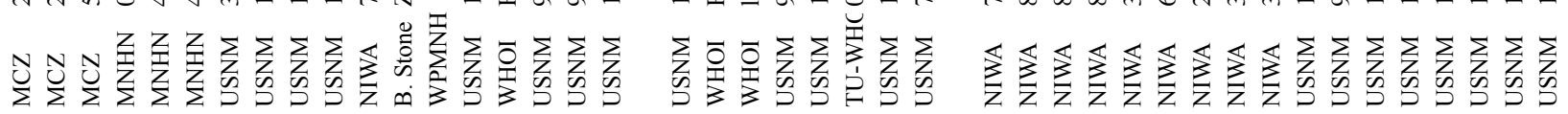

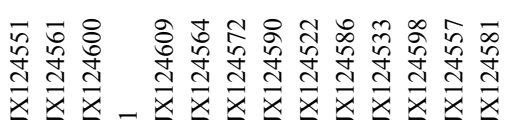

Whing

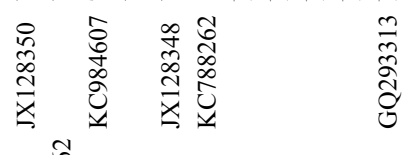
商

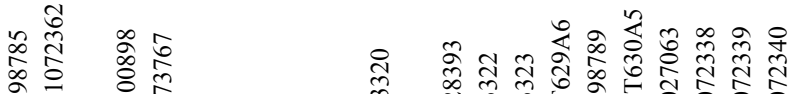

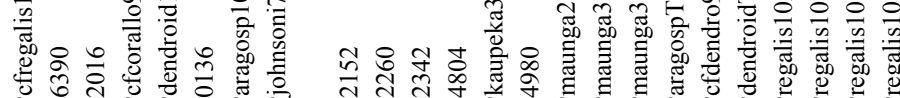
111

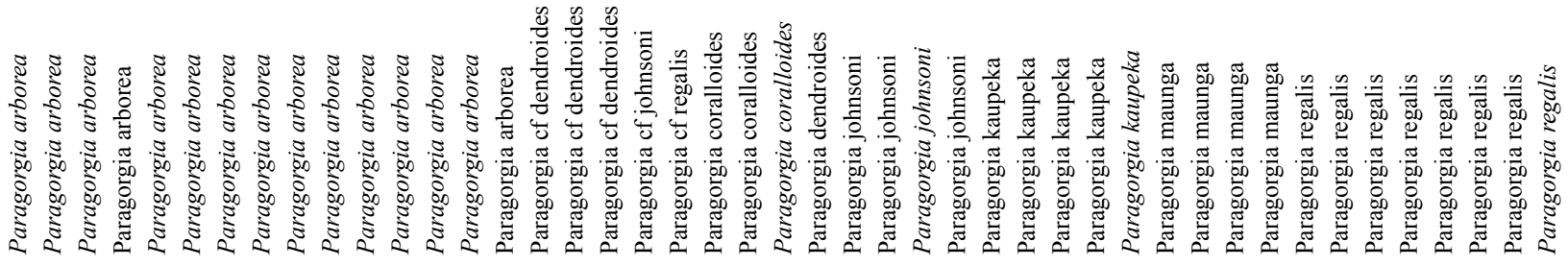




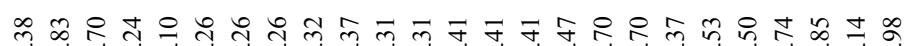

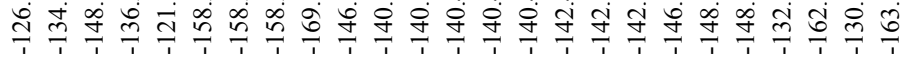

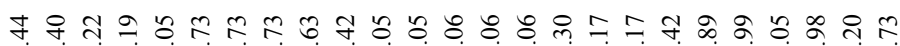

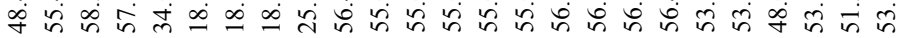
$\infty_{\infty}^{\infty} \infty_{\infty}^{\infty} i_{i}$

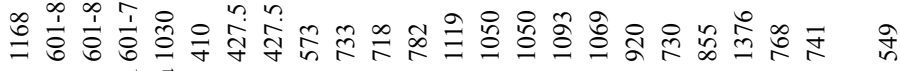
可

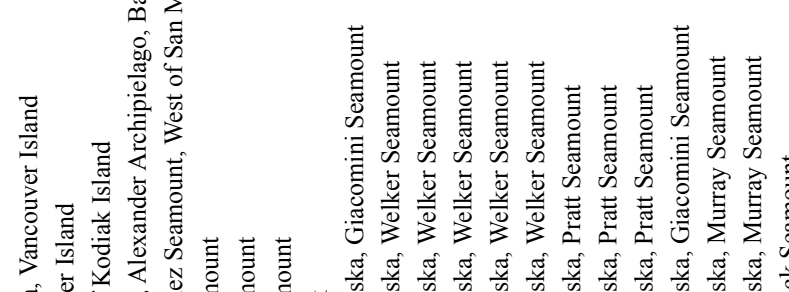
1 minn

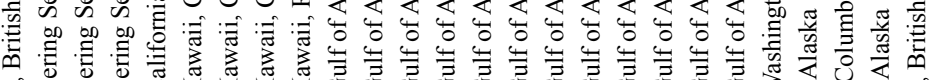

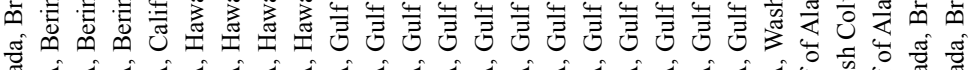

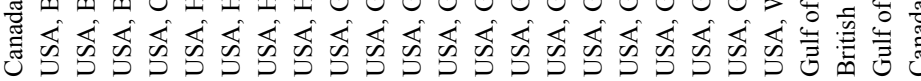

赤电的的 फ़ن

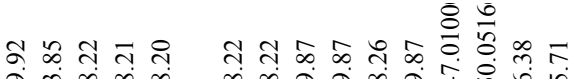

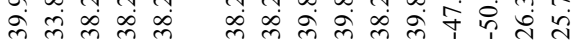

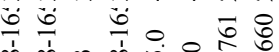

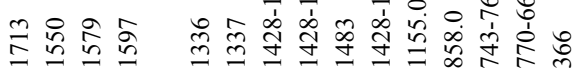

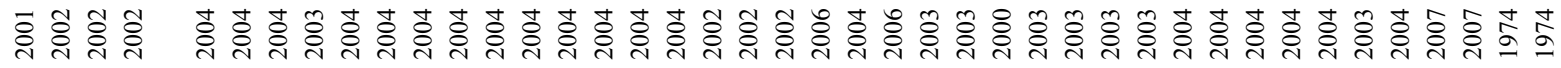

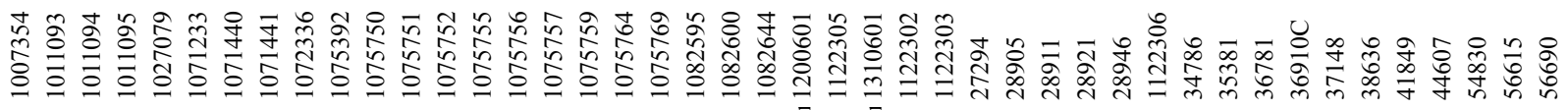

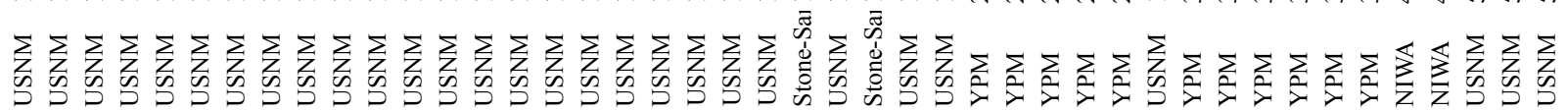

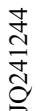

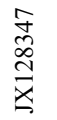

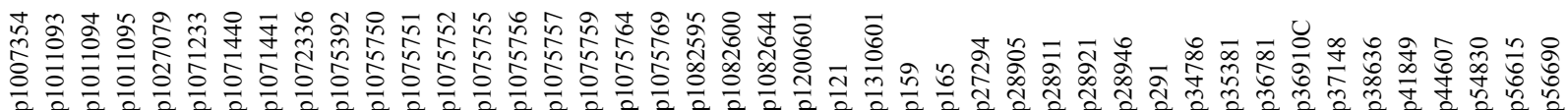

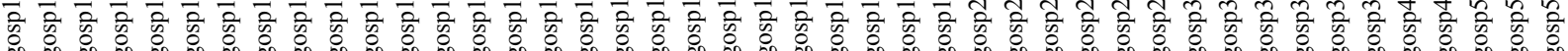

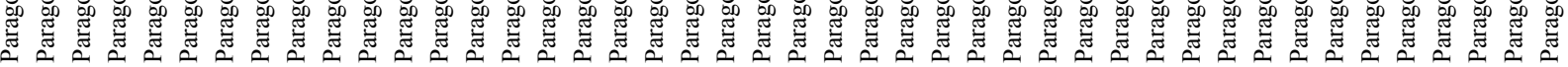

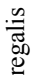

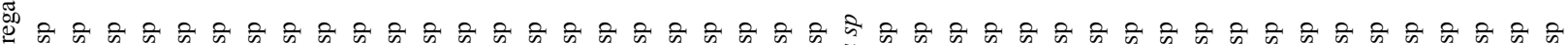

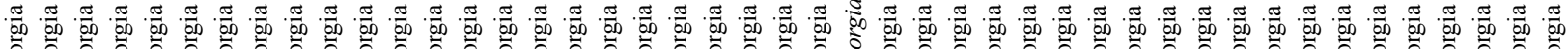

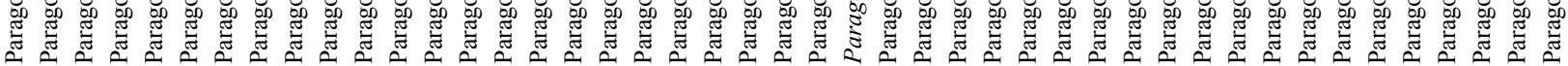


䒘

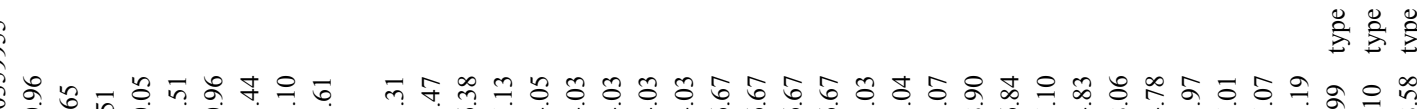

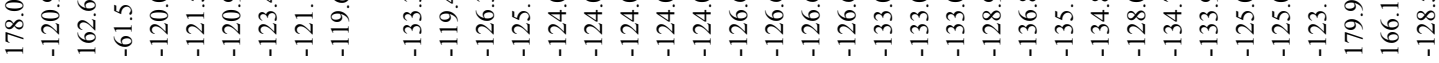

范

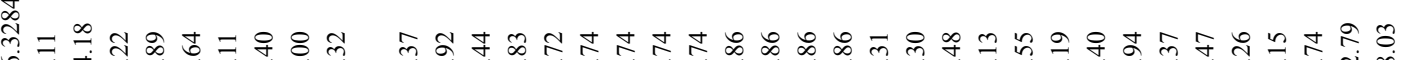

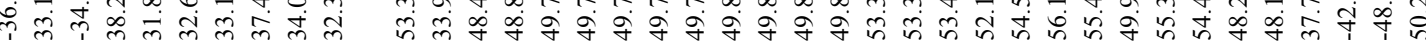

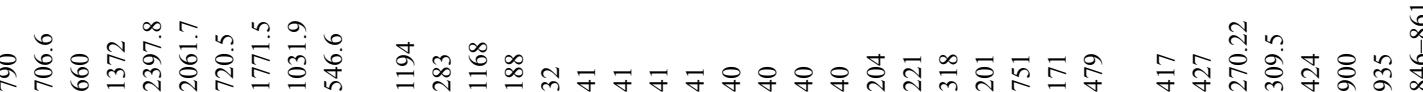
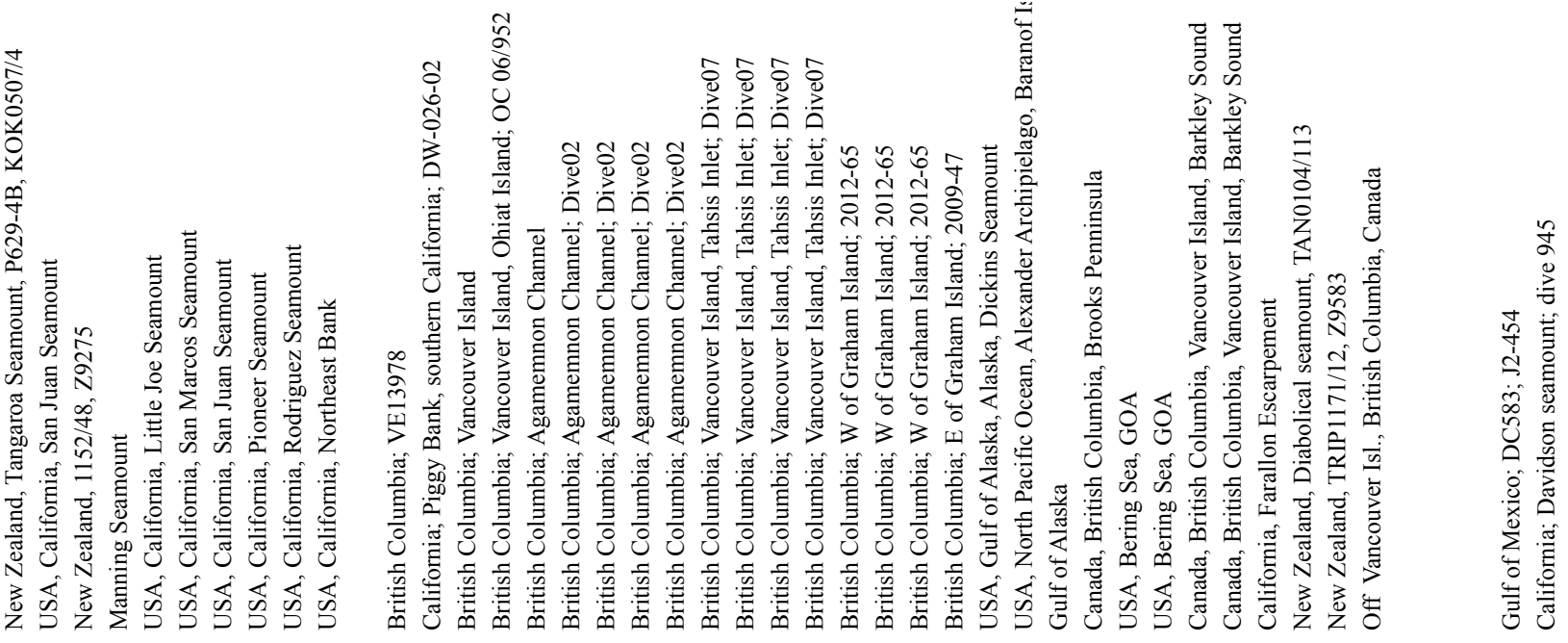

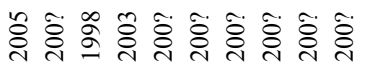

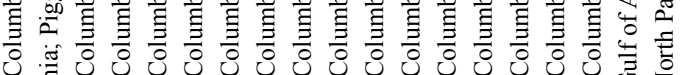

క్.

o

के तै

ले $\infty$

尔路

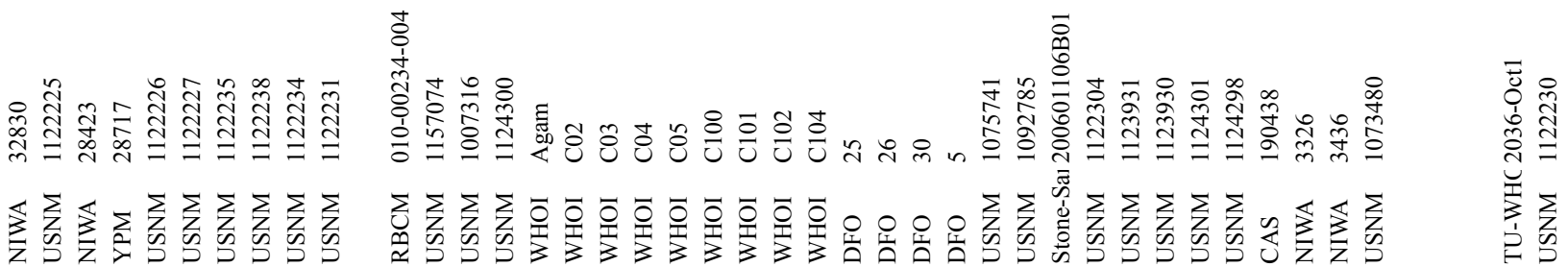

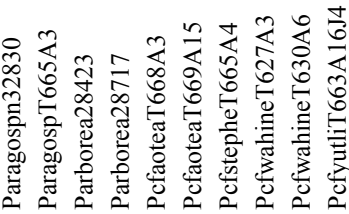

$$
\begin{aligned}
& \overrightarrow{0} \\
& 0 \\
& \infty \\
& 0 \\
& 0 \\
& 0 \\
& 0
\end{aligned}
$$

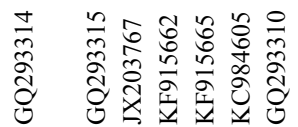

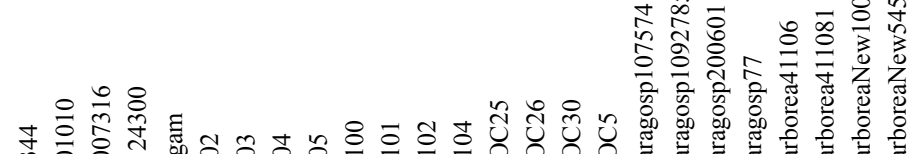
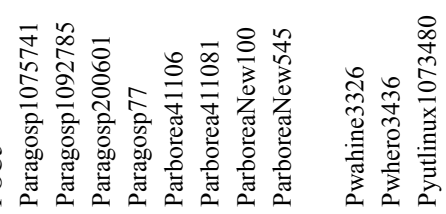

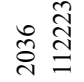

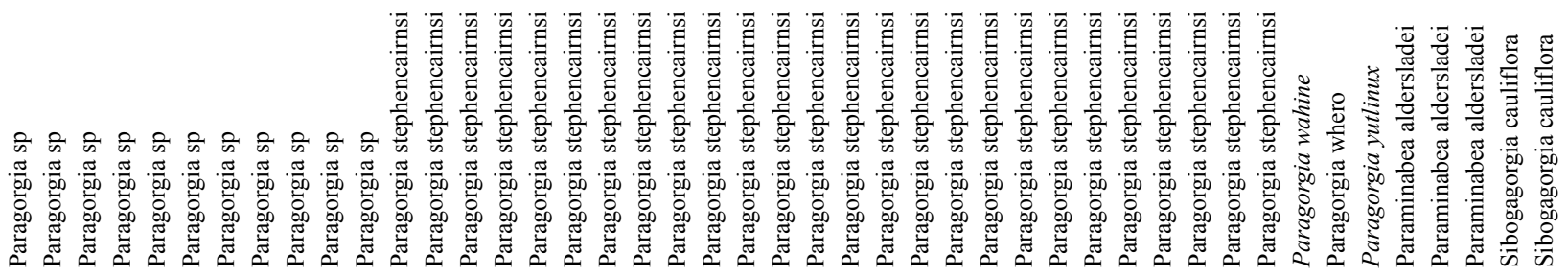




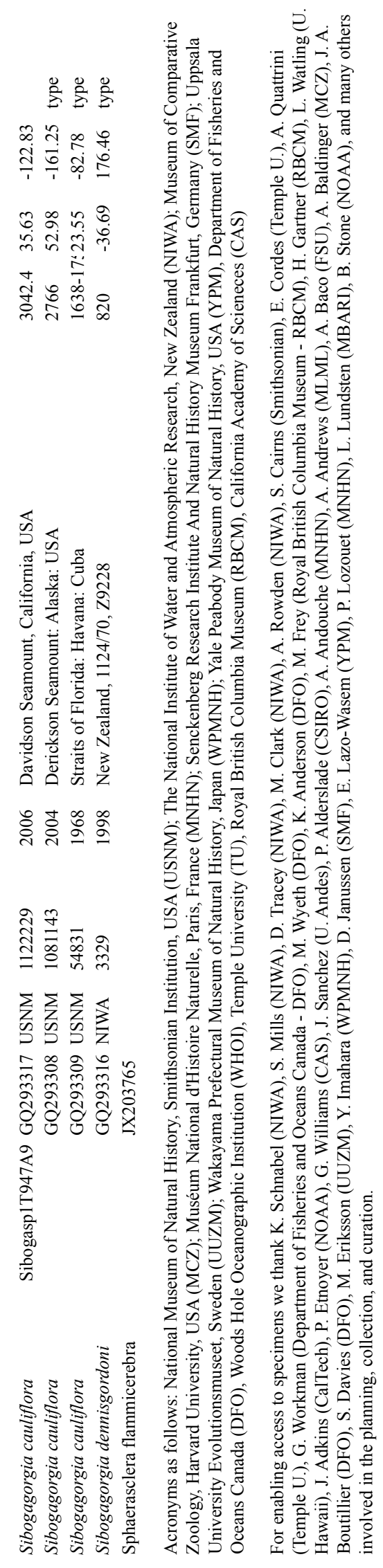




\title{
CHAPTER 6
}

\section{The genomics of adaptation potential of deep-sea corals to environmental changes}

\begin{abstract}
Species that live in a wide range of environmental conditions constitute natural experiments of biological adaptations, which can help us to understand possible ecological consequences of environmental changes on ecosystems. A few populations of some deep-sea coral species can be found in shallow $(<45 \mathrm{~m})$ highlatitude fjord environments, where they experience significantly different environmental conditions than their deep relatives. Therefore, these shallow-water populations are believed to inhabit the extremes of the species' physiological tolerances and likely have developed adaptations that enable them to colonize these shallow-water environments. Here, we aim to identify genomic regions that may have enabled the successful adaptation to shallow-water in the deep-sea octocoral species Paragorgia stephencairnsi. To characterize the genome-wide genetic diversity of populations of P. stephencairnsi found in shallowwater populations and compare it to the genetic diversity from deep-water populations, we performed high-resolution genome-wide scans of single nucleotide polymorphisms. We find patterns of significant population genetic differentiation among the examined populations of $P$. stephencairnsi, which are consistent with the hypothesis that larvae from outer deep populations seeded shallow-water inner fjord populations. Furthermore, we find candidate positive-selection markers shared between parallel comparisons of two shallow populations and a deep populations, and thus identify them as likely candidate makers for genomic regions involved in adaptation to the shallow-water fjord environment. This study lays groundwork for describing the impacts of natural selection on deep-sea coral species in the face of environmental changes.
\end{abstract}

\section{INTRODUCTION}

Species that live in a wide range of environmental conditions constitute natural experiments of biological adaptations, which can help us to understand possible ecological consequences of environmental changes on ecosystems (e.g. Emerson et al. 2010). Populations of deep-sea coral species typically live hundreds or 
thousands of meters below the surface in the relatively uniform environmental conditions characteristic of the deep-sea (Roberts et al. 2009). However, a few populations of some deep-sea coral species can be found in shallow $(<45 \mathrm{~m})$ high-latitude fjord environments, where low light penetration and cool temperatures presumably create suitable living conditions similar to those found in the deep-sea. Despite the seeming similarities between deep-sea and shallow fjord environments there are significant differences that make these shallow fjords a novel environment for deep-sea species, namely lower hydrostatic pressure and significantly greater ranges of variability for temperature, $\mathrm{pH}$, salinity, current speeds, and sedimentation rates. Therefore, these shallow populations of predominantly deep-sea coral species are believed to inhabit the extremes of the species' physiological tolerances.

Differences in environmental conditions over space and time can have strong selective effects on natural populations by modifying the survival and reproductive success of individuals, and thus altering genetic composition of the populations and their ability to respond to environmental changes (e.g., Prada \& Hellberg 2013). When isolated populations of a species are exposed to similar selective pressures, e.g. deep-sea coral populations in semi-enclosed shallow environments such as inlets and fjords, they tend to develop similar solutions to common challenges - a process known as parallel adaptation (e.g., Chan et al. 2010; Hohenlohe et al. 2010; Jones et al. 2012; Miller et al. 2012). Hohenlohe et al. (2010) present an exemplary case exploring the genome-wide consequences of this evolutionary process. In that study the authors investigate the parallel adaptation to freshwater environments in marine stickleback populations by performing high-resolution genomic scans of single nucleotide polymorphisms (SNPs) from ancestral marine and derived freshwater populations. When genome-mapped data from marine populations are compared to freshwater populations it is possible to detect specific regions in the genome that had more differentiation than what is expected under neutrality. Common regions of differentiation across multiple populations are identified as strong candidates for parallel adaptation of populations of a marine species to freshwater. Hohenlohe et al. (2010) show that several genomic regions identified through this method co-localize with previously identified quantitative trait loci (regions of the genome that account for particular observable characteristics of organisms), thus demonstrating the usefulness of this approach for the identification of ecologically important genes.

Bubblegum corals (Paragorgiidae, Octocorallia) are among the most abundant and widely distributed benthic foundation species in deep-water ecosystems worldwide (Roberts et al. 2009; Wating et al. 2011). They play an important ecological role, akin to the structural role of large trees in a rainforest, by generating three-dimensional habitats for a great number of micro- and macro-organisms (BuhlMortensen \& Mortensen 2004; Auster et al. 2005; Buhl-Mortensen \& Mortensen 2005; DeVogelaere et 
al. 2005; Nedashkovskaya et al. 2005). Populations of the bubblegum coral species Paragorgia stephencairnsi Sánchez, 2005 (sensu Herrera and Shank (Chapter 5)) are typically found in the ocean at depths greater than 200 meters, attached to hard grounds on the continental shelf and slope, and seamounts along the western coast of North America (Sánchez 2005). A few populations of this species inhabit shallow fjords (as shallow as 30 meters) at the northern boundary of its distribution. Recent phylogenomic evidence shows $P$. stephencairnsi evolved from deep-sea ancestors (Herrera \& Shank Chapter 5). Thus, the most parsimonious scenario is that shallow-water populations of $P$. stephencairnsi in high-latitude fjords originated from colonization seeded by deeper populations.

a)
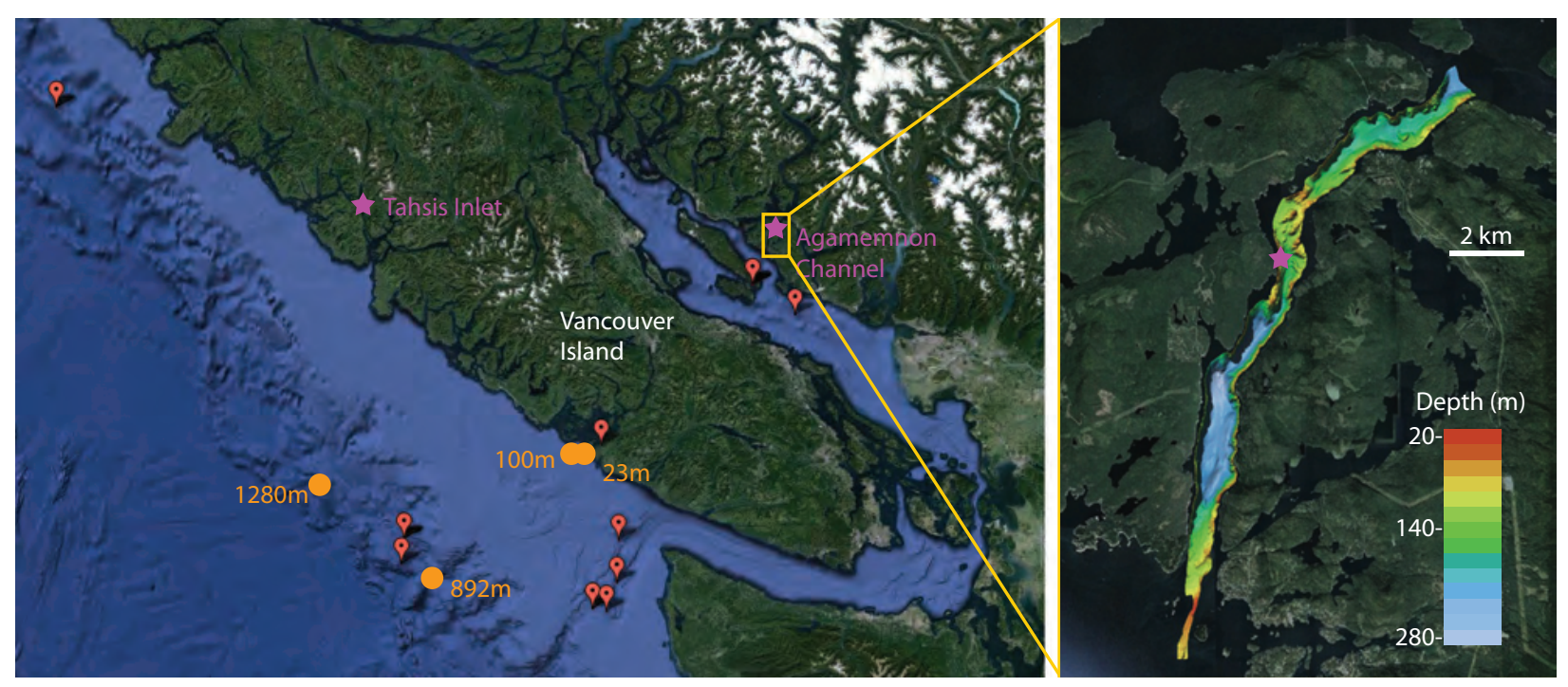

b)

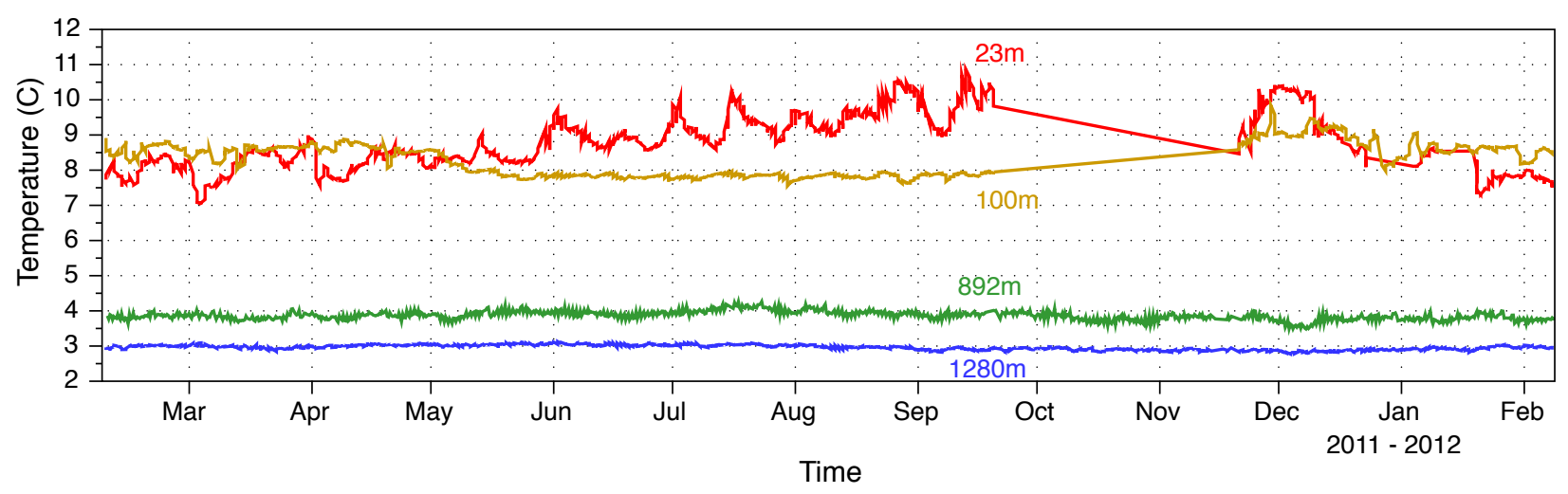

Figure 1. a) Geographic location of P. stephencairnsi populations off British Columbia, Canada. Left map shows sites where samples have been obtained. Purple stars indicate locations of shallow populations (accesible via scuba diving). Red flags indicate the locations of deep populations. Orange dots indicate observatory nodes from the NorthEast Pacific Time-Series Undersea Networked Experiments (NEPTUNE) monitoring environmental variables 
at $23 \mathrm{~m}, 100 \mathrm{~m}, 892 \mathrm{~m}$, and $1280 \mathrm{~m}$ depths. Right map shows the bathymetry of the glacially formed Agamemnon Channel, one of the primary shallow-water fjord collection sites. b) Temperature records from monitoring nodes at four different depths off the North Western coast of North America. Location of nodes shown in Fig. 3a. Data courtesy of NEPTUNE ocean network observatory (Canada).

In general, fjords -long and narrow coastal sea inlets formed by glaciers - can be thought of as semienclosed marine basins as they are limited at their sides by landmasses. The seafloor in fjords dramatically shoals at their mouths because of the paleoglaciers' terminal moraines (see Agamemnon Channel example in Fig. 1a). The geological characteristics of fjords constrain the circulation and exchange between these semi-enclosed environments and the 'outer' ocean environment. Thus, the amounts of gene flow between 'outer' deep and 'inner' shallow populations of marine organisms, such as Paragorgia stephencairnsi, are likely limited by the these same geomorphic features.

The main goal of this study is to identify and characterize potential genomic regions that have enabled the successful colonization of shallow-water environments from the deep-sea by $P$. stephencairnsi. We hypothesize that semi-isolated shallow water populations of $P$. stephencairnsi in different fjord systems have independently evolved adaptations in parallel to cope with distinct highly variable conditions of the surface ocean. As a result the expectation is that, in each population, the environment has independently selected common sets of genetic diversity. This would leave characteristic signatures of parallel differentiation in their genomes. Here, we perform high-resolution genome-wide scans of SNPs to characterize the genome-wide genetic diversity of populations of P. stephencairnsi found in shallowwater populations, and compare it to the genetic diversity from deep-water populations. Through these comparisons we identify patterns of differentiation that would be indicative of non-random evolutionary processes of natural selection and adaptation and lay groundwork for describing the impacts of natural selection on deep-sea coral species in the face of environmental changes.

\section{METHODS}

Specimens of P. stephencairnsi from shallow water populations (less than 45m; Agamemnon Channel and Tahsis Strait) were collected in May 4-17, 2013 in British Columbia, Canada, during 8 decompression SCUBA dives. Specimens from deep populations around Vancouver Island were collected in various oceanographic expeditions by collaborators at Memorial University, the Department of Fisheries and Oceans Canada, the Royal British Columbia Museum, and the National Oceanographic and Atmospheric Administration (Table 1). 
Table 1. Collection information for the specimens used in this study.

\begin{tabular}{|c|c|c|c|c|c|c|c|c|}
\hline ID & Collection & Catalog Number & Date & Locality & $\begin{array}{l}\text { Depth } \\
\text { (m) }\end{array}$ & Lat. & Lon. & Population \\
\hline Agam & WHOI & Agam & 2012 & Agamemnon Channel & 32 & 49.72 & -124.05 & Agamemnon \\
\hline $\mathrm{C} 02$ & WHOI & $\mathrm{C} 02$ & 2013 & Agamemnon Channel; Dive02 & 41 & 49.74 & -124.03 & Agamemnon \\
\hline $\mathrm{C} 03$ & WHOI & $\mathrm{C} 03$ & 2013 & Agamemnon Channel; Dive02 & 41 & 49.74 & -124.03 & Agamemnon \\
\hline $\mathrm{C} 04$ & WHOI & $\mathrm{C} 04$ & 2013 & Agamemnon Channel; Dive02 & 41 & 49.74 & -124.03 & Agamemnon \\
\hline $\mathrm{C} 05$ & WHOI & $\mathrm{C} 05$ & 2013 & Agamemnon Channel; Dive02 & 41 & 49.74 & -124.03 & Agamemnon \\
\hline $\mathrm{C} 07$ & WHOI & $\mathrm{C} 07$ & 2013 & Agamemnon Channel; Dive02 & 41 & 49.74 & -124.03 & Agamemnon \\
\hline $\mathrm{C} 08$ & WHOI & $\mathrm{C} 08$ & 2013 & Agamemnon Channel; Dive02 & 41 & 49.74 & -124.03 & Agamemnon \\
\hline $\mathrm{C} 11$ & WHOI & $\mathrm{C} 11$ & 2013 & Agamemnon Channel; Dive02 & 41 & 49.74 & -124.03 & Agamemnon \\
\hline $\mathrm{C} 12$ & WHOI & $\mathrm{C} 12$ & 2013 & Agamemnon Channel; Dive02 & 41 & 49.74 & -124.03 & Agamemnon \\
\hline $\mathrm{C} 100$ & WHOI & $\mathrm{C} 100$ & 2013 & Vancouver Island, Tahsis Inlet; Dive07 & 40 & 49.86 & -126.67 & Tahsis \\
\hline $\mathrm{C} 101$ & WHOI & $\mathrm{C} 101$ & 2013 & Vancouver Island, Tahsis Inlet; Dive07 & 40 & 49.86 & -126.67 & Tahsis \\
\hline $\mathrm{C} 102$ & WHOI & $\mathrm{C} 102$ & 2013 & Vancouver Island, Tahsis Inlet; Dive07 & 40 & 49.86 & -126.67 & Tahsis \\
\hline $\mathrm{C} 103$ & WHOI & $\mathrm{C} 103$ & 2013 & Vancouver Island, Tahsis Inlet; Dive07 & 40 & 49.86 & -126.67 & Tahsis \\
\hline $\mathrm{C} 104$ & WHOI & $\mathrm{C} 104$ & 2013 & Vancouver Island, Tahsis Inlet; Dive07 & 40 & 49.86 & -126.67 & Tahsis \\
\hline $\mathrm{C} 105$ & WHOI & $\mathrm{C} 105$ & 2013 & Vancouver Island, Tahsis Inlet; Dive07 & 40 & 49.86 & -126.67 & Tahsis \\
\hline $\mathrm{C} 106$ & WHOI & $\mathrm{C} 106$ & 2013 & Vancouver Island, Tahsis Inlet; Dive07 & 40 & 49.86 & -126.67 & Tahsis \\
\hline $\mathrm{C} 107$ & WHOI & $\mathrm{C} 107$ & 2013 & Vancouver Island, Tahsis Inlet; Dive07 & 40 & 49.86 & -126.67 & Tahsis \\
\hline FOC25 & $\mathrm{DFO}$ & 25 & 2012 & W of Graham Island; 2012-65 & 204 & 53.31 & -133.03 & Deep \\
\hline FOC26 & DFO & 26 & 2012 & W of Graham Island; 2012-65 & 221 & 53.30 & -133.04 & Deep \\
\hline FOC30 & DFO & 30 & 2012 & W of Graham Island; 2012-65 & 318 & 53.48 & -133.07 & Deep \\
\hline FOC5 & DFO & 5 & 2009 & E of Graham Island; 2009-47 & 201 & 52.13 & -128.90 & Deep \\
\hline L139 & MU & R1513-L1-0039 & & $\mathrm{S}$ of Texada Island & 268 & 49.50 & -124.17 & Deep \\
\hline L219 & MU & R1513-L2-0019 & & $\mathrm{S}$ of Texada Island & 267 & 49.50 & -124.17 & Deep \\
\hline L341 & MU & R1513-L3-0041 & & $\mathrm{S}$ of Texada Island & 268 & 49.50 & -124.17 & Deep \\
\hline PR27 & MU & R1513-PR-0027 & & $\mathrm{S}$ of Texada Island & 270 & 49.50 & -124.17 & Deep \\
\hline 1122479 & USNM & 1122479 & 2008 & LaPush, west of, Washington & 269 & 48.13 & -125.10 & Deep \\
\hline 1124300 & USNM & 1124300 & 2006 & Vancouver Island, Ohiat Island & 188 & 48.83 & -125.13 & Deep \\
\hline
\end{tabular}

Acronyms as follows: National Museum of Natural History, Smithsonian Institution, USA (USNM); Department of Fisheries and Oceans Canada (DFO), Woods Hole Oceanographic Institution (WHOI), Memorial University (MU).

\section{Molecular laboratory methods}

To characterize the genome-wide genetic diversity of populations of $P$. stephencairnsi, we performed high-resolution genomic scans and identified single nucleotide polymorphisms (SNPs) from restriction site-associated DNA markers (RAD tags) (Baird et al. 2008; Hohenlohe et al. 2010). In short, the RAD sequencing method consists of: 1) the digestion of genomic DNA for each individual with a restriction enzyme; 2) ligation of the resulting fragments to sequencing adapters with unique barcodes for each individual; 3) size-selection and enrichment of the fragments successfully ligated to the adapters; and 4) sequencing via a high-throughput platform (Illumina HiSeq 2000). We performed RAD sequencing with the 6-cutter restriction enzyme PstI, which is predicted to cut between 32,000 and 110,000 times in the 
genome of an octocoral (Herrera \& Shank Chapter 5). This predicted range was obtained using the observed frequency of the PstI recognition sequence, and its probability using a trinucleotide composition model, in the genomes of the cnidarians Nematostella vectensis, Acropora digitifera, Hydra vulgaris, and Alatina moseri (Herrera et al. Chapter 2). Genome size range of 0.3-0.5 pg was used based on observations obtained through flow cytometry in gorgoniid octocorals by Luisa Dueñas at the Universidad de los Andes, Bogotá, Colombia (personal communication).

Total genomic DNA was purified from specimens as in Herrera and Shank (Chapter 5) by: (1) digesting the tissue in 2\% CTAB buffer (Teknova) with proteinase K and RNAse A/T1 (Fermentas) for 1 hour, (2) separating nucleic acids with chloroform: isoamyl alcohol (24:1) (Fermentas) and phenol: chloroform: isoamyl alcohol (25:24:1, Tris buffered at pH 8.0) (Fermentas), (3) precipitating nucleic acids with 100\% ethanol (1:1 volume ratio), and (4) washing the precipitate twice with $70 \%$ ethanol. Concentrationnormalized genomic DNA was submitted to Floragenex Inc. (Eugene, OR) for library preparation and RAD sequencing. Libraries were sequenced by 48-multiplex, using 10-base pairs long barcodes, on a single lane of an Illumina Hi-Seq 2000 sequencer.

\section{Data filtering}

Sequence reads were de-multiplexed and quality filtered with the process_radtags program from the package Stacks v1.19 (Catchen et al. 2013b). Barcodes and Illumina adapters were excluded from each read and length was truncated to 91bp (-t 91) Reads with ambiguous bases were discarded (-c). Reads with an average quality score below 10 (-s 10) within a sliding window of $15 \%$ of the read length (-w 0.15 ) were discarded (-r). The rescue barcodes and RAD-tags algorithm was enabled (-r).

\section{De novo loci assembly}

We performed de novo assemblies of RAD loci using the denovo_map pipeline in Stacks. A minimum depth of three reads per stack was enforced (-m 3). Significantly high-repetitive stacks were discarded by implementing the deleveraging algorithm (-t), as these likely represent sequencing errors, duplications or repetitive regions. No mismatches among loci were allowed when creating the catalog of all the loci identified among the sampled individuals (-n 0). A maximum number of two mismatches was allowed among loci within each individual (-M 2). The maximum number of stacks at a single locus was set to three (--max_locus_stacks 3). 


\section{Demographic Inferences}

We estimated population genetic descriptive statistics per SNP (nucleotide diversity $\pi$, proportion of polymorphic loci, observed heterozygosity, minor allele frequency, number of private alleles, inbreeding index $\mathrm{F}_{\mathrm{IS}}$, and population differentiation index $\mathrm{F}_{\mathrm{ST}}$ ) using the program populations of Stacks. We only analyzed loci that were present in all populations of each species (-p) and in all individuals in each population (-r). We calculated population $\mathrm{F}_{\mathrm{ST}}$ values utilizing a $p \_$value filter (-f) to keep only significant estimates $(\alpha=0.05)$. We exported SNP data in genpop format, keeping only one SNP per locus to avoid violating the assumption of independence among markers in downstream analyses.

To summarize the variation in the SNP data among individuals and populations we a performed principal component analyses (PCA) as in (Reitzel et al. 2013), using the program smartpca from the package Eigensoft v5.0 (Patterson et al. 2006; Price et al. 2006). We evaluated the significance of the identified principal components through Tracy-Widom statistics (Tracy \& Widom 1994; Johnstone 2001) and evaluated the statistical significance of the differences between populations with a chi-square test.

\section{Candidate adaptation markers}

We identified markers linked to candidate genomic regions involved in adaptation to shallow water environments from the deep-sea by detecting $F_{S T}$ outliers (i.e., allelic frequencies between deep and shallow populations that show greater differentiation than expected under a neutral model of evolution, characterized by the accumulation of random mutations that do not affect survival and reproduction of organisms) with the program LOSITAN (Beaumont \& Nichols 1996; Antao et al. 2008). Population genomics theory predicts that these outlier variants will be indicative of genomic regions containing genes or regulatory elements that have been subject to natural selection (Lewontin \& Krakauer 1973; Beaumont $\&$ Balding 2004). SNP positions with outlier $F_{\mathrm{ST}}$ values (those above the 97.5 percentile of the neutral distribution of $\mathrm{F}_{\mathrm{ST}}$ ) were considered indicative of loci subject to natural selection. We considered outliers shared among shallow populations as indicative of parallel adaptations to the shallow-water environment, whereas outliers unique to a particular population were considered as likely indicators of local adaptations (see Hohenlohe et al. 2010).

Genomic sequences of markers under potential positive selection were scanned for functionality by querying against annotated databases of gene models from cnidarian genomes (Nematostella vectensis and Hydra magnipapillata) using BLAST searches at the U.S. National Center for Biotechnology Information (NCBI) databases. Gene Ontology (GO) and Kyoto Encyclopedia of Genes and Genomes 
(KEGG) pathway assignments were attempted for each gene-model match using the program Blast2GO (Conesa et al. 2005).

Table 2. RAD sequencing results, filtering and de novo assebly statistics.

\begin{tabular}{|c|c|c|c|c|c|c|c|}
\hline Population & RAD-seq data file ID & $\begin{array}{l}\text { Total } \\
\text { sequenced } \\
\text { reads }\end{array}$ & $\begin{array}{l}\text { Retained } \\
\text { reads }\end{array}$ & $\begin{array}{l}\text { Percentage of } \\
\text { retained reads } \\
\text { after filtering }\end{array}$ & $\begin{array}{l}\text { Number of } \\
\text { Stacks }\end{array}$ & $\begin{array}{l}\text { Mean } \\
\text { coverage } \\
\text { depth }\end{array}$ & $\begin{array}{l}\text { S.D. of } \\
\text { coverage } \\
\text { depth }\end{array}$ \\
\hline Agamemnon & PAR_Agam_BC & $4,322,564$ & $4,062,469$ & 94.0 & 142,874 & 25.3 & 151.6 \\
\hline Agamemnon & PAR_C02_BCS & $2,149,938$ & $1,594,818$ & 74.2 & 110,403 & 10.8 & 35.7 \\
\hline Agamemnon & PAR_C03_BCS & $2,815,327$ & $2,264,951$ & 80.5 & 126,442 & 13.7 & 73.8 \\
\hline Agamemnon & PAR_C04_BCS & $3,240,713$ & $2,754,373$ & 85.0 & 133,631 & 15.9 & 87.3 \\
\hline Agamemnon & PAR_C05_BCS & $3,465,396$ & $2,972,548$ & 85.8 & 136,642 & 16.9 & 104.3 \\
\hline Agamemnon & PAR_C07_BCS & $2,827,394$ & $2,446,736$ & 86.5 & 129,688 & 14.5 & 59.6 \\
\hline Agamemnon & PAR_C08_BCS & $2,552,442$ & $2,104,566$ & 82.5 & 125,331 & 12.5 & 50.9 \\
\hline Agamemnon & PAR_C11_BCS & $2,763,403$ & $2,286,010$ & 82.7 & 128,412 & 13.6 & 58.6 \\
\hline Agamemnon & PAR_C12_BCS & $2,390,479$ & $2,062,541$ & 86.3 & 124,553 & 12.5 & 53.5 \\
\hline Deep & PAR_1122479_BC & $4,607,551$ & $4,331,474$ & 94.0 & 142,445 & 27.2 & 108.6 \\
\hline Deep & PAR_1124300_WA & $3,843,250$ & $3,627,391$ & 94.4 & 136,241 & 23.7 & 152.2 \\
\hline Deep & PAR_FOC25_BCD & $5,479,109$ & $4,693,804$ & 85.7 & 152,378 & 24.2 & 102.9 \\
\hline Deep & PAR_FOC26_BCD & $5,242,949$ & $4,528,629$ & 86.4 & 210,583 & 14.9 & 76.2 \\
\hline Deep & PAR_FOC30_BCD & $4,417,520$ & $3,781,973$ & 85.6 & 174,618 & 15.8 & 116.6 \\
\hline Deep & PAR_FOC5_BCD & $2,834,377$ & $2,455,041$ & 86.6 & 135,156 & 13.3 & 129.2 \\
\hline Deep & PAR_L139_BCD & $2,928,518$ & $2,392,739$ & 81.7 & 129,058 & 14.1 & 62.6 \\
\hline Deep & PAR_L219_BCD & $1,752,674$ & $1,289,295$ & 73.6 & 96,904 & 9.5 & 51.0 \\
\hline Deep & PAR_L341_BCD & $3,253,979$ & $2,761,200$ & 84.9 & 133,647 & 16.1 & 75.8 \\
\hline Deep & PAR_PR27_BCD & $3,536,670$ & $2,942,112$ & 83.2 & 135,368 & 17.0 & 72.3 \\
\hline Tahsis & PAR_C100_BCS & $5,998,914$ & $4,984,766$ & 83.1 & 149,979 & 26.1 & 90.1 \\
\hline Tahsis & PAR_C101_BCS & $5,332,619$ & $4,652,535$ & 87.2 & 150,525 & 24.2 & 142.6 \\
\hline Tahsis & PAR_C102_BCS & $4,347,757$ & $3,710,381$ & 85.3 & 141,586 & 20.5 & 73.7 \\
\hline Tahsis & PAR_C103_BCS & $2,272,200$ & $1,706,852$ & 75.1 & 114,196 & 11.0 & 38.5 \\
\hline Tahsis & PAR_C104_BCS & $2,720,994$ & $2,203,568$ & 81.0 & 124,966 & 13.5 & 56.5 \\
\hline Tahsis & PAR_C105_BCS & $2,778,573$ & $2,003,812$ & 72.1 & 123,158 & 12.1 & 45.9 \\
\hline Tahsis & PAR_C106_BCS & $2,565,150$ & $2,116,517$ & 82.5 & 123,271 & 13.1 & 59.1 \\
\hline \multirow[t]{3}{*}{ Tahsis } & PAR_C107_BCS & $2,697,054$ & $2,189,475$ & 81.2 & 125,420 & 13.3 & 45.6 \\
\hline & AVERAGE & $3,449,538$ & $2,922,984$ & 83.7 & 135,462 & 16.5 & 80.5 \\
\hline & S.D. & $1,136,671$ & $1,066,781$ & 5.6 & 21,081 & 5.2 & 34.5 \\
\hline
\end{tabular}

\section{RESULTS}

\section{RAD-seq produced high-quality sequence data}

We generated restriction site associated DNA sequence (RAD-seq) data for 27 individuals of $P$. stephencairnsi collected in the British Columbia region (Table 2). We obtained approximately $3.5 \pm 1.1$ 
(mean \pm standard deviation) million sequence reads per individual (100bp length), with individual values ranging from 1.75 to 6.0 million reads. Approximately $83.7 \pm 5.6 \%$ of these were retained after quality filters. De novo loci assemblies produced approximately $135 \pm 21$ thousand unique sequence stacks per individual, with a mean coverage depth of $16.5 \pm 5.2 \mathrm{X}$.

Table 3. Population genetic statistics calculated from only variant positions, and from both variant and fixed positions. Values indicate means \pm standard deviation

\begin{tabular}{|c|c|c|c|c|c|c|c|}
\hline Population & $\begin{array}{l}\text { Private } \\
\text { alleles }\end{array}$ & $\begin{array}{l}\text { Variant } \\
\text { sites }\end{array}$ & $\begin{array}{l}\text { \% polym. } \\
\text { sites }\end{array}$ & $\begin{array}{l}\text { Major allele } \\
\text { frequency }\end{array}$ & $\begin{array}{l}\text { Observed } \\
\text { heterozygosity }\end{array}$ & $\begin{array}{l}\text { Nucleotide } \\
\text { diversity }(\pi)\end{array}$ & Fis \\
\hline Agamemnon & 2,713 & 17,074 & 65.23 & $0.8737 \pm 0.1517$ & $0.2149 \pm 0.2604$ & $0.1858 \pm 0.1884$ & $-0.0612 \pm 0.2404$ \\
\hline Tahsis & 2,000 & 17,071 & 55.54 & $0.8793 \pm 0.1578$ & $0.2000 \pm 0.2737$ & $0.1743 \pm 0.1975$ & $-0.0468 \pm 0.2717$ \\
\hline Deep & 3,046 & 17,062 & 69.35 & $0.8697 \pm 0.1523$ & $0.2102 \pm 0.2565$ & $0.1904 \pm 0.1865$ & $-0.0340 \pm 0.2851$ \\
\hline
\end{tabular}

All positions (variant and fixed)

\begin{tabular}{|c|c|c|c|c|c|c|c|}
\hline Population & $\begin{array}{l}\text { Private } \\
\text { alleles }\end{array}$ & Sites & $\begin{array}{l}\text { \% polym. } \\
\text { sites }\end{array}$ & $\begin{array}{l}\text { Major allele } \\
\text { frequency }\end{array}$ & $\begin{array}{l}\text { Observed } \\
\text { heterozygosity }\end{array}$ & $\begin{array}{l}\text { Nucleotide } \\
\text { diversity }(\pi)\end{array}$ & Fis \\
\hline Agamemnon & 2,713 & $1,873,995$ & 0.59 & $0.9988 \pm 0.0200$ & $0.0020 \pm 0.0316$ & $0.0017 \pm 0.0245$ & $-0.0006 \pm 0.0245$ \\
\hline Tahsis & 2,000 & $1,873,999$ & 0.51 & $0.9989 \pm 0.0200$ & $0.0018 \pm 0.0316$ & $0.0016 \pm 0.0245$ & $-0.0004 \pm 0.0265$ \\
\hline Deep & 3,046 & $1,873,984$ & 0.63 & $0.9988 \pm 0.0200$ & $0.0019 \pm 0.0316$ & $0.0017 \pm 0.0245$ & $-0.0003 \pm 0.0283$ \\
\hline
\end{tabular}
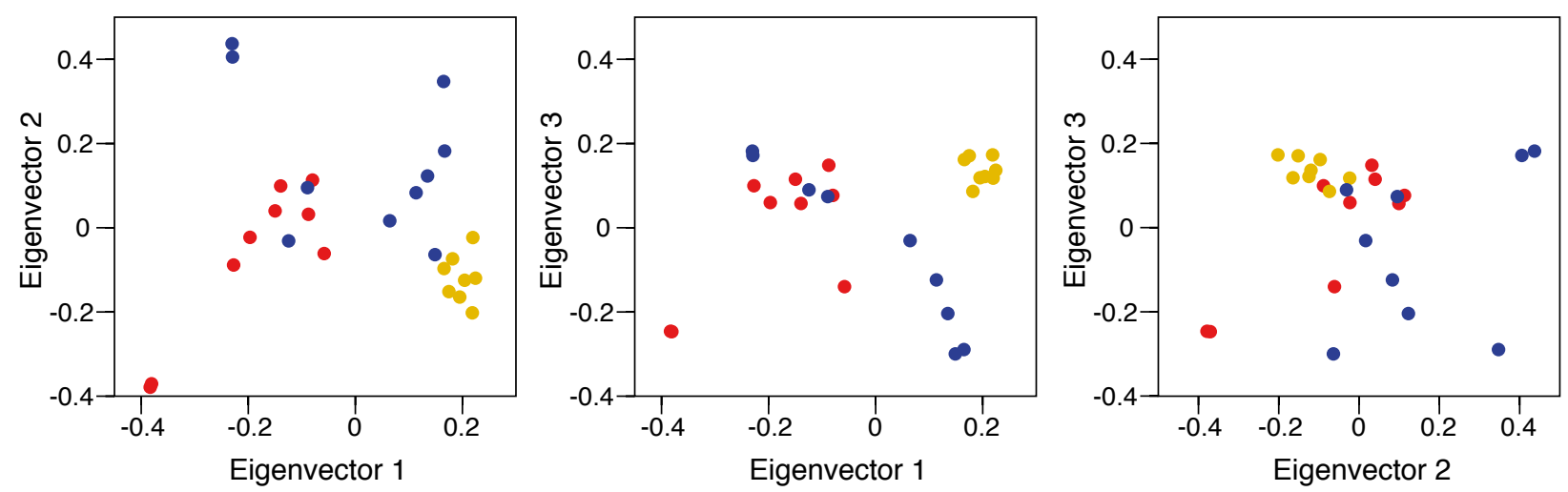

Figure 2. Principal component analysis of genetic variation from SNP in Paragorgia stephencairnsi from British Columbia. Each dot represents an individual. Colors indicate the source population: Agamemnon (red), Tahsis (yellow), and Deep (blue). The three principal axes of variation (eigenvectors) are shown. 


\section{Significant population differentiation among all populations}

There were 10,920 loci shared among all individuals in all populations. These loci contained over 17 thousand SNPs, for an average of 1.56 SNP per locus (Table 3). The three largest axes of variation (eigenvectors) identified from the principal components analysis of SNP data revealed clear separation between the two shallow-water populations Tahsis and Agamemnon, but evident overlap between the individuals from the Deep population and both of the shallow (Fig. 3). All eigenvectors were statistically significant $(P<0.001, P=0.002$, and $P=0.031$ for eigenvectors 1,2 and 3, respectively; $\alpha=0.05)$. All differences among populations were also statistically significant (Agamemnon vs. Tahsis: $\chi^{2}=40.1$, $P<0.001$; Agamemnon vs. Deep: $\chi^{2}=16.4, P=0.003$; Tahsis vs. Deep: $\chi^{2}=30.3, P<0.001 ; \alpha=0.05$ ). Mean pairwise $F_{S T}$ values indicate that population differentiation is significantly greater between the two shallow-water populations Tahsis and Agamemnon $\left(\mathrm{F}_{\mathrm{ST}}=0.0519, P<0.05\right)$, than between either shallowwater population and the Deep population (Agamemnon vs. Deep: $\mathrm{F}_{\mathrm{ST}}=0.0354, P<0.05$; Tahsis vs. Deep: $\left.\mathrm{F}_{\mathrm{ST}}=0.0430, P<0.05\right)$.

\section{Differences in nucleotide diversity between shallow and deep-populations}

Summary population genetic statistics (private alleles, percentage of polymorphic sites, and nucleotide diversity) calculated from variant positions revealed higher diversity in the Deep population than in either of the shallow-water populations. These same metrics indicated that the Tahsis shallow-water population has the lowest genetic diversity of all three examined populations. Differences in population genetic diversity were not evident when summary population genetic statistics were calculated from both fixed and variant positions.

\section{Differences in allele frequency distributions between shallow and deep populations}

Minor allele frequency spectra show that a majority of the alleles in each population have low frequencies

(Fig. 2), as expected for population near mutation-drift equilibrium. Shallow-water populations show a small modal shift towards higher allelic frequencies (particularly in Tahsis) and a noticeable increase of intermediate frequency alleles, compared to the deep population. $\mathrm{F}_{\text {IS }}$ distributions for all populations were centered on zero, with a tendency to negative values, indicating random mating in populations and a slight excess of heterozygotes.

\section{Candidate adaptation markers}

Outlier analyses between shallow-water populations and the Deep population found 733 SNPs candidate positive-selection markers when comparing Agamemnon vs. Deep, and 261 when comparing Tahsis vs. 
Deep. Of these, 63 SNPs candidate positive selection markers were shared in both comparisons, and thus are considered candidate makers for adaptive genomic regions.

a)
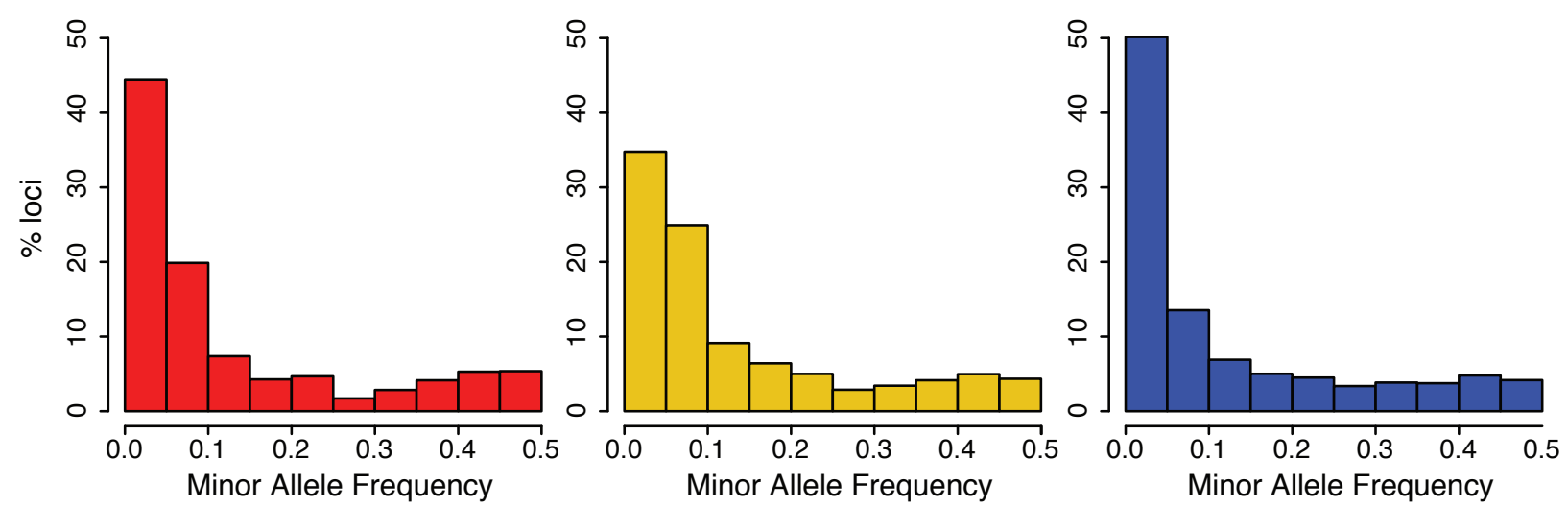

b)
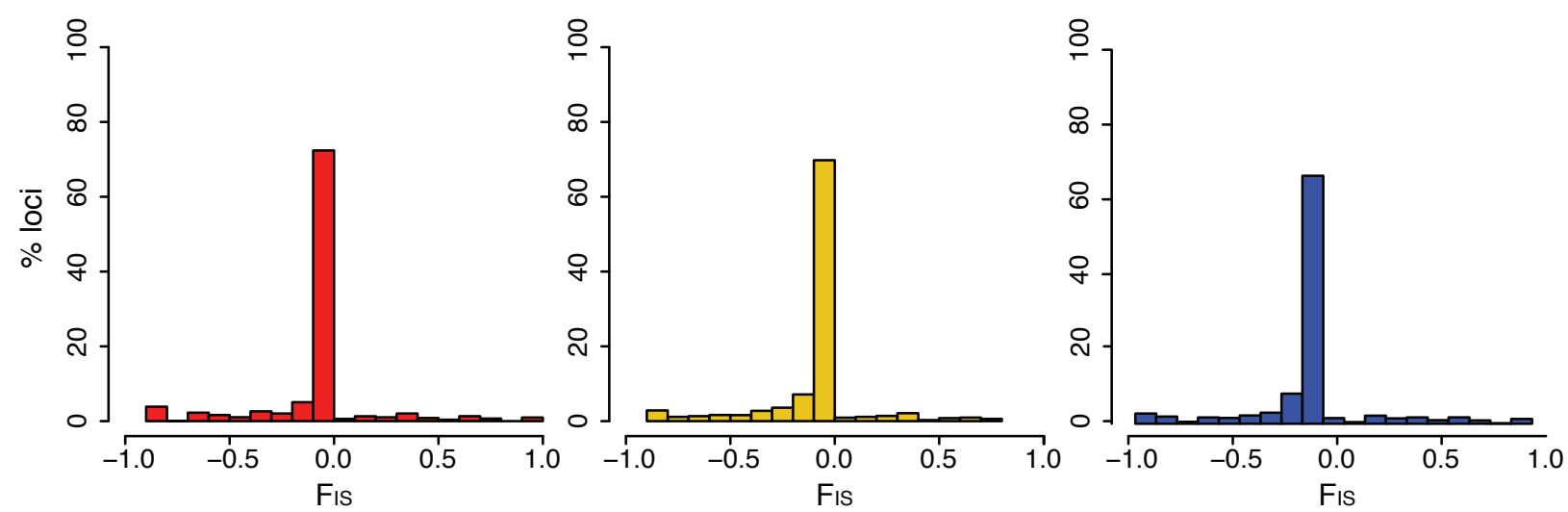

Figure 3. Frequency distributions of population genetic summary statistics for populations of Paragorgia stephencairnsi from British Columbia. a) Minor allele frequency spectra for SNPs loci. b) Frequency spectra of Wright's inbreeding index $F_{\text {IS }}$ values calculated for each SNP locus. Colors indicate the source population: Agamemnon (red), Tahsis (yellow), and Deep (blue).

From the markers identified as potentially adaptive, 16 produced Blast matches to the non-redundant nucleotide NCBI database (Table 4). None produced matches to available protein or gene ontology databases. Many of the matches corresponded to coding DNA sequences of unknown function and uncharacterized mRNAs. The top three marker matches, in terms of Blast E-values (the number of expected false-positive matches in a database of given size), correspond to mRNAs of Hydra magnipapillata: a mitogen-activated protein kinase 7-like mRNA (RAD locus catalog 17484) potentially involved in cellular cycles and cell differentiation; a phosphoribosylformylglycinamidine synthase partial mRNA (RAD locus catalog 23843) potentially involved in purine metabolism; and a zinc finger protein 
41-like mRNA (RAD locus catalog 83962) potentially a transcription factor associated with meiosis in spermatogenesis.

Table 4. Blast results for the candidate positive selection markers shared between comparisons of shallow-water populations vs. Deep population. Only results that produced hits in the non-redundant nucleotide NCBI database are shown.

\begin{tabular}{|c|c|c|c|c|c|}
\hline $\begin{array}{l}\text { RAD } \\
\text { locus } \\
\text { catalog } \\
\text { name }\end{array}$ & Blast hit species & Blast hit sequence description & $\begin{array}{l}\text { Blast } E \text { - } \\
\text { value }\end{array}$ & $\begin{array}{l}\text { Blast hit } \\
\text { accession }\end{array}$ & $\begin{array}{l}\text { Blast hit } \\
\% \\
\text { similarity }\end{array}$ \\
\hline 17484 & Hydra magnipapillata & mitogen-activated protein kinase 7 -like partial mrna & $1.00 \mathrm{E}-08$ & XM004210406 & 75 \\
\hline 23843 & Hydra magnipapillata & phosphoribosylformylglycinamidine synthase partial mrna & $3.60 \mathrm{E}-08$ & XM001641610 & 77 \\
\hline 83962 & Hydra magnipapillata & zinc finger protein 41 -like mrna & $2.80 \mathrm{E}-03$ & XM002162877 & 80 \\
\hline 57130 & Hydra magnipapillata & uncharacterized loc100207636 partial mrna & $9.60 \mathrm{E}-03$ & XM002158158 & 79 \\
\hline 26651 & Nematostella vectensis & protein partial mrna & $9.60 \mathrm{E}-03$ & XM001627193 & 83 \\
\hline 843 & Nematostella vectensis & gene for complete cds & $1.20 \mathrm{E}-01$ & BR000671 & 79 \\
\hline 36131 & Nematostella vectensis & protein partial mrna & 4.10E-01 & XM001629268 & 81 \\
\hline 86101 & Hydra magnipapillata & uncharacterized loc101234456 mrna & $1.40 \mathrm{E}+00$ & XM004211848 & 78 \\
\hline 101291 & Hydra magnipapillata & uncharacterized loc $101240885 \mathrm{mrna}$ & $1.40 \mathrm{E}+00$ & XM004206580 & 80 \\
\hline 22838 & Hydra magnipapillata & lysine-specific demethylase $6 a-$ like mrna & $1.40 \mathrm{E}+00$ & XM002167270 & 83 \\
\hline 67285 & Hydra magnipapillata & uncharacterized loc100199733 mrna & $1.40 \mathrm{E}+00$ & XM002159843 & 80 \\
\hline 76378 & Hydra magnipapillata & uncharacterized loc100202739 mrna & $5.00 \mathrm{E}+00$ & XM002162521 & 77 \\
\hline 103847 & Hydra magnipapillata & uncharacterized loc100207904 mrna & $5.00 \mathrm{E}+00$ & XM002160483 & 75 \\
\hline 71485 & Hydra magnipapillata & uncharacterized loc100209560 mrna & $5.00 \mathrm{E}+00$ & XM002160476 & 78 \\
\hline 8691 & Nematostella vectensis & protein partial mrna & $5.00 \mathrm{E}+00$ & XM001627130 & 80 \\
\hline 27970 & Nematostella vectensis & protein complete cds & $5.00 \mathrm{E}+00$ & XM001639122 & 79 \\
\hline
\end{tabular}

\section{DISCUSSION}

\section{Fjord environments effectively isolate marine populations}

In this study, we find significant population genetic differentiation among all examined populations of Paragorgia stephencairnsi. This genetic differentiation is particularly marked between the shallow-water fjord populations as indicated by the lack of overlap among groups in the principal components analysis (PCA) and the elevated population differentiation $\mathrm{F}_{\mathrm{ST}}$ value. Our results indicate that fjords act as semienclosed basins effectively isolating coral populations living within them. The evidence we present conforms to previous results from population genetic studies in pelagic and benthic organisms such as calanoid copepods (Bucklin et al. 2000), glacier lanternfish (Suneetha \& Salvanes 2001), sea stars (Perrin et al. 2004), Pacific cod (Cunningham et al. 2009), sprat (Glover et al. 2011), and Pacific herring (Wildes 
et al. 2011), which show significant population genetic structuring in small spatial scales among fjord populations, and with respect to open ocean populations.

\section{Shallow-water fjord populations originated from the deep}

Taken together, our results are consistent with the hypothesis that larvae from outer deep populations seeded shallow-water inner fjord populations. Smaller genetic diversity found in the shallow-water fjord populations relative to those from the deep population, indicated by smaller spread in the PCA and smaller nucleotide diversity values $(\pi)$, is suggestive of larger effective population size $\left(N_{e}\right)$ in the deep population (given the relationship $\pi=4 N_{e} \mu$, assuming equal mutation rates $\mu$ and mutation-drift equilibrium). The evident, although small, overlap in the PCA between the large deep population and each one of the smaller shallow-water fjord populations suggests limited gene flow from the deep. This colonization from the deep must have occurred relatively recently, given that present-day North American fjords were predominantly occupied by massive glaciers or exposed to the atmosphere due to sea-level change during the last glacial maximum 33,000-14,500 years ago (Clague \& James 2002; Clark \& Mix 2002; Clark et al. 2009). However, we suggest enough time may have passed since colonization because allele frequency distributions are not strongly skewed towards fixation, as expected for young nonequilibrium populations founded by few individuals (e.g., Catchen et al. 2013a). The dynamics of population structuring between shallow-water inner fjord and outer deep populations of $P$. stephencairnsi over time are unknown. However, compelling evidence from other species suggests that these patterns of differentiation between fjord and open water populations can be stable over at least hundreds of years (Harnstrom et al. 2011).

\section{Natural selection in shallow-water fjord environments}

The differences in conditions between open water and shallow fjord environments (e.g. Fig. 1b) can also act as barriers for gene flow, further limiting the amount of gene flow between shallow and deep-sea populations. Compared to deep populations, the shallow-water fjord populations are exposed to lower hydrostatic pressure and significantly greater variability ranges of temperature, $\mathrm{pH}$, salinity, current speeds, and sedimentation rates influenced by marked seasonality (observations from the NEPTUNE and VENUS time series). These conditions may not only act as barriers for gene flow but also as selective forces. The observed $\mathrm{F}_{\mathrm{IS}}$ distributions with a tendency to negative values and the higher percentage of loci of intermediate allelic frequencies in shallow-water populations, relative to the deep population, may be the result of natural selection (e.g. heterozygote advantage or balancing selection). Alternatively, demographic processes, such as bottlenecks, may also produce similar patterns (Luikart et al. 1998). 
The comparative approach between shallow-water populations and the deep ancestral population of $P$. stephencairnsi, allowed us to identify, for the first time, potential markers of parallel adaptation to the shallow-water environment in a deep-sea organism. Although only three markers were mapped to known cnidarian functional regions, there is the potential to identify more genomic regions and link them to potential functions and mechanisms as more genomic resources become available (Hohenlohe et al. 2010; Reitzel et al. 2013). Significant environmental changes are occurring due to anthropogenic $\mathrm{CO}_{2}$ emissions (Hoegh-Guldberg \& Bruno 2010). Despite efforts to understand the effect of these changes on marine species, little is known about the adaptive mechanisms that would allow them to survive over ecological and evolutionary time scales. Shallow-water populations of deep-sea coral species have already adapted to deal with the environmental extremes of the surface ocean, thus they could constitute pre-adapted populations that could expand their range to deeper water in the case of significant environmental changes at depth, thereby seeding future deep-sea ecosystems. On the other hand, shallow-water populations of deep-sea corals may live at the tolerance boundary of their species, and thus could face habitat shifts and local extinction in the near future. A better understanding of the adaptive potential of these corals will allow us to assess the possible impacts of climate change on the diverse but vulnerable ecosystems supported by these habitat-forming corals.

\section{Future work}

To increase the confidence in our demographic and natural selection inferences we plan to perform RAD sequencing on additional available individuals from deep and shallow populations. Additionally, the potential markers of parallel adaptation to the shallow-water environment will be mapped to the draft genome sequence of an P. stephencairnsi individual, which will be generated using high-throughput sequencing and routine algorithms developed to assemble full genomes from short sequence-reads (following Gnerre et al. 2011). High-resolution genomic scans generated by RAD-seq provide genotypic data for tens of thousands of SNPs, thus allowing the creation of genome-wide distributions of $\mathrm{F}_{\mathrm{ST}}$ and other population genetic summary statistics, which allow further identification of candidate potential genomic regions and elements involved in parallel adaptation to the shallow-water environment (see Hohenlohe et al. 2010).

\section{ACKNOWLEDGEMENTS}

This research was supported by the National Geographic Society/Waitt Foundation (W285-13 to SH); the National Oceanic and Atmospheric Administration (NA09OAR4320129 to TS); the National Science Foundation (OCE-1131620 to TS); the National Aeronautics and Space Administration (NNX09AB76G 
to TS); and the Academic Programs Office (Ocean Ventures Fund to SH), the Ocean Exploration Institute (Fellowship support to TMS) and the Ocean Life Institute of the Woods Hole Oceanographic Institution (WHOI). For enabling access to key specimens we thank S. Cairns (Smithsonian), G. Workman (Department of Fisheries and Oceans Canada - DFO), M. Wyeth (DFO), K. Anderson (DFO), M. Frey (Royal British Columbia Museum - RBCM), H. Gartner (RBCM), Ed Bowlby (NOAA, Olympic Coast National Marine Sanctuary), E. Edinger (Memorial U.) and J. Sanchez (U. Andes). We also thank E. O’Brien (WHOI), D. Forsman (WHOI), J. Fellows (WHOI), J. \& S. Schooner, K. Heylar, and N. McDaniel for invaluable assistance during scuba diving fieldwork in British Columbia (DFO scientific license FIN130270). We also thank A. Tarrant, A. Reitzel, and E. Bors for providing helpful comments that improved this manuscript.

\section{REFERENCES}

Antao T, Lopes A, Lopes R, Beja-Pereira A, Luikart G (2008) LOSITAN: A workbench to detect molecular adaptation based on a Fst-outlier method. BMC Bioinformatics 9, 323.

Auster PJ, Moore J, Heinonen KB, Watling L (2005) A habitat classifi cation scheme for seamount landscapes: assessing the functional role of deep-water corals as fish habitat. In: Cold-water Corals and Ecosystems (eds. Freiwald AR, Roberts JM), pp. 761-769. Springer-Verlag, Berlin Heidelberg.

Baird NA, Etter PD, Atwood TS, et al. (2008) Rapid SNP discovery and genetic mapping using sequenced RAD markers. PLoS One 3, e3376.

Beaumont MA, Balding DJ (2004) Identifying adaptive genetic divergence among populations from genome scans. Molecular Ecology 13, 969-980.

Beaumont MA, Nichols RA (1996) Evaluating loci for use in the genetic analysis of population structure. Proceedings of the Royal Society of London Series B-Biological Sciences 263, 1619-1626.

Bucklin A, Kaartvedt S, Guarnieri M, Goswami U (2000) Population genetics of drifting (Calanus spp.) and resident (Acartia clausi) plankton in Norwegian fjords. Journal of Plankton Research 22, 1237-1251.

Buhl-Mortensen L, Mortensen PB (2004) Crustaceans associated with the deep-water gorgonian corals Paragorgia arborea (L., 1758) and Primnoa resedaeformis (Gunn., 1763). Journal of Natural History 38, 1233-1247.

Buhl-Mortensen L, Mortensen PB (2005) Distribution and diversity of species associated with deep-sea gorgonian corals off Atlantic Canada. In: Cold-Water Corals and Ecosystems (eds. Freiwald AR, Roberts JM), pp. 849-879. Springer-Verlag, Berlin Heidelberg.

Catchen J, Bassham S, Wilson T, et al. (2013a) The population structure and recent colonization history of Oregon threespine stickleback determined using restriction-site associated DNA-sequencing. Molecular Ecology 22, 2864-2883.

Catchen J, Hohenlohe PA, Bassham S, Amores A, Cresko WA (2013b) Stacks: an analysis tool set for population genomics. Molecular Ecology 22, 3124-3140.

Chan YF, Marks ME, Jones FC, et al. (2010) Adaptive evolution of pelvic reduction in sticklebacks by recurrent deletion of a Pitx1 enhancer. Science 327, 302-305.

Clague JJ, James TS (2002) History and isostatic effects of the last ice sheet in southern British Columbia. Quaternary Science Reviews 21, 71-87.

Clark PU, Dyke AS, Shakun JD, et al. (2009) The Last Glacial Maximum. Science 325, 710-714. 
Clark PU, Mix AC (2002) Ice sheets and sea level of the Last Glacial Maximum. Quaternary Science Reviews 21, 1-7.

Conesa A, Gotz S, Garcia-Gomez JM, et al. (2005) Blast2GO: a universal tool for annotation, visualization and analysis in functional genomics research. Bioinformatics 21, 3674-3676.

Cunningham KM, Canino MF, Spies IB, Hauser L (2009) Genetic isolation by distance and localized fjord population structure in Pacific cod (Gadus macrocephalus): limited effective dispersal in the northeastern Pacific Ocean. Canadian Journal of Fisheries and Aquatic Sciences 66, 153-166.

DeVogelaere AP, Burton EJ, Trejo T, et al. (2005) Deep-sea corals and resource protection at the Davidson Seamount, California, U.S.A. In: Cold-water Corals and Ecosystems (eds. Freiwald AR, Roberts JM), pp. 1189-1198. Springer-Verlag, Berlin Heidelberg.

Emerson KJ, Merz CR, Catchen JM, et al. (2010) Resolving postglacial phylogeography using highthroughput sequencing. Proceedings of the National Academy of Sciences of the United States of America 107, 16196-16200.

Glover KA, Skaala O, Limborg M, Kvamme C, Torstensen E (2011) Microsatellite DNA reveals population genetic differentiation among sprat (Sprattus sprattus) sampled throughout the Northeast Atlantic, including Norwegian fjords. Ices Journal of Marine Science 68, 2145-2151.

Gnerre S, MacCallum I, Przybylski D, et al. (2011) High-quality draft assemblies of mammalian genomes from massively parallel sequence data. Proceedings of the National Academy of Sciences of the United States of America 108, 1513-1518.

Harnstrom K, Ellegaard M, Andersen TJ, Godhe A (2011) Hundred years of genetic structure in a sediment revived diatom population. Proceedings of the National Academy of Sciences of the United States of America 108, 4252-4257.

Herrera S, Reyes-Herrera PH, Shank TM (Chapter 2) Genome-wide predictability of restriction sites across the eukaryotic tree of life.

Herrera S, Shank TM (Chapter 5) RAD sequencing enables unprecedented phylogenetic resolution and objective species delimitation in recalcitrant divergent taxa.

Hoegh-Guldberg O, Bruno JF (2010) The impact of climate change on the world's marine ecosystems. Science 328, 1523-1528.

Hohenlohe PA, Bassham S, Etter PD, et al. (2010) Population genomics of parallel adaptation in threespine stickleback using sequenced RAD tags. PLoS Genetics 6, e1000862.

Johnstone IM (2001) On the distribution of the largest eigenvalue in principal components analysis. Annals of Statistics 29, 295-327.

Jones FC, Grabherr MG, Chan YF, et al. (2012) The genomic basis of adaptive evolution in threespine sticklebacks. Nature 484, 55-61.

Lewontin RC, Krakauer J (1973) Distribution of gene frequency as a test of the theory of the selective neutrality of polymorphisms. Genetics 74, 175-195.

Luikart G, Allendorf FW, Cornuet JM, Sherwin WB (1998) Distortion of allele frequency distributions provides a test for recent population bottlenecks. Journal of Heredity 89, 238-247.

Miller MR, Brunelli JP, Wheeler PA, et al. (2012) A conserved haplotype controls parallel adaptation in geographically distant salmonid populations. Molecular Ecology 21, 237-249.

Nedashkovskaya OI, Bum Kim S, Lysenko AM, et al. (2005) Bizionia paragorgiae gen. nov., sp. nov., a novel member of the family Flavobacteriaceae isolated from the soft coral Paragorgia arborea. International Journal of Systematic and Evolutionary Microbiology 55, 375-378.

Patterson N, Price AL, Reich D (2006) Population structure and eigenanalysis. PLoS Genetics 2, e190.

Perrin C, Wing SR, Roy MS (2004) Effects of hydrographic barriers on population genetic structure of the sea star Coscinasterias muricata (Echinodermata, Asteroidea) in the New Zealand fjords. Molecular Ecology 13, 2183-2195.

Prada C, Hellberg ME (2013) Long prereproductive selection and divergence by depth in a Caribbean candelabrum coral. Proceedings of the National Academy of Sciences of the United States of America 110, 3961-3966. 
Price AL, Patterson NJ, Plenge RM, et al. (2006) Principal components analysis corrects for stratification in genome-wide association studies. Nature Genetics 38, 904-909.

Reitzel AM, Herrera S, Layden MJ, Martindale MQ, Shank TM (2013) Going where traditional markers have not gone before: utility of and promise for RAD sequencing in marine invertebrate phylogeography and population genomics. Molecular Ecology 22, 2953-2970.

Roberts JM, Wheeler A, Freiwald AR, Cairns SD (2009) Cold-Water Corals : The Biology and Geology of Deep-Sea Coral Habitats Cambridge University Press, Cambridge, UK ; New York.

Sánchez JA (2005) Systematics of the bubblegum corals (Paragorgiidae: Octocorallia: Cnidaria) with description of new species from New Zealand and the Eastern Pacific. Zootaxa 1014, 1-72.

Suneetha KB, Salvanes AGV (2001) Population genetic structure of the glacier lanternfish, Benthosema glaciale (Myctophidae) in Norwegian waters. Sarsia 86, 203-212.

Tracy CA, Widom H (1994) Level-spacing distributions and the airy kernel. Communications in Mathematical Physics 159, 151-174.

Wating L, France SC, Pante E, Simpson A (2011) Biology of deep-water octocorals. Advances in Marine Biology 60, 41-122.

Wildes SL, Vollenweider JJ, Nguyen HT, Guyon JR (2011) Genetic variation between outer-coastal and fjord populations of Pacific herring (Clupea pallasii) in the eastern Gulf of Alaska. Fishery Bulletin 109, 382-393. 


\section{CHAPTER 7}

\section{Summary and conclusions}

Deep-sea hydrothermal vents and deep-sea coral ecosystems are some of the most conspicuous biological hotspots in the deep-sea. These ecosystems face increasing threats caused by human activities, such as bottom trawling and deep-sea mining. Knowledge of conservation targets is fundamental for the implementation of efficient conservation strategies that help mitigate these threats. Such knowledge must include well-founded taxonomic inventories that allow us to identify species and ecosystems at risk, as well as an understanding of their relatedness, genetic variance, distribution, connectivity patterns, and adaptation potential. Nonetheless, gaining this knowledge in deep-sea ecosystems is difficult due to the extreme challenges of working in this environment, combined with the paucity of genetic resources for deep-sea organisms. Here I provide fundamental high-priority knowledge in taxonomic, evolutionary, and ecological aspects of deep-sea coral and vent species, by harnessing the power of novel genomic tools and overcoming long-standing methodological barriers.

In Chapter 2, I developed bioinformatic tools that help guide the design of studies aiming to characterize eukaryotic genome diversity using restriction-site associated DNA sequencing. With these tools I performed in silico genome-wide surveys thought the eukaryotic tree of life. I tested the hypothesis that genome composition, in terms of GC content, and mono-, di- and trinucleotide composition, can be used to predict the number of restriction sites for a given combination of restriction enzyme and genome across the eukaryotic tree of life. In most cases the trinucleotide genome composition model was the best predictor of the expected number of restriction sites in a eukaryotic genome, and the GC content and mononucleotide models the worst. I conclude that the predictability of restriction site frequencies in eukaryotic genomes needs to be treated on a case-specific basis, whereby the phylogenetic position of the taxon of interest and the specific recognition sequence of the selected restriction enzyme are the chief foci among the most determinant factors. The knowledge gained in this chapter, and the bioinformatic tools developed, was applied in all other subsequent chapters.

In Chapter 3, I tested global-scale historical biogeographic hypothesis of vent fauna using barnacles as model. I characterized the global genetic diversity of vent barnacles to infer their time and place of origin, mode of dispersal, and diversification throughout the world's vents. The approach was to target a suite of 
multiple loci in samples representing seven out of the eight described genera. I also performed restrictionsite associated DNA sequencing (RAD-seq) on individuals from each species. Phylogenetic inferences indicated that vent barnacles have colonized deep-sea hydrothermal vents at least twice in history. The late Mesozoic/Cenozoic was the time of colonization and radiation of barnacles in vent ecosystems. Further analyses suggested that the western Pacific was the place of origin of the major vent barnacle lineage, followed by circumglobal colonization eastward along the southern hemisphere during the Neogene. The inferred time of origin rejects previous hypotheses of antiquity of vent taxa. The timing and the mode of origin, radiation and dispersal are consistent with the inferences made for other deep-sea taxa, including non-vent species, and are correlated with the occurrence of major geological events and mass extinctions. Thus, I suggest that the geological processes and dispersal mechanisms discussed here can explain current distribution patterns of many other marine taxa and have played an important role shaping deep-sea faunal diversity.

In Chapter 4, I examined genetic diversity patterns in vent barnacles at a regional scale. To test the hypothesis that seamounts behave as isolated island-like systems, where population connectivity is limited and endemicity is promoted, I examined genome-wide RAD-seq data from three hydrothermal vent barnacle species. I compared the genetic diversity and population structuring patterns of barnacle populations from seamount and spreading ridges. Among the study populations I found patterns of population genetic structuring that do not conform to the predictions from the seamount endemicity hypothesis. The patterns of genetic variation among individuals collected from seamount and spreading ridges, separated horizontally by hundreds of kilometers and vertically by hundreds of meters, did not reject the null hypothesis of panmixia within each species. I found that these inferences are largely insensitive to the de novo assembly parameters used to identify loci from sequence reads. In conclusion, I suggest that the seamount endemicity hypothesis warrants further testing using high-resolution genetic markers in other vent organisms with differing life history strategies (e.g. brooders) that may limit their dispersal potential, as well as in non-vent organisms, which are not exposed to evolutionary pressures imposed by the dynamic nature of hydrothermal vent systems.

I then moved on to resolve long-standing questions regarding species definitions and relationships in deep-sea corals. In Chapter 5, I demonstrated the empirical utility of RAD-seq by unambiguously resolving phylogenetic relationships among recalcitrant octocoral taxa with divergences greater than 80 million years. I objectively inferred robust species boundaries in the genus Paragorgia, which contains some of the most important ecosystem engineers in the deep-sea, by testing alternative taxonomy-guided or unguided species delimitation hypotheses using the Bayes factors delimitation method (BFD*) with 
genome-wide SNP data. I presented conclusive evidence rejecting the current morphological species delimitation model for the genus Paragorgia and indicating the presence of cryptic species boundaries associated with environmental variables. I argue that the suitability limits of RAD-seq for phylogenetic inferences in divergent taxa cannot be assessed in terms absolute time, but depend on taxon-specific factors such as mutation rate, generation time and effective population size. Classic morphological taxonomy can greatly benefit from integrative approaches that provide objective tests to species delimitation hypothesis.

Finally, in Chapter 6, I explored the adaptation potential of deep-sea coral species to environmental changes by examining a case of adaptation to shallow water from the deep-sea. Few populations of some deep-sea coral species can be found in shallow $(<45 \mathrm{~m})$ high-latitude fjord environments where they experience significantly different environmental conditions than their deep relatives. Therefore, these shallow-water populations are believed to inhabit the extremes of the species' physiological tolerances and likely have developed adaptations that enable them to colonize these shallow-water environments. I aimed to identify potential genomic regions that have enabled the successful adaptation to shallow-water in the deep-sea octocoral species Paragorgia stephencairnsi. To characterize the genome-wide genetic diversity of populations of $P$. stephencairnsi found in shallow-water populations and compare it to the genetic diversity from deep-water populations, I performed high-resolution genome-wide scans of single nucleotide polymorphisms through RAD-seq. I found patterns of significant population genetic differentiation among the examined populations of $P$. stephencairnsi, which are consistent with the hypothesis that larvae from outer deep populations seeded shallow-water inner fjord populations. Furthermore, I find candidate positive-selection markers shared between parallel comparisons of shallow and deep populations, and thus identify them as likely candidate makers for genomic regions involved in adaptation to the shallow-water fjord environment.

Overall, the results from this thesis constitute critical baseline data with which to assess potential effects of anthropogenic disturbances on deep-sea ecosystems. The species delimitation frameworks here developed will enable rapid species assignments as deep-sea specimens from newly explored geographical regions become available. This thesis lays groundwork for describing the impacts of natural selection on deep-sea coral species in the face of environmental changes. The software here developed, and the resulting databases constitute a valuable reference resource that will help guide the choice of restriction enzyme for any study using RAD-seq or related methods. 
I anticipate that the use of novel genomic tools to study deep-sea organisms will accelerate the pace of knowledge acquisition, and thus greatly enhance our understanding of deep-sea ecosystems, their evolution, and their role in the global ecosystems network. However, the speed of genomic data generation has now outpaced the development of analytical tools, and thus there is a great need of developing novel ways to make full use of the information contained in large genomic datasets. 'Omics' techniques promise a fast and direct route to move from descriptive studies in deep-sea organisms to process-oriented studies, which will allow use to understand the mechanisms that have allowed life to thrive in this extreme environment. Understanding these mechanisms can also lead to the development of a myriad of applications that can directly improve human's lives. 